\title{
Consuming Your Feelings: The Mechanisms Underpinning Emotional Eating
}

Pink, Aimee E.

How to cite:

Pink, Aimee E. (2019) Consuming Your Feelings: The Mechanisms Underpinning Emotional Eating. Doctoral thesis, Swansea University.

http://cronfa.swan.ac.uk/Record/cronfa50331

Use policy:

This item is brought to you by Swansea University. Any person downloading material is agreeing to abide by the terms of the repository licence: copies of full text items may be used or reproduced in any format or medium, without prior permission for personal research or study, educational or non-commercial purposes only. The copyright for any work remains with the original author unless otherwise specified. The full-text must not be sold in any format or medium without the formal permission of the copyright holder. Permission for multiple reproductions should be obtained from the original author.

Authors are personally responsible for adhering to copyright and publisher restrictions when uploading content to the repository.

Please link to the metadata record in the Swansea University repository, Cronfa (link given in the citation reference above.)

http://www.swansea.ac.uk/library/researchsupport/ris-support/ 


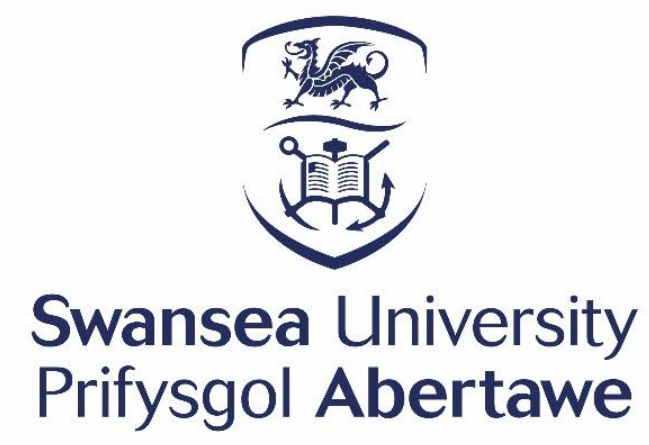

\section{Consuming Your Feelings:}

The Mechanisms Underpinning Emotional Eating

\section{Aimee Elizabeth Pink}

MSc, BSc (Hons)

Submitted to Swansea University in fulfilment of the requirements for the Degree of Doctor of Philosophy 


\section{Declaration}

This work has not previously been accepted in substance for any degree and is not being concurrently submitted in candidature for any degree.

Signed (candidate)

Date.

\section{STATEMENT 1}

This thesis is the result of my own investigations, except where otherwise stated. Where correction services have been used, the extent and nature of the correction is clearly marked in footnote(s).

Signed (candidate)

Date.

\section{STATEMENT 2}

I hereby give consent for my thesis, if accepted, to be available for photocopying and for inter-library loan, and for the title and summary to be made available to outside organisations.

Signed (candidate)

Date. 


\section{Acknowledgements}

Firstly, a big thank you to my two supervisors, Dr Claire Williams and Professor Michelle Lee, who have made this journey a little easier with their belief in me and support throughout. It has been a huge help in being able to call upon their expertise and knowledge. Their guidance has been invaluable across all aspects and I cannot thank them enough.

Thank you to all the participants who have taken part in my research and to Amy Davies and Sophie Harrison for their assistance in recruitment and data collection of Study Five.

Additional thanks to Ian Russell, Gary Freeman, Neil Carter and Carlo Lucignoli for their fantastic technical support and their patience with my poor technical abilities. I would also like to thank my fellow $\mathrm{PhD}$ students at Swansea university and particularly, the office of 809; Laura, Alecia, Rhian, Amy, Emma, Holly, Chloe, Nasreem, Xiaosheng, Simon, and Joel. They have all helped, inspired and motivated me to the finish and it was a privilege to have shared the $\mathrm{PhD}$ experience with them. A special mention to Dr Menna Price, like an older PhD sister to me, who aided me with top tips and advice throughout the last 4 years. Also, to Dr Hayley Young, for helping me in the world of interoceptive awareness.

A thank you must go to Swansea University Ladies and Mens Hockey Club for ensuring I still managed a competitive edge during my $\mathrm{PhD}$ and providing me with a fabulous set of team mates for support and memories to accompany. My friends and family have been a huge support and I cannot thank them enough for helping me get to this point. I look forward to the cluedo jokes! My parents have encouraged me through 8 years at university and I hope they are proud. To my brother, Callum, always there when I need him, and I wish him luck on his own PhD journey now. And finally, a massive thank you to Harry, for being my rock. He has shared the emotional rollercoaster ride with me that is completing a $\mathrm{PhD}$ and has been fully supportive the entire way. 


\begin{abstract}
Obesity continues to have a huge impact on society, with emotional eating considered to be one of many driving factors. Consequently, the overarching aim of this thesis is to investigate the mechanisms underpinning emotional eating, paying specific attention to the role of emotional dysregulation. Emotional dysregulation involves a combination of emotional vulnerability and an inability to regulate emotional responses which can lead to the adoption of maladaptive coping strategies, including emotional eating. However, emotional dysregulation also encompasses many of the features characteristic of alexithymia, including difficulty identifying and describing feelings. As a result, and in order to further elucidate the complex mechanisms underpinning emotional eating, alexithymia is used as a correlate of emotional dysregulation in this thesis. In addition, as alexithymia has been related to both impulsiveness and negative affect, and that emotional eating is grounded in the idea that the consumption of food follows an emotional experience, negative urgency and negative affect will also be explored as key constructs.
\end{abstract}

Chapter One reviews the current literature on emotional eating, alexithymia, impulsivity and negative affect. It also sets out the aims and objectives of this thesis, putting forward a novel theoretical model to examine the influence of emotional dysregulation on emotional eating, and in turn, body mass index.

Chapter Two presents the results of two studies (an exploratory analysis in a student sample and a self-replication in a more representative general population sample) examining the factors mediating the relationship between emotional dysregulation, emotional eating, and body mass index. In addition to significant associations between emotional dysregulation and emotional eating in the student sample, negative affect and negative urgency were found to mediate the relationship between alexithymia and emotional eating. However, even though significant associations between emotional dysregulation and emotional eating were confirmed in the general population sample, different pathways emerged. Specifically, negative affect was the only significant mediator between alexithymia and emotional eating. 
Chapters Three and Four focus on whether simulating emotional dysregulation in an experimental setting directly affects food intake. Specifically, a novel method of inducing emotional confusion as an analogue of alexithymia is developed and piloted in Chapter Three, followed by a fully powered mood manipulation study in Chapter Four to assess food intake following the induction of positive, negative and emotionally confusing mood states. Compared to a control condition, food intake did not differ significantly across experimental mood conditions.

Finally, in Chapter Five, the initial theoretical model described at the end of Chapter One is extended to include interoceptive awareness and feeling fat. Here, the ability to trust bodily sensations and the experience of feeling fat was found to significantly mediate the relationship between emotional dysregulation and emotional eating. The model is also extended to include negative urgency, with increased levels of alexithymia related to a reduction in trusting bodily sensations, which in turn increases the tendency to experience sensations of feeling fat, followed by reacting rashly to alleviate the associated feelings (negative urgency), and therefore, an increased propensity to engage in emotional eating.

Overall, the research presented in this thesis takes the first steps in developing an inclusive theoretical model of emotional eating. Whilst the precise nature in which emotional dysregulation influences emotional eating remains unclear, the results of this thesis nevertheless supports the role of emotional dysregulation and has important implications for the development of interventions to aid weight loss/management. 


\section{Dissemination of Research}

\section{Publications}

Pink, A., Williams, C., \& Lee, M. (2016). Exploring the relationships between alexithymia, impulsivity, emotional eating and BMI. Appetite, 107, 689. [Conference proceedings] - Study One

Pink, A, Williams, C, \& Lee, M. (2018). The induction of emotionally confusing feelings and the impact on food intake. Appetite, 123, 457. [Conference proceedings] - Study Four

Pink, A., Price, M., Lee, M., Young, H., \& Williams, C. (2018). Why do I feel so fat? The role of poor emotional and physical sensation processing in feeling fat. Appetite, 130, 311-312. [Conference proceedings] - Study Five

Pink, A. E., Lee, M., Price, M., \& Williams, C. (2019). A serial mediation model of the relationship between alexithymia and BMI: The role of negative affect, negative urgency and emotional eating. Appetite, 133, 270-278. - Study One and Two

\section{Poster and Oral Presentations}

2016 British Feeding and Drinking Group - Oral: Exploring the relationship between Alexithymia, Impulsivity, Emotional Eating and BMI - Study One

2016 Swansea University 3 Minute Thesis - Oral: Emotional Eating: What makes us vulnerable?

2017 British Feeding and Drinking Group - Oral: The induction of emotionally confusing feelings and the impact on food intake - Study Four

2017 Society of the Study of Ingestive Behaviour - Poster: The effect of manipulated mood on food intake - Study Four 
2017 Swansea University 3 Minute Thesis - Oral: Can Manipulating Mood Influence Snack Intake?

2017 Contemporary and Future Research into Alexithymia - Poster: Exploring the relationship between alexithymia, impulsivity and emotional eating - Study One

2017 Contemporary and Future Research into Alexithymia - Poster: The development of a novel mood manipulation task to induce emotional confusion - an analogue of alexithymia - Study Three

2017 Contemporary and Future Research into Alexithymia - Poster: The emotional expression of affect within a novel mood induction paradigm - Study Three

2017 Contemporary and Future Research into Alexithymia - Poster: The effect of manipulated mood on food intake - Study Four

2018 British Feeding and Drinking Group - Oral: Why do I feel so fat? The role of poor emotional and physical sensation processing - Study Five

\section{Prizes and Awards}

2016 Best Oral Presentation Swansea University Human and Health Sciences Conference

2017 Runner-up Poster Presentation - Experimental Psychology Society Alexithymia Workshop

2017 Runner-up Oral Presentation - Swansea University Human and Health Sciences Conference 


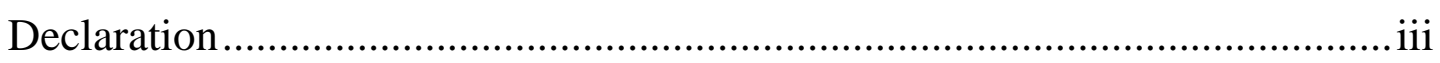

Acknowledgements................................................................................ iv

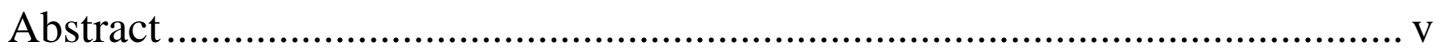

Dissemination of Research ..................................................................... vii

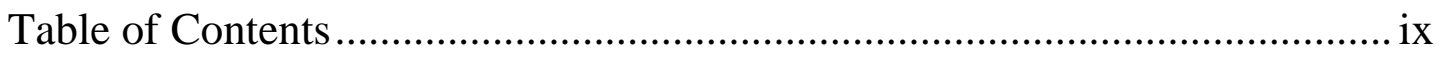

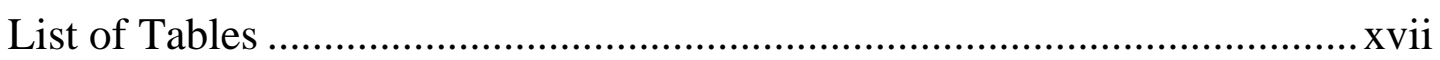

List of Figures ……………………...................................................

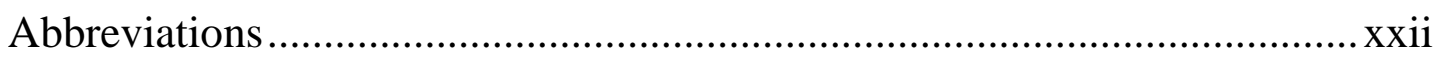

Chapter One: Introduction and Literature Review ……………………….... 25

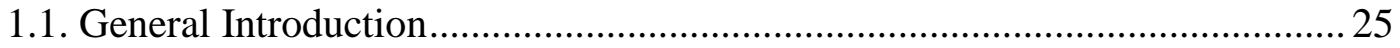

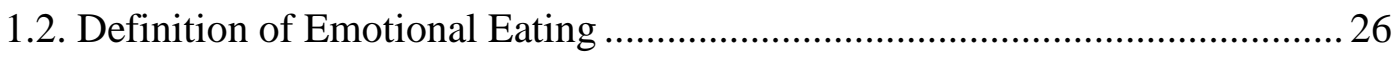

1.3. Emotional Eating and Body Mass Index ........................................................ 29

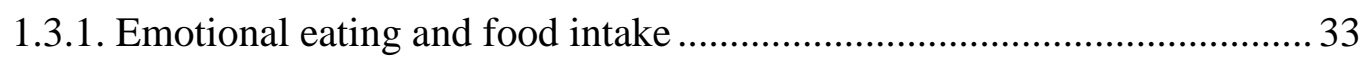

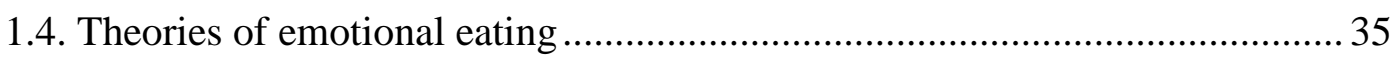

1.5. Emotional dysregulation............................................................................ 40

1.5.1. Emotional dysregulation and eating behaviours ......................................... 40

1.5.2. Emotional Dysregulation and Body Mass Index ....................................... 42

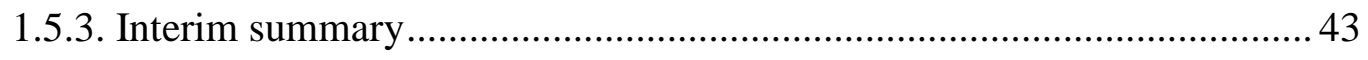

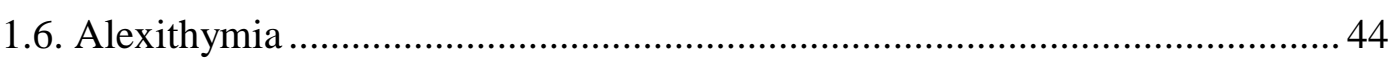

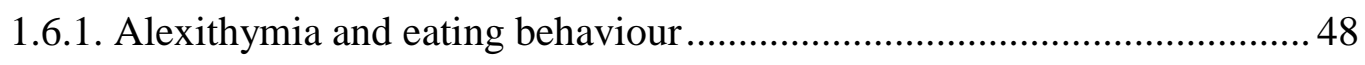

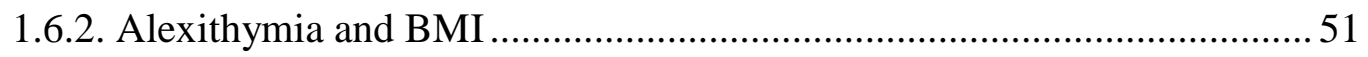

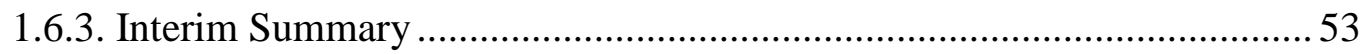

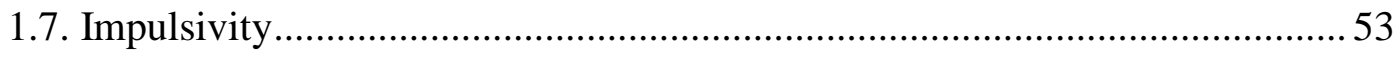

1.7.1. Impulsivity within eating behaviours .................................................... 55

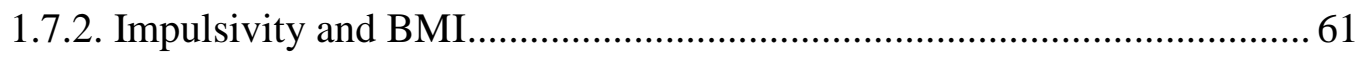

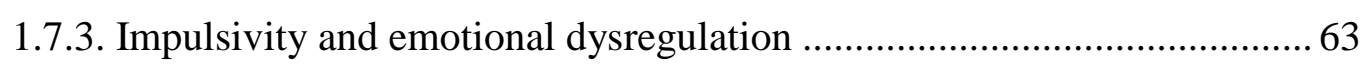

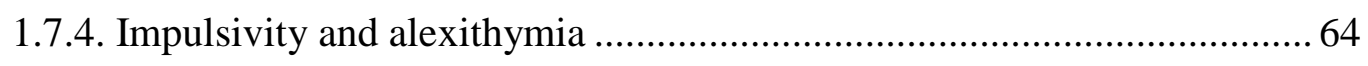

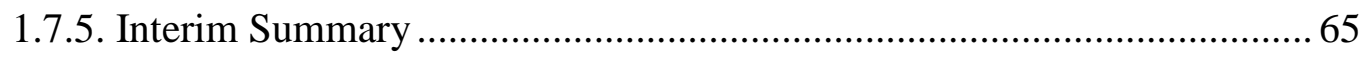

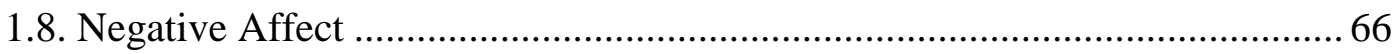

1.8.1. Negative affect in eating behaviours ......................................................... 67 


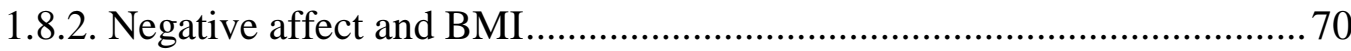

1.8.3. Negative affect and emotional dysregulation ....................................... 72

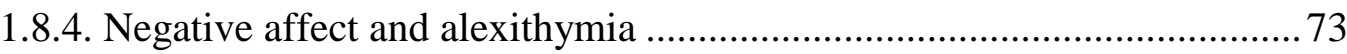

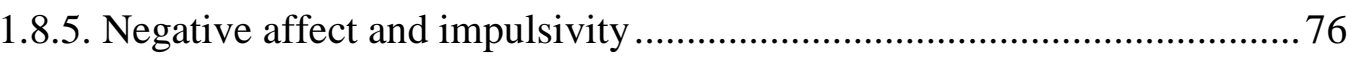

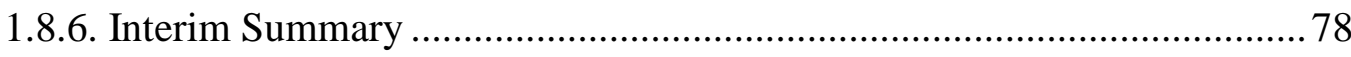

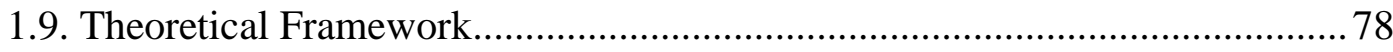

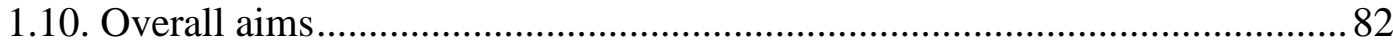

Chapter Two: The relationship between Alexithymia, Negative Affect, Impulsivity, Emotional Eating and BMI................................................ 84

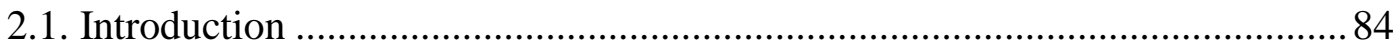

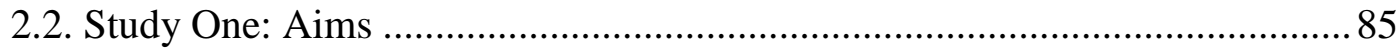

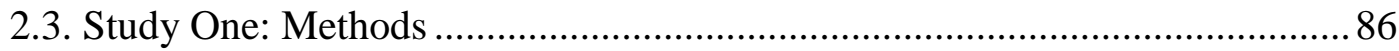

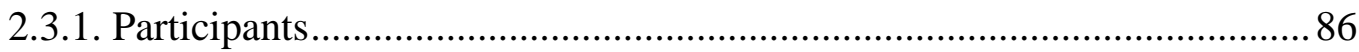

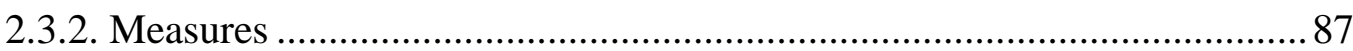

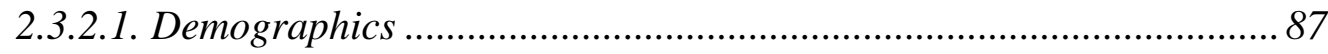

2.3.2.2. Positive Affect and Negative Affect Schedule ...................................8 87

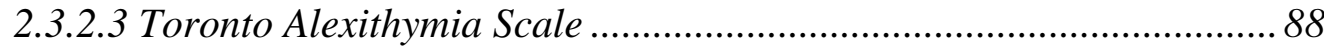

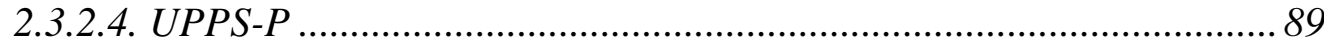

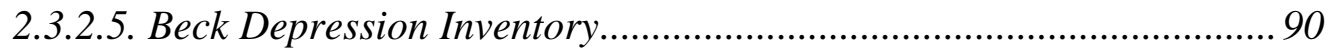

2.3.2.6. Beck Anxiety Inventory ....................................................................... 90

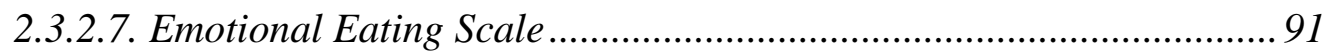

2.3.2.8. Three Factor Eating Questionnaire - Revised ............................... 91

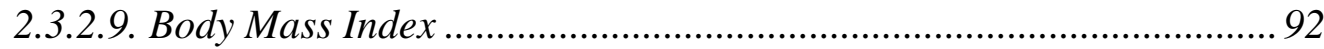

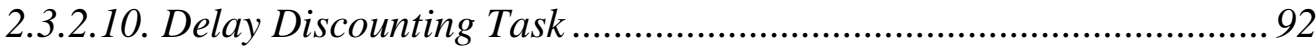

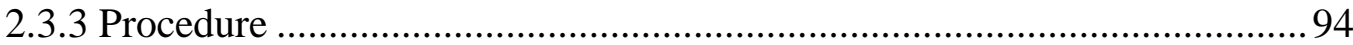

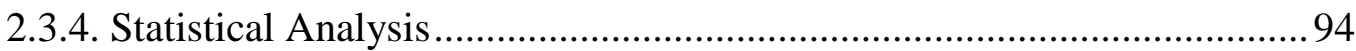

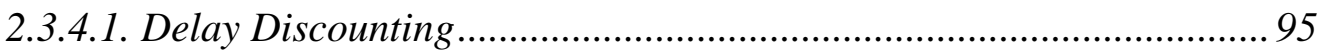

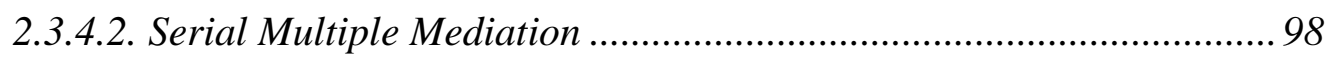

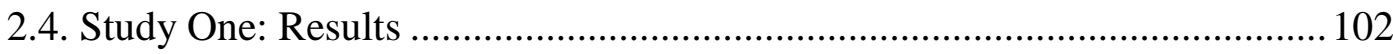

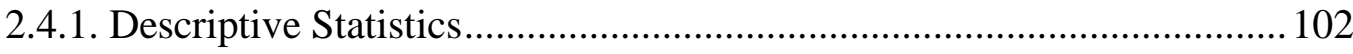

2.4.1.1. Delay Discounting ............................................................................ 103

2.4.2. Correlational Analysis ...................................................................... 105 
2.4.2.1. Correlations between alexithymia, negative affect, negative urgency, emotional eating and BMI.

2.4.2.2. Correlations between delay discounting, alexithymia, negative urgency, emotional eating and BMI....................................................... 107

2.4.3. Serial Multiple Mediation for Emotional Eating ................................... 107

2.4.4. Exploratory analysis of serial multiple mediation for BMI................... 111

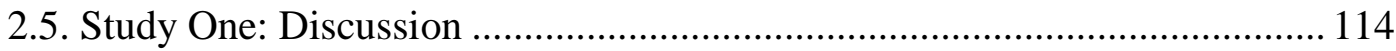

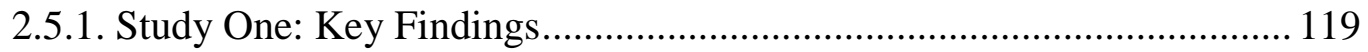

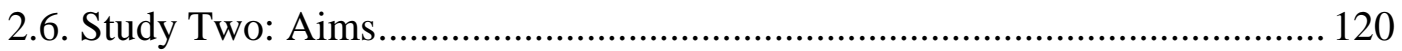

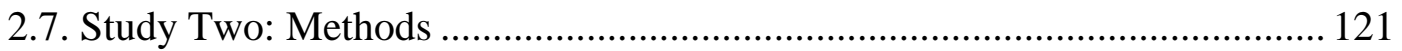

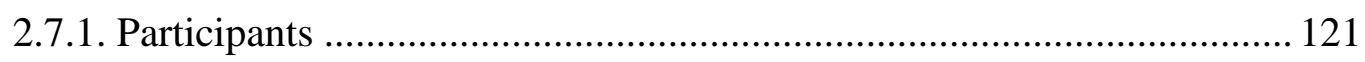

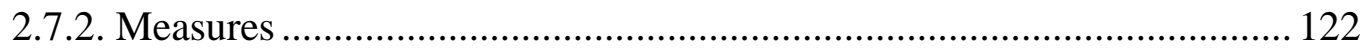

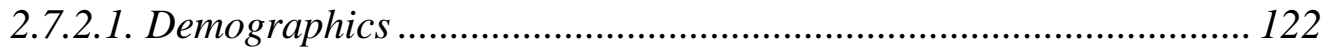

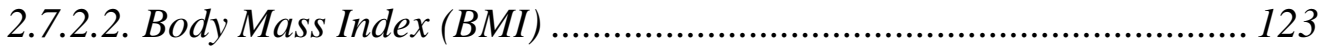

2.7.2.3. Positive Affect and Negative Affect Scale .................................... 123

2.7.2.4. Toronto Alexithymia Scale ................................................................. 123

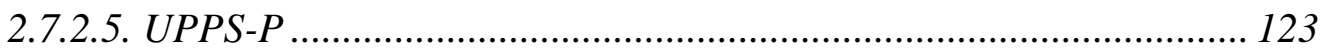

2.7.2.6. Beck Depression Inventory ............................................................ 123

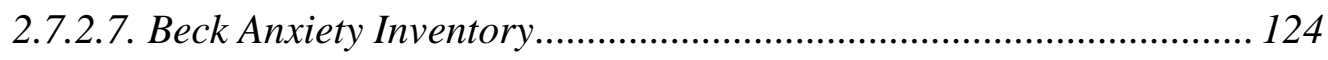

2.7.2.8. Emotional Eating Scale ................................................................. 124

2.7.2.9. Three Factor Eating Questionnaire - Revised............................... 124

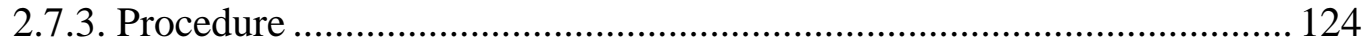

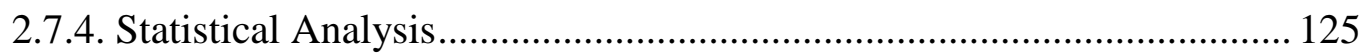

2.7.4.1. Serial Mediation Model................................................................ 125

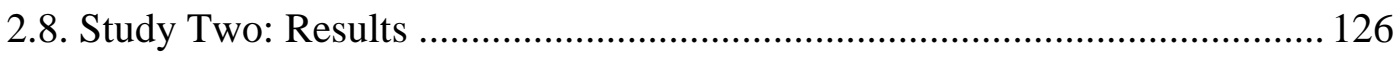

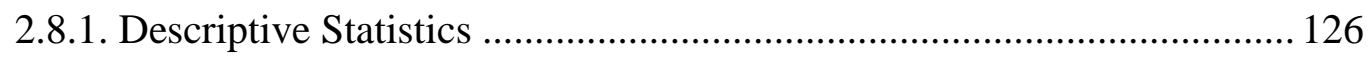

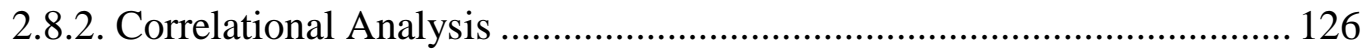

2.8.2.1. Correlations between alexithymia, negative urgency, negative affect, emotional eating and BMI.......................................................................... 126

2.8.3. Serial multiple mediation models for emotional eating ......................... 129

2.8.4. Serial multiple mediation Models for BMI ......................................... 130

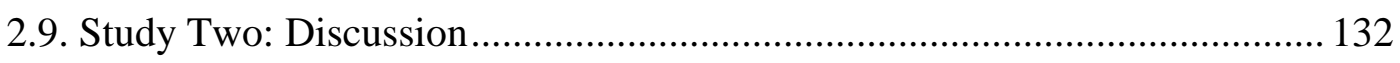

2.9.1. Study Two: Key Findings …............................................................. 134

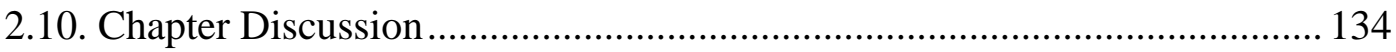




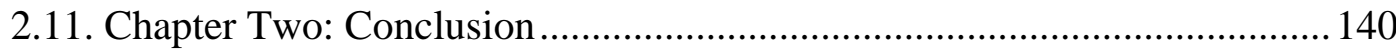

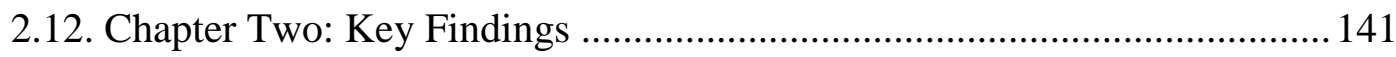

Chapter Three: Development of a novel mood manipulation paradigm ....... 142

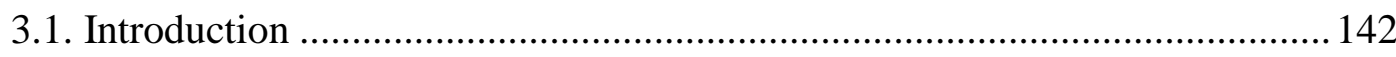

3.2. Literature Review of Mood Induction Procedures …................................... 143

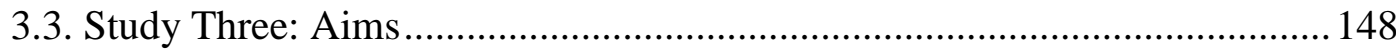

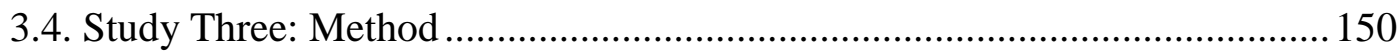

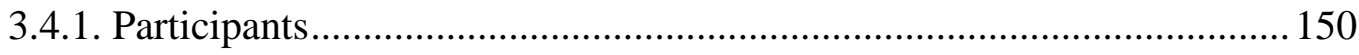

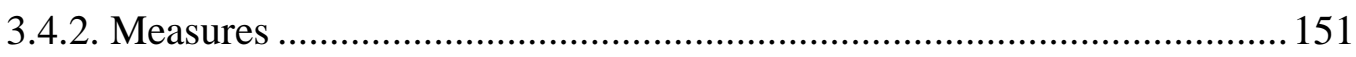

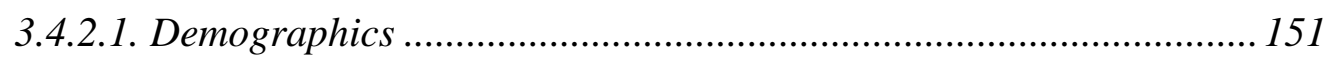

3.4.2.2. Profile of Mood States.................................................................. 151

3.4.2.3. Subjective measure of Mixed Emotion ............................................ 152

3.4.2.4. Toronto Alexithymia Scale ........................................................... 152

3.4.2.5. Beck Depression Inventory-II....................................................... 152

3.4.2.6. Beck Anxiety Inventory .............................................................. 153

3.4.2.7. Memory Characteristics Questionnaire ........................................ 153

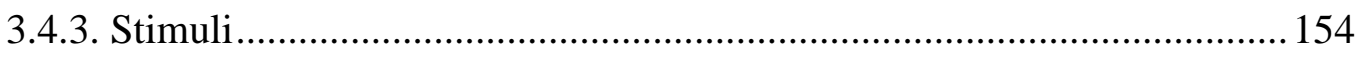

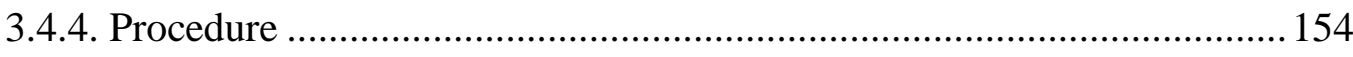

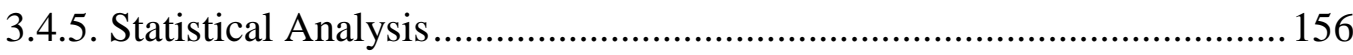

3.4.5.1. Quantitative Statistics ................................................................. 156

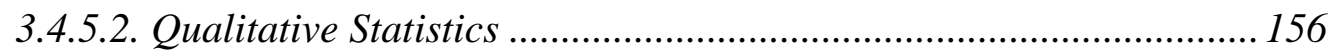

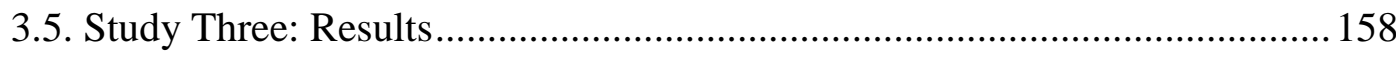

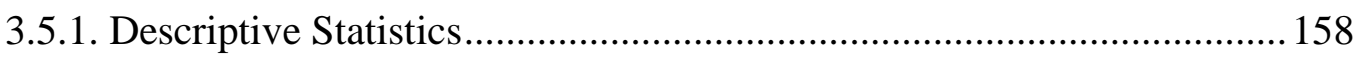

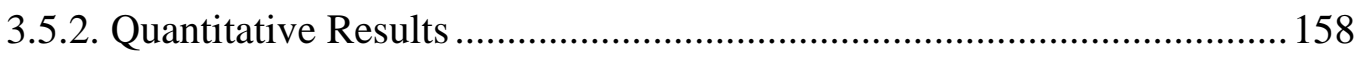

3.5.2.1. Manipulation Check: Comparison of POMS and SME scores pre-and

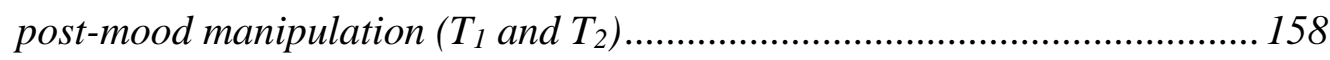

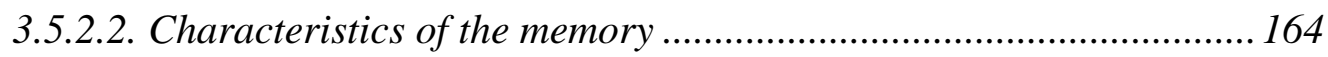

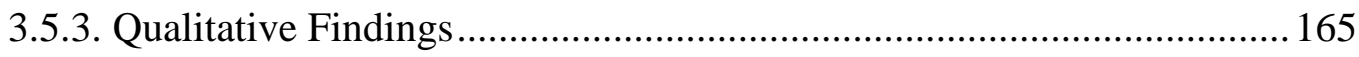

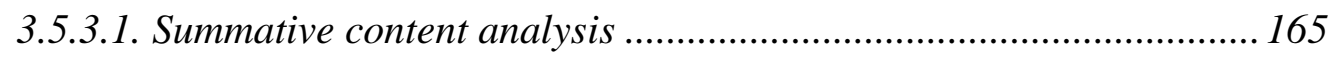

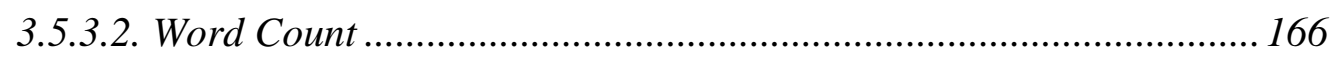

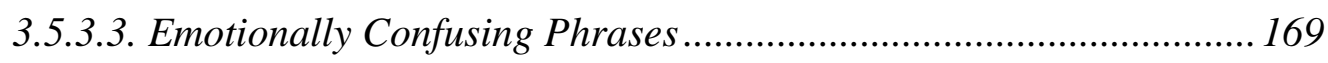

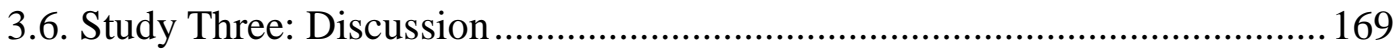

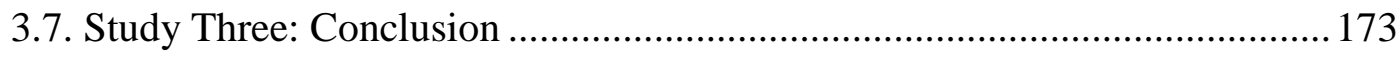

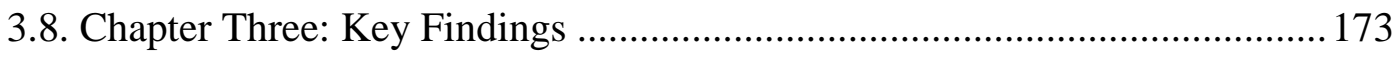


Chapter Four: The Effects of Mood Manipulation on Food Intake 175

4.1. Introduction 175

4.2. Mood Induction in Eating Behaviours 175

4.3. Bogus Taste Test 182

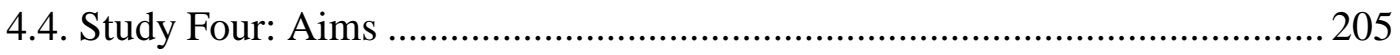

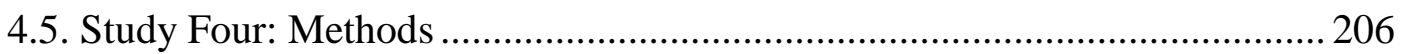

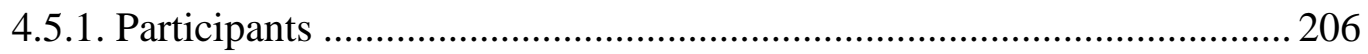

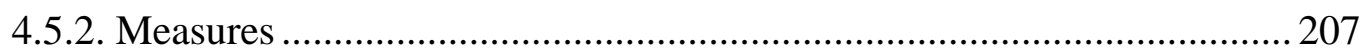

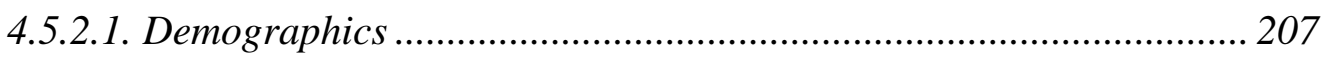

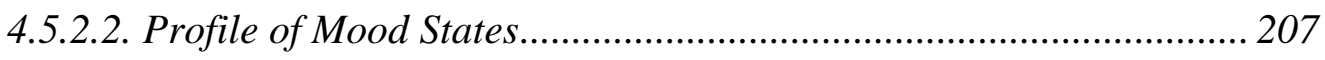

4.5.2.3. Subjective measure of Mixed Emotions.......................................... 207

4.5.2.4. Toronto Alexithymia Scale ............................................................. 208

4.5.2.5. Beck Depression Inventory-II ...................................................... 208

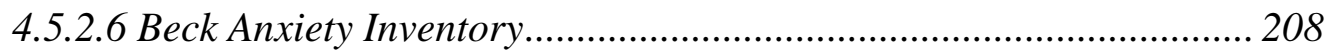

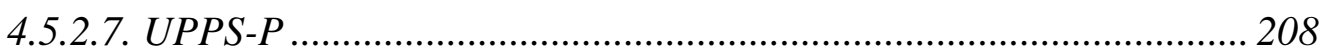

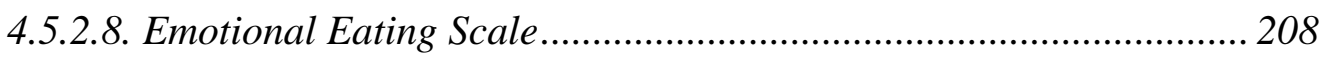

4.5.2.9. Three Factor Eating Questioned - revised ................................... 208

4.5.2.10. Memory Characteristic Questionnaire ......................................... 209

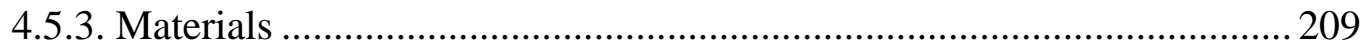

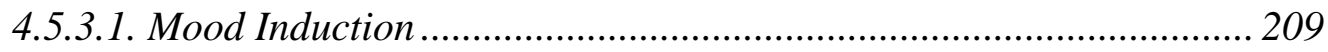

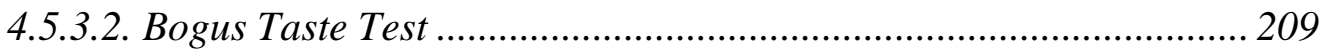

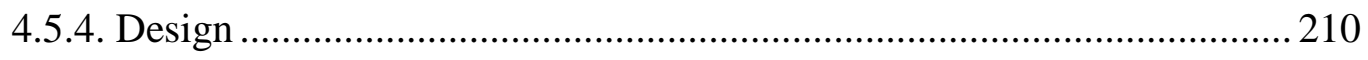

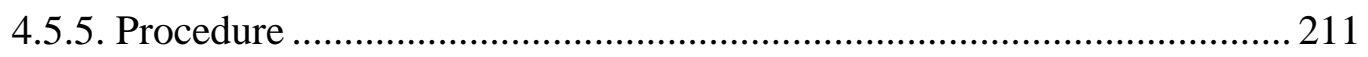

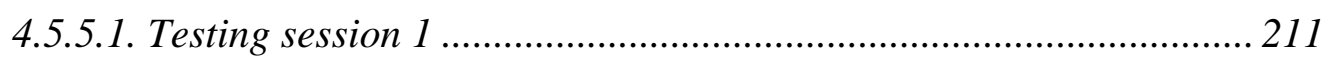

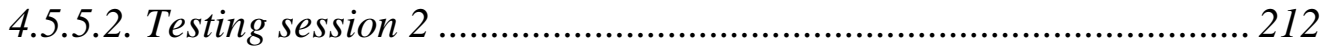

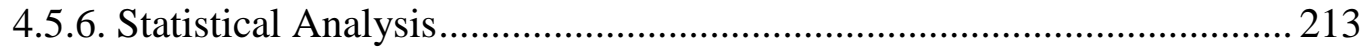

4.5.6.1. Manipulation check ....................................................................... 213

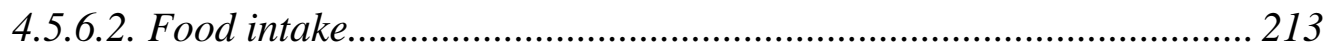

4.5.6.3. Serial mediation model ................................................................... 214

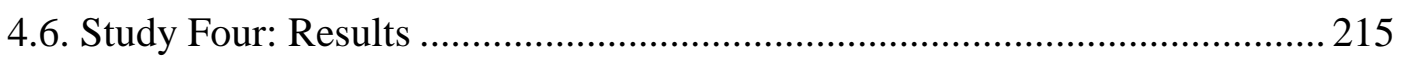

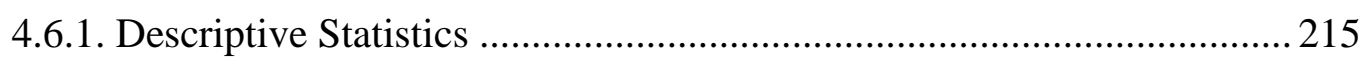

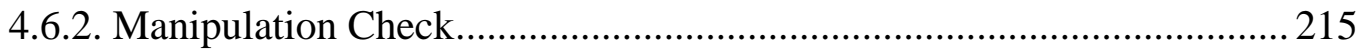




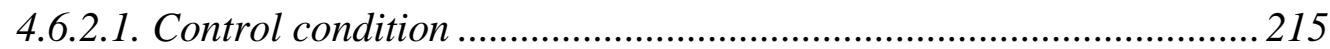

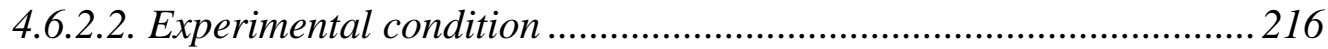

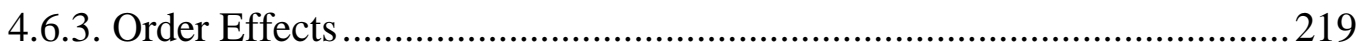

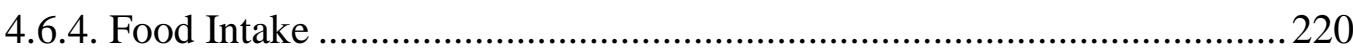

4.6.4.1. Food intake differences across levels of alexithymia ...................... 221

4.6.4.2. Food intake across high and low emotional eating ........................ 221

4.6.5. Serial Mediation Model .................................................................... 222

4.6.5.1. Alexithymia predicting food intake ............................................... 222

4.6.5.2. Alexithymia predicting emotional eating ........................................ 222

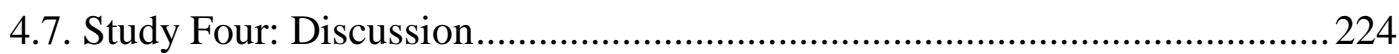

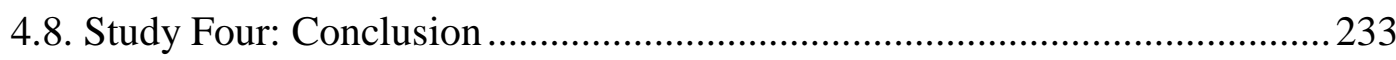

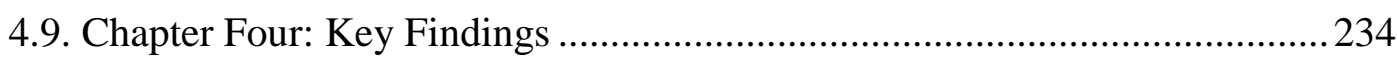

Chapter Five: The relationship between Alexithymia, Emotional Eating, Interoceptive Awareness, and Feeling Fat ............................................2236

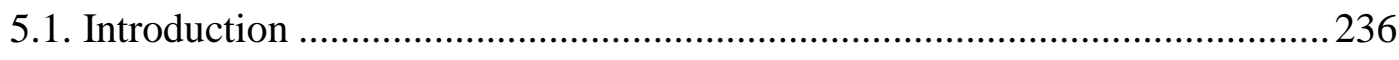

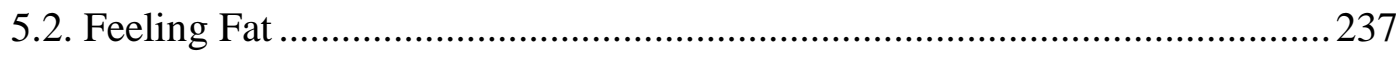

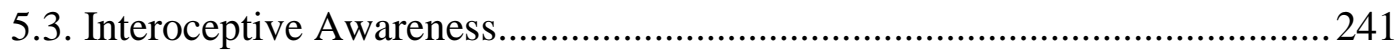

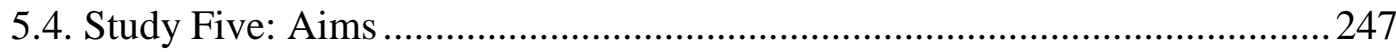

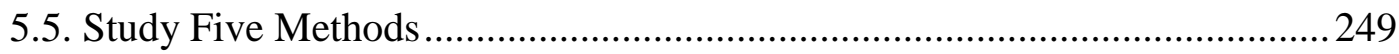

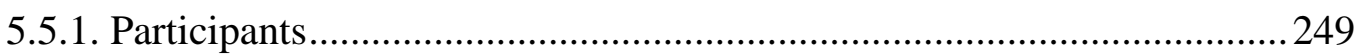

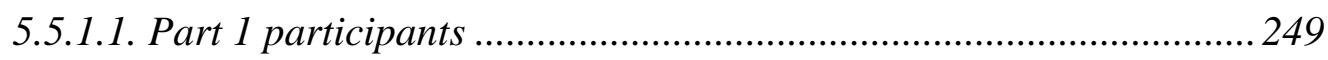

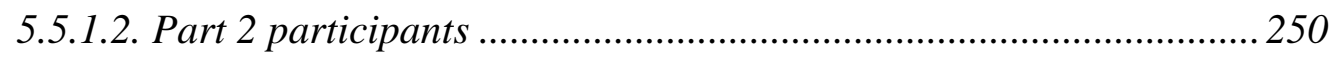

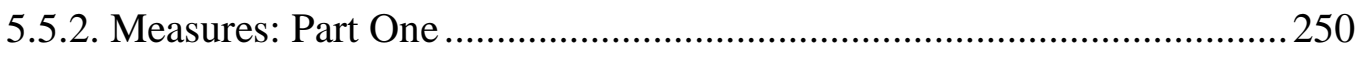

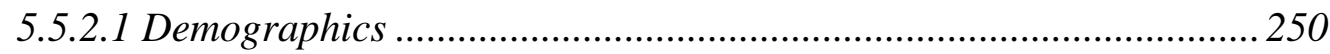

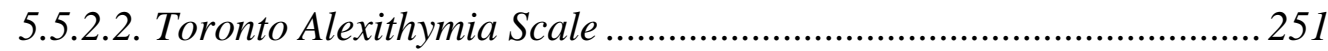

5.5.2.3. Body Attitudes Questionnaire ......................................................... 251

5.5.2.4. Multidimensional Assessment of Interoceptive Awareness.............. 251

5.5.2.5. Dutch Eating Behaviour Questionnaire .......................................... 253

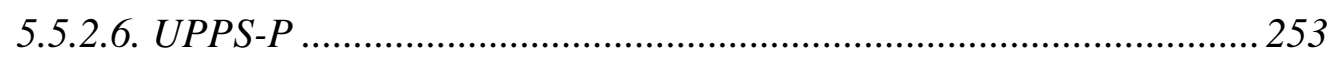

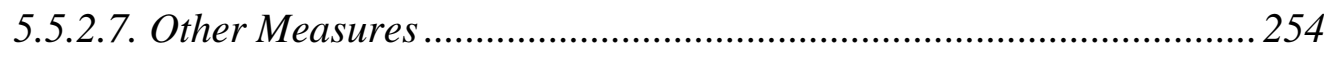

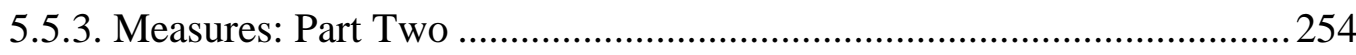




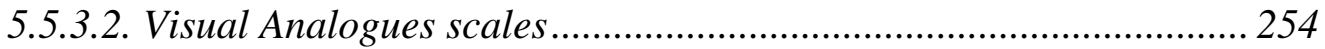

5.5.3.3. Heartbeat Detection Task ............................................................... 255

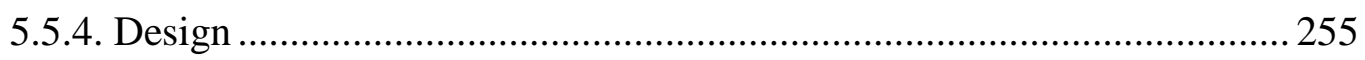

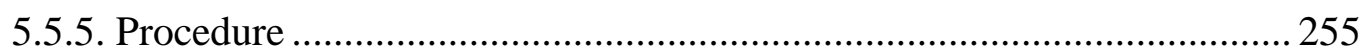

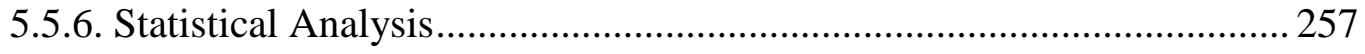

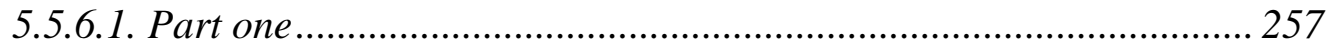

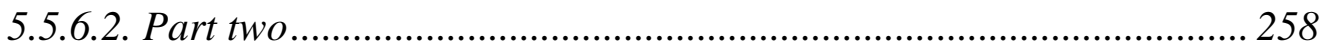

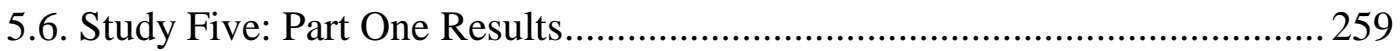

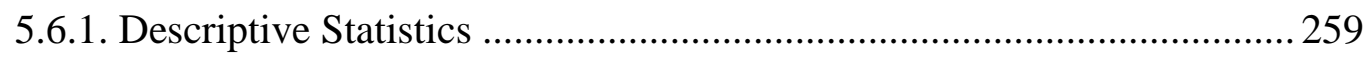

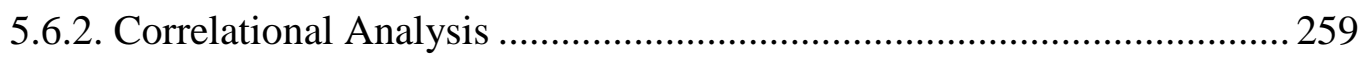

5.6.2.1. Correlations between emotional eating, feeling fat, alexithymia and

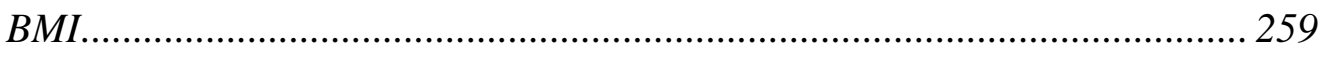

5.6.2.2. Correlations between emotional eating, feeling fat, interoceptive

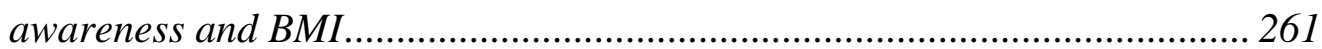

5.6.2.3. Correlations between alexithymia and interoceptive awareness.... 261

5.6.2.4. Correlations between interoceptive awareness, feeling fat and negative

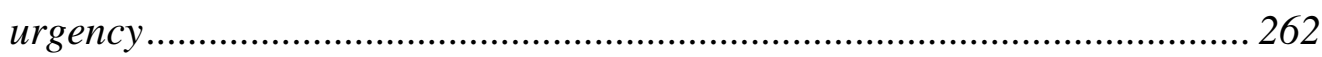

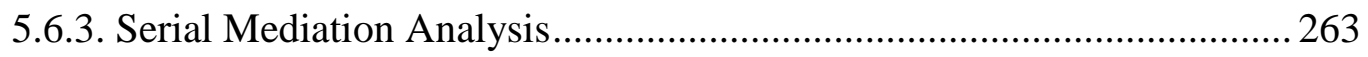

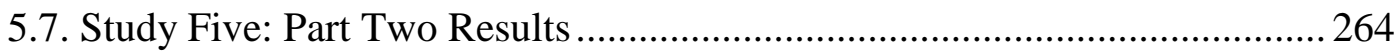

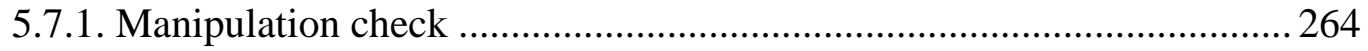

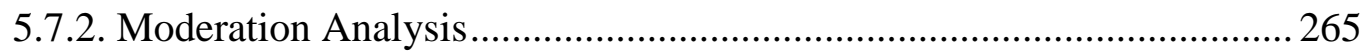

5.8. Study Five: Final Model ............................................................................... 269

5.8.1. The relationship between alexithymia and emotional eating, including interoception, negative urgency and feeling fat ............................................ 269

5.8.2. The relationship between alexithymia and emotional eating including interoception, negative urgency and feeling fat ............................................ 272

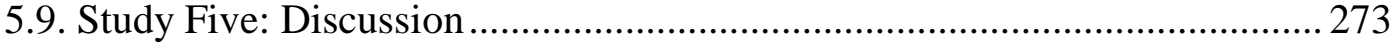

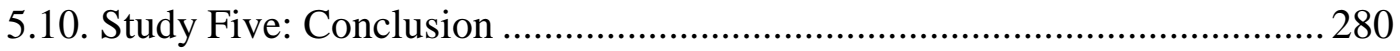

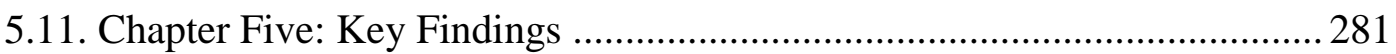

Chapter Six: Discussion and Conclusion .............................................. 282

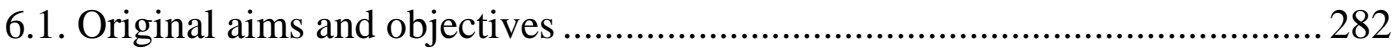

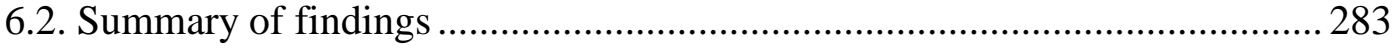

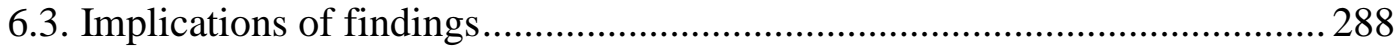




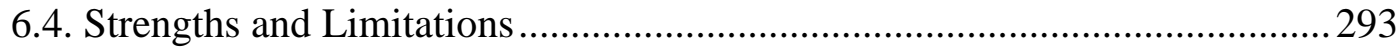

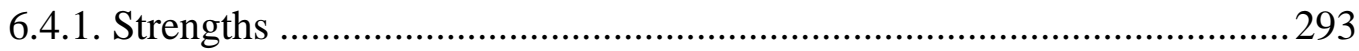

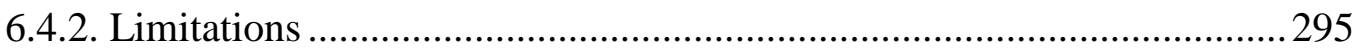

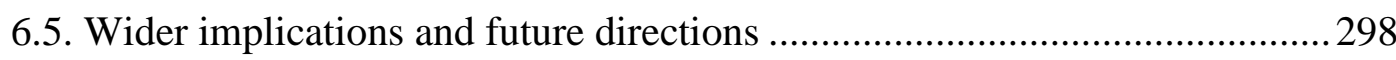

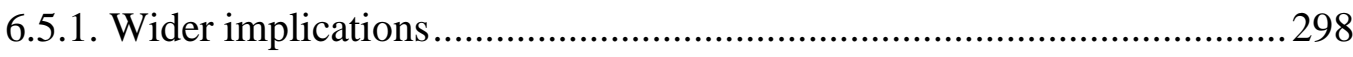

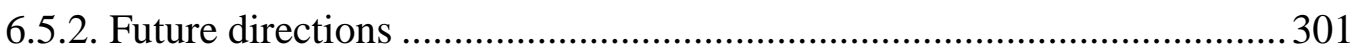

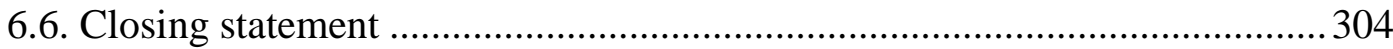

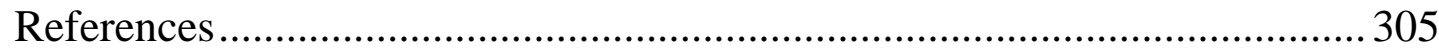

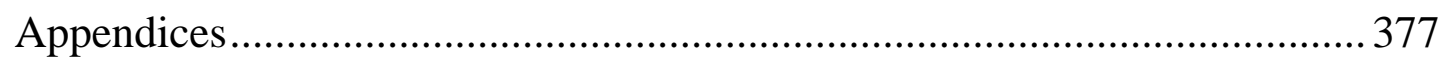




\section{List of Tables}

Table 1. The serial multivariate mediation pathways analysed in Study One.

Table 2. Mean, standard deviation and range for each study variable in Study One.

Table 3. Mean, standard deviation and range for each delay discounting parameter.

Table 4. Pearson correlations between alexithymia, negative urgency, negative affect, emotional eating and BMI for Study One.

Table 5. Correlations between delay discounting parameters and BMI, emotional eating, alexithymia and negative urgency.

Table 6. Reasons for participant exclusions in Study Two.

Table 7. Mean, standard deviation and range for each study variable in Study Two.

Table 8. Pearson correlations between alexithymia, negative urgency, negative affect, emotional eating and BMI for Study Two.

Table 9. Total and group mean, standard deviation and range for alexithymia, depression and anxiety for Study Three.

Table 10. Baseline $\left(T_{1}\right)$ and post-manipulation $\left(T_{2}\right)$ means and standard deviations for the POMS subscales, POMS overall mood and the SME.

Table 11. Themes of autobiographical memories recalled in Study Three.

Table 12. The mean (SD) percentage of affect words in each group.

Table 13. Overview of mood induction and food intake studies.

Table 14. Serial multivariate mediation models analysed in Study Four. 
Table 15. Total and group means, standard deviations and ranges for each study variable in Study Four.

Table 16. Mean (SD) food intake in grams for the control and experimental conditions across positive, negative and emotionally confusing groups.

Table 17. Additional serial multivariate mediation model pathway analysed in Study Four.

Table 18. Reasons for participant exclusions in Study Five.

Table 19. Serial multivariate mediation pathways analysed in Study Five.

Table 20. Mean, standard deviation and range for each study variables in Study Five Part One.

Table 21. Pearson correlations between emotional eating, feeling fat, alexithymia and BMI.

Table 22. Pearson correlations between emotional eating, feeling fat, BMI and the MAIA subscales.

Table 23. Pearson correlations between alexithymia and interoceptive awareness. 


\section{List of Figures}

Figure 1. Inclusive model of emotional eating: The proposed direct and indirect relationships between alexithymia and emotional eating, including negative affect and impulsivity as mediators.

Figure 2. Example situation of a cycle of emotional eating.

Figure 3. Inclusive model of BMI: The proposed direct and indirect relationships between alexithymia and BMI including negative affect, impulsivity and emotional eating as mediators.

Figure 4. Serial mediation model of alexithymia (TAS-20) on emotional eating (TFEQ-EE) including depression (BDI) and negative urgency (NU) as mediators.

Figure 5. Serial mediation of alexithymia (TAS-20) on BMI including depression (BDI), negative urgency (NU) and emotional eating (TFEQ-EE) as mediators.

Figure 6. Example PROCESS serial multiple mediation output to identify the direct and indirect effects between alexithymia (TAS-20) and emotional eating (TFEQ-EE) including depression (BDI) and negative urgency (NU) as mediators.

Figure 7. Example PROCESS serial multiple mediation output to identify the direct and indirect effects between difficulty describing feelings (DDF) and emotional eating (TFEQ-EE) including anxiety (BAI) and negative urgency $(\mathrm{NU})$ as mediators.

Figure 8. Example PROCESS serial multiple mediation output to identify the direct and indirect effects between difficulty describing feelings (DDF) and body 
mass index (BMI) including anxiety (BAI), negative urgency (NU) and emotional eating as mediators.

Figure 9. Overall POMS scores for control, positive, negative and emotional confusing groups.

Figure 10. POMS elatedness subscale scores for control, positive, negative and emotional confusing groups.

Figure 11. SME scores for control, positive, negative and emotional confusing groups.

Figure 12. Differences in total POMS score baseline $\left(T_{1}\right)$ to post-mood $\left(T_{2}\right)$ for the positive, negative and emotional confusion groups.

Figure 13. Difference in food intake between the control and experimental condition for the positive, negative and emotional confusing groups controlling for cognitive restraint, hunger and BMI scores.

Figure 14. Inclusive model of emotional eating: The proposed direct and indirect relationships between alexithymia and emotional eating including interoceptive awareness and feeling fat.

Figure 15. Example PROCESS serial multiple mediation output to identify the direct and indirect effects between difficulty describing feelings (DDF) and body mass index (BMI) including anxiety (BAI), negative urgency (NU) and emotional eating as mediators.

Figure 16. The interactions between total alexithymia and attention regulation scores for differences in feeling fat scores.

Figure 17. The interactions between total alexithymia and emotional awareness scores for differences in feeling fat scores. 
Figure 18. Inclusive model of emotional eating: The proposed direct and indirect relationships between alexithymia and emotional eating including MAIA trusting, feeling fat and negative urgency.

Figure 19. Inclusive model of emotional eating: The proposed direct and indirect relationships between alexithymia (TAS-20) and emotional eating (DEBQ) including negative affect (NA), MAIA trusting (T), feeling fat (FF) and negative urgency (NU) as mediators.

Figure 20. Figure 1 as presented in Chapter One. Inclusive model of emotional eating: The proposed direct and indirect relationships between alexithymia and emotional eating, including negative affect and impulsivity as mediators. 


\section{Abbreviations}

AN

ANG/FRUS

ANX

AUC

BAI

BAQ

BDI-II

BED

BN

BMI

CI

DD

DEBQ

DEBQ-EE

DEBQ-XE

DEBQ-R

DEP

DIF

DDF

EDI-2

EOT

EES

FF

HRHD

HRLD

IP

$\boldsymbol{k}$

LLR

LRHD

LRLD

MAIA
Anorexia Nervosa

Anger/Frustration (EES Subscale)

Anxiety (EES Subscale)

Area under the Curve

Beck's Anxiety Inventory

Body Attitudes Questionnaire

Beck's Depression Inventory

Binge Eating Disorder

Bulimia Nervosa

Body Mass Index

Confidence Interval

Delayed Discounting

Dutch Eating Behaviour Questionnaire

Emotional Eating (DEBQ Subscale)

External Eating (DEBQ Subscale)

Restraint Eating (DEBQ Subscale)

Depression (EES Subscale)

Difficult Identifying Feelings

Difficultly Describing Feelings

Eating Disorders Inventory - 2

Externally Oriented Thinking

Emotional Eating Scale

Feeling Fat

High Restraint, High Disinhibition

High Restraint, Low Disinhibition

Indifference Point

Hyperbolic Temporal Discounting Parameter

Larger, longer reward

Low Restraint, High Disinhibition

Low Restraint, Low Disinhibition

Multidimensional Assessment of Interoceptive Awareness 
MAIA-N

MAIA-ND

MAIA-AR

MAIA-EA

MAIA-SR

MAIA-BL

MAIA-T

NA

Neg-FF

Neg-SS

Neut

NU

PA

PANAS

PERS

Pos-FF

PREM

PU

Q

SS

SSR

TAS-20

TFEQ

TFEQ-CR

TFEQ-EE

TFEQ-R18

TFEQ-UE

UPPS

UPPS-P

VAS
Noticing (MAIA Subscale)

Not Distracting (MAIA Subscale)

Awareness Regulation (MAIA Subscale)

Emotional Awareness (MAIA Subscale)

Self-Regulation (MAIA Subscale)

Body Listening (MAIA Subscale)

Trusting (MAIA Subscale)

Negative Affect

Negative Feeling Fat - experimental condition

Negative Social Situation - experimental condition

Neutral - experimental condition

Negative Urgency

Positive Affect

Positive Affect and Negative Affect Scale

Perseveration (UPPS-P Subscale)

Positive Feeling Fat - experimental condition

Premeditation (lack of; UPPS-P Subscale)

Positive Urgency

Reward Utility Parameter

Sensation Seeking (UPPS-P Subscale)

Smaller, sooner reward

Toronto Alexithymia Scale - 20 items

Three Factor Eating Questionnaire

Cognitive Restraint (TFEQ Subscale)

Emotional Eating (TFEQ Subscale)

Three Factor Eating Questionnaire Revised 18 item

Uncontrolled Eating (TFEQ Subscale)

Urgency, Premeditation, Perseverance and Sensation Seeking

Scale

(negative) Urgency, Premeditation, Perseverance, Sensation

Seeking and Positive Urgency Scale

Visual Analogue Scale 


\section{Chapter One: Introduction and Literature Review}

\subsection{General Introduction}

Obesity is the fifth leading cause of global mortality (WHO, 2017) with more than one in two adults and one in six children thought to be obese (OCED, 2017). The prevalence of overweight and obesity has risen over the past few decades (AbarcaGómez et al., 2017; Di Cesare et al., 2016; Flegal, Carroll, Ogden, \& Johnson, 2002; $\mathrm{Ng}$ et al., 2014; Ogden et al., 2016), and whilst country specific analyses suggest that trends may be stabilising in some populations (Abarca-Gómez et al., 2017; Flegal, Carroll, Ogden, \& Curtin, 2010; Rokholm, Baker, \& Sørensen, 2010; Stamatakis, Wardle, \& Cole, 2010), obesity nevertheless causes significant economic and societal burden. For example, obesity has been associated with negative consequences to an individual's health, including an increased risk of cardiovascular disease, type 2 diabetes and cancer (Afshin et al., 2015; Douketis \& Sharma, 2005; Hruby et al., 2016; Koh-Banerjee et al., 2004; Lavie, Milani, \& Ventura, 2009). Obesity is also associated with increased healthcare utilisation and increased costs to healthcare systems (Cerny, 2017; Kent et al., 2017). Consequently, developing interventions to prevent and tackle obesity are of the upmost importance.

Whilst the causes of obesity are multifactorial, including a lack of physical activity, genetic factors, socioeconomic status, increases in energy intake, as well as psychological and environmental factors (Hruby et al., 2016; Wilding, 2001; Wright \& Aronne, 2012), overconsumption is thought to be particularly important. There is also growing consensus that overeating in response to affect, otherwise known as emotional eating, may be a significant driving factor. Indeed, recent evidence suggests 
that as many as $57 \%$ of individuals with overweight and obesity self-report frequent emotional eating (Péneau, Ménard, Méjean, Bellisle, \& Hercberg, 2013).

\subsection{Definition of Emotional Eating}

Emotional eating is commonly defined as the consumption of food in response to negative affect (Konttinen, Männistö, Sarlio-Lähteenkorva, Silventoinen, \& Haukkala, 2010; Macht \& Simons, 2000; Pike, 2013; Pinaquy, Chabrol, Simon, Louvet, \& Barbe, 2003; Thayer, 2001; van Strien \& Ouwens, 2007; van Strien, Frijters, Bergers, \& Defares, 1986). A number of different negative emotions and emotional states have been related to emotional eating, including fear, anger and sadness (Chua, Touyz, \& Hill, 2004; Macht, 1999; van Strien, Herman, Anschutz, Engels, \& de Weerth, 2012). In a large questionnaire study of participants with a wide range of ages and BMI scores, anger was more strongly related to feelings of hunger than fear and sadness (Macht, 1999). However, in a second study with a similar sample, Macht and Simons (2000) found no differences in motivation to eat between participants reporting emotional states characterised by tension, fear and sadness, with and without anger. In contrast, Mehrabian (1980) found that low arousal states (e.g. boredom, fatigue and depressive symptoms) caused an increase in food intake, whereas more highly arousing states (e.g. tension, pain and fear) reduced intake. Robbins and Fray (1980) also highlighted how different negative emotions of varying intensity may be related to emotional eating. Within college students, the tendency to experience boredom has also been shown to be predictive of emotional eating, with the effect much stronger in females (Crockett, Myhre, \& Rokke, 2015). 
With a number of overlapping features with emotional eating, binge eating has also been associated with stress in healthy females (Freeman \& Gil, 2004). For example, Crowther and colleagues (2001) found that females with normal weight who engaged in episodes of binge eating believed that their stress was more intense and emotionally distressing compared to females who did not binge eat. Daily hassles, often used as a measurement of stress, have also been significantly related to greater levels of snack consumption, and in particular, the number and intensity of daily hassles and negative affect (Newman, O’Connor, \& Conner, 2007). Taken together, the aforementioned literature suggests that a number of negative emotions, as well as stress, are associated with emotional eating.

More recently, the definition of emotional eating has also been extended to include food consumption following positive affect (Bongers, Jansen, Havermans, Roefs, \& Nederkoorn, 2013; Dingemans, Martijn, Jansen, \& van Furth, 2009; Dingemans, Martijn, van Furth, \& Jansen, 2009; Evers, Adriaanse, de Ridder, \& de Witt Huberts, 2013; Kenardy, Butler, Carter, \& Moor, 2003). Historically, research suggested that individuals were more likely to consume healthy foods in response to positive emotions, with the consumption of unhealthy foods occurring predominately during negative emotional states (Lyman, 1982). However, recent research findings suggest that this may not be the case. Bongers and colleagues (2013) grouped participants into high and low emotional eaters based on their responses to the emotional eating subscale of the Dutch Eating Behaviour Questionnaire (DEBQ; a common measure of emotional eating; van Strien, Frijters, Bergers, \& Defares, 1986). Participants rating themselves as emotional eaters consumed more food during a bogus taste test of crisps and chocolate following the induction of a positive mood compared to a neutral mood. In a food diary study, Evers and colleagues (2013) also found that 
snack intake followed positive emotions more frequently than negative emotions. Additionally, they found that participants in a positive mood induction condition consumed more calories from unhealthy snacks than participants in a control condition. When they included a negative mood induction condition, the number of calories consumed did not differ across participants in the negative and positive conditions. In comparison to the control condition, participants in the positive and negative conditions consumed significantly more calories from unhealthy snacks. These findings support the idea that increased food intake may take place in response to positive as well as negative emotions. However, for the purpose of this thesis, emotional eating will refer to the consumption of food following negative affect.

Studies often find that females report a greater tendency to emotionally eat (Lluch et al., 2000; Tanofsky, Wilfley, Spurrell, Welch, \& Brownell, 1997; Wardle, 1987; Wardle et al., 1992). Using the DEBQ, significantly higher emotional eating scores were reported by females compared to males, with the difference remaining regardless of age (i.e. similar results in children and adults; Lluch et al., 2000). In contrast, Nguyen-Rodriguez, Unger, \& Spruijt-Metz (2009) found no significant differences across sexes within a sample of adolescents. Bennett et al. (2013) conducted interviews with undergraduate students to explore the perceptions of males and females that reported themselves as high emotional eaters. Both sexes stated that they only engaged in emotional eating when experiencing negative affect, saying they ate normally during positive affect. They found that females associated their increase in food intake to experiencing states of stress. Specifically, moderate levels of stress led to an increase in consumption. In contrast, males felt that they experienced a loss of appetite when they felt stressed or were experiencing relationship difficulties. Female participants also reported feeling guilty after consuming food due to negative 
affect, whereas male participants only reported feelings of guilt when they felt their consumption was an overconsumption of food or portion size. Furthermore, when exploring the influence of food on emotional states, Kenardy et al. (2003) found both sexes reported a significant reduction in negative affect following food consumption, whereas only females reported an increase in positive affect. Therefore, it could be that females are more likely to engage in emotional eating in general, using it as a method for increasing positive affect.

\subsection{Emotional Eating and Body Mass Index}

Emotional eating has been associated with changes in weight/BMI, weight gain and increased BMI (Blair, Lewis, \& Booth, 1990; Braet \& van Strien, 1997; Fox, Conneely, \& Egan, 2017; Geliebter \& Aversa, 2003; Greene et al., 2011; Keller \& Siegrist, 2015; Koenders \& van Strien, 2011; Lluch et al., 2000; Sung, Lee, \& Song, 2009; Tapper et al., 2009; van Strien, Herman, \& Verheijden, 2009; van Strien, Peter Herman, \& Verheijden, 2012; Wardle, 1987), particularly in individuals that experience high levels of stress (Torres \& Nowson, 2007). Typically, emotional eating is measured through self-report questionnaires which ask participants to indicate the extent they eat or feel the urge to eat when experiencing emotional arousal (e.g. DEBQ, Emotional Eating Scale; EES; Arnow, Kenardy, \& Agras, 1995, and the Three Factor Eating Questionnaire; TFEQ; Stunkard \& Messick, 1985). Individuals that self-report as high emotional eaters on such measures have been found to be at a greater risk of becoming overweight or obese (Sung et al., 2009; van Strien, Herman, et al., 2012) and are also more likely to overeat when faced with hunger and emotional experiences (Adriaanse, de Ridder, \& Evers, 2011). 
Research findings from longitudinal studies also provide evidence for a significant relationship between emotional eating and BMI. For example, in a prospective study of bank employees in the Netherlands, participants with higher BMI scores reported higher levels of emotional eating, as well as higher levels of dietary restraint and external eating (Koenders \& van Strien, 2011). High emotional eating contributed to an increase in BMI after two years. External eating and restrained eating (eating in response to food related stimuli regardless of internal states of hunger and individuals who limit food intake to maintain or reduce body weight and shape respectively; van Strien et al., 1986) did not influence the change in BMI, while physical exercise and engaging in sports contributed to a decrease in BMI. Hierarchical regressions revealed that emotional eating was the only significant predictor of increased BMI (Koenders \& van Strien, 2011). In a later study, emotional eating was found to moderate the association between over-consumption and weight gain (van Strien, Herman, et al., 2012). A prospective study across two years found overconsumption was more strongly related to higher levels of emotional eating, and external eating and was also significantly associated with weight gain. Consistent with this, similar findings have been reported in a representative sample of Korean twins and their families (Sung et al., 2009). Specifically, Sung and colleagues (2009) found that emotional eating was significantly related to weight gain over four years and current BMI. However, it should be noted that the study used retrospective selfreported weight of four years previously to calculate weight change.

Additionally, in a large study of individuals with normal weight and overweight, van Strien et al. (2009) also found a significant association between higher levels of emotional eating and BMI. Specifically, they found that emotional eating was significantly associated with over-consumption. Therefore, it may be that individuals 
who report themselves as emotional eaters are vulnerable to overconsumption, and in turn, increased BMI. In a similar representative Dutch sample over 20 years previous, van Strien et al. (1986) found much lower levels of emotional eating, possibly indicating that there has been an increase in emotional eating scores across the last two decades. As previously mentioned in the opening section of this chapter, the prevalence of overweight and obesity has risen over the last few decades. Thus, emotional eating could be a contributing factor.

Indeed, it is possible that engaging in emotional eating causes a cycle to develop where individuals repeatedly turn to food. After engaging in emotional eating and initially feeling better, individuals who have overeaten or consumed food they do not usually consume (due to high fat, sugar or salt content) may feel guilty for doing so (Dubé, LeBel, \& Lu, 2005; Macht \& Dettmer, 2006), especially if they are restrained eaters and have previously been 'good'. In turn, such individuals may return to food consumption in an attempt to alleviate these new sensations of guilt, and from this, a vicious cycle is born. This cycle of continuous over consumption of foods may increase BMI, and in turn, have a negative impact on health. However, despite this and several studies highlighting an association between emotional eating and BMI and/or weight gain, the nature and causal direction of the association remains unclear. For example, it could be that emotional eating leads to increased weight gain and in turn BMI, or alternatively, it could be that emotional eating is a consequence of a higher BMI and weight gain. Either way, the available research supports the need to add to the knowledge base on emotional eating and BMI, especially considering the societal and health related burden of obesity.

A third possibility is that BMI and emotional eating are unrelated constructs, a proposal that has been supported by the findings of numerous research studies 
(Adriaanse et al., 2011; Appelhans, Whited, Schneider, Oleski, \& Pagoto, 2011; Wardle et al., 1992). For example, even though Adriaanse and colleagues (2011) did not specifically explore the association between emotional eating and BMI, they failed to find a significant relationship between BMI and emotional eating in two of their studies. However, in both of their samples, individuals had predominantly healthy BMI scores with very little range which may have influenced the results. This may provide an explanation of the differences in findings in comparison to Koenders \& van Strien (2011), van Strien et al. (2009) and van Strien et al. (2012) who used a much broader BMI range.

Within a sample of adolescents, Wardle and colleagues (1992) also found no significant association between BMI and emotional eating even though emotional eaters tended to feel 'fatter'. Likewise, similar levels of emotional eating were found in adolescents with normal weight and overweight BMI scores (Nguyen-Rodriguez, Chou, Unger, \& Spruijt-Metz, 2008). Similarly, no significant association between emotional eating and being overweight was found in adolescent girls, but there was a significant negative association in boys (Snoek, van Strien, Janssens, \& Engels, 2007). However, it may be that emotional eating develops over time. Indeed, significant associations between emotional eating and BMI have been found in representative samples of adults, but not in adolescent and student samples. Therefore, it may be that the association between emotional eating and BMI gets stronger with age, which may help to explain different findings across adolescent and adult samples. It is possible that emotional eating may develop over time and its impact does not have an effect when food intake is controlled by parents for adolescents (Snoek, Engels, Janssens, \& van Strien, 2007) or whilst students face financial difficulty. 


\subsubsection{Emotional eating and food intake}

One possible explanation for the inconsistent associations between emotional eating and BMI may rest in the lack of concordance between self-reported emotional eating and actual food intake behaviour. Similar to the research on BMI, whether the presence of emotional eating results in an actual increase in food intake is varied (Lluch et al., 2000; Newman et al., 2007; van Strien et al., 2007; Wardle et al., 1992). Some studies have shown that those who report high levels of self-reported emotional eating experience an increase in food intake (Newman et al., 2007; van Strien et al., 2007). For instance, Newman and colleagues (2007) used the TFEQ and the DEBQ to assess emotional eating, finding that snack intake was significantly associated with higher levels of emotional eating. They also found that cognitive restraint and disinhibition (a lack of control in the consumption of food; Stunkard \& Messick, 1985) significantly predicted snack intake. This highlights that even though emotional eating may be linked to food consumption, other factors may also be important. In contrast, Wardle et al. (1992) found no significant association between emotional eating and food intake in a sample of adolescents, and Lluch et al. (2000) found no significant association between levels of emotional eating (as measured by the DEBQ) and food intake (food diaries) within a sample of male and female children and adults.

One possible explanation for the inconsistent associations between emotional eating and BMI is the 'triple recall bias'. This bias, proposed by Evers and colleagues (2009), relates to the self-report nature of emotional eating. Individuals are expected to recall their experiences of negative emotions, their eating behaviour in response to these emotions, as well as create associations between the two. Therefore, it may be that the self-report measures only assess an individual's perceived association between 
their emotions and eating behaviours (Bekker, van de Meerendonk, \& Mollerus, 2004). In particular, self-report measures only assess an individual's beliefs about how often they eat when they are emotional (Lluch et al., 2000) and how comforting they find eating when experiencing emotions (Spoor, Bekker, van Strien, \& van Heck, 2007). Adriaanse et al. (2011) suggested that a lack of self-awareness concerning the extent of an individual's engagement in emotional eating may be due to the habitual nature of eating. For example, snacking is often undertaken without thinking, which may result in individuals being unable to accurately distinguish when they eat in response to emotions. This is based on the idea that habitual behaviours are often performed outside of conscious awareness (Aarts \& Dijksterhuis, 2000). Within two studies exploring female undergraduate students, unhealthy snack intake was explained by habit and restraint eating, but not emotional eating (Adriaanse et al., 2011). To check it was not the consumption of a specific category of snacks (i.e. healthy versus unhealthy) that was related to self-reported emotional eating, Adriaanse and colleagues (2011) conducted a regression analysis on the consumption of healthy snacks. This revealed that individuals who reported themselves as low external eaters consumed more healthy snacks. They suggested that individuals may habitually snack when emotional but are not aware of the link as it is performed outside of conscious awareness. With this in mind, the authors wanted to explore the beliefs surrounding those who reported themselves as emotional eaters. In a series of regression analyses, emotional eating was a significant predictor of worrying about one's eating behaviour, greater monitoring of eating behaviour, lower perceived control over eating behaviours, and a stronger extrinsic motivation for healthy eating behaviour. These findings highlight that emotional eaters have increased concerns regarding their eating behaviours, and therefore, it may be that emotional eaters experience more negative 
affect, they misinterpret their feelings, or they have an increased general focus on their eating behaviours and believe they consume more when emotional (Adriaanse et al., 2011).

\subsection{Theories of emotional eating}

Given the role emotional eating may play in obesity, numerous theories have been developed to explain the phenomenon. Previously, when experiencing highly arousing negative emotions a reduction in appetite was observed (Greeno \& Wing, 1994). Based on the idea of fight or flight, when individuals are highly aroused, a loss of appetite is experienced due to the focus on preparing the muscles for action, instead of on gastrointestinal and other organ functions. It has been suggested that for acute levels of stress and negative affect, a reduction or total loss of appetite is experienced (Torres \& Nowson, 2007). However, as highlighted in section 1.2., there are also instances where increased food intake occurs following positive affect.

Other theories place an emphasis on the possible role of poor self-esteem, low levels of basic need of satisfaction (Timmerman \& Acton, 2001), as well as parental influence and upbringing (Wardle, Sanderson, Guthrie, Rapoport, \& Plomin, 2002; Snoek et al., 2007; Topham et al., 2001; van Strien \& Bazelier, 2007). Timmerman and Acton (2001) found that scores on the basic need satisfaction inventory (BNSI; Leidy, 1994) were significantly correlated with emotional eating, suggesting that a lower level basic need of satisfaction is associated with a greater likelihood of engaging in emotional eating due to a lack of basic needs acting as a stressor. Snoek and colleagues (2007) found that higher levels of parental emotional eating were associated with higher levels of emotional eating in adolescents, suggesting that 
parents act as models and demonstrate emotional eating to their children. When further exploring parenting style, they found that higher perceived behavioural control was associated with greater levels of emotional eating in both younger (7-9 years) and older (10-12 years) children. Finally, perceived restriction was more strongly associated with lower levels of emotional eating in younger than older children (van Strien \& Bazelier, 2007). For boys, a perceived pressure to eat was significantly related to higher levels of emotional eating, but the same association was not found in girls. In this study, van Strien and Bazelier (2007) found low levels of emotional eating in children of both sexes, implying that children may show a loss of appetite to emotions, which is the natural response, and that emotional eating may develop in later life through other mechanisms.

In addition, there are also a group of theories available to explain emotional eating that focus on the management of emotions as being fundamental to the development of emotional eating. Specifically, they argue that a potential cause of emotional eating is how emotions are regulated instead of the emotions being experienced per se (Wiser \& Telch, 1999). Consistent with this, Evers et al. (2010) found that negative emotion did not predict emotional eating, but instead, was predicted by coping strategies adopted following negative emotion. The authors further proposed that emotional eating may not simply reflect eating in response to negative emotions, but instead, an inability to effectively regulate emotions. When an individual engages in emotional eating, there are a range of existing theories which incorporate emotional regulation to help to explain such behaviours.

First, emotional eating may be an attempt to escape from negative emotion (Heatherton \& Baumeister, 1991; Macht, Gerer, \& Ellgring, 2003; Spoor et al., 2007). Heatherton and Baumeister (1991) argued that individuals may engage in emotional 
eating to escape and avoid negative emotion. Specifically, emotional eating may allow individuals to narrow their focus and avoid having to deal with such emotions. In support of this, Spoor et al. (2007) found that emotional eating was related to avoidance distraction and emotion-oriented coping in females with and without eating disorders.

Second, food consumption may alleviate the experience of negative emotions by producing and allowing positive emotions to be experienced instead (Christensen, 2001; Lehman \& Rodin, 1989). Support for this theory is provided by Macht and Mueller (2007). In this study, participants were offered pieces of chocolate or given water after a negative mood induction, with negative mood immediately improving following consumption of chocolate. In addition, individuals often turn to foods they do not regularly consume following negative emotions as a 'treat' to themselves (Fairburn \& Cooper, 1982). This 'treat' serves as a way of making them feel better. Restrained eaters are said to be more vulnerable to engaging in emotional eating due to being unable to cognitively balance their usual cognitive restraint and emotions (Greeno \& Wing, 1994). This may result in restraint eaters consuming 'banned' foods as a 'treat' because they are unable to follow their usual rules.

Third, emotional eating may offer a way to mask emotions and to provide distraction (Bennett et al., 2013; Polivy \& Herman, 1999). Polivy and Herman (1999) examined whether participants use overeating to mask distress by leading female students to believe they had either failed or successfully completed a cognitive task followed by an ab-libtum ice cream taste test. Compared to unrestrained eaters, restrained eaters were significantly more likely to attribute their negative feelings during the experiment to the ice cream, and even more so following 'failing' the task. This suggests that participants may have masked their feelings, believing instead that 
the cause of their increased negative affect was due to the consumption of ice cream rather than task failure. Additionally, Bennett et al. (2013) found that using emotional eating as a distraction tool was a common theme in a qualitative study exploring individual's perceptions of emotional eating.

Fourth, emotional eating may occur as a result of poor emotional regulation strategies (e.g. emotional suppression; Evers, Stok, \& Ridder, 2010). Amongst female students across three studies, emotional suppression was found to be associated with increased food intake following manipulated suppression and through a self-report measure of emotional suppression (Evers et al., 2010). Finally, Bruch (1973) conceptualised that emotional eating was derived from some individuals being unable to differentiate hunger from the bodily sensations associated with negative emotions. Specifically, the psychosomatic theory proposes that emotional eating is caused by individuals being discriminately unaware of their bodily sensations and an inability to control their emotional functions or needs because they do not recognise them. For example, individuals may experience temporary relief from feelings of hunger by turning to food, but as these sensations are not interpreted accurately as symptoms of anxiety and depression, the underlying cause of them is not addressed. As a result, they could find themselves in a cycle of unsatisfying eating behaviour. Additionally, individuals who engage in emotional eating may not have developed the ability to appropriately perceive and cope with their emotions. Therefore, food is used as a coping strategy when complex emotions are experienced. Bruch (1973) stated that a child should learn to correctly identify their bodily sensations and needs to be able to satisfy them in way that is biologically, as well as socially and culturally acceptable. Bruch (1973) also argued that some individuals are unable to internally process the need for food and instead rely heavily on external cues. 
All of the theories presented in this section argue that emotional eating is not a response to the experience of negative emotions but is a consequence of how emotions are regulated. These theories propose that being unable to adaptively respond to emotions results in emotional eating. As turning to food during periods of negative emotion has previously been an effective strategy, individuals consistently return to the consumption of food to make themselves feel better. Frequent engagement in emotional eating is likely to cause weight gain over time, and therefore, if individuals are going to successfully manage their weight they need to be able to regulate their emotions. Key to this is their ability to understand, comprehend and perceive emotions which can be encapsulated by the term 'emotional regulation'. Emotional regulation is defined as the intentional or automatic strategies that influence our experience of emotions in terms of type (Gross \& Barrett, 2011). Emotional regulation can lead to the development of behaviour in response to situational demands, and if maladaptive, can interfere with functioning (Cole, Michel, \& Teti, 1994). When such behaviour occurs, it is typically referred to as emotional dysregulation. Emotional dysregulation, involving the combination of emotional vulnerability and an inability to modulate emotional responses (Gunderson \& Zanarini, 1989; Linehan, 1993; 1995), is thought to encompass three elements: difficulty identifying and describing emotions, the regulation of emotions, and the consequent behaviours, such as emotional eating (Spence \& Courbasson, 2012). The examination of emotional dysregulation as a mechanism underpinning emotional eating is the basis of this $\mathrm{PhD}$ thesis. 


\subsection{Emotional dysregulation}

\subsubsection{Emotional dysregulation and eating behaviours}

Despite receiving little attention in the research literature, initial evidence suggests that emotional dysregulation may play an important role in eating disorders. When comparing females with a history of eating disorders, and in particular anorexia nervosa (AN), to a control group of healthy females, the former group were shown to have significantly higher levels of emotional dysregulation (Holliday, Uher, Landau, Collier, \& Treasure, 2006). Holliday and colleagues (2006) found that levels of personality traits (including emotional dysregulation) associated with eating disorders did not differ significantly between females with acute AN and those with remitted AN. This suggests that emotional dysregulation is a persistent feature of AN that may influence eating behaviour. Nevertheless, there are challenges in generalising findings from a clinical population. Hence, there is a need to expand the current literature base.

Poor emotional regulation and emotional awareness have been associated with loss of control eating (LOC) in female adolescents (Goldschmidt, Lavender, Hipwell, Stepp, \& Keenan, 2017). In a longitudinal study, Goldschmidt and colleagues (2017) found evidence to suggest that deficient emotional regulation may contribute to the onset and maintenance of LOC. However, as effect sizes were small, the authors suggested that additional factors were likely to play a role. They suggested negative urgency may be a factor to consider and this construct is explored later in Chapter One. Although LOC is different from emotional eating, it nonetheless has several overlapping features. Consequently, it is logical to theorise that if there is an 
association between emotional dysregulation and LOC, a relationship between emotional dysregulation and emotional eating may also exist.

Additionally, emotional dysregulation mediated the relationship between insecure attachment and disordered eating in a sample of healthy undergraduate students (Han \& Kahn, 2017). Han and Kahn (2017) found that both binge and restraint eating were influenced by attachment avoidance and attachment anxiety via emotional dysregulation. Notably, the associations with binge eating could be suggestive of the possible relationship between emotional dysregulation and emotional eating due to the similarity of behaviours.

Focusing on emotional eating specifically, Fox et al. (2017) found that believing emotion was a sign of weakness (subscale of the Attitudes towards Emotional Expression; AEE; Laghai \& Joseph, 2000) was the strongest predictor of emotional eating. In addition, the belief that emotions should be controlled (AEE subscale) had the strongest effect on BMI. These findings suggest that the way emotional expression is perceived by individuals has important consequences for emotional eating. The authors conclude that negative affect becomes a 'trigger' to emotional eating and emotional regulation plays a prominent role; providing further support for the contributing role of emotional dysregulation in emotional eating. Overall, it is proposed that individuals who engage in emotional eating have fewer strategies to regulate their emotions and are less able to control their experience of negative emotions as a result. In turn, they may turn to food, overeating to alleviate their negative emotions because it has previously been a successful strategy. 


\subsubsection{Emotional Dysregulation and Body Mass Index}

Even though evidence suggests a relationship between emotional dysregulation and emotional eating, little research has explored whether this association extends to BMI. Moghaddam and BabaKhani (2016) found that females with obesity scored significantly higher on three aspects of the difficulties in emotion scale (DES; Gratz \& Roemer, 2004) in comparison to females with a healthy BMI. Specifically, females with obesity reported a greater lack of emotional transparency and emotional awareness, as well as a limited access to emotional regulation strategies. In addition, self-reported emotional eating was significantly higher in the obese compared to healthy weight group. Across both groups, a significant negative relationship was found between emotional regulation and emotional eating. That is, lower levels of emotional eating were associated with better emotional regulation abilities. In addition, emotion regulation, along with incompatible problem solving (i.e. negative problem solving orientation and avoidance problem solving styles; social problem solving skills; D’Zurilla, Nezu, \& Maydeu-Olivares, 2002), significantly predicted the tendency to emotionally eat in both groups. Although, the effect was stronger in the obese rather than healthy weight group.

In a laboratory setting, Appelhans and colleagues (2011) used an experimental paradigm involving distraction techniques to further explore differences in food intake. Snack consumption following an angry or neutral mood induction did not significantly differ in individuals with either normal weight or obesity. However, when they explored the role of distraction strategies, individuals with obesity who used less distraction strategies consumed more in the anger manipulation compared to the neutral condition. They suggested that the relationship between BMI and emotional 
eating was somewhat unclear, but suggested that individuals who have a limited use of distraction strategies may become obese over time as they have grown reliant on emotional eating (Appelhans et al., 2011). It is also possible that such individuals emotionally eat as a distraction strategy, where focusing on food distracts from their experience of negative moods such as anger. However, as the use of distraction strategies did not influence food intake in individuals with normal weight, it seems likely that additional factors may be involved in the relationship between emotional dysregulation and BMI.

\subsubsection{Interim summary}

The literature presented so far suggests that the way individuals manage their experience of negative emotions may be a key factor underpinning emotional eating, helping to explain why some individuals engage in emotional eating and others do not. It may be that individuals turn to food as a means of escaping or masking their emotional distress (as examples). This occurs when an individual lacks the skills needed to regulate negative emotions and instead, utilise maladaptive emotion regulation strategies (Stepp et al., 2014). When turning to food alleviates the negative sensations associated with negative affect, the association is strengthened and individuals will continue to use maladaptive emotion regulation strategies (Goldschmidt et al., 2017). Available research suggests that emotional dysregulation is associated with both eating disorders and disordered eating behaviours, and that certain beliefs about emotions can predict emotional eating. Given this, it seems plausible to suggest that emotional dysregulation may play a role more broadly in eating behaviours, and specifically, emotional eating. However, this has yet to be 
comprehensively examined. Emotional dysregulation involves three elements; a) the ability to appropriately identify and describe emotions; b) the ability to regulate emotions, and c) the consequent behaviours as an attempt to regulate emotions, such as emotional eating (Spence \& Courbasson, 2012). As difficulty identifying and describing emotions are two elements that characterise both alexithymia and emotional dysregulation, alexithymia could be used as a proxy of emotional dysregulation. Therefore, exploring the influence of alexithymia on emotional eating should prove a useful strategy for improving our understanding of the role of emotional dysregulation in emotional eating.

\subsection{Alexithymia}

Alexithymia is a normally distributed personality trait characterised by difficulties identifying and verbalising feelings (Bagby, Parker, \& Taylor, 1994; Mattila, Salminen, Nummi, \& Joukamaa, 2006; Sifneos, 1973). In particular, individuals with alexithymia experience difficulty identifying feelings and distinguishing them from the somatic sensations accompanying emotion (DIF), difficulty describing feelings to other people (DDF), constricted imaginal processes, and a stimulus-bound, externally oriented thinking style (EOT; Sifneos, 1973; Taylor, Bagby, \& Parker, 1997). Two sub-types of alexithymia have been defined within the literature; type I includes the absence of the emotional experience followed by the absence of emotional cognition, and type II includes a selective deficit of emotional cognition with sparing of emotional experience (Vorst \& Bermond, 2001).

Alexithymia has been classified as a disorder of affect regulation (Ruth \& Padmakumari, 2014), a stable trait, as well as a dynamic and multifaceted construct 
(Lumley, Neely, \& Burger, 2007). Alexithymia has been shown to be highly prevalent in a number of medical and clinical conditions, particularly those characterised by poor affect regulation and the use of maladaptive coping strategies (e.g. Kang, Namkoong, Yoo, Jhung, \& Kim, 2012; Shishido, Gaher, \& Simons, 2013). For this reason, alexithymia may also be associated with emotional eating as another maladaptive coping strategy.

Alexithymia is strongly associated with emotional intelligence, defined as an individual's ability to monitor one's own and others' feelings and emotions, to discriminate among them, and to use this information to guides one's thinking and actions (Salovey \& Mayer, 1990). However, despite their overlapping features and strong correlations between the Toronto Alexithymia Scale (TAS-20; Bagby, Parker, et al., 1994) and EQ-I (a measure of emotional intelligence; Bar-On, 2004), Parker, Taylor, and Bagby (2001) argue that they should be considered separate constructs. Exploring the relationship between emotional intelligence and alexithymia in greater detail, it appears alexithymia is most strongly associated with the adaptability and stress management subscales of the EQ-I. That is, maladaptive defence and coping strategies (Parker, Taylor, \& Bagby, 1998). Moreover, Panayiotou and colleagues (2015) found that in both students and a clinical population with Obsessive Compulsive Disorder (OCD; DSM-5; American Psychiatric Association, 2013), alexithymia was significantly correlated with emotional avoidance. Individuals with alexithymia may have learnt over time that avoiding emotions and preventing themselves from experiencing emotions is a good method of overcoming their difficulties with emotion (e.g. identifying emotions). They argue that individuals with alexithymia do not lack the ability to process emotions but have not learnt to do so in an adaptive manner, developing maladaptive coping strategies as a result. 
Consequently, individuals with alexithymia avoid experiencing unwanted or unpleasant emotions. These studies highlight the considerable overlap between alexithymia and emotional dysregulation. Furthermore, evidence for the convergence of alexithymia and emotional dysregulation is provided across a range of studies (e.g. Stasiewicz et al., 2012; Swart, Kortekaas, \& Aleman, 2009; Taylor, 2000), providing further support for the notion that alexithymia is an appropriate construct to capture emotional dysregulation.

Commonly viewed as being on a continuum in the general population (Mikolajczak \& Luminet, 2006; Salminen, Saarijärvi, Äärelä, Toikka, \& Kauhanen, 1999), alexithymia is more prevalent in males than females (Honkalampi, Hintikka, Tanskanen, Lehtonen, \& Viinamäki, 2000; Lane et al., 1996; Lane, Sechrest, \& Riedel, 1998; Mattila et al., 2006; Parker, Taylor, \& Bagby, 2001). Although, some studies have found that males can identify their emotions as well as their female counterparts, but experience greater difficulty describing and expressing their feelings (Parker, Taylor, \& Bagby, 2003; Salminen et al., 1999). In contrast, other research has found notable differences across sexes on the EOT subscale of the TAS-20 (Parker et al., 2001). Specifically, males tend to focus on external problems instead of internal feelings and emotions. One suggestion for the differences across sexes is the selfreported nature of alexithymia. Individuals may not be aware of their difficulties in comprehending emotions but may be accurate in assessing the externally oriented thinking elements. Therefore, at high levels of alexithymia, individuals may be able to rate themselves based on a skill or habit when exploring externally oriented thinking, but are unable to recognise how well they are at identifying and expressing emotions (Lane et al., 1998). Furthermore, females report experiencing more positive (Wood, Rhodes, \& Whelan, 1989) and negative (Mirowsky \& Ross, 1995) emotions compared 
to males, as well as greater emotional awareness overall. As a result, females may have developed better ways to identify and describe emotions due to experiencing them more frequently than males.

Greater levels of alexithymia have also been associated with increased age (Honkalampi et al., 2000; Lane et al., 1996, 1998; Mattila et al., 2006). These associations could be related to differences in the upbringing of younger and older generations (Mattila et al., 2006) or processing changes that occur as we age (Calder et al., 2003; Fischer et al., 2005; Gunning-Dixon et al., 2003). Inconsistent with this, other studies which have found no significant associations between alexithymia and age (Bach, Bach, Böhmer, \& Nutzinger, 1994; Parker, Taylor, \& Bagby, 1989). Alexithymia has also been significantly associated with lower levels of education (Honkalampi et al., 2000; Mattila et al., 2006; Todarello, Taylor, Parker, \& Fanelli, 1995) and lower socio-economic status (SES; Lane et al., 1996, 1998); in-line with a strong cognitive component in emotional awareness which can be influenced by the environment (e.g. such as educational background). Poorer health outcomes and a greater number of physical complaints have also been associated with alexithymia (Bach et al., 1994). It is possible that lower SES and education levels could devalue the benefits of expressing negative emotions with others, causing symptoms of low affect to be presented physically, as well as increasing the likelihood of maladaptive coping behaviours occurring (e.g. substance abuse; Lane et al., 1998) which lead to additional physical complaints. 


\subsubsection{Alexithymia and eating behaviour}

Alexithymia has been associated with a variety of eating disorders, including AN and bulimia nervosa (BN; Beales \& Dolton, 2000; Berthoz, Perdereau, Godart, Corcos, \& Haviland, 2007; Cochrane, Brewerton, Wilson, \& Hodges, 1993; Corcos et al., 2000). In their review of the associations between alexithymia and eating disorders, Morie and Ridout (2018) noted consistently higher levels of alexithymia (DIF and DDF) in individuals with eating disorders compared to those without. In contrast, EOT subscale scores only differed between those with and without AN (see Abbate-Daga et al., 2015, as cited in Morie \& Ridout, 2018). Therefore, owing to the associations between alexithymia and eating behaviours in a clinical setting, it is possible that alexithymia is also associated with emotional eating. Reports have linked emotional eating with difficulties in identifying and expressing emotions, which is a core element of alexithymia. Spence \& Courbasson (2012) found that greater difficulty identifying and describing emotions gave rise to participants feeling less able to regulate their emotions. This resulted in the use of poor coping expectancies, which in turn, predicted emotional eating. They also found evidence for the EOT subscale of the TAS-20; specifically, participants who focused on external stimuli rather than emotions felt more capable of regulating their mood. Consistent with this, Larsen, van Strien, Eisinga, \& Engels (2006) found that higher levels of emotional eating were significantly associated with higher levels of alexithymia (specifically DIF and DDF) in a sample of 140 individuals with obesity. Furthermore, stronger correlations between alexithymia and emotional eating were found in males compared to females. Whilst levels of emotional eating remained significantly higher in females regardless of levels of alexithymia, when males reported higher levels of DIF and DDF, an 
increase in emotional eating was seen. Overall, they argued that alexithymic characteristics were more strongly involved in emotional eating in males with obesity compared to females with obesity.

Studies have also investigated how stable alexithymia is in eating disorders over time and following treatment (for a review, see Morie \& Ridout, 2018). In a sample of individuals with AN and BN, total and DIF alexithymia scores decreased over time alongside treatment (Sexton, Sunday, Hurt, \& Halmi, 1998), but the decline was also matched with a decrease in depression. The DDF subscale did not decrease over the course of treatment, theorising that this subscale, difficulty expressing one's feelings to others, is a stable construct. Both Larsen et al. (2006) and Sexton et al. (1998) suggest that depression plays an important role in the relationship between alexithymia and eating behaviours, which will be explored in greater detail later in Chapter One.

Additionally, higher levels of alexithymia have been found in individuals with binge eating disorder (BED) compared to individuals without (Pinaquy et al., 2003). Pinaquy and colleagues (2003) found evidence to suggest that alexithymia scores predicted eating in response to emotions (17\% total variance explained) and specifically, the DIF subscale was a significant predictor. Sifneos (1996) argued that individuals with alexithymia prefer to act instead of talking about feelings, evident here by individuals turning to food. Pinaquy and colleagues (2003) further suggested that whilst alexithymia is secondary to depression in individuals without BED, it may serve as the primary condition in individuals with BED as there is an absence of a relationship between alexithymia and depression in this subgroup. In contrast, in a sample of adult outpatients with BED, the presence of alexithymia and depression was 
associated with increased psychopathology, and specifically, suicide ideation (Carano et al., 2012).

Outside of clinical samples, significant associations between alexithymia and eating behaviours have also been found. For example, Karukivi and colleagues (2010) explored the relationship between the TAS-20 and the SCOFF ("Sick, Control, One, Fat, Food" questionnaire; Morgan, Reid, \& Lacey, 1999), finding a significant positive relationship between scores in a sample of healthy adolescents. Eating behaviour symptoms, where a higher score on the SCOFF questionnaire is indicative of a risk for eating disorders, were more common in individuals scoring higher on the TAS-20. Furthermore, the DIF and DDF subscales were found to be driving the association. The study concluded that adolescents who display alexithymic traits are at risk of developing eating disorders in the future. Further, Nowakowski, McFarlane and Cassin (2013) also found higher levels of alexithymia (notably DIF and DDF and not EOT) to be associated with greater disordered eating behaviour in their review of findings obtained in non-clinical undergraduate student samples. Overall, such findings support the rationale for, and importance of, exploring the relationship between alexithymia and emotional eating within healthy, non-clinical populations.

Within the laboratory, experimental studies have also explored the associations between emotional eating and emotional recognition deficits. For example, in a sample of healthy female students, those with higher levels of disordered eating (as measured by the Eating Disorders Inventory; EDI-2; Garner, 1991) correctly identified fewer emotional facial expressions (primary emotions as presented in video clips) than those who had lower levels of disordered eating (Ridout, Thom, \& Wallis, 2010). Ridout et al. (2010) also found significantly higher levels of alexithymia, depression and trait anxiety in the high compared to low EDI group. Therefore, it appears that individuals 
who are unable to correctly identify emotional expressions are more likely to experience emotional dysregulation through a reduced ability to identify the emotions of others. This could lead to personal conflicts and reluctance to share emotional experiences, impacting upon maladaptive eating behaviours. Further examination revealed that alexithymia and bulimia accounted for approximately $36 \%$ of the variance in emotion recognition accuracy. Such results provide evidence for the associations between alexithymia and disordered eating in subclinical samples, raising the possibility that associations may be generalisable to emotional eating in nonclinical populations. Consistent with this, similar findings have also been reported more recently by Ridout and colleagues (2012) using static faces, and by Alpaslan et al. (2015).

In contrast, Noli and colleagues (2010) found a similar frequency of emotional eating in a sample of severely obese individuals with and without alexithymia, suggesting that alexithymia is independent of emotional eating. However, they also found elevated levels of cognitive restraint, disinhibition and hunger in those with alexithymia, indicating the presence of some degree of disordered eating behaviour in that group. It is possible that the use of an invalidated measure of emotional eating and the highly specific and complex sample studied (individuals with obesity either waiting for or following bariatric surgery), accounts, at least in part, for these mixed findings.

\subsubsection{Alexithymia and BMI}

Even though studies often report collecting relevant data, the relationship between alexithymia and BMI has rarely been explored (e.g. Ouwens, van Strien, \& 
van Leeuwe, 2009; Pinaquy et al., 2003; Speranza et al., 2005). However, from the limited studies that have described the relationship, findings appear to be inconsistent. For instance, Zeeck and colleagues (2011) found that alexithymia scores differed across individuals with obesity and healthy BMI scores, but only in individuals with BED. Furthermore, Carano et al. (2006) found that individuals with alexithymia had significantly higher BMI scores compared to individuals without (within a clinical population), and that the prevalence of obesity (BMI score $\geq 30$ ) was significantly higher in the group with alexithymia.

In contrast, in a sample of female students, no significant difference in BMI was found between individuals with and without alexithymia (Sasai, Tanaka, \& Hishimoto, 2010). Similarly, alexithymia total, DIF and DDF subscales scores were not significantly related to BMI in a sample of individuals with AN (Torres et al., 2015). Unfortunately, Torres and collegues (2015) did not report the correlations between alexithymia and BMI for the healthy control group. Within healthy young males, Helmers \& Mente (1999) found no significant association between alexithymia (TAS-26; Taylor, Ryan, \& Bagby, 1985) and self-reported BMI, although an association was found with poor nutritional health habits. Zak-Golab et al. (2013) also found similar levels of alexithymia across participants with obesity, with or without BED. In addition, a recent meta-analysis found elevated levels of DIF and EOT, but not DDF, in individuals with obesity compared with controls (Fernandes, FerreriaSantos, Miller, \& Torres, 2018). Therefore, whilst there appears to be some empirical evidence for a role of alexithymia in emotional eating and BMI, findings are mixed and inconsistent across similar samples. 


\subsubsection{Interim Summary}

Alexithymia is an important element of emotional dysregulation and is characterised by difficulty understanding emotions and a preference for focusing on external stimuli. A plethora of research highlights that alexithymia is highly prevalent in eating disorders and is associated with various aspects of disordered eating. The majority of research points to the DIF and DDF aspects of alexithymia having the greatest relationship with disordered eating, whilst DIF and EOT subscales appear to be more strongly related to obesity and BMI. Alexithymia can therefore be used as a correlate of emotional dysregulation, offering a useful framework in which to explore the underlying mechanisms of emotional eating. However, whilst there seems to be some empirical findings in support of a relationship between emotional eating and alexithymia, the picture is somewhat mixed. This suggests that other factors may need to be considered alongside and based on available literature; impulsivity could be a theoretically driven mediating factor.

\subsection{Impulsivity}

Impulsivity is defined as the tendency to think and plan insufficiently without reflection which can result in maladaptive action without consideration of the consequences (Dawe \& Loxton, 2004; Girdhar, Mital, Kephart, \& Young, 2001; Guerrieri, Nederkoorn, \& Jansen, 2007; Guerrieri, Nederkoorn, Stankiewicz, et al., 2007; Moeller, Barratt, Dougherty, Schmitz, \& Swann, 2001). Numerous conceptualisations of impulsivity exist, including inhibitory control (ability to stop a response that is no longer required or appropriate; e.g. Logan, Schachar, \& Tannock, 
1997), delayed gratification (ability to focus on a larger, future goal; Ainslie, 1975; Rachlin, 1995), and rash spontaneity (responding without adequate thought; Dawe \& Loxton, 2004). In addition, four distinct facets have been identified when exploring impulsivity alongside the five factor model of personality (Whiteside \& Lynam, 2001). The facets derived were urgency (acting rashly in response to affect), premeditation (lack of; a reduced tendency to delay action and to carefully think and plan), perseverance (lack of; ability to remain focussed on a task, especially when it is difficult or boring) and sensation seeking (the tendency to seek excitement and take risks). More recently, urgency was split into positive urgency and negative urgency to reflect the difference in response to positive and negative affect respectively (Cyders et al., 2007). Delayed discounting is the first concept of impulsivity that will be addressed in this thesis. Second, the concept of negative urgency will also be explored.

Delay discounting, or temporal discounting, refers to a behavioural-economic concept that means as you delay a consequence or reward, its effect on behaviour decreases (Critchfield \& Kollins, 2001). Otherwise known as delayed gratification (as previously mentioned above), it is a relatively stable trait (Odum, 2011). Highly impulsive individuals have the tendency to choose a smaller, more immediate reward over a larger, more delayed reward (Ainslie, 1975; Rachlin, 1995). The devaluation of delayed consequences can be seen that when all else is equal, sooner rewards and reinforcers are preferred over delayed rewards, and delayed punishments or losses are preferred over sooner ones (Johnson, 2012). In essence, immediate rewards are thought to have a stronger influence on decision making compared to delayed rewards, even when the delayed reward is more valuable (Appelhans et al., 2013). The tendency to discount the value of delayed rewards and accept the sooner rewards can lead to impulsive decision making through losing focus on long-term goals (Ainslie, 2001). 
The discounting of delayed rewards gets greater as the distance (time) between the two options increases (Bickel \& Marsch, 2001). Ainslie (1975) stated that delay discounting is a behavioural model of impulsivity, and within the lab, choice procedures may be the best way to empirically quantify it.

A number of factors have been associated with different levels of impulsivity. First, the literature suggests that children and adolescents have high levels of impulsivity, with impulsive tendencies decreasing with age (Green, Fry, \& Myerson, 1994; Green, Myerson, \& Ostaszewski, 1999; McCrae et al., 1999). Although, others suggest that whilst impulsive tendencies decrease in adulthood or middle age, they increase upon retirement (Read \& Read, 2004; Sozou \& Seymour, 2003). Second, it has been suggested that the income an individual receives is associated with the decrease in rates of delay discounting as someone ages (Green, Myerson, Lichtman, Rosen, \& Fry, 1996). For example, an individual on a good wage may discount to a greater extent as they do not have concerns over money, whereas someone on a less favourable wage may favour the lower values of money 'now' as the need for money is greater. Third, whilst impulsive tendencies occur across males and females, specific facets are more prevalent in one sex over the other (Feingold, 1994). For example, in their meta-analysis, Cross, Copping and Campbell (2011) found that males scored higher on risk taking and sensation seeing subscales, although results were similar to females in terms of delay discounting tasks.

\subsubsection{Impulsivity within eating behaviours}

Within clinical populations, a wealth of research evidence has demonstrated significant associations between impulsivity and the characteristics of disordered 
eating (Claes, Vandereycken, \& Vertommen, 2002, 2005; Davis et al., 2013; De Silva \& Eysenck, 1987; Fischer, Smith, \& Cyders, 2008; Galanti, Gluck, \& Geliebter, 2007; Toner, Garfinkel, \& Garner, 1987; Waxman, 2009). Claes et al. (2002) found that trait and state impulsivity scores were significantly higher in females with AN and BN compared to controls, but disappeared after controlling for cluster B personality disorders (American Psychiatric Association, 2013; e.g. antisocial and narcissist personality disorders). This is consistent with previous research finding a higher prevalence of impulsive behaviours among individuals with BED, BN and AN-purge subtype compared to healthy controls (Lowe \& Eldredge, 1993). It has been theorised that impulsivity is a moderator in the expression of eating disorders, and that individuals with a more impulsive nature are more likely to engage in binge/purge behaviours. Although, this theory has yet to be tested (Waxman, 2009). Greater levels of impulsivity have also been found in females with obesity compared to normal weight controls (Nederkoorn, Smulders, Havermans, Roefs, \& Jansen, 2006) and in individuals with BED compared with healthy controls (Nasser, Gluck, \& Geliebter, 2004). However, it should be noted that these differences were only found on behavioural, rather than self-report, measures of impulsivity.

Guerrieri, Nederkoorn, Stankiewicz, et al. (2007) suggest that three aspects of impulsivity could be related to eating behaviours, and in particular, the obesity epidemic (Davis, Levitan, Smith, Tweed, \& Curtis, 2006). The first is impulsiveness, defined as "acting on the spur of the moment without being aware of any risk involved" (Eysenck, Easting, \& Pearson, 1984). This may be related to eating behaviours as individuals may choose to give into their cravings rather than focusing on more important future goals such as losing weight. The second is response inhibition, which is when disturbed inhibitory control prevents an individual from being able to self- 
regulate and inhibit a response (Barkley, 1997). With regards to food, when an individual is faced with palatable food the adaptive response is to consume the food. However, in today's obesogenic environment this is unnecessary. The third is sensitivity to a reward, where individuals with high impulsivity detect and approach more rewarding stimuli (Avila, 2001). In terms of food, the sensitivity to reward may result in individuals consuming foods that are high in sugar and fat, as these have higher palatability than bland foods (Davis et al., 2007). In an experimental study, Guerrieri, Nederkoorn, Stankiewicz, et al. (2007) found that behavioural impulsivity significantly predicted total food intake, whereas self-reported impulsivity was only a marginally significant predictor of food intake in individuals with a healthy weight. The experiment primed impulsivity through a scrambled sentences task which subtly hinted at the concept of impulsivity versus a control condition with neutral sentences. They found no significant difference in food intake (consumption of milkshakes) between the experimental and control conditions. That said, during the experimental session participants felt like tasting the milkshake more. Therefore, it could be that impulsivity is related to a greater tendency to explore and try new foods.

Guerrieri, Nederkoorn, and Jansen (2007) noted that most research supporting a role of impulsivity in obesity has been conducted in clinical samples, arguing that such findings should be replicated in individuals with normal weight. To this end, they examined disordered eating thoughts and behaviours and food intake in high and low impulsive individuals. They found that self-reported high impulsive individuals consumed more food than those who reported themselves as being low impulsive. On average, the highly impulsive participants consumed an additional 27kcal. Although this difference is relatively small, Levitsky (2005) reported that a daily increase of 5$8 \mathrm{kcal}$ can lead to considerable weight gain in the long term. In contrast, when 
comparing high and low impulsive individuals on a behavioural task of impulsivity (stop-signal task; Logan et al., 1997), Guerrieri and colleagues (2007) found significant difference in food-intake between groups. Therefore, the relationship between impulsivity and food intake seems to differ depending on whether impulsivity is measured by self-report or via a behavioural measure. This helps to explain why findings concerning the relationship between impulsivity and eating behaviours are mixed.

Across the literature, delay discounting has also been associated with eating behaviours. For example, there is a greater preference for high energy-dense food 'now' for instant gratification versus healthier food choices later (Daniel, Stanton, \& Epstein, 2013), and this has been found in females with obesity (Appelhans et al., 2013). This preference may occur more frequently in individuals with obesity as they find food especially rewarding (Bonato \& Boland, 1983; Nederkoorn et al., 2006; Saelens \& Epstein, 1996). Being able to delay gratification for a reward may also be related to eating behaviours. As previously mentioned, delay or reward discounting involves the ability to delay a reward and can lead to an increased tendency to choose immediate small rewards over larger delayed ones (Ainslie, 1975; Monterosso \& Ainslie, 1999; Rachlin, 1995). In the context of emotional eating, if an individual is experiencing negative emotions, then those who can delay turning to food in favour of more adaptive strategies will benefit. Here, it is proposed that individuals concerned with the future, and particularly future health goals, are less likely to engage in emotional eating.

Due to the number of links between impulsivity and eating behaviour more broadly, it is not surprising that a connection can also be drawn between impulsivity and emotional eating. However, when focusing on the relationship between 
impulsivity and emotional eating specifically, the literature is somewhat mixed. Jasinska et al. (2012) found that inhibitory control was significantly associated with emotional eating in young adults with a healthy BMI, and that impulsivity was positively associated to overeating in response to negative affect. Consistent with this, Ebneter and colleagues (2012) found that impairments in impulsivity were associated with self-perceived greater emotional eating in restrained eaters. Interpreting this, it may be that an increase in food intake following negative affect is seen in restrained eaters because it impairs their usual control over their diet (Boon, Stroebe, Schut, \& Jansen, 1998).

In contrast, during an experimental study, Bekker et al. (2004) found no significant differences in self-perceived emotional eating between a high and low impulsive group following a mood manipulation task. Using the Barratt Impulsiveness Scale (BIS-11; Patton, Stanford, \& Barratt, 1995) to distinguish participants with high versus low impulsive tendencies, the authors assigned participants to a negative or neutral mood manipulation task. Results revealed no significant main effect of impulsivity for self-perceived emotional eating, but the impulsivity $\mathrm{x}$ negative affect interaction was significant. Individuals with high impulsive tendencies were more strongly influenced by the experience of negative affect, and in turn reported higher levels of self-perceived emotional eating.

Numerous studies have also explored the relationship between negative urgency and eating behaviour. First, Fischer et al. (2008) found significant relationships between negative urgency, (lack of) premeditation, sensation seeking and (lack of) perseverance and bulimic symptoms, although the relationship between negative urgency and bulimic symptoms had the largest effect size. In addition, negative urgency was significantly related to EDI-Bulimia in a clinical sample even 
after controlling for all other UPPS subscales (the urgency, premeditation, perseverance, sensation seeking scale; UPPS; Whiteside \& Lynam, 2001), anxiety sensitivity, anxiety, depression, the impulsive behaviours scale, positive affect and negative affect scale (PANAS; Watson, Clark, \& Tellegen, 1988) scores, and a range of demographic factors, (Anestis, Smith, Fink, \& Joiner, 2009). This suggests that a particularly strong relationship may exist between eating behaviour and negative urgency. Specifically, Anestis et al. (2009) stated that during times of low negative affect, individuals high in negative urgency may be able to control their behaviour. In contrast, during times of high negative affect, individuals high in negative urgency may need to acquire more adaptive problem-solving strategies. Similar findings have also been found within a student population (Anestis, Selby, \& Joiner, 2007). Here, Anestis and colleagues (2007) found that negative urgency significantly predicted EDI-Bulimia at time 1 and time 2 in a longitudinal study (3-4 weeks apart), even when controlling for depression, anxiety and the other subscales of the UPPS. Second, there is support for the relationship between negative urgency and emotional eating through shared genetic influences. Racine et al. (2013) asked female twins to complete a battery of self-reported measures exploring eating behaviours, negative urgency and negative affect, finding that negative urgency was significantly associated with dysregulated eating (i.e. emotional and binge eating). Negative urgency also significantly predicted emotional eating when controlling for negative affect. Moreover, there was a strong correlation between the genetic factors that influence negative urgency and the genetic factors that influence dysregulated eating behaviours. As a result, Racine and colleagues (2013) proposed that negative urgency likely increases the risk of developing binge and emotional eating through genetic mechanisms. 
In summary, impulsivity has been broadly associated with eating disorders and eating behaviour within the literature, although negative urgency is of particular interest for this thesis. Research conducted in both clinical and student populations have found strong associations between negative urgency and bulimia symptoms, overeating and emotional eating. The literature supports the theory that if an individual has an increased tendency to react rashly in response to negative affect, they may be more vulnerable to engaging in emotional eating. This suggests that impulsivity is a key factor to consider when exploring the mechanisms that may underpin emotional eating.

\subsubsection{Impulsivity and BMI}

Differences in levels of impulsivity have also been seen across BMI levels. In a study of adolescents, Delgado-Rico, Río-Valle, González-Jiménez, Campoy and Verdejo-García (2012) found that individuals with normal weight scored significantly higher on measures of sensitivity to reward and sensation seeking compared to individuals with 'excess weight' (the term adopted by the authors in the study to represent individuals with overweight and obesity). They also found that within individuals with 'excess weight', BMI was significantly related to negative urgency, positive urgency and a lack of perseverance. This suggests that for individuals with higher BMI scores, increased weight is associated with an increased tendency to act rashly in response to negative and positive affect and experience difficulties retaining focus on a goal. The authors also found that controlling for BMI in a regression model increased the predictive capacity of age and personality traits on negative and positive urgency. 
Within a student population with healthy BMI scores, Murphy, Stojek, and MacKillop (2014) also found that BMI was related to lack of premeditation. Indirect effects of impulsivity, and in particular the negative urgency and lack of perseverance subscales of the UPPS-P, on BMI were also found via food addiction. These findings may indicate that individuals who react without thinking to negative affect may turn to food to alleviate such sensations or are unable to maintain focus on challenging and regulating behaviours such as food addiction, resulting in a greater BMI score. Poor response inhibition has also been associated with weight gain during a one-year period, but only in individuals who report strong preferences for snack foods (Hofmann et al., 2014).

If individuals who are unable to delay gratification are more likely to consume tempting foods, it could be theorised that they may have a higher BMI as a result. However, research in support of this is mixed. On one hand, when comparing individuals with obesity and with a normal weight from a Dutch sample, Nederkoorn and colleagues (2006) found no significant differences in behaviour on a monetary delay discounting task. These findings were later replicated within a UK sample (Yeomans, Leitch, \& Mobini, 2008). Finally, Rasmussen, Lawyer, and Reilly (2010) studied monetary and food delay discounting tasks in a student sample. They found that individuals with high and low BMI (25\% and $75 \%$ quartiles) did not differ in their ability to delay discount in a food task. In contrast, a significant difference was found between high and low percentage body fat. On the other hand, significant differences in discounting behaviour have been observed between females, but not males, with a healthy weight and with obesity (Weller, Cook, Avsar, \& Cox, 2008). Furthermore, different delay discounting rates have been found between females with a healthy weight and those with obesity and BED (Davis, Patte, Curtis, \& Reid, 2010), with no 
significant difference between the obesity and BED groups. The differences also disappeared after one year of delay, suggesting that at a time beyond this (at two years); all groups discount the relatively small larger later reward $(\$ 100)$ at the same rate. The larger later reward was relatively small $(\$ 100)$ and time delays varied up to two years which may not accurately measure the rate of discounting.

\subsubsection{Impulsivity and emotional dysregulation}

Negative urgency is one of the key elements of impulsivity that influences maladaptive coping behaviours, of which evidence has been presented in the previous subsections. It has been shown to predict a number of behaviours including disordered eating in healthy individuals (Anestis, Selby, \& Joiner, 2007; Dir, Karyadi, \& Cyders, 2013). Difficulties in regulating emotions by acting rashly in response to negative affect can cause individuals to turn to food to help alleviate them. In an integrative review, Ferriter and Ray (2011) suggested that elevated levels of neuroticism and urgency likely cause an individual to experience greater negative emotions and to have difficulty regulating them. They further suggested that binge eating may occur in response to these situations in order to alleviate the negative sensations and enhance positive emotions, which reinforces the behaviour. Within female students, negative urgency has been found to pose the greatest risk to elevated binge eating (Emery, King, Fischer, \& Davis, 2013), constituting a significant main predictor of disordered eating alongside trait anxiety (Davis \& Fischer, 2013).

Robinson, Kosmerly, Mansfield-Green, and Lafrance (2014) explored the relationships between gender and BMI in disordered eating in healthy undergraduate students. In their study, females reported weaker emotional regulation skills and were 
less able to use adaptive strategies to regulate their emotions. Although, the authors suggested that this may be due to females being more likely to admit to experiencing such difficulties and their tendency to experience emotions more intensely. Overall, they found that gender, BMI and difficulties regulating emotions were related to disordered eating behaviours and attitudes. Irrespective of gender, they also found that impulse control difficulties coupled with a higher BMI was associated with a greater number of disordered eating behaviours. Therefore, emotional eating (an emotional regulation behaviour related to impulse control) may be related to BMI, highlighting that poor impulse control following a reduced ability to regulate emotions may be linked to emotional eating and BMI.

\subsubsection{Impulsivity and alexithymia}

Behaviours and disorders, such as alcohol use disorder, self-injury and binge eating, associated with impulsivity have also been related to alexithymia (Carano et al., 2006; Shishido et al., 2013; Wheeler, Greiner, \& Boulton, 2005; Zlotnick et al., 1996). However, only a handful of studies have explored the associations between alexithymia and impulsivity explicitly. One study showed that individuals with alexithymia were more likely to act impulsively when experiencing positive and negative affect; engaging in more drinking behaviours as a result (Shishido et al., 2013). Shishido and colleagues (2013) proposed that deficits in the cognitive representation of emotions, namely a lack of understanding and comprehension, may lead to impulsive action when experiencing heightened emotional arousal. Furthermore, when exploring the associations between alexithymia and personality traits in undergraduate students, small significant positive correlations were found 
between impulsiveness as measured by the NEO Personality Inventory Revised (NEOPI-R; Costa \& McCrae, 1992) and total and DIF scores of the TAS-20 (Zimmermann, Rossier, De Stadelhofen, \& Gaillard, 2005). The DIF subscale was also significantly correlated to the I7 measure of impulsivity (Impulsiveness Questionnaire; Eysenck, Pearson, Easting, \& Allsopp, 1985) but total alexithymia scores were not. This highlights how the relationship between impulsivity and alexithymia may differ depending on the measure, and facet, of impulsivity examined. Overall, this suggests that the relationship between alexithymia and impulsivity may be influenced by other factors and that associations are only with specific aspects of impulsivity.

Investigating the role of impulsivity and alexithymia in deregulatory behaviour more broadly, Fink, Anestis, Selby, and Joiner, (2010) observed associations between alexithymia and urgency, lack of premeditation and lack of perseverance. A mediation analysis identified urgency as fully, and lack of premeditation as partially, meditating the role between alexithymia and dysregulated behaviours. The dysregulated behaviours explored were all deemed as possible behaviours used to regulate emotion, including taking drugs, consuming food and rumination. The authors concluded that if individuals are unable to recognise and process their emotions adaptively, then they may find this confusing and therefore, act rashly in response by engaging in dysregulated behaviours.

\subsubsection{Interim Summary}

In summary, impulsivity has been broadly associated with eating disorders and behaviour, although results tend to differ depending on how impulsivity is conceptualised and measured. An important consideration is that self-report 
questionnaires and behavioural impulsivity tasks do not correlate well with one another, possibly as different aspects of impulsivity are being assessed (Guerrieri, Nederkoorn, Stankiewicz, et al., 2007). Individuals who are impulsive and steeply discount as a result, are less likely to think about their best interests in the long term (Manwaring, Green, Myerson, Strube, \& Wilfley, 2011). Therefore, impulsivity may play a role in emotional eating as when an individual experiences negative affect they may turn to food to alleviate these sensations instead of focusing on any long term plans such as healthy eating or dieting, or to target the cause of the emotion. Of particular interest to this thesis is the role of negative urgency. Research conducted in both clinical samples and student populations have found strong associations between negative urgency and bulimia symptoms, overeating and emotional eating. The proposed link between the negative urgency facet of impulsivity and emotional eating is that individuals act impulsively when experiencing distress or upsetting emotions, acting rashly in response to alleviate the sensations. Literature supports the theory that an individual's ability to not act rashly in response to negative affect is a key consideration in the mechanisms that may underpin emotional eating. Following this theory, it is logical to also explore the role of negative affect. The basic premise is that individuals high in negative urgency may only experience difficulties resisting acting rashly when combined with high levels of negative affect. Negative affect is also an overlapping feature of emotional dysregulation and alexithymia.

\subsection{Negative Affect}

Emotional eating is grounded in the idea that the consumption of food follows an emotional experience (van Strien et al., 1986). Therefore, it seems logical that an 
individual's level of negative affect may play a role. Negative affect for the purpose of this thesis is split into anxiety and depression. At some point all individuals experience low mood but for some it is pervasive, causing emotional, physiological and cognitive problems referred to as depression (Bennett, 2015; Bennett, 2001). The DSM-5 definition of depression includes symptoms of diminished interest, fatigue, feelings of worthlessness and a depressed mood for most of the day (American Psychiatric Association, 2013). Over time, individuals experience situations in which they are worried or nervous, but for some individuals the worries are uncontrollable and occur almost daily for a prolonged period of time (American Psychiatric Association, 2013). Although clinical levels of depression and anxiety are not explored in this thesis, the role of negative affect along a continuum is important to consider when exploring emotional eating. The prevalence of depression is greater in females (Piccinelli \& Wilkinson, 2000) and this gender difference has been reported in samples from multiple countries (van de Velde, Bracke, \& Levecque, 2010). Females are also more likely to develop anxiety disorders (McLean \& Anderson, 2009). However, as few longitudinal studies have been conducted, it is difficult to distinguish differences in depression and anxiety across the lifespan. Even so, available literature suggests that as individuals age they are less susceptible to anxiety and depression, although this pattern was not consistent across all studies in a review by (Jorm, 2000).

\subsubsection{Negative affect in eating behaviours}

Negative emotions are often seen as predispositions to bulimic behaviours (Stice, 2002) and a number of studies have found significant associations between depression and eating disorders (Bydlowski et al., 2005; Gilboa-Schechtman, Avnon, 
Zubery, \& Jeczmien, 2006; Kucharska-Pietura, Nikolaou, Masiak, \& Treasure, 2004). Specifically, it has been suggested that prior to a binge eating episode, individuals with $\mathrm{BN}$ report experiencing increased negative mood (Davis, Freeman, \& Garner, 1988). However, it has also been proposed that bulimic symptoms may lead to increased negative affect and the start of a vicious cycle where negative affect may become the consequence and not the trigger (Elmore \& de Castro, 1990; Stice, 1998).

Within a broad sample of males and females, anxiety and stress were found to account for a greater variance in binge eating symptoms compared to depression (Rosenbaum \& White, 2015). In further support, Freeman and Gill (2004) found that elevated levels of stress were associated with discrete episodes of binge eating even after controlling for levels of depression in a female sample who self-identified as having BED. They used diaries to gather information on daily stresses and compared this to the self-reported incidences of binge eating. In contrast, emotional eating was significantly associated to anxiety, tension, stress and worrying in adolescent females and a confused mood in males (Nguyen-Rodriguez et al., 2009). Nguyen-Rodriguez and colleagues (2009) also expected to find an association with depression but the results were not significant. They proposed that anxiety may be more common during adolescence, with depression more common in adults. Goossens, Braet, van Vlierberghe and Mels (2009) found significant associations between emotional eating and both anxiety and depression. The authors found that emotional eating mediated the relationship between anxiety and loss of control eating with trend significance. Associations between emotional eating and depression were also found in the MooDFood prevention study (Paans et al., 2018). Paans and colleagues (2018) found that sub-syndrome and major depressive disorder were associated with increase severity of emotional eating with findings comparable across four European countries 
(UK, Spain, Germany and the Netherlands). This indicates that both clinical and nonclinical levels of depression are associated with emotional eating and it is an important factor to consider.

Studies within non-clinical populations commonly use mood induction procedures, in which negative and positive feelings are manipulated, to explore the role of negative affect on emotional eating (e.g. Bongers et al., 2013; Macht, Roth, \& Ellgring, 2002; Udo et al., 2013). For example, in a sample of females with obesity, Chua and colleagues (2004) found that participants consumed more chocolate after watching a negative versus positive film excerpt. Schneider et al. (2011) found trait anxiety to be associated with food intake in the laboratory following an anxiety mood induction compared to a neutral mood induction. However, this effect was only observed in participants with obesity. They suggested that trait anxiety may play a role in the vulnerability of individuals in emotional eating and is linked with avoidance motivation. Similar findings were found by Jansen, Vanreyten, and Balveren (2008), whereby following a high negative mood induction, participants with overweight and obesity, who also had high levels of negative affect (mean Beck depression inventory $(\mathrm{BDI})=17 ; \mathrm{mild} /$ moderate depression $)$, consumed more in comparison to individuals with overweight and obesity with low negative affect, and individuals with a healthy weight. Other studies have also found an increase in consumption following a negative mood in comparison to control and/or positive moods (e.g. Fay \& Finlayson, 2011; Scattolon \& Nicki, 1995; Wallis \& Hetherington, 2004). However, negative affect has not been consistently found to result in increased consumption. Within female students with a healthy weight, a lower desire to eat was found in participants in the negative mood induction condition compared to the those in the neutral condition (Loxton, Dawe, \& Cahill, 2011). Bongers et al. (2013), Evers et al. (2009) and Wallis and 
Hetherington (2009) are also examples of studies which have found lower intake or no significant difference in intake following a negative mood induction. A more in-depth review of mood induction and food intake literature is in Chapter Four.

Some authors suggest that individual aspects of negative affect play a unique role in binge eating (Haedt-Matt \& Keel, 2011; Rosenbaum \& White, 2015), and specifically, emotional eating (Goossens et al., 2009). First, it is proposed that engaging in binge eating could decrease levels of anxiety but increase levels of depression (Haedt-Matt \& Keel, 2011; Rosenbaum \& White, 2015) and second, it is thought that emotionally eating following anxiety is a coping mechanism to alleviate hyper-arousal and following depression it is to increase more positive emotions (Goossens et al., 2009). Together, these theories suggest that depression and anxiety have a unique role to play in emotional eating and that they should be considered as two distinct emotional states.

\subsubsection{Negative affect and BMI}

Depression and obesity have been linked reciprocally in meta-analyses of longitudinal studies. Luppino et al. (2010) found that individuals with obesity were at increased risk of depression, and that depression was predictive of future weight gain and obesity. In a sample of adults with overweight and obesity, higher levels of emotional eating in response to depressive feelings, anxiety or feelings of anger were significantly associated with greater BMI scores (Braden, Musher-Eizenman, Watford, \& Emley, 2018). Furthermore, Braden et al. (2018) found that eating in response to depression, anxiety, or boredom was associated with greater disordered eating and emotion regulation difficulties, with the latter associated with poorer psychological 
well-being. This is suggestive of a possible vicious cycle. Poor emotional regulation may cause an increase in negative affect where individuals respond by emotionally eating in response. This reinforces the view that emotional eating is an emotional regulation strategy which is detrimental to mental health. In addition, Braden and colleagues (2018) found that all aspects of emotional regulation (as measured by the Difficulties in Emotional Regulation Scale; DERS; Gratz \& Roemer, 2004), except for lack of emotional awareness, were significantly predicted by emotional eating in response to depressive symptoms.

Further links between depression, obesity and emotional eating have also been found in the literature (Lazarevich, Irigoyen Camacho, Velázquez-Alva, \& Zepeda Zepeda, 2016; van Strien, Konttinen, Homberg, Engels, \& Winkens, 2016). Lazarevich and colleagues (2016) conducted a large-scale questionnaire study on 1453 male and female students with a wide range of BMI scores. They found significant positive associations between depression, emotional eating and BMI for both males and females. In addition, they found a direct effect of depressive symptoms on BMI and an indirect effect via emotional eating scores. The indirect effect accounted for $23.1 \%$ of the total effect in males and $25 \%$ in females. That is, students who experienced higher levels of depressive symptoms and reported a greater tendency to emotionally eat, had higher BMI scores. In support, van Strien et al. (2016) also found that emotional eating mediated the relationship between depression and future body weight gain in females, but not males. External eating or restraint did not mediate the relationship between depression and weight gain in either sex.

In addition to depression, several studies have also found a linear relationship between anxiety and BMI (Jorm et al., 2003; Zhao et al., 2009), whereas an inverted U-shape was found by Haghighi et al. (2016). Specifically, Zhao et al. (2009) found 
males with class III obesity were $42 \%$ more likely to experience lifetime diagnosed anxiety, and females with overweight or obesity (class I, II, or III) were 9-17\% more likely to have clinically diagnosed anxiety at some point in their lives. They also found significant associations between depression and BMI (e.g. individuals with class III obesity were $38 \%$ more likely to currently have depression and $40 \%$ more likely to have received a lifetime diagnosis of depression). Furthermore, Jorm et al. (2003) found that obesity was associated with higher levels of anxiety and depression but only in females. In contrast, Haghighi et al. (2016) found that lower anxiety scores were related to both low and high BMI scores, with high anxiety scores related to medium to high BMI scores in outpatients with anxiety disorders. Differences in findings may be due to the assessment of anxiety. For instance, Haghighi et al. (2016) assessed current anxiety levels whereas Zhao et al., (2009) assessed lifetime anxiety. Haghighi and colleagues (2016) also used a sample of patients with diagnosed affective disorders whereas Zhao et al. (2009) and Jorm et al. (2003) examined the relationship between anxiety, depression and BMI in large representative samples.

\subsubsection{Negative affect and emotional dysregulation}

In both a community sample and a group of individuals with eating disorders, negative affect was associated with emotion-oriented coping (Spoor et al., 2007). Emotion-oriented coping is an emotion regulation style that aims to alleviate negative emotions by managing stressful situations or the emotions generated from such situations (Endler \& Parker, 1994). Negative affect was also significantly related to higher levels of avoidance distraction (Spoor et al., 2007), a strategy associated with avoiding stressful situations by distracting oneself (Endler \& Parker, 1994). Emotion 
oriented coping and avoidance distraction were strongly related to greater levels of emotional eating, and when controlling for these strategies, negative affect was not significantly related to emotional eating (Spoor et al., 2007). This suggests that negative affect does not have a unique relationship with emotional eating above coping strategies such as emotion-oriented coping and avoidance distraction.

\subsubsection{Negative affect and alexithymia}

Exploring emotions in general, Zeeck et al. (2011) found that positive emotions were significantly and negatively associated with total alexithymia scores, with the opposite found for negative emotions. Consistent with this, greater levels of depression and anxiety have been associated with alexithymia (Honkalampi et al., 2000). In particular, one study found that $62.3 \%$ of males and $72 \%$ of females with alexithymia had some level of depression in comparison to only $12.9 \%$ and $18.6 \%$ in males and females without alexithymia respectively (Honkalampi et al., 2000). In addition, Parker and Taylor (1997) found that alexithymia and depression were highly correlated but distinct constructs.

Within depression, alexithymia has been described as a coping defence mechanism secondary to depression (Rief, Heuser, \& Fichter, 1996) whilst others argue it is a separate personality trait (Freyberger, 1977; Wise, Mann, Mitchell, Hryvniak, \& Hill, 1990). Even though alexithymia is associated with reduced awareness of emotions, it is argued that emotional awareness is not completely absent. Therefore, individuals with alexithymia can still express sensations of depression and anxiety (Hendryx, Haviland, \& Shaw, 1991). This highlights that a vulnerability to negative emotions may derive from a reduced ability to think about positive past 
events, and therefore, individuals are unable to benefit from the protection of positive thoughts (Luminet, Zech, Rimé, \& Wagner, 1998; Morrow \& Nolen-Hoeksema, 1990; Prince \& Berenbaum, 1993). It has also been suggested that individuals who have an emotional processing deficit, such as alexithymia, and experience negative affect have poor social support networks and are unable to benefit from having others around (Lumley, Ovies, Stettner, Wehmer, \& Lakey, 1996). This could further exacerbate their tendency to engage in disordered eating and experience greater levels of psychopathology (Jackson, Weiss, Lunquist, \& Soderlind, 2005; Keltner \& Kring, 1998; Marroquín, 2011; Ridout et al., 2010). Furthermore, the presence of reduced motivation, energy and a negative self-concept may result in individuals exaggerating levels of alexithymia (i.e. bias towards negative perceptions of ability; Lane et al., 1998). This indicates that the association between alexithymia and depression works both ways; a vulnerability to negative affect which causes a reduction in the ability to comprehend emotions. Taylor et al. (1997) stated that the affect regulation processes that individuals with alexithymia often engage in are the mechanisms that drive the physical and mental health problems that are comorbid with alexithymia. For example, lack of appropriate emotion expression or suppressing emotions. Finally, greater levels of depression are often reported in female samples, suggesting that depression may influence the strength of association between sex and alexithymia. Larsen et al. (2006) found that alexithymia was more strongly related to sex after controlling for levels of depression. This suggests that sex is an important factor to consider when exploring the relationship between alexithymia and depression.

Evidence is also available for the associations between anxiety and alexithymia. Using the TAS-26 (Taylor et al., 1985), significant associations were found between state anxiety, DIF and reduced daydreaming, trait anxiety, DIF and 
DDF, and between reduced daydreaming, depression and DIF and DDF (Berthoz, Consoli, Perez-Diaz, \& Jouvent, 1999). Berthoz et al. (1999) found that total alexithymia scores and DIF remained significantly correlated with trait and state anxiety after controlling for depression. Furthermore, when controlling for anxiety, depression was no longer significantly related to alexithymia. In support, Karukivi, Hautala, Kaleva, et al. (2010) found that alexithymia was significantly associated with anxiety in older adolescents and the associations remained significant when controlling for depression and alcohol consumption. In sum, there is clear support for the associations between alexithymia and anxiety.

Despite being highly correlated, alexithymia and depression remain distinct constructs. Marchesi et al. (2000) conducted a factor analysis of the TAS-20 and the Hospital Anxiety and Depression Scale (HADS; Zigmond \& Snaith, 1983). They concluded that depression is a different construct to alexithymia, but that there are some overlapping features across anxiety and DIF. In addition, depression was a unique predictor of alexithymia (Luminet, Bagby, Wagner, Taylor, \& Parker, 1999), further supporting the associations between alexithymia and depression. In contrast, when exploring the prevalence of alexithymia in individuals with $\mathrm{AN}$ and $\mathrm{BN}$, controlling for depression and anxiety resulted in no differences in alexithymia ratings across groups (Eizaguirre et al., 2004). This highlights that alexithymia is more greatly related to affect than eating disorders. The authors concluded that alexithymia may present comorbidly with eating disorders and alexithymia, but in some cases, it may present as a secondary condition to anxiety and/or depression.

Within clinical eating disorders, significantly greater levels of alexithymia and depression have been found in individuals with AN in comparison to individuals with BN and healthy controls (Corcos et al., 2000). When Corcos et al. (2000) controlled 
for levels of depression, the difference in alexithymia scores between individuals with $\mathrm{AN}$ and $\mathrm{BN}$ was no longer significant. They concluded that increased rates of $\mathrm{AN}$ were more closely linked to depression and alexithymia in comparison to BN. In support, Torres and colleagues (2015) found that depression mediated the relationship between total alexithymia scores (partially), DIF (partially) and DDF (fully) with AN. Finally, within a non-clinical sample, Ridout et al. (2011) found that depression and alexithymia accounted for $53 \%$ of variance in total EDI scores, providing support for the notion that depression and alexithymia individually and combined, predicted disordered eating.

\subsubsection{Negative affect and impulsivity}

The majority of research surrounding negative affect and impulsivity is centred on individuals experiencing intense emotions and acting in a way to alleviate them. The immediate reactions to emotion are often rash and not thought through (Tice, Bratslavsky, \& Baumeister, 2001). Support for these associations can be found through biological mechanisms; the serotonin pathways involved in emotional experiences can inhibit dopamine pathways which lead to reductions in behavioural inhibitions in response to emotions (Patterson \& Newman, 1993).

Additional support is also available through eating behaviour research. In a study exploring the relationships between negative urgency, negative affect and disordered eating, Davis-Becker, Peterson, and Fischer (2014) found that negative urgency was robustly related to all negative mood states (fear, hostility, guilt and

sadness; PANAS-X; Watson \& Clark, 1999). This indicates that individuals with high levels of negative urgency experience a different and unique emotional experience to 
those with low levels of negative urgency. However, they found that negative urgency did not moderate the relationship between negative affect and disordered eating. Focusing on depression, Cyders and Coskunpinar (2011a) found that negative urgency significantly moderated the relationship between depression and health-related disability (extent to which health condition limit life activities for an individual; Bruce, Seeman, Merrill, \& Blazer, 1994). They found that as levels of negative urgency increased, the relationship between maladaptive coping behaviours, such as smoking, alcohol consumption and binge eating, also increased. They concluded that maladaptive coping and negative urgency may lead some individuals with depression to develop health-related disability when others do not. In a previous study, Cyders and Coskunpinar (2010) found that negative urgency significantly moderated the relationship between depression and alcohol use. As it is proposed that emotional eating and alcohol consumption are classed as coping behaviours, it is possible that the associations found between negative urgency and alcohol could translate to emotional eating.

Trait anxiety has been linked to increased global eating pathology alongside negative urgency (Davis \& Fischer, 2013). Negative urgency also significantly moderated the relationship between anxiety and physiological dependence symptoms of alcohol (Menary et al., 2015). The associations between anxiety and physiological dependence symptoms became stronger as levels of negative urgency increased. Menary and colleagues (2015) also found that indirect effects of anxiety and negative urgency on alcohol related consequences through coping strategies. They suggest that the effects of anxiety and negative urgency are additive rather than overlapping, although longitudinal studies are needed to confirm this. In combination, these studies suggest that there is a strong relationship between negative urgency and negative 
affect, which in turn, may result in maladaptive behaviours. In turn, support is provided for the inclusion of negative affect alongside negative urgency in exploring the role they may play in emotional eating.

\subsubsection{Interim Summary}

Overall, greater levels of negative affect and clinical levels of depression and anxiety have been associated with emotional eating. In addition, evidence suggests that negative affect is related to alexithymia and impulsivity, with further evidence highlighting that higher levels of anxiety and depression are associated with increased weight gain and BMI. Therefore, negative affect is a further factor which is important to consider when exploring the mechanisms underpinning emotional eating. Here, negative affect is referring to general levels of depression and anxiety experienced by individuals and not specific depressing or anxiety provoking events. It is thought that an individual's predisposition to experiencing depression and anxiety will influence how an individual reacts to the experience of negative affect.

\subsection{Theoretical Framework}

The current literature suggests that emotional eating may be influenced by a number of factors, such as alexithymia, impulsivity and negative affect. These factors have been shown to be related to one another and can be encompassed under the term of emotional dysregulation. However, relatively few studies have attempted to explore the relationship between alexithymia, impulsivity, affect and emotional eating inclusively. 
Pike (2013) asked female students to complete the TAS-20, DEBQ, UPPS and the Depression, Anxiety and Stress Scale (DASS-21; Lovibond \& Lovibond, 1995). Alexithymia and the TAS-20 subscales were significantly and positively related to emotional eating, negative urgency and lack of perseverance. Emotional eating was significantly and positively related to negative urgency and lack of perseverance, and significantly negatively related to sensation seeking. In addition, Pike (2013) explored the factors related to BMI, finding weak positive significant associations between total, DIF and DDF alexithymia scores, negative urgency and lack of perseverance. Depression and anxiety were also significantly associated to BMI. Within a moderation analysis, negative urgency did not moderate the relationship between alexithymia and emotional eating. Alexithymia and depression accounted for $25 \%$ of the variance in emotional eating, but anxiety was not a significant predictor. These findings suggest that the relationship between alexithymia and emotional eating is complex. Furthermore, Pike (2013) suggested that although negative urgency did not moderate the relationship it is likely to be key factor worthy of further investigation.

In a similar study, Fink et al. (2010) found that alexithymia scores significantly predicted the Behavioural/Interpersonal Emotional Regulation Scale (BIER; unpublished); which contains an emotional eating scale. Negative urgency also predicted BIER and fully mediated the pathway between alexithymia and maladaptive outcomes, such as emotional eating (as measured by the BIER). To test for directionality, the reverse model was explored. Results were not significant, suggesting that negative urgency explained the relationship. When the authors explored the other factors of the UPPS-P, (a lack of) premeditation partially mediated the relationship between alexithymia total scores and BIER. This study provides a 
good theoretical basis from which to explore individual dysregulated behaviours such as emotional eating.

Ouwens et al. (2009) proposed that the relationship between depression and emotional eating was mediated by impulsivity and the DIF facet of alexithymia. Within an all-female sample, positive significant correlations were found between DIF, impulsivity (EDI-2; Garner, 1991) and emotional eating (DEBQ). Their model found that depression was positively and directly associated with emotional eating and there was also an indirect effect via DIF and impulsivity. They tested the direct and indirect pathways with external eating (DEBQ), finding no significant results for depression and DIF, but a significant association between impulsivity and external eating. This model highlights the role emotional dysregulation may play in emotional eating.

The three studies presented here support the current theory that poor emotional regulation may influence emotional eating through difficulties comprehending emotions, heightened levels of affect and poor impulse control. Although Ouwens and colleagues (2009) have attempted to investigate a more inclusive model, they neglected to explore the alexithymia construct as a whole as well as its three core features. In addition, the proposed model in this thesis aims to examine negative affect in terms of both depression and anxiety and to explore impulsivity through the specific facet of negative urgency.

In sum, literature suggests alexithymia, negative affect and impulsivity may increase one's susceptibility to emotional eating. It is proposed that the associations between alexithymia, impulsivity, and negative affect constitute important mechanisms underpinning emotional eating. Individuals who experience greater difficulty understanding their emotions (alexithymia) may be more vulnerable to 
experiencing increased levels of negative affect. As a result, they may react rashly and without thinking to avoid the sensations of negative affect (negative urgency) and in turn engage in emotional eating as a coping mechanism (see Figure 1). Following the consumption of food to alleviate the sensations of negative affect, individuals may then feel guilty and this in turn could lead to the cycle commencing once more (see Figure 2).

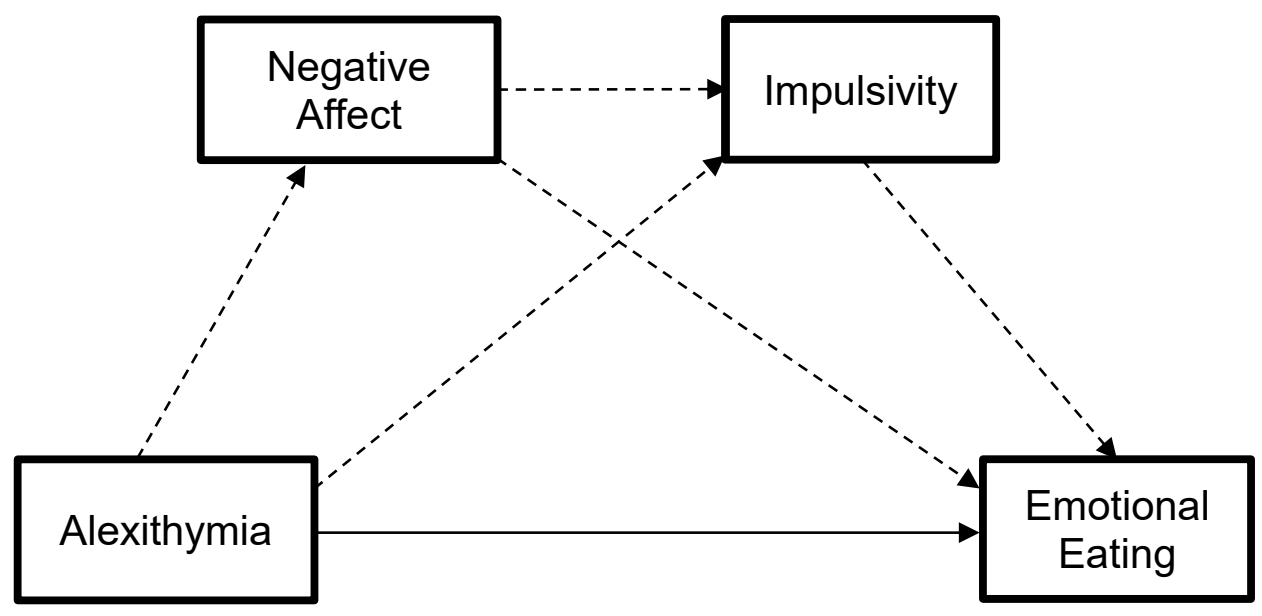

Figure 1. Inclusive model of emotional eating: The proposed direct and indirect relationships between alexithymia and emotional eating, including negative affect and impulsivity as mediators. 


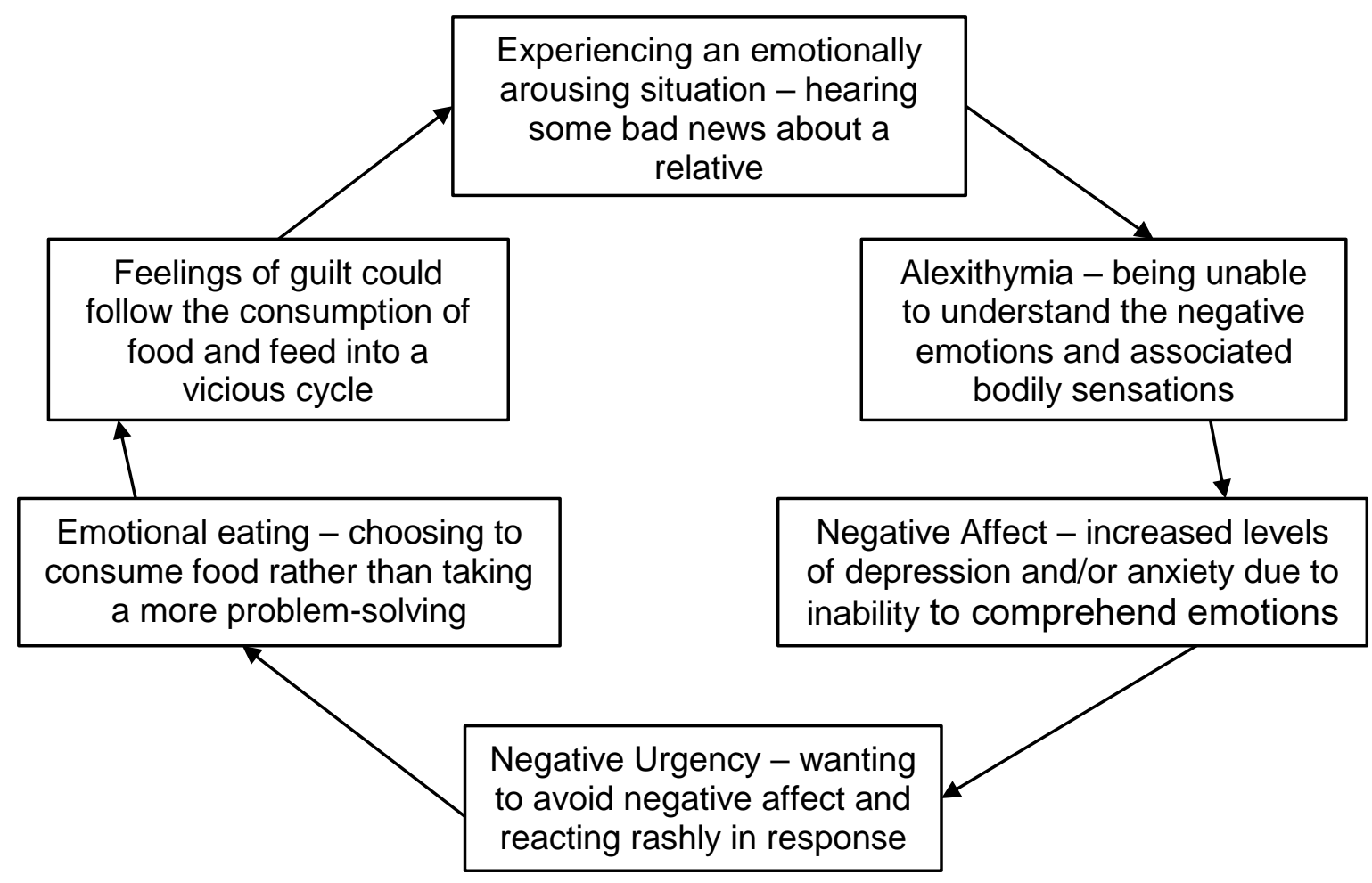

Figure 2. Example situation of a cycle of emotional eating.

\subsection{Overall aims}

The overarching aim of this thesis is to elucidate the mechanisms that underpin emotional eating. Specifically, it aims to explore the role of emotional dysregulation, as conceptualised by alexithymia, impulsivity (negative urgency and delay discounting) and negative affect (defined as either depression or anxiety), in understanding emotional eating. Previous literature has linked these constructs together individually and has also begun to explore their interactions. However, the current research is the first to explore a theoretical model of emotional eating inclusive of all factors. This thesis presents five studies that aim to develop a novel model of emotional eating. 
This thesis aims to:

1. Use alexithymia as a correlate of emotional dysregulation to explore its role in emotional eating.

2. Examine the key contributing factors that influence the relationship between emotional dysregulation and emotional eating, and in turn BMI.

3. Test the theoretical model of emotional eating, as presented in Figure 1.

4. Investigate whether simulating emotional dysregulation in an experimental setting directly affects food intake.

5. Extend the theoretical model to account for interoceptive awareness and feeling fat. 


\section{Chapter Two: The relationship between Alexithymia, Negative Affect, Impulsivity, Emotional Eating and BMI}

\subsection{Introduction}

The literature review presented in Chapter One highlighted that several factors may influence emotional eating and obesity, including alexithymia, impulsivity and levels of negative affect. However, previous research examining the specific relationship between alexithymia, emotional eating and obesity has produced mixed findings, raising the possibility that the relationship may be mediated by multiple unknown factors. Consistent with this line of thinking, Ouwens et al. (2009) found that DIF, impulsivity and depression were all significantly correlated with emotional eating, and that depression was directly and indirectly (through DIF and impulsivity) related to emotional eating. To expand this initial work, the novel theoretical model first presented in Chapter One seeks to further explore the mechanisms underpinning emotional eating by: (a) exploring negative urgency as a more specific facet of impulsivity; (b) examining negative affect in terms of both depression and anxiety; (c) fully exploring the alexithymia concept as a whole as well as its different facets, and (d) examining alexithymia, emotional eating, negative urgency, and negative affect in one inclusive model. To test the proposed theoretical model in a robust manner, this chapter will present the results of an exploratory analysis in a student sample (Study One), followed by a self-replication to test the applicability of the model in a more representative general population sample (Study Two). 


\subsection{Study One: Aims}

The overarching goal of Study One was to comprehensively explore the potential role of emotional dysregulation in emotional eating. The first aim was to explore the associations between emotional eating and alexithymia, impulsivity, negative affect, and BMI. Impulsivity was explored via a self-report measure (UPPSP; negative urgency) and a behavioural measure (delay discounting). Significant associations between emotional eating, negative affect, alexithymia, impulsivity, and BMI were predicted. In addition, significant associations were expected to occur between alexithymia, negative affect and impulsivity.

The second aim was to comprehensively test the proposed theoretical model, incorporating negative affect (anxiety and depression separately) and impulsivity (negative urgency) as possible mediating factors in the relationship between alexithymia and emotional eating (see Figure 1). The model proposed that individuals who have difficulty understanding their emotions (alexithymia) are vulnerable to experiencing increased levels of anxiety and depression (negative affect), which may cause them to act rashly in response (negative urgency) and engage in emotional eating.

In addition, as emotional eating may increase BMI in the long-term (Koenders \& van Strien, 2011; Sung et al., 2009; van Strien, Herman, et al., 2012), the final aim of the study was to expand the theoretical model to explain variance in BMI (see Figure $3)$. 


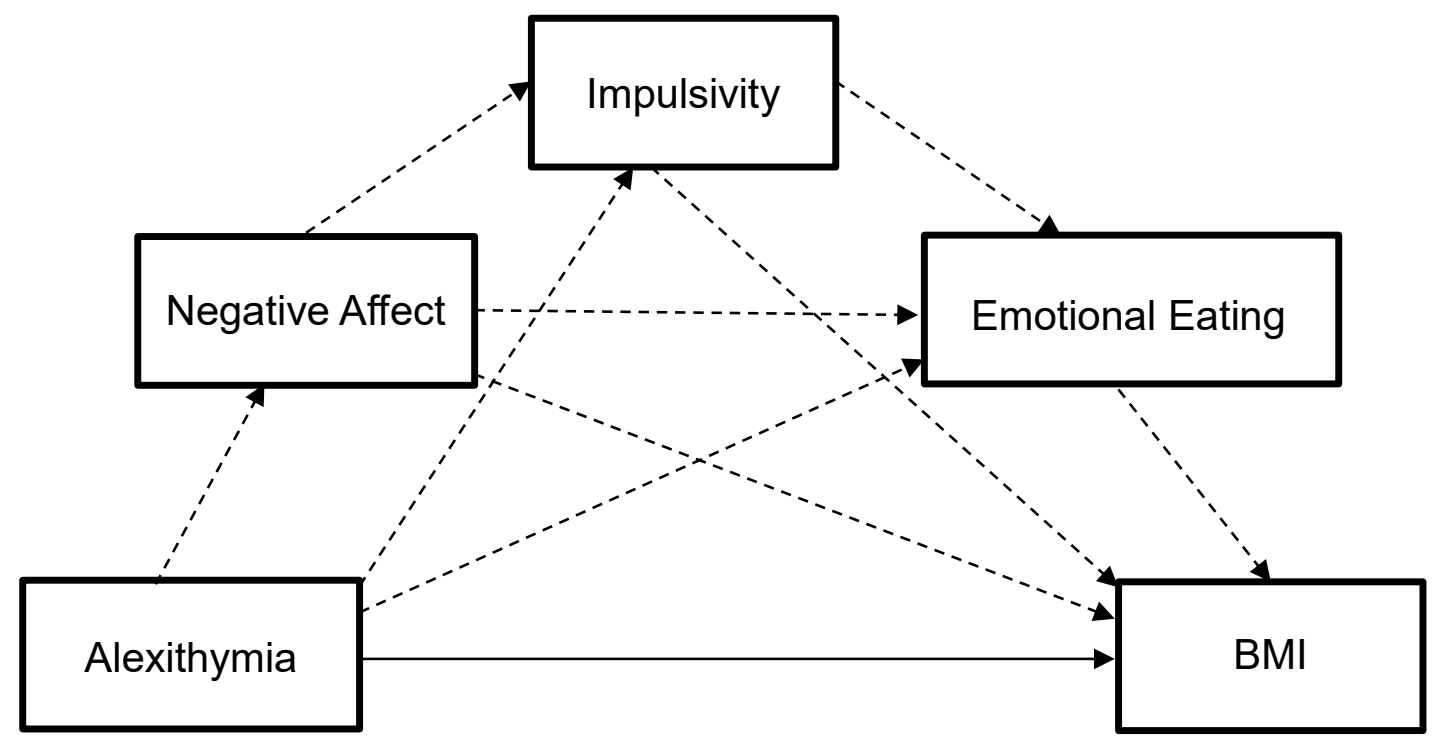

Figure 3. Inclusive model of BMI: The proposed direct and indirect relationships between alexithymia and BMI including negative affect, impulsivity and emotional eating as mediators.

\subsection{Study One: Methods}

\subsubsection{Participants}

To be eligible to take part in the research, participants had to report no history of eating, addictive or substance use disorders. Participants also had to be $18-65$ years of age; exclusion either side of this age bracket was due to possible involvement of the frontal lobes in the development of alexithymia and associated emotional deficits (e.g. under development or deterioration of the frontal lobes across the lifespan which may affect emotional processing and regulation; Wood \& Williams, 2007). 130 students from Swansea University initially met these criteria. However, five were subsequently removed owing to a reported history of eating disorders (disclosed after completion) or missing height and weight data which meant BMI could not be calculated. 
Of the remaining 125 participants, $107(85.60 \%)$ were female and the mean age of the sample was 20.85 years of age $(S D=3.06$, range $=18.27-36.87$ years $)$. $115(92.00 \%)$ participants were single, with the remaining $10(8.00 \%)$ co-habiting. The vast majority of participants reported their ethnicity as White, $90.40 \%(n=113$, with relatively few reporting their ethnicity as Asian $(n=3,2.4 \%)$, Black $(n=2,1.6 \%)$, Mixed $(n=6,4.8 \%)$ or other $(n=1, .8 \%)$. Mean BMI was $23.99(S D=4.34)$ with values ranging from 16.84 to $41.21 \mathrm{~kg} / \mathrm{m}^{2}$. Weekly alcohol consumption ranged from 0-40 units $(M=7.74, S D=7.29)$ and $13(10.4 \%)$ reported themselves as regular smokers. All participants received course credit in exchange for their participation.

An a priori power analysis (G*Power; Faul, Erdfelder, Lang, \& Buchner, 2007) indicated that a minimum sample size of 84 participants was required (effect size $=.25$ - medium; power $=.8)$. Therefore, the final sample size $(N=125)$ exceeded the number of participants required to have sufficient statistical power.

\subsubsection{Measures}

\subsubsection{Demographics}

Participants provided their date of birth, sex, marital/relationship status and ethnicity. Participants were also asked if they had a history of eating, addictive or substance use disorders.

\subsubsection{Positive Affect and Negative Affect Schedule}

(PANAS; Watson, Clark, \& Tellegen, 1988). The PANAS consists of 20 affect words to measure positive (10-items) and negative (10-items) affect during the past week. Defined by Watson et al. (1988), positive affect (PA) reflects the extent to which a person feels enthusiastic, excited, attentive, and alert. High PA is a state of high 
energy, full attention, and pleasurable engagement, whereas low PA is characterised by sadness, low arousal and lethargy. In contrast, negative affect (NA) involves feelings of distress and aversive mood states, with high NA associated with anger, guilt, nervousness, hostility, and irritability, and low NA associated with calmness and composure. Participants respond by choosing the answer that best describes them on a five-point scale from 1 ("very slightly or not at all") to 5 ("extremely"). There is no reverse coding and subscales scores are calculated by summing responses to the PA and NA items respectively. Subscale scores range from 20-50, with higher scores equating to a greater level of PA and NA. In line with previous research, high levels of internal consistency were found in the current study (PA: $\alpha=.88$, NA: $\alpha=.87$; Watson et al., 1988). The PANAS has been shown to be a reliable and valid measure in non-clinical and general population samples (Crawford \& Henry, 2004). In the current study, the PANAS was used to assess baseline mood.

\subsubsection{Toronto Alexithymia Scale}

(TAS-20; Bagby, Parker, et al., 1994; Bagby, Taylor, et al., 1994). The TAS20 consists of 20 items measuring three facets of alexithymia: difficulty identifying feelings (DIF; 7 items; e.g. "I am often confused about what emotion I am feeling"), difficulty describing feelings (DDF; 5 items, e.g. "It is difficult for me to find the right words for my feelings"), and externally-oriented thinking (EOT; 8 items, e.g. "Being in touch with emotions is essential"). Participants indicate their responses by selecting the answer that best describes them on a five-point scale ( $1=$ "strongly disagree" to 5 = "strongly agree"). DIF, DDF, and EOT subscale scores are combined to generate a total TAS-20 alexithymia score which can be used to determine the presence or absence of alexithymia. Scores $\geq 61$ indicate the presence of alexithymia, scores of 52 60 indicate 'possible' alexithymia and scores $\leq 51$ indicate the absence of alexithymia 
(Taylor, Bagby, \& Parker, 1992, 2006). The TAS-20 has good levels of validity, and in line with previous research (Bagby, Taylor, et al., 1994; Pinaquy et al., 2003), internal consistency was found to be high in the current study $(\alpha=.84)$. High levels of internal consistency were also found for the DIF $(\alpha=.83)$ and $\operatorname{DDF}(\alpha=.80)$, subscales. Consistent with previously reported levels (Larsen et al., 2006; Pike, 2013; Pinaquy et al., 2003), internal consistency of the EOT subscale was much lower ( $\alpha=$ $.55)$.

\subsubsection{UPPS-P}

(Cyders et al., 2007; Whiteside \& Lynam, 2001). The (negative) urgency, (lack of) premeditation, (lack of) perseverance, sensation seeking and positive urgency measure of impulsivity. The UPPS-P consists of 59 items designed to assess total levels of impulsivity as well as five facets: negative urgency (NU; 12 items; e.g. "When I feel bad, I will often do things I later regret in order to make myselffeel better now"); lack of premeditation (Prem; 11 items; e.g. "I have a reserved and cautious attitude toward life"); lack of perseverance (Pers; 10 items; e.g. "I generally like to see things through to the end"); sensation seeking (SS; 12 items; e.g. "I generally seek new and exciting experiences and sensations"), and positive urgency (PU; 14 items; e.g. "When I am in a great mood, I tend to get into situation that could cause me problems"). Participants respond on a four-point Likert type scale $(1=$ "agree strong" to $4=$ “disagree strongly"), with higher scores indicating greater impulsive tendencies. High levels of internal reliability were found for total UPPS-P $(\alpha=.94)$ and facet scores ranged from $\alpha=.86-.95)$. The UPPS-P has demonstrated good convergent and discriminant validity (Cyders \& Smith, 2007). 


\subsubsection{Beck Depression Inventory}

(BDI; Beck, Ward, Mendelson, Mock, \& Erbaugh, 1961; Beck, Steer, \& Brown, 1996; Beck, Steer, \& Carbin, 1988). The BDI is a 21-item measure assessing the severity of depressive symptoms in the past two weeks. Participants choose an answer that best describes them on a four-point Likert-type scale $(0=$ "not at all/no change" to $3=$ "increase/decrease in symptom"), with statements referring to "Sadness", "Pessimism" and "Past failure", as examples. Total scores range between 0-63, with scores between 0-13 indicating the presence of minimal depression, 14-19 mild depression, 20-28 moderate depression, and 29-63 severe depression (Wood, Williams, \& Lewis, 2010). The BDI has high levels of test-retest reliability and validity (Beck et al., 1961; Medley, Capron, Korte, \& Schmidt, 2013) and internal consistency was found to be high $(\alpha=.89)$ in the current study.

\subsubsection{Beck Anxiety Inventory}

(BAI; Beck, Epstein, Brown, \& Steer, 1988; Beck \& Steer, 1990). Consisting of 21-items, the BAI asks participants to rate how much they have been bothered by emotional, physiological, and cognitive symptoms of anxiety in the past week (e.g. "Nervous; Unable to relax; Unsteady"). Participants respond by selecting the answer that best describes them on a four-point Likert-type scale $(0=$ "not at all" to $3=$ "severely"). Total scores range between 0-63, with scores between 0-9 indicating the absence of anxiety, 10-18 mild to moderate anxiety, 19-29 moderate to severe anxiety, and scores between 30-63 severe anxiety (Beck \& Steer, 1990). In line with previous research (Beck, Epstein, et al., 1988), internal consistency of the measure was high in the current study $(\alpha=.91)$. 


\subsubsection{Emotional Eating Scale}

(EES; Arnow et al., 1995). The EES is a 25 item self-report measure to explore the urge to eat in response to a number of emotions (e.g. "Lonely" and "Angry"). Participants respond by selecting the answer that best describes them on a five-point scale ( $1=$ "no desire to eat" to $5=$ "an overwhelming urge to eat"). In addition to an EES total score, subscale scores can be calculated for anger/frustration (ang/frus; 11 items; e.g. "Resentful; Inadequate"), anxiety (anx; 9 items; e.g. "Shaky; Jittery"), and depression (dep; 5 items; e.g. "Blue; Worn out"). The EES demonstrated adequate to excellent levels of internal consistency (range $\alpha=.75-.93$ ) in the current study, and although initially created within a clinical population, it has been used extensively within general population samples (e.g. Moon \& Berenbaum, 2009; Price, Higgs, \& Lee, 2015). The EES was favoured over alternative measures of emotional eating (i.e. DEBQ) as it captures eating in response to a wide range of negative emotions.

\subsubsection{Three Factor Eating Questionnaire - Revised}

(TFEQ-R18; Karlsson, Persson, Sjöström, \& Sullivan, 2000): The TFEQ-R18 is a revised and shortened revision of the TFEQ (Stunkard \& Messick, 1985). Previous dichotomous responses were replaced with Likert type scales as the former were found to be restricting. Participants respond by selecting the answer that best describes them on a four-point Likert type scale ( 1 = "definitely true" to 4 = "definitely false"). Scores were calculated for each subscale as a proportion of the highest possible value and expressed on a scale of $0-100$, with higher scores indicating a greater tendency to restrain, lose control over eating and eat when in a negative mood. The TFEQ-R18 consists of three subscales: cognitive restraint (TFEQ-CR; 6 items; e.g. "I do not eat some foods because they make me fat"); uncontrolled eating (TFEQ-UE; 9 items; e.g. "I get so hungry that my stomach often seems like a bottomless pit"), and emotional 
eating (TFEQ-EE; 3 items; e.g. "When I feel anxious, I find myself eating"). 13 items are reversed scored and higher total scores are indicative of greater levels of cognitive restraint, uncontrolled eating and emotional eating. Although developed in an obese population, the TFEQ-R18 has been found to valid within general population samples and to have satisfactory levels of internal consistency (de Lauzon et al., 2004). In the current study, the following Cronbach's alphas were found: cognitive restraint $(\alpha=$ .86), uncontrolled eating ( $\alpha=.88)$, and emotional eating $(\alpha=.85)$. The TFEQ-R18 was selected to allow the inclusion of cognitive restraint and uncontrolled eating scores as control variables in the serial multivariate mediation analyses. Emotional eating scores were not utilised as the subscale is limited to only three items and does not sufficiently capture eating in response to a wide of emotions.

\subsubsection{Body Mass Index}

BMI was obtained by taking a measurement of height using a stadiometer in metres and three readings of weight to gain an average using WW digital scales in kilograms. BMI was calculated using the following equation in excel (weight $(\mathrm{kg}) /$ (height $\left.(\mathrm{m})^{2}\right)$ ).

\subsubsection{Delay Discounting Task}

A computer based monetary delay discounting task with nine delays (one day, two days, one week, two weeks, one month, three months, six months, nine months and one year) was used. Following a review of the literature, Price (2016) recommended that the longest time delay should not exceed one year. Price (2016) also recommended the use of a larger later reward (LLR) over $£ 10$ but under $£ 1000$ to avoid ceiling and floor effects, respectively. Consequently, £100 was deemed a suitable amount to detect differences over a one-year time frame. Participants had to choose whether they wanted the hypothetical larger, later amount (£100), or a smaller, 
sooner reward (SSR) which varied using a double limit procedure (random adjusting procedure) until the indifference point (IP) was calculated (description of the choice algorithm can be found in Richards, Zhang, Mitchell, \& de Wit, 1999). The IP is produced by using previous responses to narrow the range of values from which the value of the next set of comparisons are selected. The IP is a point at which the participant is indifferent between receiving a reward now or later, with a low IP indicating reduced ability to delay gratification and a tendency to make more impulsive choices. Following this, an outcome measure of behavioural discounting can be calculated using the IP points plotted on a graph (e.g. area under the curve).

To reduce participant error, the computer varied the SSR according to a double limit procedure, which prevented any single answer from controlling convergence to the IP (McHugh \& Wood, 2008). In addition, the location of presentation for the SSR and LLR varied so not all LLRs were presented on the same side for each delay, and vice versa with the SSRs. This method was used to limit response bias and to ensure participants were paying attention (e.g. due to the double limit procedure, participants could not simply click the same side of the screen/monetary amount without paying attention as this would extend the duration of the program until an IP was met). The presentation order of the delays for the LLR were random and all nine delays were presented in a single session. This task has been used in both clinical (McHugh \& Wood, 2008; Wood \& McHugh, 2013) and general population samples (Price, Higgs, Maw, \& Lee, 2016). 


\subsubsection{Procedure}

Ethical approval was obtained from the Department of Psychology Ethics Committee, College of Human and Health Sciences, Swansea University. Participants were recruited through the Department of Psychology's participant pool in exchange for course credit. The study was advertised as "An investigation into the relationship between alexithymia, impulsivity, emotional eating and delayed discounting". Participants were informed that the purpose of the study was to explore the relationship between our ability to describe and identify emotions, how we regulate specific behaviours such as eating, and how we make decisions on a daily basis. Participants were presented with a detailed information sheet before providing informed consent to partake. All participants began by completing the delay discounting task. Following this, participants were instructed to work through the selfreport measures which were presented via Survey Monkey (Palo Alto, California, USA). Upon completion, weight and height measurements were taken to allow BMI to be calculated. The study lasted for approximately 30 minutes and participants were thanked for their time upon completion.

\subsubsection{Statistical Analysis}

All statistical analyses were carried out using IBM SPSS Statistics 22.0 and PROCESS 2.16.3 (Hayes, 2013). Preliminary analysis examined the presence of outliers and the assumptions of normality were met. Pearson correlations were used to investigate the associations between measures (TAS-20, UPPS-P, EES, TFEQ, BMI, BDI and BAI). 


\subsubsection{Delay Discounting}

Delay discounting data was analysed for systematic response bias to check for systematic responding (Johnson \& Bickel, 2008). Algorithms created by Johnson \& Bickel (2008) were used to identify instances where IP's were not monotonically decreasing with delay. Specifically, there were two different criteria used to determine the presence of non-systematic data (i.e. outliers). The first criteria identified any IP (second delay and onwards) which was greater than the preceding IP by a magnitude larger than $25 \%$ of the LLR. In this study, this referred to any IP that was over $£ 25$ greater than the IP before (e.g. IP at 3 months was $£ 30.15$, and the IP at 6 months was $£ 60.15)$. Criteria 1 suggests that as the delay increases the reward value increases rather than decreases, and is often set at $20 \%$ to avoid being overly stringent and to allow for some variability in the data (Johnson \& Bickel, 2008). However, due to the LLR of the current study only being $£ 100$ and the maximum delay between IP's being three months, the magnitude was set to $25 \%$. The second criteria examined whether or not the last IP (one year) was less than the first IP (one day) by at least a magnitude equal to $5 \%$ of the LLR (£10; e.g. preferring to wait one year for $£ 100$ than receive $£ 95$ now, suggesting that delay had no effect on reward value). A magnitude of 5\% was adopted in the current study instead of the usual $10 \%$ due to the length of delay and total LLR. After analysing the data for non-systematic responses, delay discounting data for 22 participants were removed because of criteria 1, 23 for criteria 2, and two for violating both criteria. An additional four participants were removed as they were outliers on sat. $k$ and sat $Q$.

Area under the curve (AUC) was calculated for each participant by normalising the data point for each delay and subjective value using excel (for full equation see McHugh \& Wood, 2008). The delay was expressed as a proportion of the maximum 
delay (365 days). The subjective value was expressed as a proportion of the nominal amount (£100). The discount rate ranges from one ("no discounting") to zero ("total discounting;" McHugh \& Wood, 2008). However, AUC does not reflect the steepness of discounting for early relative to later delays, with research showing that the discount rate most often seen is greater over short than long term delays (Price, 2016; Wilkinson \& Klaes, 2012). Consequently, hyperbolic models have been implemented to describe the discount rates seen (Koffarnus, Jarmolowicz, Mueller, \& Bickel, 2013; Mazur, Stellar, \& Waraczynski, 1987). Of these, a one parameter $(k)$ hyperbolic model provides a superior fit (Mazur et al., 1987) and has the advantage of representing the steepness of a curve in a single value (Price, 2016). The formula used is as follows:

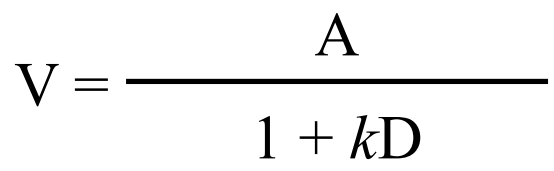

$\mathrm{V}$ is the indifference point, $\mathrm{A}$ is the larger later reward and $\mathrm{D}$ is the delay (in days), and $k$ is the free parameter for estimated steepness of the slope and time inconsistency. Typically, as the delay increases, the IP decreases as individuals are willing to accept less money immediately and favour waiting for a delayed reward. The decline in IP is time inconsistent, where it is steeper when delays are proximal (e.g. one day versus two days) and shallower when more distal (e.g. six months versus one year). Although, it should be noted that even though $k$ is considered as an appropriate fit to model data, Mazur and colleagues (1987) explored delay discounting in rats with delays of a few seconds which may not be suitable for explaining human behaviour (Price, 2016). The single parameter model $k$ was calculated using a least squares procedure on Gnuplot model fitting open source software (version 5.0; Williams \& Kelley, 2015). 
In contrast to $k$, two parameter models based on the competing neurobehavioural decision systems (CNDS; Koffarnus et al., 2013) model have been proposed (e.g. McKerchar, Green, \& Myerson, 2010; Peters, Miedl, \& Büchel, 2012; Yi, Landes, \& Bickel, 2009). The CNDS model argues that two neural systems are involved in choice behaviour: an executive decision system associated with lateral prefrontal cortex activation and an impulsive system related to limbic reward activity (Koffarnus et al., 2013). CNDS predicts that individual differences on one or both of these systems determine choice behaviour; with greater risk of delay discounting associated with a low-strength executive system and a high-strength impulsive reward system. A relatively novel two parameter model that represents the CNDS model is the saturating-hyperbolic model (Doya, 2008). Within this model, sat. $k$ represents the ability to imagine the future and relies on the executive decision system, and as with the single hyperbolic parameter function, it reflects the steepness of discounting from close to distant delays. The second parameter is sat. Q, representing the impulsive system and known as the reward utility function. A larger sat. Q value is indicative of a shallow utility curve and gestures that a reward is less appealing, whereas a smaller sat. Q suggests a steep reward curve and more appealing reward. Sat. Q is theorised as a related yet distinct process. When combined with sat. $k$, sat. Q reflects the utility of the reward after a delay, or in other words, the impulsive need and desire for a reward. For example, if the reward is desired as soon as possible, the sat. Q value will be large - suggesting any delay quickly devalues the reward. The formula for the calculation of sat. $k$ and sat. Q is:

$$
\mathrm{V}=\mathrm{A} *\left(\frac{\mathrm{A}}{\mathrm{A}+\mathrm{Q}}\right) *\left(\frac{\mathrm{A}}{1+k \mathrm{D}}\right)
$$


$\mathrm{V}$ is the indifference point, $\mathrm{A}$ is the larger later reward, $k$ is the hyperbolic temporal discounting parameter, Q is the reward utility parameter and D is the delay (in days). Sat. $k$ and sat. Q were calculated using Gnuplot model fitting open source software (version 5.0; Williams \& Kelley, 2015). AUC, $k$ and sat. $k$ were not normally distributed, and therefore, log transformations were performed. Log transformations successfully normalised the data for $k$ and sat. $k$ but not for AUC. Consequently, nonparametric correlation analysis (Spearman's) was conducted on AUC, whereas parametric correlation analysis (Pearson's) was performed on $\log k, \log$ sat. $k$ and sat. $Q$.

\subsubsection{Serial Multiple Mediation}

PROCESS is a SPSS macro for mediation, moderation, and conditional process modelling (Hayes, 2013). PROCESS was used as significant associations between variables are not a requirement. The current chapter introduces serial multiple mediation (PROCESS model 6) to analyse the direct and indirect pathways between alexithymia $(X)$ and emotional eating $(Y)$. Mediation aims to conceptualise the mechanism in which $X$, a predictor variable, influences $Y$, an outcome variable, centred around variations in $X$ leading to variation in mediator(s), $M$, which cause variations in $Y$ (Hayes, 2013). In terms of a serial multiple mediation model, the aim is to investigate the direct and indirect effects of $X$ on $Y$ while modelling a process in which $X$ causes $M_{1}$, which in turn causes $M_{2}$, and so forth, concluding with $Y$ (Hayes, 2013; see Figure 4). Serial mediation modelling allows for more than one simultaneous mediator to be entered at once. Mediators are assumed to have a direct effect on each other, and the predictor variable (independent; $X$ ) is assumed to influence mediators in a serial way that results in an influence on the outcome variable (dependent; $Y$; Cabello \& Fernández-Berrocal, 2015; Hayes, 2013). Serial mediation analysis allows 
examination of direct and indirect pathways between variables. As demonstrated in Figure 4, a total effect (c) refers to the relationship between the predictor variable and the outcome variable without controlling for the mediators; a direct effect ( $\left.c^{\prime}\right)$ refers to the relationship between the predictor and outcome variable after controlling for the mediators; a total indirect effect $(a b)$ is the role of the mediators in the relationship between the predictor and outcome variable, and finally, specific indirect effects (for example, $\left.a_{1} b_{1}, a_{2} b_{2}\right)$ refer to the role of a particular mediator or mediators in the relationship between the predictor and outcome variable. Using Figure 4 as an example, entering two mediators generates three specific indirect effects that can be obtained through the following pathways; (1) $M_{1}\left(a_{1} b_{1}\right)$, (2) $M_{1} M_{2}\left(a_{1} d_{21} b_{2}\right)$, and (3) $M_{2}\left(a_{2} b_{2}\right)$. A fourth pathway is the direct effect between $X$ and $Y$. Seven specific indirect effects are generated when entering three mediators in addition to the direct effect. Two and three mediator models were used in the current study. Contrast analysis can conduct pairwise comparisons between the significant specific indirect effects to determine the strongest indirect effect. Pairwise comparisons of indirect effects explored whether one indirect effect (e.g. $\left.a_{1} b_{1}\right)$ was significantly different from another (e.g. $\left.a_{1} d_{21} b_{2}\right)$.

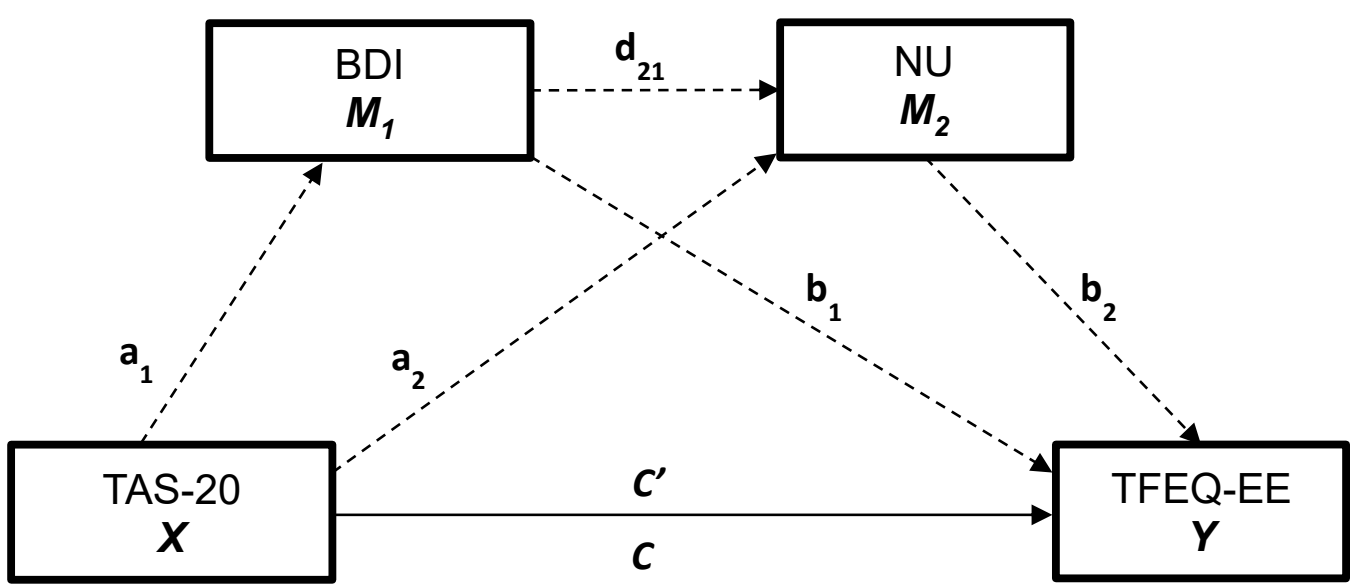

Figure 4. Serial mediation model of alexithymia (TAS-20) on emotional eating (TFEQ-EE) including depression (BDI) and negative urgency (NU) as mediators. 
Analyses used bootstrapping with 10,000 resamples and generated accelerated 95\% confidence intervals $(C I) .10,000$ bootstrapping resamples is sufficient and recommended for serial mediation models (Hayes, 2013) and reduces type 1 error (false positive; finding a significant result when one is not present). To test for significance for total indirect effects and specific indirect effects, the 95\% CI of the point estimate must not cross zero. The same significant classification is adopted when interpreting the contrast pairwise comparisons.

32 models were analysed for direct and specific indirect effects (see Table 1). To recap, a direct effect $\left(c^{\prime}\right)$ is the relationship between $X$ and $Y$ controlling for all mediators and a specific indirect effect (e.g. $\left.a_{1} b_{1}, a_{1} d_{21} b_{2}\right)$ is the relationship between $X$ and $Y$ via a particular mediator or mediators. Using Model 1 (see Table 1) as an example and Figure 4, the direct and indirect effects analysed can be demonstrated. Total alexithymia scores were entered as the predictor variable $(X)$ and emotional eating scores as the outcome variable $(Y)$. Multiple mediators were then entered in the following order; affect and impulsivity (e.g. Model 1; TAS-20 $\rightarrow$ BDI $\rightarrow$ NU $\rightarrow$ TFEQ-EE). The following indirect effects were therefore examined: via BDI alone $\left(a_{1} b_{1}\right)$, via BDI and NU $\left(a_{1} d_{21} b_{2}\right)$ and via NU only $\left(a_{2} b_{2}\right)$. The remaining models followed this structure substituting the TAS-20 subscales for $X$, BAI for affect at $M_{l}$ and two different measures of emotional eating at $Y$. Models 17-32 expand the models to include BMI at $Y$ and emotional eating as a mediator at $M_{3}$ (See Figure 5; Table 1). All models controlled for age, sex, cognitive restraint (TFEQ) and uncontrolled eating (TFEQ), and models 1-16 also controlled for BMI. 


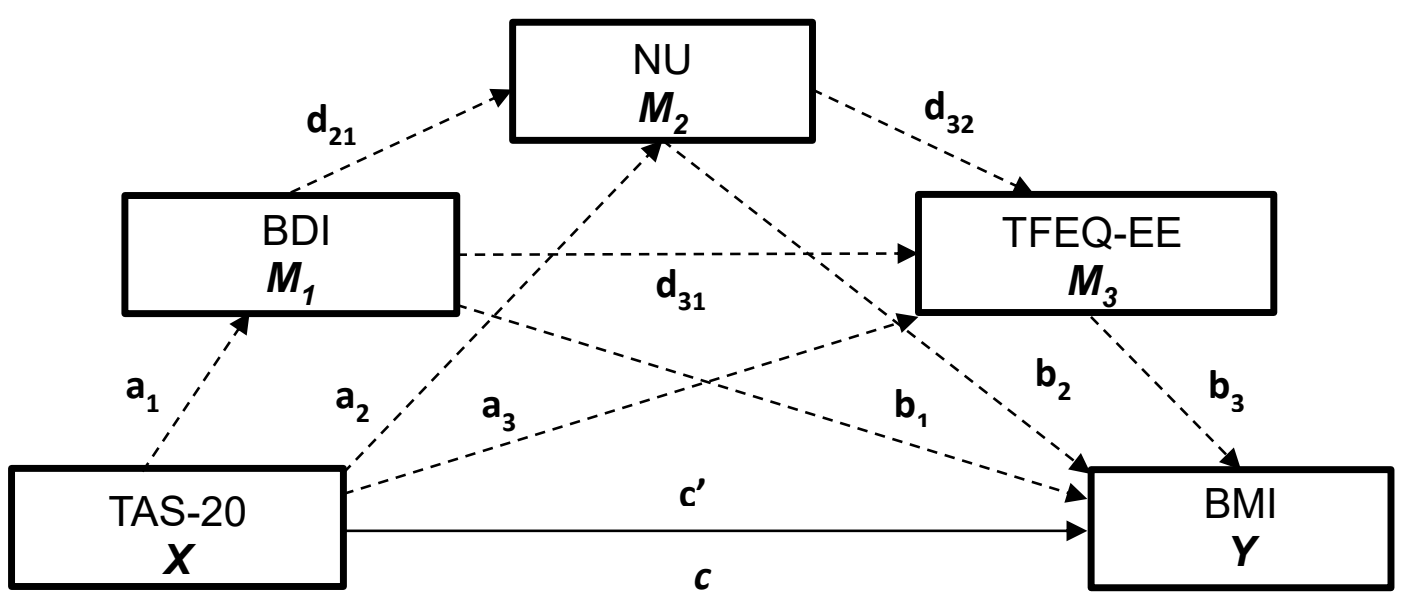

Figure 5. Serial mediation of alexithymia (TAS-20) on BMI including depression (BDI), negative urgency (NU) and emotional eating (TFEQ-EE) as mediators.

Self-reported negative urgency was entered as the measure of impulsivity in the current analysis. The delay discounting variables were not used for the following reasons: a) the final sample with suitable delay discounting data was 75 participants, resulting in possible power issues; b) the variables were not normally distributed, and c) the theoretical model was driven specifically by individuals acting rashly in response to negative affect (negative urgency) rather than impulsivity more broadly. 
Table 1. The serial multivariate mediation pathways analysed in Study One.

\begin{tabular}{|c|c|c|c|}
\hline Model & Pathway tested & & \\
\hline 1 & $\mathrm{TAS}-20 \rightarrow \mathrm{BDI} \rightarrow \mathrm{NU} \rightarrow \mathrm{TFEQ}-\mathrm{EE}$ & 17 & $\rightarrow \mathrm{BMI}$ \\
\hline 2 & $\mathrm{DIF} \rightarrow \mathrm{BDI} \rightarrow \mathrm{NU} \rightarrow \mathrm{TFEQ}-\mathrm{EE}$ & 18 & $\rightarrow \mathrm{BMI}$ \\
\hline 3 & $\mathrm{DDF} \rightarrow \mathrm{BDI} \rightarrow \mathrm{NU} \rightarrow \mathrm{TFEQ}-\mathrm{EE}$ & 19 & $\rightarrow \mathrm{BMI}$ \\
\hline 4 & $\mathrm{EOT} \rightarrow \mathrm{BDI} \rightarrow \mathrm{NU} \rightarrow \mathrm{TFEQ}-\mathrm{EE}$ & 20 & $\rightarrow \mathrm{BMI}$ \\
\hline 5 & $\mathrm{TAS}-20 \rightarrow \mathrm{BDI} \rightarrow \mathrm{NU} \rightarrow \mathrm{EES}$ & 21 & $\rightarrow \mathrm{BMI}$ \\
\hline 6 & $\mathrm{DIF} \rightarrow \mathrm{BDI} \rightarrow \mathrm{NU} \rightarrow \mathrm{EES}$ & 22 & $\rightarrow \mathrm{BMI}$ \\
\hline 7 & $\mathrm{DDF} \rightarrow \mathrm{BDI} \rightarrow \mathrm{NU} \rightarrow \mathrm{EES}$ & 23 & $\rightarrow \mathrm{BMI}$ \\
\hline 8 & $\mathrm{EOT} \rightarrow \mathrm{BDI} \rightarrow \mathrm{NU} \rightarrow \mathrm{EES}$ & 24 & $\rightarrow \mathrm{BMI}$ \\
\hline 9 & $\mathrm{TAS}-20 \rightarrow \mathrm{BAI} \rightarrow \mathrm{NU} \rightarrow \mathrm{TFEQ}-\mathrm{EE}$ & 25 & $\rightarrow \mathrm{BMI}$ \\
\hline 10 & $\mathrm{DIF} \rightarrow \mathrm{BAI} \rightarrow \mathrm{NU} \rightarrow \mathrm{TFEQ}-\mathrm{EE}$ & 26 & $\rightarrow \mathrm{BMI}$ \\
\hline 11 & $\mathrm{DDF} \rightarrow \mathrm{BAI} \rightarrow \mathrm{NU} \rightarrow \mathrm{TFEQ}-\mathrm{EE}$ & 27 & $\rightarrow \mathrm{BMI}$ \\
\hline 12 & $\mathrm{EOT} \rightarrow \mathrm{BAI} \rightarrow \mathrm{NU} \rightarrow \mathrm{TFEQ}-\mathrm{EE}$ & 28 & $\rightarrow \mathrm{BMI}$ \\
\hline 13 & $\mathrm{TAS}-20 \rightarrow \mathrm{BAI} \rightarrow \mathrm{NU} \rightarrow \mathrm{EES}$ & 29 & $\rightarrow \mathrm{BMI}$ \\
\hline 14 & $\mathrm{DIF} \rightarrow \mathrm{BAI} \rightarrow \mathrm{NU} \rightarrow \mathrm{EES}$ & 30 & $\rightarrow \mathrm{BMI}$ \\
\hline 15 & $\mathrm{DDF} \rightarrow \mathrm{BAI} \rightarrow \mathrm{NU} \rightarrow \mathrm{EES}$ & 31 & $\rightarrow \mathrm{BMI}$ \\
\hline 16 & $\mathrm{EOT} \rightarrow \mathrm{BAI} \rightarrow \mathrm{NU} \rightarrow \mathrm{EES}$ & 32 & $\rightarrow \mathrm{BMI}$ \\
\hline
\end{tabular}

BAI $=$ Beck anxiety inventory, $\mathrm{BDI}=$ Beck depression inventory, $\mathrm{BMI}=$ body mass index, DIF = difficulty identifying feelings, DDF = difficulty describing feelings, EES $=$ total $\mathrm{EES}, \mathrm{EOT}=$ externally oriented thinking, $\mathrm{NU}=$ negative urgency, $\mathrm{TAS}-20=$ total Toronto alexithymia scale, TFEQ-EE = emotional eating TFEQ subscale.

\subsection{Study One: Results}

\subsubsection{Descriptive Statistics}

Descriptive statistics for Study One for the full sample $(N=125)$ can be found in Table 2. The PANAS revealed a normal distribution of positive and negative affect and no outliers. 


\subsubsection{Delay Discounting}

A final sample of $75(\mathrm{~F}=68)$ systematic responders were used in all analysis including delay discounting data. The mean age of this sub-sample was 21.23 years $(S D=3.56$, range $=18.27-36.87$ years $)$ and BMI was $24.37(S D=4.27$, range $=17.4-$ 36.76)). The descriptive statistics for the delay discounting variables are presented in Table 3. Non-systematic responders reported significantly less income ( $M=873.86$, $S D=1976.68)$ compared to systematic responders $(M=2686.67, S D=4591.25$; $t(108.23)=3.03, p=.003)$. The age difference between the non-systematic responders $(M=20.26, S D=1.99)$ and the systematic responders $(M=21.23, S D=3.56)$ approached significance $(t(119.79)=1.95, p=.053$. There were no significant differences between participants who responded non-systematically and those who responding systematically for years of education $(t(120.53)=1.56, p=.122)$, BMI $(t(123)=1.18, p=.162)$, total UPPS-P $(t(123)=.9 p=.371)$, PA $(t(123)=-.12, p=$ .908 , or NA $(t(123)=-1.37, p=.173)$. There was a significant gender difference with more females in the systematic responders group, $X^{2}=3.91(1, \mathrm{~N}=125), p=.048, V$ $=.177$. 
Table 2. Mean, standard deviation and range for each study variable in Study One.

\begin{tabular}{|c|c|c|c|}
\hline Measure & Mean & $S D$ & Range \\
\hline $\mathrm{PA}$ & 30.94 & 7.52 & $10-44$ \\
\hline NA & 19.76 & 6.95 & $10-37$ \\
\hline DIF & 14.26 & 5.54 & $7-30$ \\
\hline DDF & 12.61 & 4.74 & $5-25$ \\
\hline EOT & 18.64 & 4.08 & $10-30$ \\
\hline TAS-20 & 45.51 & 11.33 & $25-76$ \\
\hline NU & 28.26 & 7.01 & $12-46$ \\
\hline PU & 26.36 & 9.06 & $14-56$ \\
\hline Prem & 22.00 & 5.42 & $11-36$ \\
\hline Pers & 19.91 & 4.87 & $10-34$ \\
\hline SS & 34.46 & 8.19 & $12-48$ \\
\hline UPPS-P & 130.99 & 24.10 & 69-202 \\
\hline BDI & 10.14 & 8.08 & $0-36$ \\
\hline BAI & 10.10 & 9.49 & $0-44$ \\
\hline Ang/Frus & 18.91 & 8.25 & $9-36$ \\
\hline Anx & 16.00 & 5.68 & $9-36$ \\
\hline Dep & 13.69 & 4.36 & $5-24$ \\
\hline EES & 48.60 & 16.41 & $26-108$ \\
\hline TFEQ-CR & 44.15 & 24.19 & $0-100$ \\
\hline TFEQ-UE & 45.11 & 22.90 & $00-100$ \\
\hline TFEQ-EE & 42.58 & 29.15 & $0-100$ \\
\hline
\end{tabular}

Ang/Frus = anger and frustration EES subscale, Anx = anxiety EES subscale, $\mathrm{BAI}=$ Beck anxiety inventory, BDI $=$ Beck depression inventory, DDF $=$ difficulty describing feelings, Dep = depression EES subscale, DIF = difficulty identifying feelings, EOT = externally oriented thinking, EES = total EES, NA = negative affect, $\mathrm{NU}=$ negative urgency, $\mathrm{PA}=$ positive affect, Pers = (lack of $)$ perseverance, Prem = (lack of) premeditation, $\mathrm{PU}=$ positive urgency, $\mathrm{SS}=$ sensation seeking, TAS-20 = total Toronto alexithymia scale, TFEQ-CR = cognitive restraint TFEQ subscale, TFEQ-EE = emotional eating TFEQ subscale, TFEQ-UE = uncontrolled eating TFEQ subscale, UPPS-P = UPPS-P total score. 
Table 3. Mean, standard deviation and range for each delay discounting parameter.

\begin{tabular}{lccc}
\hline Variable & Mean & $S D$ & Range \\
\hline AUC & 61.21 & 22.38 & $11.23-95.58$ \\
$k$ & .009 & .014 & $.0003-.0791$ \\
Sat. $k$ & .0082 & .0132 & $.0001-.0652$ \\
Sat. $Q$ & 4.537 & 5.727 & $-10.88-16.11$ \\
\hline
\end{tabular}

$\mathrm{AUC}=$ area under the curve.

\subsubsection{Correlational Analysis}

\subsubsection{Correlations between alexithymia, negative affect, negative urgency,} emotional eating and BMI

Pearson's correlations were conducted to initially explore the relationships between alexithymia, impulsivity, affect, emotional eating and BMI. Significant positive correlations were found between EES total scores and TAS-20 total scores $(r$ $=.176, p=.049)$ and DIF subscale scores $(r=.203, p=.024$; see Table 4$)$. The negative urgency facet was also significantly positively correlated with EES total scores $(r=$ $.385, p \leq .001)$, and similar results were found for the emotional eating subscale of the TFEQ $(r=.380, p \leq .001)$. Significant positive correlations were also found between BDI, BAI and emotional eating scores as measured by the EES and TFEQ-EE (see Table 4). There were no significant correlations between BMI and any of the other variables. After applying a Bonferroni correction $(p<.0011)$, only the correlations between emotional eating, negative urgency and affect remained significant (see Table 4). Pearson's correlations for individual subscales of the TAS-20, EES and UPPS-P can be found in Appendix $\mathbf{J}$. 
Table 4. Pearson correlations between alexithymia, negative urgency, negative affect, emotional eating and B 1. 2. 3. 4. 5. 6. 1. DIF

2. DDF $.638 * * *$

3. EOT $.270^{* *}$ $.331 * * *$

4. TAS-20 $.853^{* * *}$ $.849 * * *$ $.631 * * *$

5. NU $.422 * * *$ $.234 * *$ .102 $.341 * * *$

6. BDI $.529 * * *$ $.397 * * *$ .037 $.438^{* * * *}$ $.379 * * *$

7. BAI $.437 * * * \quad .308 * * *$ .116

$.384 * * *$ $.304 * * *$ $.639 * * *$

8. EES $.203 *$ .085 .115

$.176^{*}$ $.385^{* * *}$ $.265^{* *}$

9. TFEQ-EE .074 .018 .090 .076 $.328 * * *$ .069

10. BMI .151 .078 $-.067$ .083 .077 .065

$\mathrm{BAI}=$ Beck anxiety inventory, $\mathrm{BDI}=$ Beck depression inventory, $\mathrm{BMI}=$ body mass index, DDF = difficulty identifying feelings, EES = total EES, EOT = externally oriented thinking, NU = negative urgency, TAS -20 $\mathrm{EE}=$ emotional eating TFEQ subscale. $*=p<.05, * *=p<.01, * * *=p<.001$. 


\subsubsection{Correlations between delay discounting, alexithymia, negative urgency, emotional eating and BMI.}

Pearson's correlational analysis were conducted on the sub-sample of systematic responders $(n=75)$ for $\log k, \log$ Sat. $k$ and Sat. $Q$ with Spearman's correlational analysis performed for AUC. There were no significant correlations between any of the personality factors and delay discounting variables (see Table 5). A strong significant positive correlation was found between $\log k$ and $\log$ Sat. $k(r=$ $.986, p<.001)$.

Table 5. Correlations between delay discounting parameters and BMI, emotional eating, alexithymia and negative urgency.

\begin{tabular}{lcccc}
\hline & AUC & $k$ & Sat. $k$ & Sat. $Q$ \\
\hline BMI & .077 & .015 & -.064 & .113 \\
EES & -.039 & -.053 & -.043 & -.016 \\
TFEQ-EE & .104 & -.126 & -.127 & .005 \\
DIF & .031 & -.015 & -.033 & .005 \\
DDF & -.032 & .002 & -.009 & -.048 \\
EOT & -.062 & .039 & .041 & .016 \\
TAS-20 & -.025 & .007 & -.005 & -.013 \\
NU & -.138 & .147 & .126 & .025 \\
\hline AUC & a & &
\end{tabular}

$\overline{\mathrm{AUC}}=$ area under the curve, $\mathrm{BMI}=$ body mass index, $\mathrm{DDF}=$ difficulty describing feelings, DIF = difficulty identifying feelings, EES = total EES, EOT = externally oriented thinking, NU = negative urgency, TAS-20 = total Toronto alexithymia scale, TFEQ-EE = emotional eating TFEQ subscale.

\subsubsection{Serial Multiple Mediation for Emotional Eating}

Model 1 entered total alexithymia scores as the predictor variable $(X)$, with BDI scores as $M_{1}$, NU as $M_{2}$, and TFEQ scores as $Y$. No significant direct effect was found, but two significant specific indirect effects emerged via NU $\left(a_{2} b_{2}\right), B=.1028$, 
$C I=.0065-.2982$, and via BDI and NU $\left(a_{1} d_{21} b_{2}\right), B=.0425, C I=.0025-.1598$. Contrast analysis revealed no significant differences in the strength of the effects, $B=-.0603$, $C I=-.2719-.0589$

Models 2-4 entered DIF, DDF and EOT as X. No significant direct effects emerged across Models 2-4 and no significant specific indirect effects were found in Model 4 (EOT as $Y$ ). However, significant specific indirect effects were found in Model $2(\mathrm{DIF}$ as $X)$ via NU $\left(a_{2} b_{2}\right), B=.3214, C I=.0500-.8197$, and via BDI and NU in Model $3(\mathrm{DDF}$ as $X)\left(a_{1} d_{21} b_{2}\right), B=.1081, C I=.0082-.3700$.

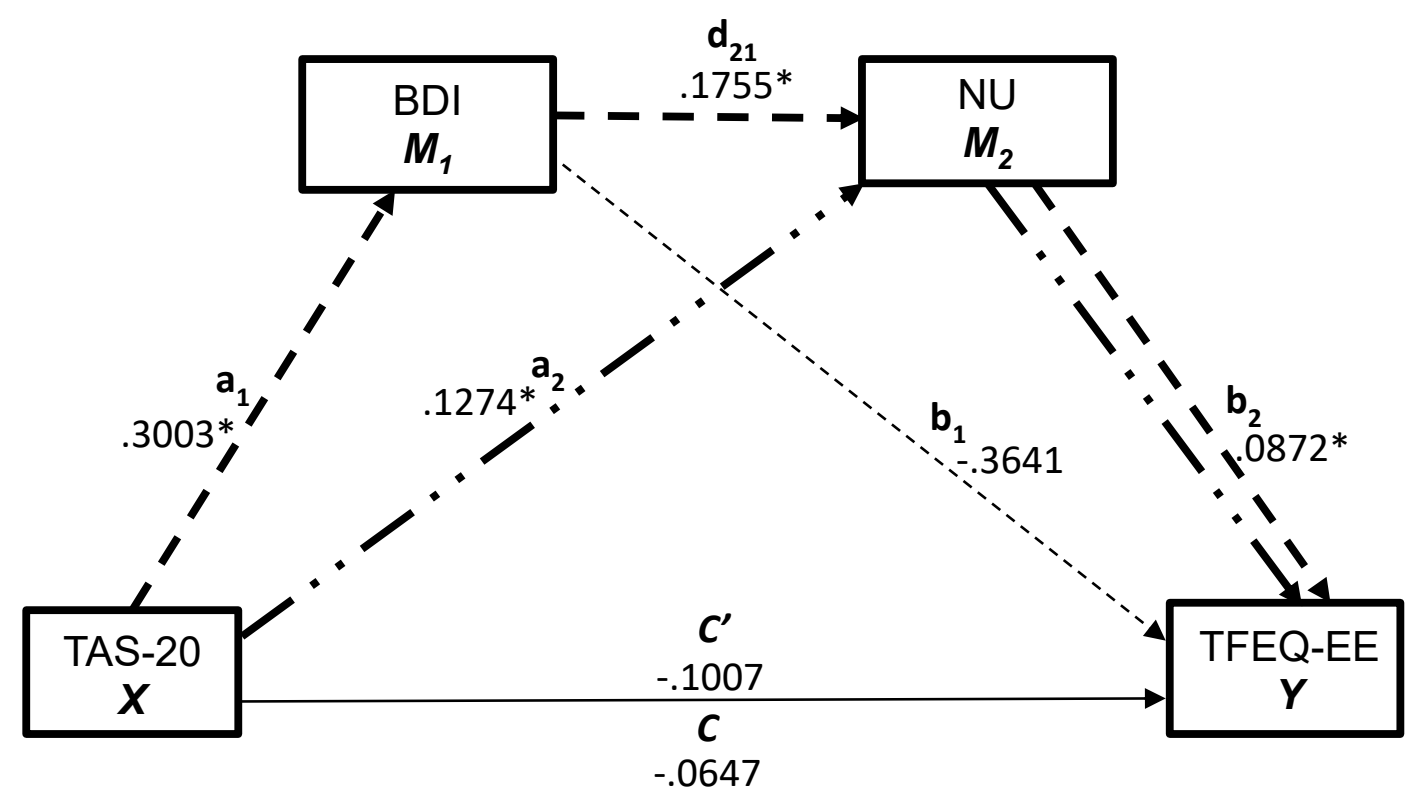

Figure 6. Example PROCESS serial multiple mediation output to identify the direct and indirect effects between alexithymia (TAS-20) and emotional eating (TFEQ-EE) including depression (BDI) and negative urgency (NU) as mediators.

Model 5 replaced emotional eating as measured by the TFEQ with total scores from the EES at $Y$ and entered total alexithymia scores at $X$. There was no significant direct effect between alexithymia total scores and EES, but there were two significant specific indirect effects; the first via NU $\left(a_{2} b_{2}\right), B=.0568, C I=.0015-.1725$ and the 
second via BDI and NU $\left(a_{1} d_{21} b_{2}\right), B=.0235, C I=.0019-.0841$. Contrast analysis revealed no significant difference in the strength of the two specific indirect effects, $B$ $=-.0333, C I=-.1583-.0288$.

Models 6-8 entered DIF, DDF and EOT as $X$ respectively. Similar to Models $2-4$, there were no significant direct effects between each of the alexithymia subscales and emotional eating. Model 6 (DIF as $X$ ) found a significant total indirect effect, $(a b)$, $B=.3777, C I=.0132-.7538$ and a significant specific indirect effect via NU $\left(a_{2} b_{2}\right), B$ $=.1680, C I=.0187-.4449$. DDF as $X$ (Model 7) revealed a significant indirect effect via BDI and NU $\left(a_{1} d_{21} b_{2}\right), B=.0619, C I=.0075-.1964$, whereas no specific indirect effects were found when EOT was entered as $X$ (Model 8).

Model 9 entered total alexithymia scores as $X$ and emotional eating as measured by the TFEQ as $Y$. It also replaced BDI scores at $M_{l}$ with BAI scores. There was no significant direct effect between total alexithymia scores and emotional eating, but there were two significant specific indirect pathways. The first was via NU $\left(a_{2} b_{2}\right)$, $B=.1269, C I=.0203-.3191$, and the second via BAI and NU $\left(a_{1} d_{21} b_{2}\right), B=.0295, C I$ $=.0003-.1218$. Contrast analysis revealed no significant difference in the strength of the two indirect effects, $B=-.0974, C I=-.3042-.0009$.

Models 10-12 entered the subscales of the TAS-20 as $X$ and no significant direct effects were found. Model 10 (DIF as $X$ ) revealed one significant specific indirect effect via NU $\left(a_{2} b_{2}\right), B=.3766, C I=.0927-.8554$. Three significant specific indirect effects were found in Model 11 (DDF as $X$ ). The first was via BAI $\left(a_{1} b_{1}\right), B=$ $-.3709, C I=-.9049--.0136$, the second was via NU $\left(a_{2} b_{2}\right), B=.1919, C I=.0050-.5998$, and the third was via BAI and NU $\left(a_{1} d_{21} b_{2}\right), B=.0707, C I=.0065-.2756$. Contrast analysis revealed that the indirect effect via BAI was significantly stronger than via $\mathrm{NU}(B=-.5629, C I=-1.1737--.1013)$ and via BAI and NU $(B=-.4416, C I=-1.0694-$ 
-.0491). There was no significant difference in the strength of the indirect effects via $\mathrm{NU}$ and via BAI and NU ( $B=-.1212, C I=-.5240-.1049)$. No significant specific indirect effects were found in Model 12 (EOT as $X$ ).

Model 13 entered total alexithymia scores as $X$ and EES scores at $Y$. There was no significant direct effect, but two significant specific indirect effects were found: the first via NU $\left(a_{2} b_{2}\right), B=.0646, C I=.0060-.1787$, and the second via BAI and NU $\left(a_{1} d_{21} b_{2}\right), B=.0150, C I=.0003-.0632$. Contrast analysis revealed no significant difference in the strength of the indirect effects, $B=-.0496, C I=-.1726-.0048$.

Models 14-16 entered the subscales of the TAS-20 as $X$ and no significant direct effect was found between DIF, DDF or EOT and emotional eating. There was a significant total indirect effect, $B=.3635, C I=.0811-.7573$ and specific indirect effect via NU $\left(a_{2} b_{2}\right), B=.1833, C I=.0242-.452$ in Model 14 (DIF as $\left.X\right)$. A similar pattern was seen for Model 15 (DDF as $X$ ), with a significant total indirect effect, $B=.2357, C I=.0463$ .6030 and a significant indirect effect via BAI and NU $\left(a_{1} d_{21} b_{2}\right), B=.0367, C I=.0025-$ .1394. No significant specific indirect effects were found in Model 16 (EOT as $X$ ). 


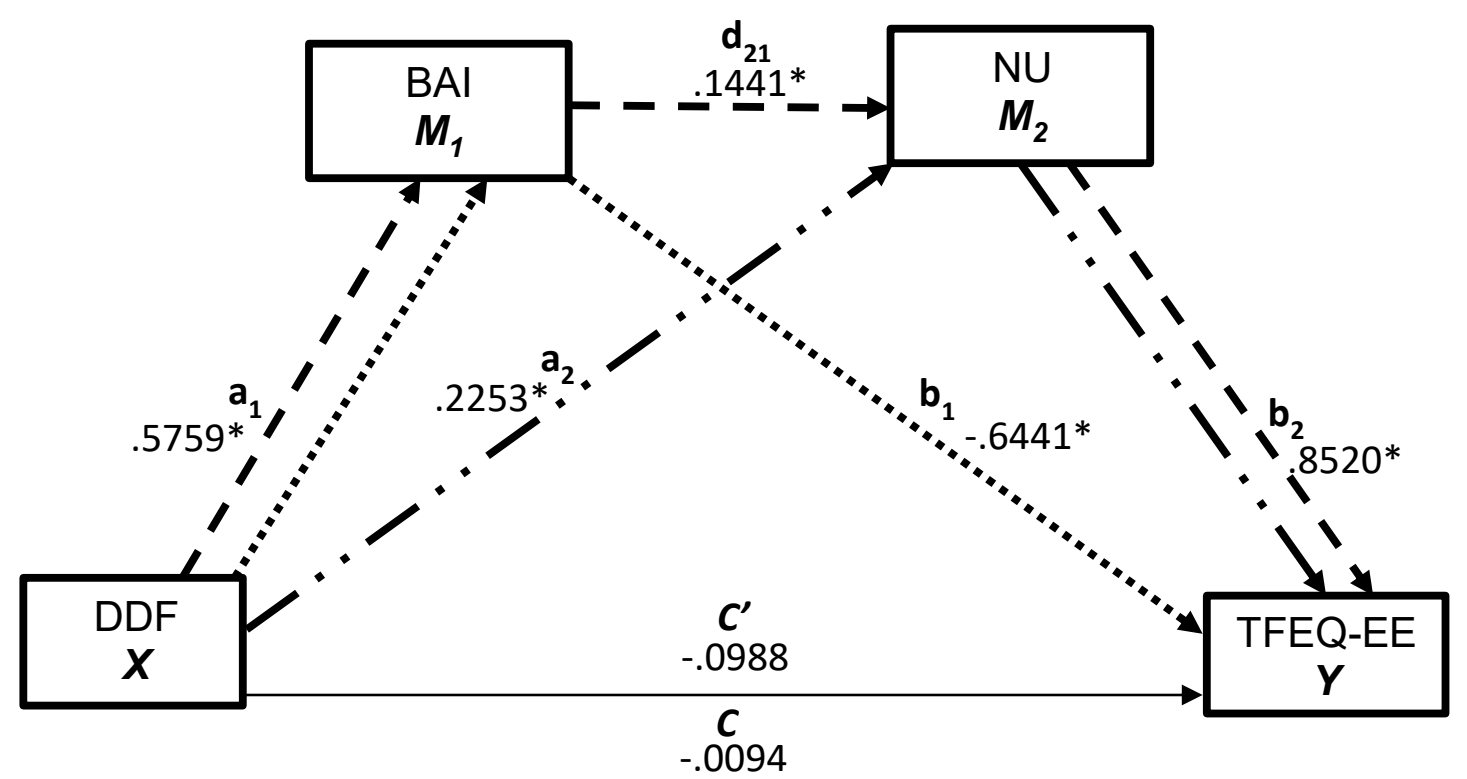

Figure 7. Example PROCESS serial multiple mediation output to identify the direct and indirect effects between difficulty describing feelings (DDF) and emotional eating (TFEQ-EE) including anxiety (BAI) and negative urgency (NU) as mediators.

\subsubsection{Exploratory analysis of serial multiple mediation for BMI}

Models 17-32 incorporated BMI as the outcome variable ( $Y$; see Table 1). To recap section 2.3.4.2., BMI was entered as $Y$, with total TAS-20 and subscales scores (DIF, DDF, EOT) entered as $X$. Three mediators were added in serial: negative affect $\left(M_{1}\right.$; separate models for depression and anxiety); negative urgency $\left(M_{2}\right)$, and emotional eating (EE; $M_{3}$; separate models for the EES and TFEQ). This created seven possible indirect effects: via BDI alone $\left(a_{1} b_{1}\right)$; via BDI and NU $\left(a_{1} d_{21} b_{2}\right)$; via NU only $\left(a_{2} b_{2}\right)$; via NU and EE $\left(a_{2} d_{32} b_{3}\right)$; via BDI and EE $\left(a_{1} d_{31} b_{3}\right)$; via EE only $\left(a_{3} b_{3}\right)$, and via BDI, NU and EE $\left(a_{1} d_{21} d_{32} b_{3}\right)$.

Model 17 included total alexithymia scores as $X$, BDI scores as $M_{1}$ and emotional eating as measured by the TFEQ as $M_{3}$. No significant direct effect was found, but a specific indirect effect via BDI, NU and TFEQ-EE approached 
significance $\left(a_{1} d_{21} d_{32} b_{3}\right), B=.0009, C I=.0000-.0059$. Hayes (2013) states that for an effect to be significant, the confidence intervals must not contain 0 .

Models 18-20 explored the subscales of the TAS-20 as $X$. Model 18 entered DIF at $X$ and found a significant direct effect between DIF and BMI $\left(c^{\prime}\right), B=.1694$, $C I=.0194-.3194$. In addition, one significant specific indirect effect was found via NU and TFEQ-EE $\left(a_{2} d_{32} b_{3}\right), B=.0074, C I=.0001-.0315$ and a second via BDI, NU, and TFEQ-EE $\left(a_{1} d_{21} d_{32} b_{3}\right), B=.0021, C I=.0000-.0129$, approached significance. Models 19 and 20 (DDF and EOT as $X$ respectively) found no significant direct or indirect effects.

Model 21 included emotional eating as measured by the EES as $M_{3}$ and entered total alexithymia scores as $X$. Similar to Model 17 , there was no significant direct effect but a specific indirect effect via BDI, NU and EES $\left(a_{1} d_{21} d_{32} b_{3}\right)$ approached significance, $B=.0008, C I=.0000-.0050$.

Models 22-24 tested DIF, DDF and EOT as X. Similar to Model 18, there was a significant direct effect between DIF and BMI ( $\left.c^{\prime}\right), B=.1608, C I=.0107-.3109$, as well specific indirect effect via BDI, NU and EES $\left(a_{1} d_{21} d_{32} b_{3}\right)$ that approached significance, $B=.0016, C I=.0000-.0103$, in Model 22 (DIF as $X)$. In line with Models 19 and 20, no significant direct or indirect effects were found in Models 23 and 24 when DDF and EOT subscales were entered as $X$ respectively.

Model 25 was the first model in the current set of models using BAI at $M_{1}$. It also used total alexithymia scores as $X$ and emotional eating as measured by the TFEQ as $M_{3}$. There was no significant direct effect, but there was a significant specific indirect effect via NU and TFEQ-EE $\left(a_{2} d_{21} b_{3}\right), B=.0034, C I=.0002-.0131$. A second indirect effect via BAI, NU and TFEQ-EE $\left(a_{1} d_{21} d_{32} b_{3}\right), B=.0008, C I=.0000-.0051$, approached significance. 
Models 26-28 entered DIF, DDF and EOT as $X$ and no significant direct effects on BMI were found. A significant specific indirect effect via NU and TFEQ-EE $\left(a_{2} d_{32} b_{3}\right)$ was found in Model 26 (DIF as $\left.X\right), B=.0102, C I=.0009-.0353$. Three significant indirect effects were found in Model 27 (DDF as $X$ ): via BAI, $\left(a_{1} b_{1}\right), B=$ $.0427, C I=.0014-.1162$; via BAI, NU and TFEQ-EE $\left(a_{1} d_{21} d_{32} b_{3}\right), B=.0019, C I=$ $.0001-.0119)$, and via NU and TFEQ-EE $\left(a_{2} d_{32} b_{3}\right), B=.0052, C I=.0001-.0243$. Contrast analysis revealed that the effect via BAI was significantly stronger than the effects via BAI, NU and TFEQ-EE, $B=.0408, C I=.0003-.1118$, but not via NU and TFEQ-EE, $B=.0376, C I=-.0035-.1106$. There was no significant difference between the indirect effects via BAI, NU and TFEQ-EE and via NU and TFEQ-EE, $B=-.0033$, $C I=-.0224-.0015$. EOT as $X$ (Model 28) had no significant indirect effect on BMI.

Models 29-32 found no significant direct or indirect effects when emotional eating as measured by the EES was entered at $M_{3}$. 


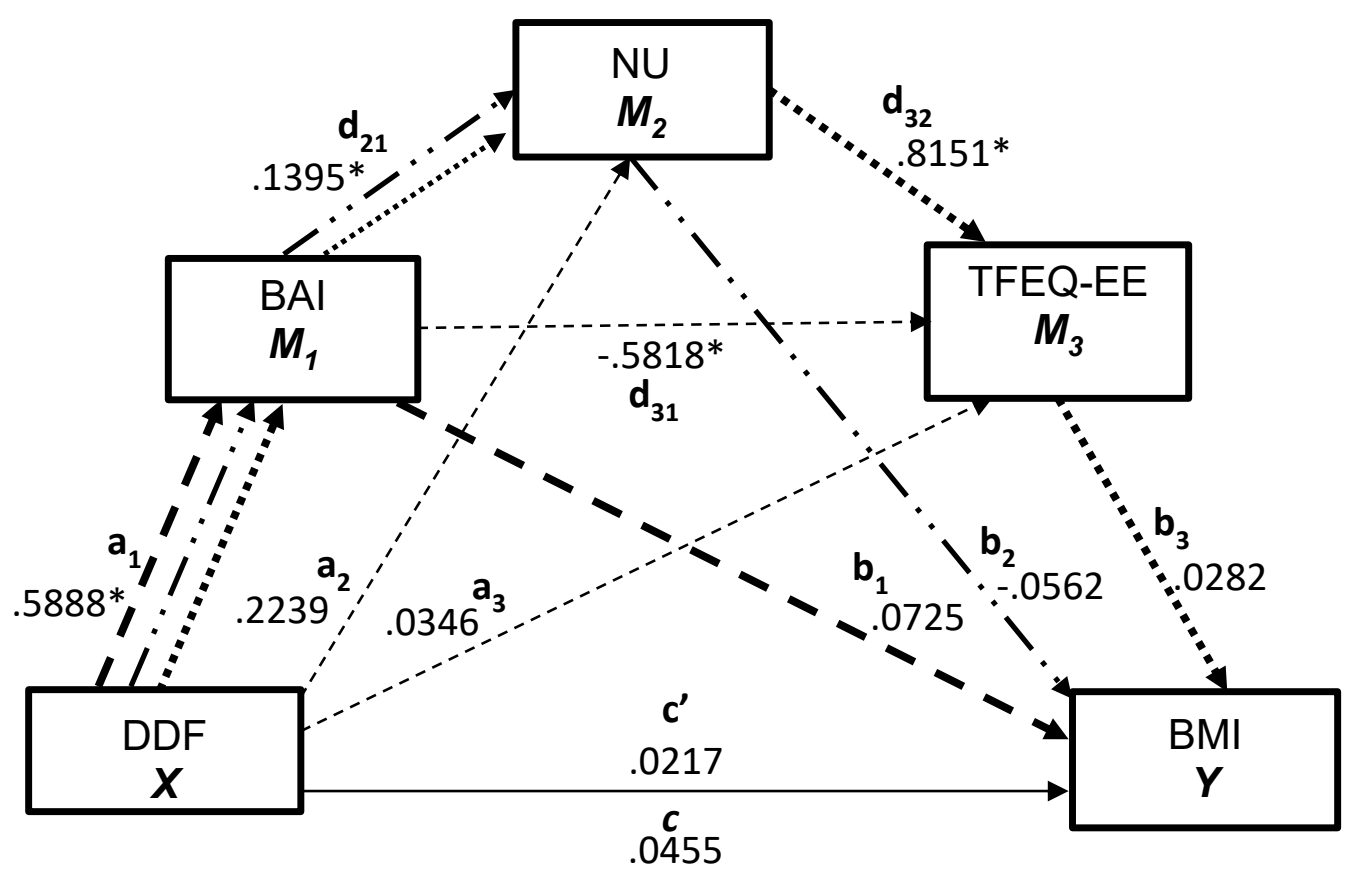

Figure 8. Example PROCESS serial multiple mediation output to identify the direct and indirect effects between difficulty describing feelings (DDF) and body mass index (BMI) including anxiety (BAI), negative urgency (NU) and emotional eating as mediators.

\subsection{Study One: Discussion}

The overarching aim of Study One was to comprehensively explore the role of emotional dysregulation in emotional eating, and in turn, BMI. Consistent with previous research (e.g. Larsen et al., 2006; Nguyen-Rodriguez et al., 2009; Pike, 2013; Spence \& Courbasson, 2012), a number of significant correlations were found between alexithymia, emotional eating, impulsivity, negative affect and BMI. However, no significant correlations were found between BMI and emotional eating as measured by the EES and TFEQ. Although it seems logical that emotional eating and BMI should be significantly correlated, previous research has also reported similar findings (e.g. Adriaanse et al., 2011; Snoek, van Strien, et al., 2007). In addition, alexithymia 
was only significantly correlated with emotional eating when measured by the EES, with higher levels of alexithymia and greater difficulty identifying emotions associated with higher levels of emotional eating and eating in response to feelings of anger and frustration (although these were not significant when applying a Bonferroni adjusted alpha level). Overall, levels of impulsivity were significantly and positively related to emotional eating, and specifically, eating in response to depression. Negative urgency was significantly correlated with emotional eating scores (EES and TFEQ), suggesting that individuals who act rashly in response to negative affect (negative urgency) may turn to food as a way to alleviate their distress. Higher levels of depression and anxiety were also significantly correlated with higher levels of overall emotional eating, as well as eating in response to feelings of anger/frustration and anxiety. A number of significant correlations were found between alexithymia and impulsivity as measured by the UPPS-P, with difficulty identifying and describing emotions related to negative urgency. Consistent with the findings of Fink et al. (2010) and Pike (2013), this suggests that individuals who have increased difficulty recognising and expressing their emotions may be more likely to act rashly in response to negative affect.

When exploring impulsivity via a behavioural measure, no significant correlations were found between delay discounting and study variables, including BMI. This is consistent with previous research by Nederkoorn et al. (2006), Yeomans et al. (2008) and Rasmussen et al. (2010), who found a similar pattern of discounting in individuals with a healthy BMI. It is possible that similar findings have been found due to a predominantly female sample (90.67\%), as previous studies have been conducted in female only samples. However, Weller et al. (2008) found a significant difference in discounting behaviours between individuals with and without obesity in females. A significant difference was also found by Davis et al. (2010) between a 
healthy and clinical sample of females. Participants in the current study were much younger and had a narrower age range than Davis et al. (2010) which could offer an explanation for the contrasting findings. To note, it is unlikely the contrasting results are due to the magnitude and delay of the rewards, as across the literature, studies have used short (one year) to long (25 years) delays and small (£100) to large $(£ 50,000)$ values of money and these have all contributed to mixed findings. Delay discounting was not significantly correlated with emotional eating, alexithymia or negative urgency. A meta-analysis also found no significant association between negative urgency and delay discounting (Cyders \& Coskunpinar, 2011b), highlighting that the current findings are in line with expectations. There does not appear to be any previous literature exploring the relationship between delay discounting and alexithymia, and therefore, this study is the first to explore these associations.

The second aim of the study was to comprehensively test the proposed theoretical model. The model incorporated negative affect (depression and anxiety) and negative urgency as possible mediating factors in the relationship between alexithymia and emotional eating (see Figure 1). Four main findings emerged. First, total alexithymia scores were indirectly associated with emotional eating via both negative urgency alone and via negative affect and negative urgency (similar pattern of results for depression and anxiety scores), with no significant difference in the strength of the two specific indirect effects. This suggests that increased alexithymia may result in an individual experiencing greater negative affect, leading them to react rashly to alleviate the associated sensations, and in turn exacerbates the tendency to emotional eat. Second, the DIF subscale was significantly and indirectly associated with emotional eating via negative urgency only, indicating that when individuals are unable to identify their emotions, they may act impulsively to alleviate these unknown 
feelings (negative urgency) and engage in emotional eating in response. Third, negative affect (similar patterns found for both depression and anxiety) and negative urgency significantly mediated the indirect relationship between DDF and emotional eating. Therefore, when an individual experiences difficulty describing emotions, they may be more vulnerable to experiencing depression and anxiety, and in turn, may act rashly in response and engage in emotional eating. Finally, the EOT subscale of the TAS-20 did not have any significant direct or indirect relationships with emotional eating as measured by both EES and the TFEQ.

The third aim was to expand the theoretical model of emotional eating to explain variance in BMI. When negative affect was defined as depression, a significant direct effect of difficulty identifying feelings on BMI was found. Furthermore, a specific indirect effect via depression and negative urgency was also found. Therefore, difficulty identifying emotions may increase vulnerability to depression and lead to a greater tendency to react rashly to alleviate associated sensations (negative urgency), thereby increasing the likelihood of engaging in emotional eating, and in turn, increased BMI. There were no significant direct or indirect effects for total alexithymia scores or the DDF and EOT subscales on BMI. When anxiety scores were included in the models, an indirect effect via negative urgency and emotional eating was found for total alexithymia scores and the difficulty identifying subscale, but only for emotional eating as measured by the TFEQ. The role of difficulty describing feelings appears to be stronger when anxiety is included in the model as three significant indirect effects were found. These effects were through the following pathways: firstly, via increased anxiety scores only; secondly, via increased negative urgency and emotional eating, and lastly, via increased anxiety, negative urgency and emotional eating. Contrast analysis revealed that the indirect pathway via increased anxiety scores was the 
strongest, suggesting that when individuals experience increased difficulty describing feelings, they are more prone to increased levels of anxiety, leading to increased BMI.

The large amount of delay discounting data removed because of nonsystematic responding represents a limitation of the current study. To avoid being too stringent, a $25 \%$ threshold was used for criteria 1 and $5 \%$ for criteria 2 taking into consideration LLR amount (£100) and maximum delay (one year). Previously, 20\% and $10 \%$ have been used respectively, but this was for a much larger LLR and over a much longer time period (£1000 and 25 years; Johnson \& Bickel, 2008). Johnson and Bickel (2008) suggested that such responding could be due to participants not understanding the task, a lack of attention, experimental instructions not encouraging careful responding, or idiosyncratic behaviour (e.g. a participant completing the task near a time when their rent was due to be paid, increasing their awareness of needing money sooner). In the current study, participants were given instructions on the computer screen and completed a practice trial. Furthermore, the computer programme used had a double limit procedure built in to ensure that a single answer could not influence the convergence on the IP. In theory, this should have reduced the likelihood of a criteria 1 bias occurring. However, as the practice trial did not present instances of all the possible delays, participants may have initially responded to how they first interpreted the LLR and SSR, only considering the trade-off between delay and reward as the DD task progressed. In line with this, non-systematic responders reported lower income compared to systematic responders and a difference in age between responder groups approached significance. Both receiving a lower income and being younger have been found to be related to greater impulsivity (Green et al., 1994, 1996, 1999; McCrae et al., 1999), and therefore, it may be that non-systematic responders did not fully consider how the task would progress or how they would respond to other delays, 
resulting in at least one IP that was less by a minimum of $£ 25$ at a smaller delay than a later delay (e.g. selecting $£ 30$ as the SSR at 2 days but $£ 60$ as the SSR at 1 week over $£ 100$ at each delay respectively). However, lower income in the non-responders is counterintuitive as individuals with a higher income could be expected to be more inclined to take the larger, later reward. Indeed, if money was not a concern in the present for such participants, choosing to opt for the larger later reward after a year delay would not be surprising. In contrast, in the current study it was individuals with a lower income who selected a similar larger later reward after a one-day one-year delay. It may be that despite having a lower income, these participants had a sufficient income to survive and therefore viewed the task as 'bonus' money and were always willing to wait as a result. Alternatively, these individuals may have been very low in impulsiveness in general. A large percentage of participants (20\%) were removed for criteria 2 and it is possible that 'floor' effects may have occurred. This is when discounting is shallow and is evident when participants choose a similar SSR after one day and one-year. It could be argued that one year is a relatively small time frame, and therefore, it may be valid for an individual to discount by less than $5 \%$ from the first IP to the last. However, as the LLR was also relatively small (£100) and the magnitude effect (the inverse relationship between rate of discounting and magnitude of reinforcer; Mellis, Woodford, Stein, \& Bickel, 2017) is likely to be balanced; $£ 100$ across one year has been proposed as an appropriate LLR and delay (Davis et al., 2010; Price, 2016).

\subsubsection{Study One: Key Findings}

- Difficulty identifying feelings had a significant direct effect on BMI and a significant specific indirect effect via negative urgency and emotional eating (when depression was included in the model). 
- A significant indirect effect via negative urgency and emotional eating was found for total alexithymia and difficulty identifying feelings subscale scores on BMI (when depression was included in the model).

- The role of difficulty describing feelings appeared to be stronger when anxiety was included in the model as three significant indirect specific effects were found: via anxiety only; via negative urgency and emotional eating, and via anxiety, negative urgency and emotional eating.

\subsection{Study Two: Aims}

Study Two aimed to re-examine the relationships between alexithymia, negative affect, negative urgency, emotional eating and BMI. Consistent with the results of Study One, significant positive correlations between alexithymia, negative affect, negative urgency and emotional eating were predicted. As BMI did not significantly correlate with any other variables in Study One, the relationship between BMI, alexithymia, negative affect, negative urgency and emotional eating was further examined in Study Two.

To ensure the theoretical models proposed in Study One were developed in a robust manner, a self-replication was performed to test the applicability of the model in a more representative general population sample. Consistent with the findings from Study One, negative affect and negative urgency were expected to play a significant mediating role between alexithymia and emotional eating. In addition, similar indirect and direct effects between DIF and BMI were expected to emerge when depression was included in the model. When anxiety was included in the model, it was expected that DDF would have a significant indirect effect on BMI via anxiety alone, via 
negative urgency and emotional eating, and via anxiety, negative urgency and emotional eating.

\subsection{Study Two: Methods}

\subsubsection{Participants}

The study was advertised on social media and via Swansea University's media platforms. To be eligible to take part in the study, participants had to be between 18 65 years of age (in line with exclusion criteria as stipulated in section 2.3.1.) and report no history of eating, substance use or addictive disorders. 651 participants accessed the survey, although data for 309 participants was subsequently removed for the reasons outlined in Table 6.

Of the remaining 342 participants, $279(81.6 \%)$ were female and mean age was 32.36 years $(S D=11.38$, range $=18.20-64.13$ years $)$. The majority of participants $(n$ $=315 ; 92.1 \%)$ reported their ethnicity as White. Other ethnicities reported were Asian $(n=7 ; 2 \%)$, Black $(n=3 ; .9 \%)$, Chinese $(n=2 ; .6 \%)$, Mixed $(n=8 ; 2.3 \%)$ and seven (2\%) preferred not to say or selected other. The majority of participants were either single $(n=150 ; 43.9 \%)$ or married $(n=109 ; 31.9 \%)$, with remaining participants reporting themselves as either cohabiting $(n=65 ; 19 \%)$, divorced $(n=14 ; 4.1 \%)$, separated or widowed $(n=4 ; 1.2 \%)$. A large proportion of participants were in full time employment $(n=153 ; 44.7 \%)$ or were students $(n=100 ; 29.2 \%)$, with others reporting themselves as being in part time employment $(n=61 ; 17.8 \%)$, unemployed $(n=11 ; 32 \%)$, self-employed $(n=7 ; 2.0 \%)$ or retired or volunteering $(n=10 ; 3 \%)$. The sample had a mean self-reported BMI of $25.94(S D=5.51$, range $=16.33-48.10)$. 
Average weekly alcohol intake was 6.02 units $(S D=.7 .75$, range $=0-50$ units $)$ and 30 participants $(8.8 \%)$ reported being regular smokers.

An a priori power analysis (G*Power; Faul et al., 2007) indicated that a minimum sample size of 84 participants was required (effect size $=.25$ - medium; power $=.8)$. Therefore, the final sample size $(N=342)$ was sufficient to ensure adequate statistical power.

Table 6. Reasons for participant exclusions in Study Two.

\begin{tabular}{ll}
\hline Reason & Number \\
\hline History of eating disorder & 36 \\
History of substance use or addictive disorder & 3 \\
Uncomplete consent & 10 \\
Accessed the survey but did not complete & 239 \\
Outliers (3 SD over the mean) & \\
a) BMI & 5 \\
b) BDI & 4 \\
c) BAI & 4 \\
Inaccurate reporting (e.g. BMI = 9.26) & 1 \\
BMI < 16 & 6 \\
Age $>65$ years & 1 \\
\hline
\end{tabular}

\subsubsection{Measures}

\subsubsection{Demographics}

The following information was obtained for all participants where available: date of birth, sex, marital/relationship status, employment status and ethnicity. All participants were asked if they had a history eating, addictive or substance use disorders. 


\subsubsection{Body Mass Index (BMI)}

Participants were asked to report their weight $(\mathrm{kg})$ and height $(\mathrm{m})$ to allow BMI to be calculated. Self-reported weight and height data has been used in a number of studies involving general population samples, with good levels of consistency between self-reported and recorded BMI values in the laboratory reported (Jeffery, 1996; Kuczmarski, Kuczmarski, \& Najjar, 2001).

\subsubsection{Positive Affect and Negative Affect Scale}

(PANAS; Watson et al., 1988). As fully described in Chapter Two (section 2.3.2.2.). In line with Study One, high levels of internal consistency were found (PA: $\alpha=.89$ and NA: $\alpha=.87)$.

\subsubsection{Toronto Alexithymia Scale}

(TAS-20; Bagby, Parker, et al., 1994; Bagby, Taylor, et al., 1994). As fully described in Chapter Two (section 2.3.2.3.). Cronbach's alphas were as follows; total TAS-20: $\alpha=.88$, DIF: $\alpha=.87$ DDF: $\alpha=.83$, and EOT: $\alpha=65$.

\subsubsection{UPPS-P}

(Cyders et al., 2007; Whiteside \& Lynam, 2001). As fully described in Chapter Two (section 2.3.2.4.) Levels of internal consistency were found to be acceptable to good in the current sample (NU: $\alpha=.80$; PU: $\alpha=.85$; Prem: $\alpha=.75$; Pers: $\alpha=.82$; SS: $\alpha=.85 ;$ and total UPPS-P: $\alpha=.90$ ).

\subsubsection{Beck Depression Inventory}

(BDI; Beck et al., 1996; Beck, Steer, et al., 1988). As fully described in Chapter Two (section 2.3.2.5.). As with Study One, internal consistency of the measure was excellent, $\alpha=.93$. 


\subsubsection{Beck Anxiety Inventory}

(BAI; Beck, Epstein, et al., 1988; Beck \& Steer, 1990). As fully described in Chapter Two (section 2.3.2.6.). The BAI exhibited an excellent level of internal consistency in the current study $(\alpha=.91)$.

\subsubsection{Emotional Eating Scale}

(EES; Arnow et al., 1995). As fully described in Chapter Two (section 2.3.2.7.). Good to excellent levels of internal consistency were found; depression: $\alpha=$ .81 ; anger/frustration: $\alpha=.93$; and anxious: $\alpha=.86$; and total EES: $\alpha=.95$.

\subsubsection{Three Factor Eating Questionnaire - Revised}

(TFEQ; Karlsson et al., 2000). As fully described in Chapter Two (section 2.3.2.8.). Similar levels of internal consistency were found as in Study One: cognitive restraint, $\alpha=.78$; uncontrolled eating, $\alpha=.89$, and emotional eating, $\alpha=.82$. Based on research highlighting that the smell of bacon cooking also evokes the urge to eat in vegetarians (Childers \& Herzog, 2009), one item from the uncontrolled eating subscale was amended to be more inclusive of vegetarians. The item was changed to "When I smell sizzling bacon or steak I find it very difficult to keep from eating, even if I have just finished a meal," from previously just stating "sizzling steak".

\subsubsection{Procedure}

Ethical approval was obtained from the Department of Psychology Ethics Committee, College of Human and Health Sciences, Swansea University. The study was advertised on social media sites (e.g. Facebook, Twitter) and on flyers displaying a Q-code as a link to information about the study. The study was advertised as "An investigation into the relationship between alexithymia, impulsivity, and eating 
behaviours". It was described as an exploration of the relationship between our ability to describe and identify emotions, our levels of impulsivity, and how we regulate our eating behaviours. Participants completed the study online via Survey Monkey (Palo Alto, California, USA) in their own time. Participants were presented with an information page and upon providing full consent, were asked to answer standard demographic questions followed by the PANAS, TAS-20, BDI, BAI, UPPS-P, EES and TFEQ. Participants were also asked for their height and weight and were provided with conversion details to report both in metric format. If participants wanted to withdraw at any time, they could do so by closing the web browser. Upon completion, participants were presented with a debrief page and were thanked for their time.

\subsubsection{Statistical Analysis}

Statistical analysis was carried out using SPPS 22.0 and PROCESS 2.16.3. As all variables were normally distributed, Pearson's correlation analysis was performed.

\subsubsection{Serial Mediation Model}

A series of serial multiple mediation models (Hayes, 2013) were conducted (see Table 1). The first 16 models entered alexithymia total or subscale scores as the predictor variable $(X)$ and emotional eating as measured by either the EES or TFEQ as the outcome variable $(Y)$. Negative affect (separate models for depression and anxiety) was entered as the mediating factor at $M_{1}$ and negative urgency was entered at $M_{3}$. In a final set of 16 models (see Table 1), self-reported BMI was entered as an outcome variable $(Y)$ with emotional eating (EES/TFEQ) entered as a mediator at $M_{2}$, in addition to negative affect (anxiety/depression) at $M_{1}$ and negative urgency at $M_{2}$. Self- 
reported BMI (models 1-16 only), age, gender, uncontrolled eating and cognitive restraint scores were controlled for in all models.

\subsection{Study Two: Results}

\subsubsection{Descriptive Statistics}

Descriptive statistics for Study Two can be found in Table 7.

\subsubsection{Correlational Analysis}

2.8.2.1. Correlations between alexithymia, negative urgency, negative affect, emotional eating and BMI

Pearson's correlations showed that TAS-20 total, DIF and DDF scores were significantly, positively correlated with emotional eating as measured by the EES and the TFEQ (see Table 8). Negative urgency was positively significantly correlated with emotional eating; EES: $r=.350, p<.001$ and TFEQ: $r=.324, p<.001$. There were also positive, significant correlations between BDI and emotional eating scores as measured by both the EES and TFEQ (see Table 8). BAI scores were only significantly correlated to EES total scores. BMI scores were not significantly correlated to alexithymia but were weakly correlated with negative urgency $(r=.152, p=.005)$ and emotional eating (EES: $r=.212, p<.001$, TFEQ: $r=.2, p<.001$ ). Correlations between the subscales of all variables are presented in Appendix L. 
Table 7. Mean, standard deviation and range for each study variable in Study Two.

\begin{tabular}{|c|c|c|c|}
\hline Measure & Mean & $\mathrm{SD}$ & Range \\
\hline PA & 32.11 & 7.88 & $13.00-49.00$ \\
\hline NA & 20.94 & 7.44 & $10.00-50.00$ \\
\hline DIF & 15.27 & 6.58 & $7.00-33.00$ \\
\hline DDF & 12.73 & 4.98 & $5.00-23.00$ \\
\hline EOT & 18.84 & 4.87 & $8.00-32.00$ \\
\hline TAS-20 & 46.84 & 13.33 & $22.00-82.00$ \\
\hline $\mathrm{NU}$ & 29.42 & 7.44 & $15.00-45.00$ \\
\hline PU & 26.61 & 7.16 & $13.00-48.00$ \\
\hline Prem & 23.16 & 5.46 & $11.00-41.00$ \\
\hline Pers & 20.53 & 4.98 & $10.00-34.00$ \\
\hline SS & 30.61 & 6.86 & $13.00-48.00$ \\
\hline UPPS-P & 130.34 & 21.50 & $72.00-206.00$ \\
\hline BDI & 12.71 & 10.77 & $.00-48.00$ \\
\hline BAI & 10.66 & 9.77 & $.00-43.00$ \\
\hline Ang/Frus & 20.51 & 9.53 & $10.00-55.00$ \\
\hline Anx & 16.93 & 6.96 & $9.00-45.00$ \\
\hline Dep & 12.11 & 4.93 & $4.00-24.00$ \\
\hline EES & 50.88 & 19.79 & $24.00-125.00$ \\
\hline TFEQ-CR & 52.59 & 16.50 & $21.43-96.43$ \\
\hline TFEQ-UE & 58.63 & 17.96 & $25.00-97.22$ \\
\hline TFEQ-EE & 57.65 & 22.31 & $25.00-100.00$ \\
\hline
\end{tabular}

Ang/Frus $=$ anger/frustration EES subscale, Anx $=$ anxiety EES subscale, $\mathrm{BAI}=$ Beck anxiety inventory BDI $=$ Beck depression inventory, DDF $=$ difficulty describing feelings, Dep = depression EES subscale, DIF = difficulty identifying feelings, EOT $=$ externally oriented thinking, $\mathrm{EES}=$ total EES, $\mathrm{NA}=$ negative affect, $\mathrm{NU}=$ negative urgency, $\mathrm{PA}=$ positive affect, Pers $=$ (lack of) perseverance, Prem $=$ (lack of $)$ premeditation, $\mathrm{PU}=$ positive urgency, $\mathrm{SS}=$ sensation seeking, TAS-20 = total Toronto alexithymia scale, TFEQ-CR = cognitive restraint TFEQ subscale, TFEQ-EE = emotional eating TFEQ subscale, TFEQ-UE = uncontrolled eating TFEQ subscale, UPPS-P = total UPPS-P. 
Table 8. Pearson correlations between alexithymia, negative urgency, negative affect, emotional eating and B

\begin{tabular}{|c|c|c|c|c|c|c|}
\hline & 1. & 2. & 3. & 4. & 5. & 6. \\
\hline 1. DIF & - & & & & & \\
\hline 2. $\mathrm{DDF}$ & $.706 * * *$ & - & & & & \\
\hline 3. EOT & $.275 * * *$ & $.453 * * *$ & - & & & \\
\hline 4. TAS-20 & $.858 * * *$ & $.887 * * *$ & $.670 * * *$ & - & & \\
\hline 5. $\mathrm{NU}$ & $.524 * * *$ & $.396 * * *$ & $.214 * * *$ & $.485 * * *$ & - & \\
\hline 6. BDI & $.645 * * *$ & $.516^{* * *}$ & $.213 * * *$ & $.589 * * *$ & $.544 * * *$ & - \\
\hline 7. BAI & $.553 * * *$ & $.396 * *$ & $.118 *$ & $.464 * * *$ & $.354 * * *$ & $.699 * * *$ \\
\hline 8. EES & $.265 * * *$ & $.174 * * *$ & .058 & $.217 * *$ & $.350 * * *$ & $.317 * * *$ \\
\hline 9. TFEQ-EE & $.180 * * *$ & $.218^{*}$ & -.004 & $.135^{*}$ & $.324 * * *$ & $.219 * * *$ \\
\hline 10. BMI & .050 & -.025 & -.002 & .016 & $.152 * *$ & $.130 *$ \\
\hline
\end{tabular}

BAI = Beck anxiety inventory, BDI = Beck depression inventory, BMI = body mass index, DDF = difficulty identifying feelings, EES = total EES, EOT = externally oriented thinking, NU = negative urgency, TAS-20 $\mathrm{EE}=$ emotional eating TFEQ subscale. $*=p<.05, * *=p<.01, * * *=p<.001$. 


\subsubsection{Serial multiple mediation models for emotional eating}

Models 1-4 revealed no significant direct or indirect effects were found when TFEQ was entered as $Y$.

Model 5 entered total alexithymia scores as the predictor variable $(X)$ and used emotional eating as measured by the EES as the outcome variable $(Y)$. No significant direct effect was found but the specific indirect effect via BDI $\left(a_{1} b_{1}\right)$ was significant, $B=.1267, C I=.0400-.2238$. The total indirect effect $(a b)$ for this model was also significant, $B=.1277, C I=.0331-.2288$.

Models 6-8 entered the TAS-20 subscales at $X$. Results followed a similar pattern to Model 5 with no significant direct effect but a significant specific indirect effect via BDI $\left(a_{1} b_{1}\right.$; Model 6 - DIF: $B=.2680, C I=.0655-.4943$; Model 7 - DDF: $B$ $=.2898, C I=.0970-.5227$ and Model $8-$ EOT: $B=.1081, C I=.0340-.2368)$. All three models also had significant total indirect effects ( $a b$; DIF: $B=.2653, C I=.0337-.4989$; DDF: $B=.2924, C I=.0970-.5169$; EOT: $B=.1133, C I=.0276-.2389)$.

Models 9-12 replaced BDI with BAI at $M_{1}$ and used emotional eating as measured by the TFEQ as the outcome variable $(Y)$. Similar to Models 1-4, no significant direct or indirect effects were found.

Model 13 entered EES at $Y$. There was no significant direct effect between total alexithymia scores and emotional eating, but there was a significant specific indirect effect via BAI $\left(a_{1} b_{1}\right), B=.0740, C I=.0144-.1452$. The total indirect effect $(a b)$ was also significant, $B=.0935, C I=.0060-.1871$.

Models 14-16 entered the DIF, DDF and EOT as $X$ respectively. No significant direct or indirect effects were found in Model 16 (EOT as $X$ ). Model 14 (DIF as $X$ ) and 15 (DDF as $X)$ found a significant specific indirect effect via BAI $\left(a_{1} b_{1}\right.$; Model 14: $B$ 
$=.1710, \mathrm{CI}=.0117-.3574 ;$ Model 15: $B=.1635, C I=.0394-.3150)$. Model 15 also had a significant total indirect effect $(a b) B=.2078, C I=.0374-.3982$.

\subsubsection{Serial multiple mediation Models for BMI}

Model 17 entered BDI at $M_{2}$ and emotional eating as measured by TFEQ at $M_{3}$ and no significant direct effect between total alexithymia scores $(X)$ and BMI $(Y)$ was found. However, a significant specific indirect effect via BDI was found $\left(a_{1} b_{2}\right), B=$ $.0335, C I=.0019-.0660$. The total indirect effect was also significant $(a b), B=.0422$, $C I=.0087-.0781$

Model 18-20 found no significant direct effects. All models found a significant indirect effect via BDI $\left(a_{1} b_{1}\right)$; Model 18 - DIF as $X: B=.0795, C I=.0103-.1531$; Model 19 - DDF as $X: B=.0786, C I=.0097-.1518$, and Model $20-$ EOT as $X: B=$ $.0214, C I=.0008-.0609$. A significant total indirect effect $(a b)$ was also found for DIF (Model 18), $B=.1010, C I=.0283-.1840$, and DDF (Model 19), $B=.1010, C I=.0316-$ .1768 .

Model 21 entered EES at $M_{3}$ and found no significant direct effect between total alexithymia scores and BMI. There was a significant specific indirect effect via BDI $\left(a_{1} b_{2}\right), B=.0317, C I=.0008-.0636$ and a significant total indirect effect $(a b), B$ $=.0451, C I=.0125-.0801$.

Model 22-24 entered the TAS-20 alexithymia subscales at $X$. No significant direct effects were found and no specific indirect effect for EOT was found in Model 24. DIF (Model 22) and DDF (Model 23) as $X$ had one significant indirect effect via BDI $\left(a_{1} b_{1}\right.$; DIF: $B=.0756, C I=.0086-.1462$ and DDF: $\left.B=.0734, C I=.0047-.1442\right)$. All models had a significant total indirect effect ( $a b$; Model 22 - DIF: $B=.1063, C I=$ 
$.0337-.1878$; Model 23 - DDF: $B=.1023, C I=.0325-.1804$ and Model $24-$ EOT: $B=$ $.0287, C I=.0021-.0678)$.

Model 25 entered BAI at $M_{1}$ and emotional eating as measured by the TFEQ at $M_{3}$. Analysis revealed no significant direct effect between total alexithymia scores and BMI. There was a specific indirect effect via BAI and NU $\left(a_{1} d_{21} b_{2}\right), B=.0021, C I=$ $.0001-.0066$.

Model 26-28 entered the TAS-20 alexithymia subscales at $X$. No direct or indirect effects were found in Model 26 (DIF as $X$ ). Model 27 (DDF as $X$ ) had no significant direct effect, but two significant specific indirect effects were found. The first via NU $\left(a_{2} b_{2}\right), B=.0341, C I=.0015-.0788$ and the second was via BAI and NU $\left(a_{1} d_{21} b_{2}\right), B=.0072, C I=.0008-.0189$. Contrast analysis revealed that the specific indirect effect via NU was significantly stronger than the indirect effect via BAI and NU, $B=-.0269, C I=-.0698--.0021$. Model 28 (EOT as $X)$ also had no significant direct effect, but there was a significant specific indirect effect via NU $\left(a_{2} b_{2}\right), B=$ $.0163, C I=.0006-.0449$.

Model 29 entered emotional eating as measured by EES at $M_{3}$, and no significant direct effect between total alexithymia scores and BMI was found. There was one significant indirect effect via BAI and NU $\left(a_{1} d_{21} b_{2}\right), B=.0021, C I=.0001$ .0009 , and a second one approached significance via BAI, NU and TFEQ-EE $\left(a_{1} b_{21} b_{32} d_{3}\right), B=.0001, C I=.0000-.0011$.

Model 30-32 entered the TAS-20 subscales at $X$. There were no significant direct or indirect effects between DIF and BMI (Model 30). For Model 31 (DDF as $X$ ), two significant specific indirect effects were found. The first via NU $\left(a_{2} b_{2}\right), B-.0335$, $C I=.0013-.0770$ and the second via BAI and NU $\left(a_{1} d_{21} b_{2}\right), B=.0070, C I=.0007-$ .0187. Contrast analysis revealed that the indirect effect via NU was significantly 
stronger than the indirect effect via BAI and NU, $B=-.0264, C I=-.0686--.0015$. No significant direct effect was found in Model 32 (EOT as $X$ ), but there was a significant specific indirect effect via NU $\left(a_{2} b_{2}\right), B=.0161, C \mathrm{I}=.0006-.0464$, and a second via BAI, NU and EES $\left(a_{1} b_{21} b_{32} d_{3}\right), B=.0001, C I=.0000-.0013$ that approached significance.

\subsection{Study Two: Discussion}

Study Two aimed to confirm the associations between alexithymia, emotional eating, negative affect and negative urgency, with results generally consistent with the findings of Study One and previous research (Larsen et al., 2006; Ouwens et al., 2009; Pike, 2013; van Strien et al., 2016). However, in contrast to the findings of Study One, BMI (laboratory measured in Study one versus self-reported in Study Two) was significantly and positively related to emotional eating, suggesting that higher levels of self-reported emotional eating are associated with higher self-reported BMI scores (in line with previous studies - Blair et al., 1990; Geliebter \& Aversa, 2003; Koenders \& van Strien, 2011; van Strien, Herman, et al., 2012). Emotional eating is thought to involve the overconsumption of 'comfort' food which tends to be high in fat and sugar (e.g. Torres \& Nowson, 2007; Wardle, Steptoe, Oliver, \& Lipsey, 2000), which could help explain the association between greater engagement in emotional eating and higher BMI in the current study.

Study Two also aimed to examine the theoretical models proposed in Study One in a robust manner through self-replication in a more representative general population sample. Depression and anxiety significantly mediated the relationship between total alexithymia and subscale scores and emotional eating; however, there 
was no significant indirect effect of BAI for EOT. Such findings suggest that increased difficulty understanding emotions may increase an individual's vulnerability to experiencing anxiety and depression, resulting in increased engagement in emotional eating.

Although, the models were only significant when emotional eating was measured by the EES. The EES and TFEQ were moderately correlated with one another $(r=.61, p<.001)$, but their relationship with measures of anxiety and depression differed. For example, the relationship between depression and emotional eating was much weaker when emotional eating was measured by the TFEQ. Similarly, a significant relationship between emotional eating and anxiety was only found between the EES and BAI. In part, this may be due to varying items across the two emotional eating measures, indicating differences in the way emotional eating is conceptualised and assessed. For example, the EES consists of a wide range of emotions whereas the TFEQ consists of just three ("blue; lonely; anxious"). Therefore, it may be that the associations between alexithymia and emotional eating are only present across a broader range of emotions. Indeed, individuals with alexithymia may be able to understand more 'simple' emotions (e.g. blue, lonely, anxious) but have difficulty when they become more complex. Therefore, individuals with alexithymia may not experience as much difficulty identifying and understanding the emotions captured in the TFEQ, whereas the more complex emotions within the EES, such as "on edge", "inadequate", "resentful" and "confused" may lead to greater difficulties. In turn, such difficulties could increase one's propensity to engage in emotional eating. The theoretical models were also expanded to explain variance in BMI, with two main pathways identified. When negative affect was defined as depression, it significantly mediated the relationship between total and subscale scores of 
alexithymia and BMI. Therefore, this suggests that increased difficulty understanding emotions may lead to an increased tendency to experience depressive symptoms, which then leads to an increase in BMI. In contrast, when negative affect was defined as anxiety, anxiety and negative urgency in serial significantly mediated the pathway between DDF, externally oriented thinking and BMI. Here, difficulty describing emotions may lead to an increased tendency to experience negative affect in the form of anxiety, with an individual acting rashly in response to avoid these sensations; resulting in increased BMI.

\subsubsection{Study Two: Key Findings}

- A significant indirect effect was found between alexithymia and emotional eating (as measured by the EES) via negative affect (both depression and anxiety in separate models).

- All aspects of alexithymia (DIF, DDF and EOT) were significantly and indirectly related to emotional eating via depression scores.

- When anxiety was included in the model, there were no significant direct or indirect effects of DIF on emotional eating.

- Two indirect effects were found for total alexithymia, DDF and EOT scores on emotional eating. These indirect effects were either via NU or via BAI and NU.

\subsection{Chapter Discussion}

Similar associations between alexithymia, negative affect, impulsivity, and emotional eating were found across Studies One and Two. In contrast, the relationship between BMI and emotional eating differed across studies. Namely, no significant 
relationship between BMI and emotional eating was found in the student sample, but a significant positive relationship was found in the general population sample. As previously mentioned, contrasting results have also been reported in the literature. For example, Koenders \& van Strien (2011) and van Strien, Herman, et al. (2012) reported a significant positive relationship between emotional eating and BMI, whereas Adriaanse et al. (2011) and Snoek, van Strien, et al. (2007) found no such relationship. One possible explanation is the different methods used for measuring BMI. In Study One, participants had their height and weight recorded in the laboratory, whereas participants in Study Two self-reported their height and weight. However, even though some studies suggest that self-report methods can lead to an underestimation and overestimation of weight and height respectively (Spencer, Appleby, Davey, \& Key, 2002), self-reported body weight has been found to be an excellent approximation of actual body weight across a broad range of populations (Jeffery, 1996; Kuczmarski et al., 2001). Therefore, it is unlikely that the difference in findings observed across studies is due to methodological differences in their collection of BMI data.

A second possible explanation for such contrasting findings may rest in the composition of samples across studies. Notably, the sample in Study Two had a broader age range (18-64 years) and a slightly higher BMI $(M=25.94)$ overall compared to the sample in Study One (18-36 years; BMI: $M=23.99)$. Different selfreport measures of emotional eating are also important to consider. In the current studies, EES and TFEQ were used, whereas commonly DEBQ is used. Price et al. (2015) found that TFEQ and DEBQ emotional eating subscales were significantly, although weakly, correlated with BMI but the subscales of the EES were not. Whilst Price et al. (2015) did not explore a total EES score; it nevertheless highlights how the strength of relationship between BMI and emotional eating differs depending on what 
measure of emotional eating is employed. Furthermore, the validity of self-report measures of emotional eating has been questioned (Domoff, Meers, Koball, \& Musher-Eizenman, 2014). Evers et al. (2009), who used only the DEBQ in their study, noted potential issues with validity as a result. However, even though two measures of emotional eating (TFEQ and EES) were used here; concurrent validity could still be an issue. Indeed, this seems likely as even though a moderate relationship was observed between the two measures of emotional eating, their relationship with BMI ultimately differed.

An additional aim of both studies was to further explore the role of emotional dysregulation, and specifically alexithymia, as a mechanism underpinning emotional eating. Guided by previous literature, a novel theoretical model was proposed that suggested alexithymia was indirectly associated with emotional eating through negative affect (anxiety and depression examined separately) and impulsivity (negative urgency; see Figure 1, section 1.9). Specifically, it was proposed that alexithymia would be associated with greater levels of negative affect, leading individuals to act rashly in response (negative urgency) to avoid the associated sensations and to engage in emotional eating. Initially the model was tested in a student sample (Study One) before completing a self-replication in a more general population sample (Study Two).

One main difference between the two samples was the role of negative urgency. In Study One, the relationship between alexithymia and emotional eating was significantly mediated by both negative affect and negative urgency either individually or in a serial pathway. In contrast, in Study Two negative affect (depression and anxiety) was the only significant indirect effect. There is no clear reason why the models in the two samples differ. For example, there were similar levels of negative 
urgency, depression and anxiety across both samples. Similar levels were also found for emotional eating as measured by the EES, but TFEQ scores were slightly higher in the more general population sample (Study One: $M=42.58, S D=29.15$, Study Two: $M=50.88, S D=19.79)$. However, this does not lend itself as an explanation as within Study Two, no significant direct or indirect effects were found for TFEQ-EE.

The next step was to expand the theoretical model to explain variance in BMI. Three main findings emerged from the studies. First, it appears that the pathways between alexithymia and BMI seem to differ depending on whether negative affect is represented by anxiety or depression. This is not entirely surprising, as even though alexithymia is related to negative affect overall (Suslow \& Donges, 2017), its relationship with anxiety and depression is thought to differ (Hendryx et al., 1991; Marchesi et al., 2000). For example, Eizaguirre et al. (2004) found that depression was a significant predictor of alexithymia total and subscale scores in individuals with eating disorders, whereas anxiety was only a predictor of total alexithymia and DIF subscale scores when combined with depression. This indicates that depression may have stronger associations with alexithymia, which is supported by the current findings and is consistent with the view that depression and alexithymia may be overlapping constructs (Corcos et al., 2000; Honkalampi, Hintikka, Laukkanen, \& Viinamäki, 2001; Marchesi et al., 2000; Parker, Bagby, \& Taylor, 1991). In support of this argument, Torres and colleagues (2015) previously highlighted how both constructs share multiple characteristics, including negative affect (Mattila, Poutanen, Koivisto, Salokangas, \& Joukamaa, 2008), decreased ability to communicate affect to other people (Saarijärvi, Salminen, \& Toikka, 2001), problems with interpersonal communication (Mattila et al., 2008), and lack of emotional clarity (Rude \& McCarthy, 2003). 
The current findings also reinforce the view that depression and anxiety should always be considered as distinct emotional states within eating behaviour research. Goossens et al. (2009) argued that eating in response to anxiety reduces hyper arousal, whereas eating in response to depression increases positive mood. Studies have also suggested that emotional or binge eating may serve to reduce anxiety but may increase depression (Haedt-Matt \& Keel, 2011; Rosenbaum \& White, 2015), and Finch and Tomiyama (2015) found that emotional eating acted as a buffer to daily life stresses in women, but only when they did not have elevated depressive symptoms.

Second, different facets of alexithymia produced different pathways. For example, the DIF subscale of the TAS-20 directly or indirectly predicted BMI in the majority of models. Indeed, the only time that it did not play a role was when anxiety was included in the general population sample. Overall, these findings suggest that difficulty identifying feelings may be a core driving feature of alexithymia relevant to emotional eating, and in turn, BMI. In support of this, Pinaquy et al. (2003) previously found that DIF was the strongest predictor of emotional eating, and Larsen et al. (2006) also found that DIF significantly mediated the relationship between depression and emotional eating alongside impulsivity.

Third, different pathways between alexithymia and BMI in the student versus general population sample were found. This finding does not seem to be attributable to demographic differences across the two samples. For example, even though age, as well as levels of cognitive restraint and uncontrolled eating differed across the samples, these were controlled for in the analyses. Although, it is possible that other demographic differences may have existed across the samples. For example, it seems likely that years spent in education and socioeconomic status would have differed across the two samples; factors known to be associated with both alexithymia and the 
adoption of emotional regulation strategies (Lane et al., 1998; Parker et al., 2001). Different pathways across the two samples highlight important methodological considerations in the development of models. Behaviour research using human participants relies heavily on convenient student samples, but results may only be applicable to other student samples and may not generalise to a broader population. For this reason, completing a self-replication, as done here, should be seen as a pivotal step in similar research in the future. Therefore, although contrasting results were found, it nevertheless highlights how important it is that initial models are interpreted with caution until they undergo rigorous testing in broader samples.

Studies One and Two are not without limitations. First, the samples consisted predominantly of females. This may have influenced results as males are reported to exhibit greater levels of alexithymia than their female counterparts (Honkalampi et al., 2000; Mattila et al., 2006), as well as lower levels of depression and emotional eating (e.g. Larsen et al., 2006). Therefore, it is possible that the pathways between alexithymia, emotional eating and BMI may differ across genders and this should be explored in future research. Second, even though age was controlled for in the analyses, additional age-related factors were not, such as the use of more adaptive and less impulsive coping strategies over the course of one's lifespan (Diehl, Coyle, \& Labouvie-Vief, 1996). 


\subsection{Chapter Two: Conclusion}

Studies One and Two aimed to comprehensively explore the associations between alexithymia, negative affect (depression and anxiety), impulsivity, emotional eating and BMI and develop a novel theoretical model that was inclusive of these factors. In spite of a number of limitations, the two studies offer novel insight into the relationship between alexithymia and emotional eating, as well as BMI. They also highlight important methodological considerations for future research. For the first time, multiple mediating factors (negative affect, negative urgency) were incorporated into one inclusive theoretical model of emotional eating and BMI, and outcomes were tested for robustness through self-replication. Exploratory analysis in a student sample revealed that negative affect (anxiety and depression) and negative urgency mediated the relationship between alexithymia and emotional eating. In comparison, in the more representative sample negative affect (anxiety and depression) was the only significant mediator. In the student sample, difficulty identifying feelings predicted BMI both directly and indirectly via impulsiveness and emotional eating. Testing the applicability of the original model in a more representative general population sample revealed that alexithymia predicted BMI indirectly via negative affect or negative urgency. These findings add strength to the proposal that alexithymia is an important factor to consider when exploring emotional eating and BMI, although its exact influence warrants further investigation. The current findings can also only be applied to self-report emotional eating, and as presented in Chapter One, self-report and behavioural measures of emotional eating are not always consistently related. To overcome this, one way of advancing the models developed in Chapter Two would be to explore actual food intake as a measure of emotional eating. 


\subsection{Chapter Two: Key Findings}

- Alexithymia plays an important role in emotional eating and in turn BMI.

- Alexithymia, emotional eating, negative affect and negative urgency are all significantly correlated constructs.

- Within the student sample, negative affect and negative urgency significantly mediated the relationship between alexithymia and emotional eating, whereas in the more representative sample, it was through negative affect alone.

- Difficulty identifying feelings was directly related to BMI in the student sample.

- Depression significantly mediated the relationship between alexithymia and BMI in the more representative sample, as well as anxiety and negative urgency.

- The development of models requires robust testing and caution should be taken when interpreting models developed solely in student population samples. 


\section{Chapter Three: Development of a novel mood manipulation paradigm}

\subsection{Introduction}

The previous chapter explored the associations between alexithymia and emotional eating, finding that alexithymia was both directly and indirectly related to self-reported emotional eating through different mechanisms; negative affect and negative urgency. To investigate these findings further, Chapters Three and Four explore the associations between alexithymia and emotional eating in a different context; the relationship between alexithymia and food intake during emotional states. One method of achieving this is to manipulate mood followed by a bogus taste test; commonly regarded as an objective measure of emotional eating. However, owing to their relative simplicity (Mills \& D'Mello, 2014), it is essential that robust and effective mood induction procedures are utilised when examining specific behaviours arising in response to emotional and mood states. Therefore, prior to experimentally testing the relationship between alexithymia and food intake (Chapter Four), the aim of the current chapter is to review the literature to determine the variety of mood induction paradigms available, focussing specifically on their effectiveness for eliciting changes in mood and emotional state. It will then conclude by outlining the development of a novel mood induction paradigm, followed by the results of a pilot study examining the effectiveness of a proposed paradigm in inducing changes in mood and feelings of emotional confusion as an analogue to alexithymia. 


\subsection{Literature Review of Mood Induction Procedures}

Mood induction procedures are designed to elicit a specific and transitory emotional state in an artificial and controlled environment (Jallais \& Gilet, 2010). Methods range from film excerpts (direct passage from a film; e.g. Gross \& Levenson, 1995); video clips (amateur video clips; e.g. Kreibig, Samson, \& Gross, 2013; Samson, Kreibig, Soderstrom, Wade, \& Gross, 2016); Velten technique ; autobiographical memories (e.g. Appelhans et al., 2011; Fay \& Finlayson, 2011); music (e.g. Bongers, de Graaff, \& Jansen, 2016; Cardi, Esposito, Clarke, Schifano, \& Treasure, 2015), and stress induction tasks (Haynes, Lee, \& Yeomans, 2003; Royal \& Kurtz, 2010; see Table 13 for a comprehensive overview of available methods within eating behaviour research). For example, film excerpts from "The Champion" and "Green Mile" have successfully induced decreases in mood, whilst excerpts from films such as "When Harry met Sally" and "Mr Bean" have elicited increases in positive affect (e.g. Bongers et al., 2013; Dingemans, Martijn, Jansen, et al., 2009; Dingemans, Martijn, van Furth, et al., 2009). Therefore, even though such methods may not reliably induce specific emotions, they can induce changes in general emotional states (e.g. positive, negative; Izard, 2007; Mills \& D’Mello, 2014).

Within the mood induction literature, the concept of 'mixed emotions' (Larsen \& McGraw, 2014; Samson et al., 2016) is commonly referenced. 'Mixed emotions' can be defined as the co-occurrence of positive and negative affect, or more broadly, as the co-occurrence of two of more emotions at the same time (Larsen \& McGraw, 2014). However, there is some debate in the literature concerning whether valence is an irreducible aspect of the experience of emotion or whether emotional experience, such as positivity and negativity, are separable in experience (see Barrett, 2006; Barrett 
\& Bliss-Moreau, 2009). If the former, it logically follows that people cannot feel happy and sad, or experience fear and anxiety, at the same time. Indeed, it has even been argued that the presence of one emotion destroys the experience of another (Bain, 1859, page 441). However, if the latter, then it follows that people can experience mixed emotions. Although, it could be argued that if/when we experience mixed emotions, we are simply wavering between two or more emotions. Even so, there is an accumulating body of evidence supporting the idea that people can experience mixed emotions (Hunter, Schellenberg, \& Schimmack, 2010; Larsen, McGraw, \& Cacioppo, 2001; Stanley \& Meyer, 2009).

For example, Larsen \& McGraw (2011) conducted a series of studies exploring mixed emotions, finding that participants were more likely to press buttons for happy and sad simultaneously when watching a bittersweet film excerpt than during a control excerpt from "Life is Beautiful". In addition, compared to participants who watched a control film excerpt, participants who watched the bittersweet film excerpt were more likely to describe mixed emotions when responding to open-ended measures of emotion and to list more than one emotion when asked how they were feeling. Interestingly, they also found that this pattern of results persisted even when subjects were told that they were not expected to experience mixed emotions. The finding was also observed among participants who expressed belief in the idea that different emotions cannot be experienced at the same time. Consequently, there appears to be enough evidence to support the idea of mixed emotions, and subsequently, it may also be possible to elicit feelings of emotional confusion and mixed emotional states experimentally via mood induction paradigms.

Consistent with this, Kreibig et al. (2013) found evidence to support the use of film clips to elicit mixed emotions. In this study, 43 women watched film clips that 
elicited amusement, disgust, or mixed emotions (a combination of amusement and disgust) while self-report feelings, respiratory, cardiovascular, electrodermal and facial electromyography measures were assessed. Overall, higher ratings of mixed emotions were reported following the mixed emotion video clips compared to amusing or disgusting only video clips. The mixed emotion condition also elicited a unique psychophysiological response and similar intensities of amusing and disgusted feelings were reported following the mixed emotions condition (combined amusement and disgust), supporting the idea of a mixed emotional state. However, it should be noted that Kreibig et al. (2013) only used females in their study and caution needs to be exercised when generalising their results to a broader population. In addition, Samson and colleagues (2016) developed a library of video clips to induce changes in positive (amusement), negative (repulsion and fear) and mixed emotions (a blend of amusement and repulsion/fear), finding that even though mixed emotion video clips elicited moderate levels of positive and negative emotions, single emotion video clips elicited higher intensities of their target emotions. Overall, the elicitation of less intense mixed emotions compared to single emotions poses a possible limitation of the use of video clips in mood induction paradigms.

More generally, studies utilising film excerpts to elicit and induce changes in mood and emotional states have also received wider criticism (e.g. Gabert-Quillen, Bartolini, Abravanel, \& Sanislow, 2015; Hewig et al., 2005; Samson et al., 2016). First, a review focussing on a number of studies using film excerpts found that sex, race, ethnicity and familiarity all had an impact on the intensity of emotions experienced by participants (Gabert-Quillen et al., 2015) and differences have also been found across age groups (Samson et al., 2016). This highlights that film excerpts may exert a different impact on one person compared to another. Second, even though 
some studies have successfully used film excerpts to elicit target emotions, Hewig and colleagues (2005) noted that sitting and watching a film clip (i.e. a passive observation) differed from actively engaging with the emotion. For instance, a film excerpt arguably lacks ecological validity as it does not capture the richness and complexity of real-life situations in which emotions occur. As explained by Evers et al. (2009), watching a film excerpt means that the observer is detached from the situation, rather than being the actor in the emotional situation. They also noted that film excerpts routinely used within research tend to focus heavily on western culture, and consequently, targeted emotions are generated from this. Third, film excerpts to induce emotional confusion are not readily available, and when they have been utilised, they tend to have a narrow focus (i.e. amusement and repulsion/fear clips by Samson et al., 2016). As a result, such clips can fail to capture the wide array of possible mixed emotions associated with the human experience. Finally, even though some authors have used film excerpts to induce mixed feelings (Larsen \& McGraw, 2011; Schaefer, Nils, Sanchez, \& Philippot, 2010), no known studies have used films to induce feelings of emotional confusion specifically. Therefore, given that film excerpts have produced mixed findings, that they have been found to impact differently on people depending on individual differences, and are heavily influenced by culture, film excerpts and video clips were not deemed a viable methodological option.

In contrast, Velten procedures, which involve presenting participants with self-referent statements and asking them to feel the mood described, may be an alternative method (Velten, 1968). However, it can be easier to detect the aims of the research when using such procedures (Polivy \& Doyle, 1986, as cited in Västfjäll, 2001), and consequently, evidence for their effectiveness is very mixed (Kenealy, 1986). Similar criticism has also been applied to mood induction procedures using 
music. Specifically, whilst music based mood induction paradigms can successfully elicit changes in mood, they are also fairly susceptible to demand characteristics, including participants more readily perceiving the research hypothesis (Västfjäll, 2001). Finally, stress tasks, most commonly the Trier Social Stress Test (TSST; Kirschbaum, Pirke, \& Hellhammer, 1993), have also been found to effectively elicit changes in levels of stress (Hellhammer, Kirschbaum, Kudielka, \& Hellhammer, 2007). Nevertheless, inducing heightened levels of stress is too broad and does not effectively address the aims of the current study.

In contrast to film excerpts, video clips, Velten procedures, stress induction tasks and the use of music, a method that seems to hold more promise is autobiographical recall. Using autobiographical recall in mood induction paradigms is thought to result in a more intense emotional experience (Lane, Reiman, Ahern, Schwartz, \& Davidson, 1997) and can be more ecologically valid (Berrios, Totterdell, $\&$ Kellett, 2015a). Furthermore, such internal procedures (e.g. autobiographical recall) are considered to be at least equally effective at inducing target emotions as external procedures (e.g. film excerpts; Salas, Radovic, \& Turnbull, 2012). For example, autobiographical recall has been shown to elicit significant changes to both valence and arousal, especially for happiness and sadness, and has been found to be more effective than music and guided imagery methods combined (Jallais \& Gilet, 2010). Even so, the literature concerning the use of autobiographical recall to induce mixed emotions or emotional confusion is limited. However, one study has used the technique to explore recalling the experience of conflicting goals as a precursor of mixed emotions (Berrios, Totterdell, \& Kellett, 2015b). Berrios et al. (2015b) asked participants to recall either a recent conflicting or facilitating goal event as vividly as possible. Conflicting goals were defined as experiencing a discrepancy between 
desired outcome and the progress towards the outcome (Carver \& Scheier, 1990). They found that participants reported higher mixed emotions scores when recalling an event in which goals were conflicted rather than facilitated. This supports the use of autobiographical recall in the induction of mixed emotions, and potentially, could be a viable method to induce emotionally confusing mood states also.

Fay and Finlayson (2011) suggested that autobiographical recall encourages a more naturalistic mood induction as the technique involved is similar to diary-writing; reducing participant feelings of embarrassment and self-consciousness. Autobiographical recall is also a relatively easy and time-efficient method of inducing mood and emotional states in laboratory settings (Mills \& D’Mello, 2014). In addition, autobiographical recall also overcomes several of the limitations associated with external procedures discussed earlier in this chapter (i.e. passivity; individual differences). Finally, owing to the adaptability of autobiographical recall methods, it is fairly easy to tailor task instructions to target specific and mixed emotional states (e.g. emotional confusion). For example, it is easy to draw reference to particular features of alexithymia and specific items of the TAS-20 alexithymia scale (i.e. a time where it has been difficult to identify which emotions are being experienced or to describe them to someone else) in task instructions to allow the induction of emotional confusion as an analogue of alexithymia.

\subsection{Study Three: Aims}

The overarching aim of Study Three (pilot study) was to develop and test a novel mood manipulation procedure that could effectively induce positive, negative and neutral (as a control) emotional states, as well as induce emotionally confusing 
feelings as an analogue of alexithymia. A key objective was to examine the effect of autobiographical recall of positive, negative, neutral (control) or emotionally confusing events, on ratings of general mood state as captured by total and subscale scores of the Profile of Mood States (POMS; clear-headed, composure, elatedness, energetic, agreeable, confident) as well as the Subjective measure of Mixed Emotion (SME).

For the control condition, no significant changes in POMS subscale scores were expected from Time 1 and 2 ( $T_{1}$ and $T_{2}$; pre- and post-mood manipulation). For participants in the positive condition, elatedness scores were predicted to increase following manipulation of mood, with the opposite predicted to occur in the negative condition. For the emotionally confusing condition, a decrease in participant's selfreported level of clear-headedness was predicted, as well as an increase in 'mixed emotions' (SME) ratings from $T_{1}$ to $T_{2}$.

Using the Linguistic Inquiry and Word Count (LIWC; Pennebaker, Boyd, Jordan, \& Blackburn, 2015) programme, the pilot study also analysed the emotional content and written expression of affect in the autobiographical scripts written to describe positive, negative, neutral (control) and emotionally confusing events. The resultant themes were examined as an additional way to assess the validity of the novel mood induction paradigm, and specifically, the effectiveness of using autobiographical recall to induce feelings of emotional confusion. 


\subsection{Study Three: Method}

\subsubsection{Participants}

To be eligible to take part, participants had to be between 18-65 years of age and report no history of mood disorders. Seventy-six participants completed the study, although data for one participant was subsequently removed as their self-reported BAI and BDI scores fell in the 'severe' range. Of the final 75 participants, $58(77.3 \%)$ were female and mean age was 21.89 years of age $(S D=5.55$ years, range $=18.83-59.59$ years). Two participants incorrectly inputted their date of birth, and consequently, these data points were marked as missing values. The majority of the sample were single $(n=70 ; 93.3 \%)$, with the remaining either married $(n=1,1.3 \%)$, co-habiting $(n$ $=1,1.3 \%)$ or separated/divorced $(n=3,4 \%)$. Ethnicity was recorded as $69(92.0 \%)$ White British, two (2.7\%) Asian, one (1.3\%) Chinese, one (1.3\%) Mixed, one (1.3\%) reported other and one (1.3\%) preferred not to say. At the time of the study, $64(85.3 \%)$ participants were in full/part-time education, eight (10.6\%) were in full/part-time employment, two $(2.7 \%)$ were unemployed, and one (1.3\%) volunteered. To reduce bias and potential confounding variables (see Schulz \& Grimes, 2002), participants were systematically assigned to one of four groups: negative $(n=19)$; positive $(n=$ $19)$; emotional confusion $(n=19)$, or control $(n=18)$. In order to keep the size of groups similar, random allocation in blocks was conducted. A random sequence of groups (1-4) was generated in blocks of four and repeated.

An a priori power analysis ( $\mathrm{G}^{*}$ Power; Faul et al., 2007) indicated that a minimum sample size of 104 (26 per group) would be required (effect size $=.25-$ 
medium; power $=.8$ ). However, as this was a pilot study, only 75 participants were recruited for initial examination of the mood induction paradigm.

\subsubsection{Measures}

\subsubsection{Demographics}

The following information was sought from all participants: date of birth, sex, marital status, employment status, ethnicity, and number of years spent in education.

\subsubsection{Profile of Mood States}

(POMS; Lorr \& McNair, 1982; McNair, Lorr, \& Droppleman, 1971, 1992). The POMS is a 72-item measure used to assess respondent's current mood state. Participants are asked to select the answer that best describes "how they are feeling today, at this point", on a 4-point scale (0 "much unlike this" to 3 "much like this"). In addition to an overall mood state score, the POMS generates six bipolar subscales with six positive and six negative mood markers (Lorr, McNair, \& Fisher, 1982): composed-anxious (e.g. "untroubled; nervous"); agreeable-hostile (e.g. "kindly; angry"); elated-depressed (e.g. "cheerful; dejected"), confident-unsure (e.g. "strong; timid"); energetic-tired (e.g. "lively; fatigued"), and clearheaded-confused (e.g. "attentive; mixed-up"). Subscales are calculated by subtracting the sum of negative mood markers from the sum of positive mood markers for each subscale. The POMS was completed pre- and post-manipulation ( $T_{1}$ and $T_{2}$, respectively) and had good internal consistency, $T_{1}: \alpha=.85$ and $T_{2}: \alpha=.87$. Although some studies have argued that the collection of pre-manipulation mood ratings prime participants to expect changes in mood, Mills and D'Mello (2014) found that the collection of pre- 
manipulation mood ratings did not impact upon results. Therefore, POMS data was collected pre-manipulation in line with previous protocols.

\subsubsection{Subjective measure of Mixed Emotion}

(SME; Berrios, Totterdell, \& Kellett, 2013; Berrios et al., 2015b; Berrios, Totterdell, \& Kellett, 2017). The SME is a four-item subjective scale measuring mixed emotions. Participants are asked to rate the extent to which they have been experiencing mixed emotions, (e.g. "I'm feeling contrasting emotions; I'm feeling a mixture of emotions") on a five-point Likert-type scale from 1 ("not at all") to 5 ("very much"). The sum of all responses is averaged to give a single overall score of mixed emotions. High levels of internal consistency were found at both $T_{1}: \alpha=.91$ and $T_{2}: \alpha$ $=.94$, which is in line with previous research in similar demographic samples (Berrios et al., 2015b, 2017; Berrios, Totterdell, \& Kellett, 2018).

\subsubsection{Toronto Alexithymia Scale}

(TAS-20; Bagby, Parker, et al., 1994; Bagby, Taylor, et al., 1994). The TAS20 is fully described in Chapter Two (section 2.3.2.3). In the current study, high levels of internal consistency were found for total $(\alpha=.83)$, DIF $(\alpha=.84)$ and $\operatorname{DDF}(\alpha=.85)$ subscales. The EOT subscale was found to have low internal consistency $(\alpha=.31$ ), although a relatively low internal reliability alpha is not uncommon for this subscale (for a review see Kooiman, Spinhoven, \& Trijsburg, 2002).

\subsubsection{Beck Depression Inventory-II}

(BDI; Beck et al., 1961; Beck et al., 1996; Beck, Steer, et al., 1988). The BDI is fully outlined in Chapter Two (section 2.3.2.5.) and was used in the current study to control for levels of depression. A high level of internal consistency was found ( $\alpha=$ .87). 


\subsubsection{Beck Anxiety Inventory}

(BAI; Beck, Epstein, et al., 1988). The BAI is fully outlined in Chapter Two (section 2.3.2.6) and was used here to control for levels of anxiety. A high level of internal consistency was found $(\alpha=.89)$.

\subsubsection{Memory Characteristics Questionnaire}

(MCQ; Johnson, Foley, Suengas, \& Raye, 1988). The MCQ assesses a range of characteristics associated with memories, such as sensory detail, feelings and vividness. Participants respond by selecting the answer that best captures how vivid/detailed (as examples) their recalled memory is on a seven-point scale from 1-7, with anchors such as "vague" (1) to "clear/distinct" (7) and "not intense" (1) to "very intense" (7). Scores on each item are then summed to derive subscale scores for (a) clarity (defined as how clear the memory is, e.g. "My memory for this event is sketchy/very detailed"); (b) sensory (defined as how the memory is remembered across the senses, e.g. "My memory for this event involves smell/taste" etc.); (c) contextual factors (defined as the recall of the spatial arrangement of the memory, e.g. "My memory for the location where the event took place is vague/clear"), and (d) time (defined as when the event took place, e.g. "My memory for the season/day when the event took place is vague/clear"; McGinnis \& Roberts, 1996; Schaefer \& Philippot, 2005; Suengas \& Johnson, 1988). Moderate to high levels of internal consistency were found for clarity $(\alpha=.86)$, sensory $(\alpha=.71)$ and time $(\alpha=.74)$ subscales across groups but low internal consistency was found for the context subscale $(\alpha=.49)$. However, Schaefer and Philippot (2005) found similar Cronbach's alphas in their study when exploring the four subscales following positive, negative and neutral memory recollection. Of particular interest in the current study was the clarity subscale and items that assessed the overall tone, valence of feelings at the time, and intensity of the 
feelings at the time and at present. The MCQ was also used in the current study to help add authenticity to the cover story.

\subsubsection{Stimuli}

Participants were presented with different instructions depending on the condition. Participants assigned to the positive and negative groups were asked to recall a time in which they had felt "happy" or "sad" respectively, with participants in the control group asked to recall their usual journey to work or university. The task instructions for the emotionally confusing group used items from the TAS-20 alexithymia scale to tap into the emotions and sensations associated with alexithymia and the experience of emotional confusion. For example, "Being in touch with our emotions is essential" (item 10), and "This can include not being able to find the right words for how you are feeling" (item 2). The utilisation of TAS-20 items aimed to encourage participants to focus on a time they had experienced difficulty identifying and/or describing their emotions in a particular situation or in response to a specific event. It also helped to ensure emotional confusion was being induced as an analogue of alexithymia. The full instructions given to be participants can be found in Appendix Q.

\subsubsection{Procedure}

Ethical approval was obtained from the Department of Psychology Ethics Committee, College of Human and Health Sciences, Swansea University. 
Participants were recruited through the Department of Psychology's participant pool in exchange for course credit, or through adverts/flyers in which participants received payment (£3.50). The study was advertised as an investigation into "Memory Recall" and was described to participants as an exploration of how well individuals can recall personal experiences and events, including how well specific details are remembered. All participants provided standard demographic information and completed Time $1\left(T_{1}\right)$ POMS and SME, as well as the TAS-20, BDI and BAI via a digitalised questionnaire using Survey Monkey (Palo Alto, California, USA). Once completed, participants notified the experimenter and were presented with the instructions for either the positive, negative, emotionally confusing or control group. The experimenter read the study protocol instructions aloud to the participants and left a printed copy for reference (See Appendix Q). As an example, participants in the positive group were presented with the following instructions:

"During the next 7 minutes, I would like you to think about an event in which you have personally felt happy. I would like you to recall and write about such an event as vividly and in as much detail as possible.

I would like you to picture the event happening again to you and immerse yourself in the thoughts you had at the time. Also, think about what led up to that particular situation and how you felt about it and the outcome. Anything you write down will remain anonymous and confidential, so feel free to write what you like to help you fully re-experience how you felt at the time."

Participants were given seven minutes to recall and write about their chosen memory before the experimenter re-entered the room. Participants were then instructed to complete Time $2\left(T_{2}\right)$ POMS, as well as the SME and MCQ. The study lasted 
approximately 30 minutes and participants were thanked for their time and debriefed before receiving payment or course credit.

\subsubsection{Statistical Analysis}

\subsubsection{Quantitative Statistics}

A missing values analysis revealed nine missing POMS values across $T_{l}$ and $T_{2}$ (pre- and post-mood manipulation). Missing values were replaced by averaging the positive or negative items within the same subscale and using the calculated value. All analysis was performed using SPSS 22.0.

A 4 × 2 mixed ANOVA was conducted to examine whether POMS (total and subscale) and SME scores significantly differed pre- $\left(T_{1}\right)$ and post-manipulation $\left(T_{2}\right)$. All assumptions to conduct these analyses were met. One-way ANOVA's were also conducted to explore MCQ (memory characteristics) differences across groups. Specifically, the clarity subscale of the MCQ as well as individual items that assessed the overall tone, valence of feelings at the time, and intensity of the feelings at the time and at present were analysed. Post-hoc analyses (Bonferroni) were conducted where appropriate. When homogeneity of variance was violated, Brown-Forsythe $F$-ratio was reported and Games-Howell post-hoc analyses were conducted.

\subsubsection{Qualitative Statistics}

All handwritten memory scripts from the autobiographical recall tasks were transcribed into computer text files. Any words that were deemed illegible were shown to other members of the research team (supervisors) and a consensus derived. Summative content analysis, also referred to as manifest content analysis (Potter \& Levine-Donnerstein, 1999), was then conducted to explore the range of topics 
participants reported when asked to think about a positive, negative or emotionally confusing situation. Summative analysis identifies content in text to facilitate understanding of the contextual use of the content (Hsieh \& Shannon, 2005). There is no attempt to infer meaning from the analysis and purely quantitative results are generated. One participant listed the particular emotions experienced during their chosen event but did not provide details about the event itself. A further participant failed to record their memories in writing as instructed. Consequently, data from these two participants was excluded from the qualitative analysis.

\subsection{Linguistic Inquiry and Word Count}

Linguistic inquiry and word count (LIWC; Pennebaker, Chung, Ireland, Gonzales, \& Booth, 2007; Pennebaker, Francis, \& Booth, 2001; Pennebaker et al., 2015). LIWC (2015 version) is a computer-based programme that counts the emotional expression of words over three hierarchies. The first hierarchy counts the total number of emotion or affect words present (1393 words; Kahn, Tobin, Massey, \& Anderson, 2007). In the second hierarchy, emotional words are split into positive and negative words. Six hundred and twenty positively valenced (e.g. "love; sweet") and 744 negatively valenced words or feelings (e.g. "nasty; ugly") are contained within the positive and negative emotion categories, respectively. The final hierarchy breaks the negative emotion category down further, including anxiety/fear (116 words; e.g. “worried; fearful"), anger (230 words; e.g. "hate; annoyed") and sadness/depression (136 words; e.g. "grief; sad"). Many words are counted in multiple categories (e.g. “cried' falls into overall affect, negative emotions and sadness/depression), and each category consists of either whole words or word stems (e.g. "cry; cries; cried"). Word count results are presented as a percentage of the total number of words in the text. 
A series of one-way ANOVA's compared the word counts at each hierarchy across the four groups, with simple planned contrasts used to further investigate the main effects.

\subsection{Study Three: Results}

\subsubsection{Descriptive Statistics}

TAS-20, BDI and BAI scores were in line with the distribution found in similar samples (see Chapter Two, section 2.4.1.; Table 9).

\subsubsection{Quantitative Results}

\subsubsection{Manipulation Check: Comparison of POMS and SME scores pre- and post-mood manipulation $\left(T_{1}\right.$ and $\left.T_{2}\right)$}

To determine the effectiveness of the mood manipulation, SME and POMS total and subscale scores were compared across groups and from $T_{1}$ and $T_{2}$.

Total POMS Score. There was no significant main effect of time, $F(1,71)=$ $.001, p=.980$, or group, $F(3,71)=2.66, p=.055$. However, a significant time $\mathrm{x}$ group interaction was found, $\left.F(3,71)=3.05, p=.034, \eta^{2}=.114\right)$. Post-hoc analysis revealed a significant increase in overall mood from $T_{1}$ to $T_{2}$ in the positive group $(p=.028$, see Table 10 and Figure 9). Participants in the negative group reported significantly lower overall mood than participants in the control $(p=.027)$ and positive groups $(p=.039)$ at $T_{2}$, see Table 10 and Figure 9. 
Table 9. Total and group mean, standard deviation and range for alexithymia, depression and anxiety for Stuc

\begin{tabular}{lccccccc}
\hline & \multicolumn{2}{c}{ Total } & \multicolumn{2}{c}{ Positive } & \multicolumn{2}{c}{ Negative } & Emotional Co \\
\cline { 2 - 7 } & Mean $(S D)$ & Range & Mean $(S D)$ & Range & Mean $(S D)$ & Range & Mean $(S D)$ \\
\hline DIF & $12.79(4.60)$ & $6-26$ & $12.79(3.36)$ & $7-19$ & $14.32(6.05)$ & $7-26$ & $13.26(4.51)$ \\
DDF & $12.75(5.10)$ & $5-25$ & $13.53(5.05)$ & $5-22$ & $11.68(5.54)$ & $5-23$ & $14.58(4.9)$ \\
EOT & $18.89(3.47)$ & $11-31$ & $20.26(3.94)$ & $15-31$ & $17.53(2.97)$ & $12-23$ & $19.00(3.02)$ \\
TAS-20 & $44.43(10.11)$ & $26-68$ & $46.58(8.79)$ & $30-60$ & $43.53(13.18)$ & $26-68$ & $46.84(8.97)$ \\
BDI & $10.08(7.25)$ & $0-31$ & $10.58(7.96)$ & $0-23$ & $11.42(7.5)$ & $0-29$ & $10.32(6.97)$ \\
BAI & $10.80(8.60)$ & $0-36$ & $11.89(9.02)$ & $0-28$ & $13.47(9.76)$ & $0-30$ & $10.32(7.64)$ \\
\hline BAI = Beck anxiety inventory, BDI = Beck depression inventory, DDF = difficulty describing feelings, DIF = & EOT = externally oriented thinking, TAS-20= Toronto alexithymia scale & &
\end{tabular}




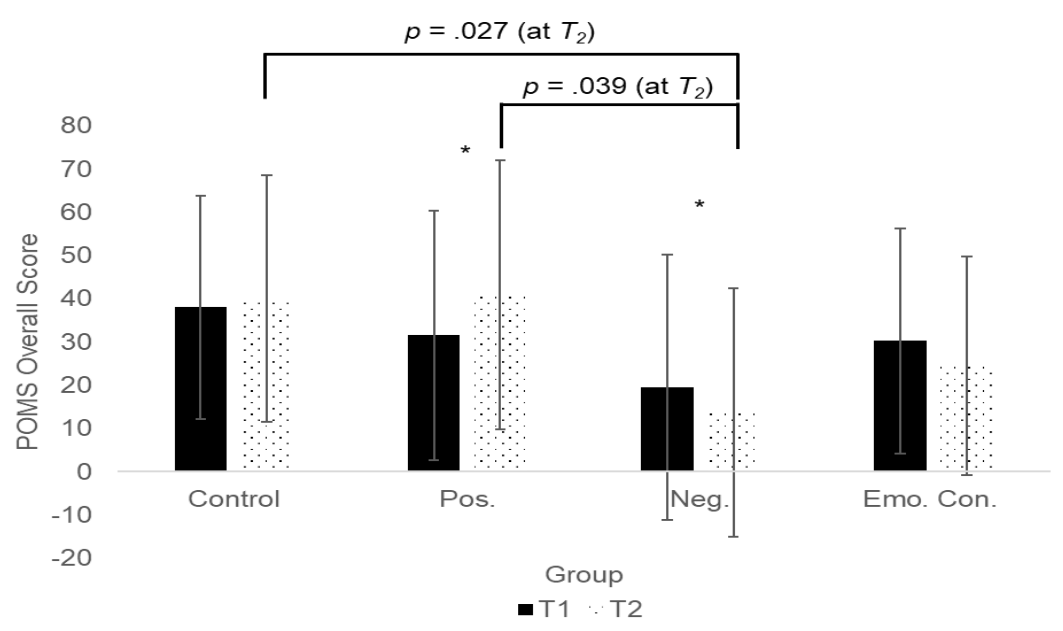

Figure 9. Overall POMS scores for control, positive, negative and emotional confusing group. Error bars represent SD. (* $=$ significant difference between $T_{1}$ and $T_{2}$ within group).

Clear-headedness. There was no significant main effect of time, $F(1,71)=.28$, $p=.601$, or group, $F(3,71)=1.72, p=.171$. In addition, there was no significant time $\mathrm{x}$ group interaction, $F(3,71)=2.41, p=.074$. However, post-hoc analysis found a significant decrease in clear-headed scores from $T_{1}$ and $T_{2}(p=.030$, see Table 10) in the emotionally confusing group.

Elatedness. There was no significant main effect of time, $F(1,71)=3.27, p=$ .075 , but a significant main effect of group was found, $F(3,71)=4.69, p=.005, \eta^{2}=$ .165. The time $\mathrm{x}$ group interaction was also significant, $F(3,71)=5.14, p=.003, \eta^{2}=$ .18). Post-hoc analysis revealed a significant increase in elatedness scores from $T_{1}$ to $T_{2}$ in the positive group $(p=.03)$ and a significant decrease in the negative group $(p=$ .003 , see Table 10 and Figure 10). At $T_{2}$, elatedness scores were significantly higher in the positive than negative $(p<.001)$ and emotional confusing groups $(p=.005)$, and 
significantly higher in the control versus negative group $(p=.019$, see Table 10 and Figure 10).

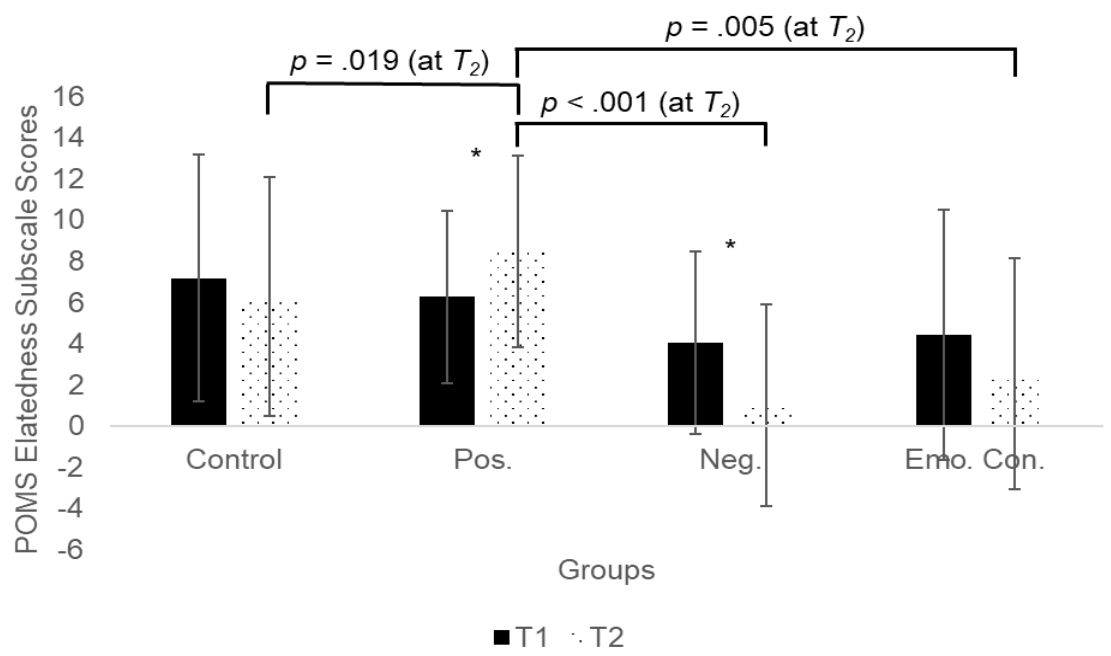

Figure 10. POMS elatedness subscale scores for control, positive, negative and emotional confusing groups. Error bars represent SD. $\left(^{*}=\right.$ significant difference between $T_{1}$ and $T_{2}$ within group).

Composed. There was no significant main effect of time, $F(1,71)=.98, p=$ .326 , and no significant time $\mathrm{x}$ group interaction, $F(3,71)=1.25, p=.298$. However, the main effect of group was significant, $F(3,71)=2.96, p=.038, \eta^{2}=.111$, with POMS composure scores significantly decreasing from $T_{1}$ to $T_{2}$ in the emotionally confusing group $(p=.041$, see Table 10).

Energetic. A significant main effect of time was found for the energetic subscale, $\left.F(1,71)=9.31, p=.003, \eta^{2}=.116\right)$, but the main effect of group was not significant, $F(3,71)=1.64, p=.188$. The time $x$ group interaction was also not significant, $F(3,71)=1.81, p=.154$. Post-hoc analysis revealed a significant increase in energetic scores from $T_{1}$ to $T_{2}$ in the positive group ( $p=.002$, see Table 10$)$. 
Confident. There was no significant main effect of time, $F(1,71)=1.53, p=$ .22 , or group, $F(3,71)=1.99, p=.123$. The interaction between time $\mathrm{x}$ group was also not significant, $F(3,71)=5.1, p=.623$, and post-hoc analysis revealed no significant differences across groups.

Agreeableness. There was no significant main effect of time, $F(1,71)=.89, p$ $=.35$, or group, $F(3,71)=.8, p=.498$. A significant time $\mathrm{x}$ group interaction was found, $\left.F(3,71)=3.88, p=.0013, \eta^{2}=.141\right)$, with participants in the negative group reporting feeling significantly less agreeable following the mood manipulation $(p=$ .009 , see Table 10).

SME. There was no significant main effect of time, $F(1,71)=.07, p=.797$, but the main effect of group was significant, $F(3,71)=2.87, p=.042, \eta^{2}=.108$. A significant time $\mathrm{x}$ group interaction was also found, $\left.F(3,71)=3.99, p=.011, \eta^{2}=.144\right)$. Participants in the emotionally confusing group reported significantly greater mixed emotions at $T_{2}$ compared to $T_{1}(p=.003$, see Table 10 and Figure 11). Participants in the emotionally confusing group also scored significantly higher on the SME compared to those in the control group ( $p=.026$, at $T_{2}$, see Table 10 and Figure 11).

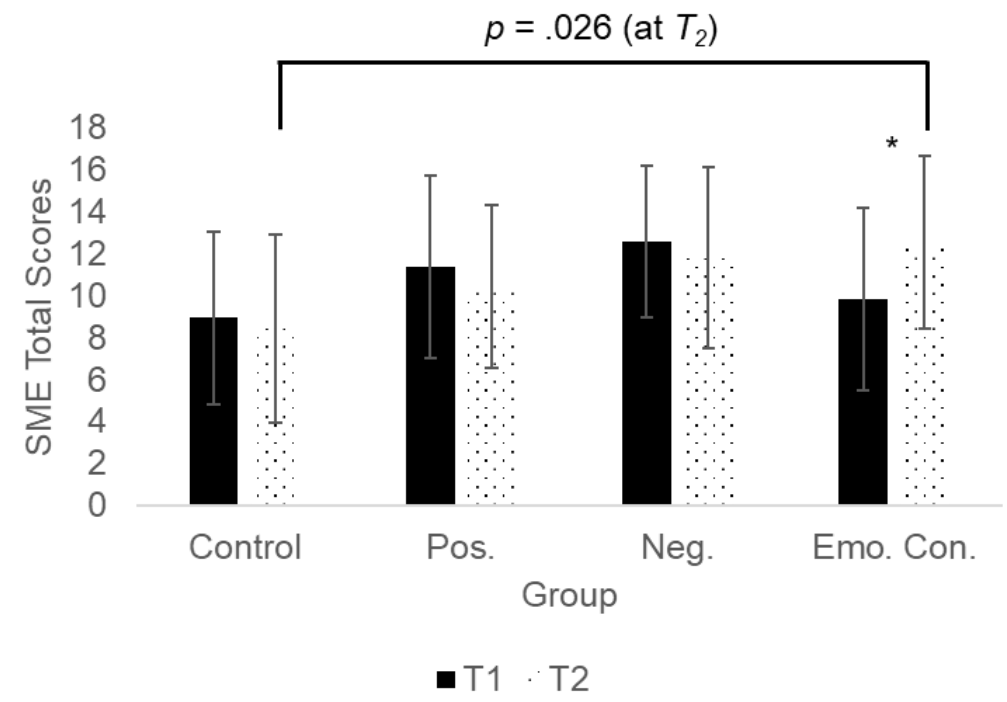

Figure 11. SME scores for control, positive, negative and emotional confusing groups. Error bars represent SD. ( ${ }^{*}=$ significant difference between $T_{1}$ and $T_{2}$ within group). 
Table 10. Baseline $\left(T_{1}\right)$ and post-manipulation $\left(T_{2}\right)$ means and standard deviations for the POMS subscales,

\begin{tabular}{|c|c|c|c|c|c|c|c|c|c|c|c|c|}
\hline & \multicolumn{2}{|c|}{ Elatedness } & \multicolumn{2}{|c|}{$\begin{array}{c}\text { Clear- } \\
\text { headedness }\end{array}$} & \multicolumn{2}{|c|}{ Composed } & \multicolumn{2}{|c|}{ Energetic } & \multicolumn{2}{|c|}{ Confident } & \multicolumn{2}{|c|}{ Agreeableness } \\
\hline & $T_{1}$ & $T_{2}$ & $T_{1}$ & $T_{2}$ & $T_{1}$ & $T_{2}$ & $T_{1}$ & $T_{2}$ & $T_{1}$ & $T_{2}$ & $T_{1}$ & $T_{2}$ \\
\hline \multirow[t]{2}{*}{ Con. } & 7.17 & 6.28 & 7.28 & 7.56 & 8.83 & 8.56 & 2.22 & 3.44 & 3.28 & 4.39 & 9.28 & 9.83 \\
\hline & $(5.99)$ & (5.82) & $(4.38)$ & $(5.22)$ & $(5.71)$ & $(6.62)$ & $(5.61)$ & $(6.35)$ & (4.57) & $(5.61)$ & $(4.36)$ & $(4.51)$ \\
\hline \multirow[t]{2}{*}{ Pos. } & 6.26 & 8.47 & 6.00 & 7.21 & 7.16 & 7.74 & -.37 & 2.42 & 3.32 & 4.63 & 9.16 & 10.37 \\
\hline & $(4.21)$ & (4.66) & (7.13) & $(6.36)$ & $(5.87)$ & $(4.05)$ & (7.12) & $(8.24)$ & $(5.53)$ & $(6.73)$ & $(4.27)$ & $(4.47)$ \\
\hline \multirow[t]{2}{*}{ Neg. } & 4.05 & 1.00 & 4.00 & 3.47 & 3.63 & 3.42 & -1.47 & -1.58 & .53 & .68 & 8.79 & 6.74 \\
\hline & $(4.44)$ & (4.88) & $(5.76)$ & $(5.85)$ & $(7.25)$ & $(6.57)$ & (6.99) & $(5.74)$ & $(6.40)$ & $(5.53)$ & (4.09) & $(5.00)$ \\
\hline Emo. & 4.42 & 2.53 & 7.16 & 5.32 & 7.05 & 4.89 & 1.00 & 2.53 & 1.42 & 1.21 & 9.16 & 8.00 \\
\hline Con. & (6.06) & (5.59) & (4.62) & (4.27) & (5.35) & (6.15) & $(6.65)$ & $(5.07)$ & $(5.36)$ & $(5.48)$ & $(5.10)$ & (6.13) \\
\hline
\end{tabular}

Emo. Con. = emotionally confusing group, Neg. = Negative group, Cont. = Control group, POMS = Profile 0 group, $\mathrm{SME}=$ subjective measure of mixed emotion. Bold indicates significant differences between $T_{1}$ and $T$ 


\subsubsection{Characteristics of the memory}

A one-way ANOVA found no significant difference across groups for the overall 'clarity' of participants recalled memories, $F(3,71)=.27, p=.849$. A significant difference was found for the overall 'tone' of the memory recalled, and as the assumption of homogeneity of variance was violated, Brown-Forsythe $F$-ratio was reported, $F(3,51.19)=50.34, p<.001$. Post-hoc pairwise comparisons (GamesHowell) revealed that recalled memories in the positive group were significantly more positive in tone $(M=6.5, S D=.71)$ compared to those in the control $(M=5.17, S D=$ $1.43, p=.008)$, negative $(M=1.52, S D=1.07, p<.001)$ and emotionally confusing groups $(M=3.05, S D=1.87, p<.001)$. Likewise, recalled memories in the negative group were significantly more negative in tone compared to those in the control $(p<$ $.001)$ and emotional confusing groups $(p=.022)$. Recalled memories in the emotionally confusing group were also significantly more negative than those in the control group $(p=.002)$.

There was a significant overall difference for "feelings at the time", $F(3,70)=$ $42.35, p<.001$. Specifically, 'feelings at the time" were significantly more positive in the positive $(M=6.22, S D=1.4)$ than control $(M=4.83, S D=1.65, p=.037)$, negative $(M=1.32, S D=.946, p<.001)$ and emotionally confusing groups $(M=2.42, S D=$ $1.77, p<.001)$. Memories recalled in the negative and emotionally confusing groups were also significantly more negative than those recalled in the control group $(p<$ $.001)$.

There was also a significant difference in the "intensity of feelings at the time", $F(3,70)=24.43, p<.001$. Post-hoc analysis revealed that participants in the control group $(M=2.67, S D=1.72)$ reported significantly less intense feelings compared to those in the positive $(M=5.39, S D=1.58, p<.001)$, negative $(M=6.26, S D=.99, p$ 
$<.001)$ and emotionally confusing groups $(M=5.63, S D=1.12, p<.001)$. Finally, there was a significant difference in intensity of feelings at the time of recall, $F(3,70)$ $=10.86, \mathrm{p}<.001)$. Post-hoc analysis revealed that participants in the control group $(M$ $=2.39, S D=1.24)$ reported significantly less intense feelings compared to participants in the positive $(M=5.22, S D=1.58, p<.001)$, negative $(M=4.11, S D=1.37, p=$ $.006)$ and emotionally confusing groups $(M=4.21, S D=1.87, p=.003)$. No other significant differences were found.

\subsubsection{Qualitative Findings}

\subsubsection{Summative content analysis}

As participants could select any memory from their lives that best reflected the emotion they had been asked to recall, a variety of events were chosen. The control group were asked to write about their usual journey to work or university, and these were all classed as "Journey". Four further themes were classified: death/illness; relationships; achievement/failure, and events/milestones (e.g. birthdays, going to university). Categories were then further broken down into subthemes of either positive or negative themes. Table 11 shows the breakdown of theme and the number

of instances that each type of memory occurred in each theme. All autobiographical recalls were housed in a single subtheme. Participants in the positive group reported previous successes and achievements or recalled positive events, such as birthdays and holidays. In comparison, participants in the negative group focused more on death and illness of loved ones and the accompanying sadness. The negative and emotionally confusing group also differed, with participants in the latter group more frequently recalling the difficulties experienced in relationships (i.e. relationship breakdown and 
dissolution). Five participants in the emotionally confusing group also reported the death or illness of a friend or relative.

\subsubsection{Word Count}

The number of affect words used in participant autobiographical scripts differed significantly across groups, $F(3,70)=8.47, p<.001, \eta^{2}=.256$. Planned simple contrasts revealed that participants in the positive $(p=.002)$, negative $(p<.001)$ and emotional confusing $(p<.001)$ groups used significantly more emotional words than those in the control group (see Table 12). There was no significant difference in the number of overall affect words used across the positive, negative and emotionally confusing groups.

In addition, the number of positive affect words used significantly differed across groups, $F(3,70)=11.21, p<.001, \eta^{2}=.324$. As expected, significantly more positive affect words were used in the positive compared to control $(p<.001)$, negative $(p=.013)$, and emotionally confusing groups $(p=.010$, see Table 12$)$. The assumption of homogeneity of variance was violated for the negative affect words and therefore, the Brown-Forsythe $F$-ratio was used. A significant main effect of group was found, $F(2,13.43)=13.43, p<.001, \eta^{2}=.357$, with post-hoc pairwise comparisons (GamesHowell) revealing that significantly more negative affect words were used in the negative compared to the control $(p=.002)$ and positive $(p=.009$, see Table 12$)$ groups. A significantly greater number of negative affect words were also used in the emotionally confusing compared to the control $(p<.001)$ and positive $(p<.001)$ groups, see Table 12. There was no significant difference in the number of negative affect words used across the negative and emotionally confusing groups. 
Table 11. Themes of autobiographical memories recalled in Study Three.

\begin{tabular}{|c|c|c|c|c|}
\hline \multirow[b]{2}{*}{ Themes } & \multicolumn{4}{|c|}{ Group } \\
\hline & Control & Positive & Negative & $\begin{array}{c}\text { Emotionally } \\
\text { Confusing }\end{array}$ \\
\hline Journey & 18 & & & \\
\hline \multicolumn{5}{|l|}{ Death/IIlness } \\
\hline \multicolumn{5}{|l|}{ Negative } \\
\hline Death/illness of a & & & 5 & 3 \\
\hline relative & & & 4 & 2 \\
\hline \multirow{2}{*}{\multicolumn{5}{|c|}{$\begin{array}{c}\text { Death/illness of a } \\
\text { friend }\end{array}$}} \\
\hline & & & & \\
\hline \multicolumn{5}{|l|}{ Relationships } \\
\hline \multicolumn{5}{|l|}{ Positive } \\
\hline Start of a relationship & & 1 & & \\
\hline Making friends & & 1 & & \\
\hline Meeting extended & & 1 & & \\
\hline family & & 1 & & \\
\hline \multicolumn{5}{|l|}{ Engagement } \\
\hline Negative & & & 1 & \\
\hline Cheated on & & & 1 & \\
\hline Bullied & & & & 2 \\
\hline Argument with friends & & & & 1 \\
\hline Relationship & & & & 1 \\
\hline difficulties & & & & 1 \\
\hline Meeting extended & & & 4 & 4 \\
\hline family & & & & 2 \\
\hline \multicolumn{5}{|l|}{ Missing partner } \\
\hline \multicolumn{5}{|l|}{ Break up/Divorce } \\
\hline \multicolumn{5}{|l|}{ Complicated } \\
\hline relationships & & & & \\
\hline \multicolumn{5}{|l|}{ Achievement/Failure } \\
\hline \multicolumn{5}{|l|}{ Positive } \\
\hline Sports success & & 3 & & \\
\hline Getting into university & & 2 & & 1 \\
\hline \multicolumn{5}{|l|}{ Negative } \\
\hline Not making grades & & & 1 & \\
\hline Failing Exam & & & 1 & \\
\hline \multicolumn{5}{|l|}{ Events/Milestones } \\
\hline \multicolumn{5}{|l|}{ Positive } \\
\hline Good day out & & 1 & & \\
\hline Holiday & & 6 & & \\
\hline Birthday & & 2 & & \\
\hline \multicolumn{5}{|l|}{ Negative } \\
\hline Death of a pet & & & & 1 \\
\hline Going to university & & & & 1 \\
\hline Child leaving school & & & 1 & \\
\hline
\end{tabular}


The negative affect words category was further broken down into sad, angry and anxiety words. A series of one-way ANOVA's (Brown-Forsythe $F$-ratio), revealed significant differences across groups ( $\operatorname{sad}: F(3,24.73)=25.05, p<.001$, anxiety: $F(3,31.12, p=.001$, and angry: $F(3,28.75)=4.52, p=.009)$, although Games-Howell post-hoc analysis found no significant difference in the number of "sad" words used in the control and positive groups. In contrast, memory scripts in the negative group had significantly more "sad" words than the control $(p<.001)$, positive $(p<.001)$ and emotionally confusing groups $(p=.010)$. The emotional confusing group also had significantly more "sad" words than the control $(p<.001)$ and positive groups $(p=$ .001). Post-hoc analysis (Games-Howell) found significantly more "anxiety" words ( $p$ $=.009)$ and "angry" words $(p=.040)$ in the emotionally confusing group compared to control.

Table 12. The mean (SD) percentage of affect words in each group.

\begin{tabular}{lllll}
\hline $\begin{array}{l}\text { Word } \\
\text { Category }\end{array}$ & Control & Positive & Negative & $\begin{array}{l}\text { Emotionally } \\
\text { Confusing }\end{array}$ \\
\hline Affect & $.79(1.58)$ & $6.33(2.84)$ & $8.99(9.52)$ & $7.45(2.49)$ \\
Pos. Affect & $.46(.87)$ & $5.04(2.81)$ & $2.55(3.36)$ & $2.47(1.60)$ \\
Neg. Affect & $.34(.78)$ & $1.24(.99)$ & $6.25(5.98)$ & $4.24(1.99)$ \\
Sad & $.07(.21)$ & $.16(.39)$ & $3.13(2.27)$ & $1.20(.89)$ \\
Anxiety & $.13(.44)$ & $.71(.77)$ & $1.61(2.86)$ & $1.40(1.47)$ \\
Anger & $.03(.14)$ & $.11(.23)$ & $1.16(2.34)$ & $.92(1.30)$ \\
\hline
\end{tabular}

Pos. Affect $=$ positive affect, Neg. Affect $=$ negative affect. 


\subsubsection{Emotionally Confusing Phrases}

As LIWC only analyses individual words, an additional analysis was conducted on the phrases used within the autobiographical recall scripts. This analysis was used to specifically review the scripts from the emotionally confusing group for any additional evidence concerning the effectiveness of participant instructions. It also allowed participants adherence to the mood manipulation protocol to be examined. As a representative example, the following phrases were used by participants when asked to recall a situation when they felt emotionally confused or had experienced difficulty identifying and expressing their emotions:

P6 - "I had a hard time describing my emotions"

P17 - "I find it difficult to express my emotions"

P22 - "Words couldn't express my emotions"

P25 "The combination of emotions all at one time were hard to process"

P67 - "I can't really find any feelings that match words that are right for it"

Such phrases provide additional evidence that participants in the emotionally confusing group were able to successfully follow the novel mood induction paradigm instructions, identifying and thinking about relevant and appropriate past events where they felt a mixture of emotions.

\subsection{Study Three: Discussion}

The overarching aim of Study Three was to develop and test a novel mood manipulation procedure that could effectively induce positive, negative and neural (control) emotional states, as well as induce emotional confusion as an analogue of alexithymia. An autobiographical memory recall procedure was used and for the 
emotionally confusing group, with specific items from the TAS-20 used to generate the wording of the instructions. The utilisation of TAS-20 items aimed to encourage participants to focus on a time they had experienced difficulties identifying and/or describing their emotions. A key objective was to examine the effect of autobiographical recall of positive, negative, neutral or emotionally confusing events on ratings of general mood state as captured by total and subscale scores of the POMS and SME. The positive, negative and control groups were included to check that the emotionally confusing manipulation was tapping into the idea of being unable to identify and/or describe emotions and the experience of feeling emotionally confused specifically, rather than simply generating an overall change in negative mood.

Overall, results revealed that the mood manipulation procedure was successful in eliciting changes in mood and emotional state. Participants in the emotionally confusing group reported feeling less composed and clear-headed, and more emotionally confused, after the mood manipulation. In comparison, participants in the positive and negative groups experienced an increase and decrease in levels of elatedness respectively, with participants in the positive group also experiencing a significant increase in overall mood. In addition, participants in the negative group reported feeling less agreeable following the mood manipulation. Participants in the control group experienced no significant changes in mood from $T_{1}$ to $T_{2}$, highlighting that recalling a journey to work/university was an appropriate control task.

Salas et al. (2012) previously argued that inducing specific emotions via internal mechanisms (i.e. autobiographical recall) may cause other non-target emotions to also increase. The current results provide partial support for this argument, as each mood manipulation induced significant changes in multiple mood states; although these did not cross over across groups. For example, the negative group did 
not elicit the same changes in mood and emotional state observed in the emotional confusing group (e.g. the negative group had a significant reduction in total POMS scores).

Analysis of the self-reported characteristics of recalled autobiographical memories found that a more intense feeling was experienced in the positive, negative and emotional confusing groups compared to the control. Across the three experimental groups, similar levels of intensity were reported, and the tone of the memories recalled was in the desired direction. For example, the negative group reported significantly more negative memories compared to the control, positive and emotional confusing groups. This suggests that the emotionally confusing group induced different changes in mood to the negative group. It may be that for the emotionally confusing group, mixed emotions are experienced with opposite valence, and therefore, are less negative overall.

The number of affect words participants used in their autobiographical scripts also differed significantly across the four groups, providing further support for the validity of the novel mood manipulation paradigm. For instance, participants in the positive group used significantly more positive affect words when describing their chosen memory than those in the control, negative and emotional confusing groups. However, in each group, a wide variety of affect words were used from multiple affect categories, indicating that recalling an emotional event is a complex and dynamic process (Salas et al., 2012). In line with this, when exploring participants autobiographical scripts, Salas and colleagues (2012) found that it was fairly common for different emotions to appear simultaneously. To illustrate, you may initially be scared of something only to subsequently realise that it is harmless. In this scenario, you are experiencing different emotions as the memory unfolds. As a second example, 
you may experience both anger and sadness at particular objects or individuals. As a result, Salas and colleagues (2012) concluded that an individual's emotions may occur in response to their initial emotional state but may change in accordance with the trajectory of the memory recalled. In addition, autobiographical recall is retrospective, and therefore, participants may also include moments of reflection they have had since the event (e.g. an individual feeling more positive about a relationship break-up in the longer-term).

Overall, and consistent with the findings of Appelhans et al. (2011), the current results also suggest that the length of time participants had to complete the manipulation (7 minutes) was sufficient to induce changes in mood and emotional state (e.g. the negative group reported significantly lower levels of agreeableness postmanipulation). However, it could be argued that a significant decrease in overall mood should also have been observed in the negative and emotionally confusing groups. It is possible that the lack of significant reduction in the negative group was due to low overall mood at baseline (19.53) compared to the other groups (30.21-38.06). Although this seems unlikely as baseline mood did not significantly differ statistically across groups (as tested by pairwise comparisons following the 4 x 2 ANOVA). Even so, to ensure that participants are given sufficient opportunity to fully engage with the requirements of the mood manipulation task, it may be beneficial to allow participants a longer time to consider and recall their memories when employing the mood manipulation task in the future. In line with this, task difficulty is also an important consideration. It is possible that recall of an emotionally confusing event is more difficult, and therefore more time-consuming, than the recall of a positive or negative event. However, it appears the participants in the current study managed to complete the emotionally confusing task in the allotted time. Even so, subsequent studies may 
still wish to allow additional time to ensure sufficient opportunity is afforded to participants in all groups to complete the task.

\subsection{Study Three: Conclusion}

Chapter Three presented a review of available mood induction paradigms, focussing specifically on their effectiveness for eliciting changes in mood and emotional state. A novel mood induction paradigm was developed to induce positive, negative and neutral (as a control) emotional states, as well as emotionally confusing feelings as an analogue of alexithymia. Overall, the results from the quantitative and qualitative analyses provide support for the validity and effectiveness of the mood induction paradigm. This novel mood induction paradigm will be utilised in the next chapter to allow examination of the relationship between alexithymia and food intake.

\subsection{Chapter Three: Key Findings}

- Autobiographical recall is an effective method of mood induction within laboratory settings.

- Utilising items from the TAS-20 to guide the induction of emotional confusion as an analogue of alexithymia was successful (e.g. significant decreases in clear-headedness and composure).

- The positive and negative groups produced an increase and decrease in overall mood respectively.

- The negative and emotionally confusing groups differed in their POMS profile after the mood induction. For example, there was a significant reduction in 
clear-headedness and composure scores, and an increase in mixed emotions for the emotionally confusing group.

- A significantly greater number of affect words were used in the autobiographical recall scripts in the positive, negative and emotionally confusing groups compared to control.

- Significantly more positive affect words were used in the positive group, with significantly more negative words used in the negative and emotionally confusing groups.

- The negative and emotionally confusing groups differed significantly from one another, with a greater number of "sad" affect words used in the negative group. 


\section{Chapter Four: The Effects of Mood Manipulation on Food Intake}

\subsection{Introduction}

As set out in the introduction of Chapter Three, the current chapter will explore the associations between alexithymia and actual food intake. This is a logical next step in understanding the mechanisms between alexithymia and emotional eating and a mood manipulation paradigm followed by a bogus taste test was adopted. Previous studies have found mixed results in relation to food intake following a mood manipulation. The current study aimed to advance previous studies by exploring food intake after the induction of an emotionally confusing mood state, as an analogue of alexithymia. This novel manipulation paradigm was tested in Study Three (Chapter Three), where it was found to be a robust and valid method to induce positive and negative mood as well as emotionally confusing states. Chapter Four will begin by reviewing the use of mood induction methods in the context of eating behaviour, and specifically, the effectiveness of mood induction paradigms for manipulating food intake in a laboratory setting. It will then proceed to examine, via the use of the novel mood induction paradigm developed in Chapter Three, the associations between alexithymia and food intake.

\subsection{Mood Induction in Eating Behaviours}

Manipulating an individual's mood to explore its impact on food intake is a common experimental paradigm utilised in eating behaviour research (see Table 13 for examples). As presented in Chapter Three, a wide range of methods can be used to 
induce feelings of positive and negative affect and varying degrees of stress, and evidence suggests that autobiographical recall can be an effective mood manipulation method within eating behaviour research (Appelhans et al., 2011; Evers et al., 2010; Evers et al., 2009; Fay \& Finlayson, 2011; Garg \& Lerner, 2013; Scattolon \& Nicki, 1995; Schneider, Appelhans, \& Whited, 2010; Werthmann et al., 2014; Wilkinson, Rowe, \& Heath, 2013). For example, Appelhans and colleagues (2011) asked participants to focus on a memory of an emotionally intense event for 7 minutes, finding that even though the mood manipulation successfully elicited changes in mood state, food intake did not differ significantly across conditions (anger versus control). Using a similar method, Fay and Finlayson (2011) asked participants to focus on a negative event for 3 minutes. They found that even when participants received little guidance from the experimenter and were afforded only a few minutes to complete the task, a significant decrease in mood occurred after focussing on a negative event compared to completing a control task (recalling daily activities). In contrast to Appelhans et al. (2011), they also found participants consumed more food in the negative condition compared to those in the control condition. Despite mixed results, these previous studies suggest that autobiographical recall may hold significant promise as a mood manipulation method to explore actual behaviour (i.e. food intake) within eating behaviour research.

It is also important to review whether the experimental manipulation of mood more broadly can result in changes in food intake in laboratory settings. Specifically, whether a significant difference in food intake follows an induced emotion compared to a control condition. Overall, whilst the research evidence is mixed, numerous studies have reported a significant increase in food intake following a negative mood induction compared to a neutral/control and/or a positive condition (Chua et al., 2004; 
Dingemans, Martijn, Jansen, et al., 2009; Fay \& Finlayson, 2011; Jansen et al., 2008; Lowe \& Maycock, 1988; Royal \& Kurtz, 2010; Schneider et al., 2010; SheppardSawyer, McNally, \& Fischer, 2000; van Strien et al., 2013; van Strien, Herman, et al., 2012; Wallis \& Hetherington, 2004; see Table 13). As mentioned above, Fay and Finlayson, (2011) found that participants consumed significantly more food after recalling a personal negative event than following recall of daily activities. A strength of this study was the repeated measures design, where all participants completed a control and experimental condition; resulting in each participant acting as their own control. This allowed differences in food intake following the mood induction to be compared to food intake after a control task, after controlling for individual differences. Studies such as Fay and Finlayson (2011), Schneider et al. (2010), Udo et al. (2013) and Wallis and Hetherington (2009), have also adopted similar methods, using each participant as their own control.

Inducing stress specifically, Royal and Kurtz (2010) found that participants who were asked to solve unsolvable anagrams consumed significantly more snacks than participants presented with solvable anagrams. Further analysis found no significant main effect of emotional eating and restraint scores on consumption of snacks across the two groups, although restraint scores were found to significantly mediate the relationship between condition and the number of pieces of food eaten. The authors also reported that both non-emotional eaters in the low stress condition, and high emotional eaters in the high stress condition, overestimated how much they consumed during the anagram task. They concluded that high emotional eaters may overestimate their consumption as a compensatory strategy to prevent them from overeating. That is, if an individual knows from past experience that they have a tendency to overeat during stressful periods, overestimating what they have consumed 
may help the individual to reduce their overall intake. Wallis and Hetherington (2004) also examined the effect of stress on food intake, and in particular, the effect of egothreat. Using a Stroop task, they found that the ego-threat condition (containing words such as "inadequate" and "ridiculed") resulted in a $23 \%$ increase in food intake compared to the usual congruent Stroop task. A $15 \%$ increase in food intake was also seen following the incongruent task, suggesting that whilst an ego-threat can have a large impact on intake, even a slightly cognitive demanding task can have an impact.

However, findings from mood manipulation paradigms are not always straightforward and simple to interpret. For example, within a mood induction paradigm (anger, anxiety, neutral) using a bogus taste test to examine food intake, Schneider et al. (2010) found that high trait anxiety was positively associated with food intake for individuals with obesity, but not for individuals with a lean BMI score. In contrast, trait anger was not significantly associated with food intake for individuals with obesity or a lean BMI score. In addition, Lowe and Maycock (1988) found a significant increase in food intake following a negative mood condition, but only in participants who were sensitive to hunger. Finally, van Strien et al. (2012) found that only individuals who self-reported themselves as high emotional eaters consumed significantly more food following a negative film or stressful task. These studies suggest that although significant differences in food intake were found between mood conditions, additional factors are likely to play a role. Some studies have also reported significant differences in intake between control and experimental conditions, but similar caloric intake in response to positively and negatively evoked emotions (Bongers et al., 2016; Evers et al., 2013).

In addition to negative and stressful mood manipulation, some studies have also found that the induction of a positive mood can result in increased consumption 
of food (Bongers et al., 2013; Cardi et al., 2015; Evers et al., 2009; Macht, Roth, \& Ellgring, 2002; Udo et al., 2013; see Table 13). Evers et al. (2009) conducted a series of small studies and whilst these did not yield significant results, when the studies were combined to increase power they found a significant increase in food consumption following a positive compared to a neutral and negative mood induction. They also concluded that self-reported emotional eating was not associated with food intake in any of the conditions (positive, negative or neutral). Interesting differences have also been found across eating disorders in comparison to healthy controls. Cardi and colleagues (2015) found a significant increase in food intake following a positive induction in individuals with $\mathrm{AN}$, but not in individuals with $\mathrm{BN}$ or healthy controls. However, the latter findings may be due to ceiling effects in the methods (see section 4.3. for further information). Furthermore, Macht et al. (2002) found that males were more likely to feel motivated to consume chocolate following a film clip to induce joy. In addition, males reported the consumption of chocolate to be more enjoyable (greater affect intensity) in the "joy" condition, compared to anger, sadness and fear conditions. In a questionnaire study, Macht (1999) also found that participants reported feeling greater levels of hunger and enjoyment of food when experiencing joy compared to sadness. This suggests that when in a positive mood, the sensations of consuming food are more pleasant and appealing. Therefore, it is likely that individuals are prone to consuming more food when experiencing, or following, a positive mood. This may help to explain why some studies have found increased food intake following positive mood induction.

That said, a decrease in food consumption has also been associated with positive mood. For example, Turner, Luszczynska, Warner, and Schwarzer (2010) found that individuals consumed on average, one less cookie after watching a positive 
film clip compared to those watching a neutral documentary. However, when further examining the relationship between calorie intake and uncontrolled eating style, they found that intake was moderated by experimental condition. Specifically, uncontrolled eaters in the positive condition consumed 3.26 more cookies than uncontrolled eaters in the control condition, providing support for greater consumption following a positive mood for some individuals. They also found no direct effect of self-reported emotional eating on cookie consumption.

Furthermore, it should be noted that some studies have found no significant difference in food consumption following any experimental manipulation of mood (e.g. positive or negative) compared to a neutral/control induction (Appelhans et al., 2011; Haynes et al., 2003; Munsch, Michael, Biedert, Meyer, \& Margraf, 2008; Oliver, Wardle, \& Gibson, 2000; Sproesser, Schupp, \& Renner, 2013; van Strien \& Ouwens, 2007; van Strien, Ouwens, Engel, \& de Weerth, 2014). Although, whilst the majority of such studies have found no significant differences between conditions when simply testing one condition against another, significant differences have emerged when personality traits (e.g. self-reported emotional eating) have been considered. For example, Bongers and colleagues (2013) found no significant differences in food intake across positive, negative and neutral conditions. However, when participants were grouped into self-reported emotional or non-emotional eaters, they found that emotional eaters consumed significantly more food following a positive compared to a negative or neutral mood induction. In contrast, non-emotional eaters consumed similar amounts of food across all three experimental conditions.

Oliver et al. (2000) also found no significant difference in food intake in individuals who were presented with a stressful presentation condition compared to individuals listening to a neutral passage. They also found no significant differences 
in food intake between restraint versus non-restraint, and emotional versus nonemotional eaters. Although, when they compared intake across sweet, salt and bland taste categories, they found that high emotional eaters consumed almost twice as much high fat sweet foods than low emotional eaters during the stress condition. A significant food category $\mathrm{x}$ sex and food category $\mathrm{x}$ fat level interaction was also found. Specifically, males consumed more bland food than females, a finding most apparent for low-fat foods. They also found that males consumed significantly more salty foods than females, a finding that was independent of fat level.

When exploring differences in food intake, two studies have preliminary explored the role of alexithymia (van Strien et al., 2014; van Strien \& Ouwens, 2007). In a sample of healthy females, the number of crackers consumed did not differ significantly across a stress inducing versus control task (van Strien \& Ouwens, 2007), and only scores on the "tension" subscale of the POMS differed significantly from baseline to post-mood manipulation. However, when exploring the possible role of alexithymia, they found that DIF and DDF, both individually and combined, moderated food intake in the distress condition. Participants with higher DIF and DDF scores consumed the same or more in the distress condition despite the 'natural' response to consume less food (van Strien \& Ouwens, 2007). Impulsivity was also tested as a moderator but was not significant. However, it should be noted that the study used the impulse regulation subscale of the Eating Disorders Inventory (EDI-2) which was found to have borderline acceptable levels of internal reliability. In a subsequent study, van Strien et al. (2014) also found that DDF scores were significantly related to food intake in both a stress and neutral condition.

A meta-analysis has also been conducted which can help summarise the literature. Cardi, Leppanen and Treasure (2015) combined the findings of 33 studies, 
concluding that negative mood was significantly associated with greater food intake in comparison to a control condition, and in particular, in individuals with restraint or binge eating tendencies. Positive mood was also associated with greater caloric intake. In addition, more food was also consumed following positive mood induction in comparison to a control. Using a Begg's adjusted rank test (Begg \& Mazumdar, 1994) and Egger's test (Egger, Smith, \& Phillips, 1997), the authors found no evidence of publication bias for food intake between either a negative versus neutral mood induction or between a positive versus neutral mood induction.

Overall, the literature provides mixed support for the consumption of food following a mood induction procedure. Studies have shown significant differences between a control condition and either a manipulated negative or positive mood. However, other studies have also found no significant differences at all. Study Four attempted to clarify the influence of a mood induction procedure on food intake but also expands the literature by adding its own unique and novel element to explore the impact of emotionally confusing emotional states (as an analogue to alexithymia) on food intake.

\subsection{Bogus Taste Test}

When using mood manipulation paradigms to investigate food consumption, how food intake should be measured is an important methodological consideration. One common method is to measure food intake via a bogus taste test (Robinson et al., 2017), which involves presenting participants with one or more food items under the impression that their taste perception is being assessed. The use of a bogus taste test allows for the assessment of food intake across different foods without the bias that 
may arise from self-reports and/or retrospective memories (Guerrieri, Nederkoorn, \& Jansen, 2007; Stice, Fisher, \& Lowe, 2004). A review conducted by Robinson and colleagues (2017) found that bogus taste tests are a valid measure of food intake in the laboratory.

However, when designing bogus taste tests, what food items to present warrants careful consideration. In line with this, food satiety has been found to be reduced when experimenters have used a range of sweet and savoury snacks (Brondel et al., 2009; Norton, Anderson, \& Hetherington, 2006), and when food intake is measured using single foods or meals, no significant differences in consumption have been found (Munsch et al., 2008; S. Schulz \& Laessle, 2012). This indicates that the inclusion of a variety of food items in bogus taste tests is likely to lead to greater consumption, and in turn, allows meaningful differences in consumption across groups to be detected. In support of this, numerous studies have suggested that individuals' food choices change when under stress, with an increased tendency to consume sugary, high salt and fatty foods, most often snack foods, observed (Cartwright et al., 2003; Gibson, 2006; Laitinen, Ek, \& Sovio, 2002; Michaud et al., 1990; Nguyen-Michel, Unger, \& Spruijt-Metz, 2007; Snoek, van Strien, Janssens, \& Engels, 2006; Wardle et al., 2000). Therefore, choice of food is an important methodological consideration when designing experimental studies involving a bogus taste test design (Oliver et al., 2000). Furthermore, research has shown that emotional eating occurs through snacking rather than increased intake during meals (Baumeister, Heatherton, \& Tice, 1994; Oliver \& Wardle, 1999). Consequently, conducting studies using food items most commonly associated with emotional eating brings further validity to obtained results. In addition to variety, a further factor to consider when designing a bogus taste test is how palatable foods are and how much they are liked by participants. One 
method is to ask participants to rate the palatability of a range of snack foods (e.g. peanut butter cups, crisps, chocolate chip cookies and ice cream) in a screening session and to then present the participants with snacks they rated as being highly palatable (Appelhans et al., 2011). However, even when the number of calories presented to each participant is standardised, it ultimately means that there is no standardised set of snack foods across participants. Further, Appelhans and colleagues (2011) argue that although such an approach would reduce extraneous variables associated with taste preferences and increase external validity as a result, it would ultimately cause differences in overall presentation of foods. In their study, Appelhans and colleagues highlighted how a 400kcal portion of crisps looks visually different (bigger or smaller) than a 400kcal portion of cookies, which may impact the amount of food consumed. They also acknowledged that the inclusion of food such as ice cream may also influence consumption. Here, participants might consume all the ice cream to avoid waste as it melts or not consume as much as they wanted as it would have been more difficult to eat compared with crisps or cookies. Food items such as crisps or cookies that come as 'whole' are also important to consider as participants may decide to eat a set amount of each item, whereas if they are broken up, they do not know how much they are consuming and cannot follow any rules they may have about consuming snacks (Higgs \& Donohoe, 2011; Jansen et al., 2008).

Another consideration when choosing food items is the possibility of ceiling effects arising from the portion of food or drink that is presented to participants. As an example, Cardi et al. (2015) used a standardised $250 \mathrm{ml}$ portion of smoothie drink and found no significant difference in consumption across a neutral and negative condition in individuals with $\mathrm{BN}$ and a healthy control group. However, this finding may be 
attributable to some participants in each condition consuming all of the smoothie drink, making it difficult to distinguish results, especially at the higher end of consumption.

Finally, it is also important to think about the instructions given to participants and the environment they are left in to engage with the taste test. In a participant level analysis, Robinson and colleagues (2017) found that participants were usually left for ten minutes. It is also best practice to leave participants alone during a taste test (e.g. Evers et al., 2009; Guerrieri et al., 2007) so they do not feel scrutinised or under pressure to perform and/or respond in a particular way. Bongers et al. (2013) also suggested that a non-eating condition should be included to ensure that any consumption of food occurs in response to the manipulation itself rather than as a way of passing time. Some studies (e.g. Moon \& Berenbaum, 2009) do not involve a taste test, and instead food is left in the room as a 'thank you' or offered without full instructions. Here, participants are left alone to complete another task and so the food may be consumed as a way of passing time. However, Evers and colleagues (2018) stated that irrespective of how long participants are given, the time constraints within a laboratory setting prevent capturing a participant's reaction to a situation (e.g. negative mood induction). Instead, the time constraint limits studies of mood induction and food intake to a snapshot of a participant's behaviour. Nevertheless, the authors also acknowledged that validly tracking an individual's food consumption over time is difficult and laboratory-based studies are an important tool. In terms of instructions, participants are often given a food perception questionnaire to complete during the taste test that focuses them on to the food and to ensure that they try all of the foods at least once (e.g. Evers et al., 2009; Evers et al., 2010). It is often common to tell participants that they can eat as much or as little as they like (e.g. Evers et al., 2009; Evers et al., 2010; Fay \& Finlayson, 2011; Royal \& Kurtz, 2010) or to facilitate 
consumption by informing participants that any left-over food will be discarded following the session (e.g. Appelhans et al., 2011).

To conclude, when designing a bogus taste test, variety, palatability, portion size and the instructions given to participants are all important methodological factors to consider. The sample of sweet and savoury snacks used in the current study have previously been sampled in a study within the Swansea Nutrition, Appetite and Cognition (SNAC) group and found to be sufficient in providing a measure of food intake (Price, Lee, \& Higgs, 2016). Price and colleagues (2016) found that a high construal prime significantly reduced food intake (small-moderate effect size) when combined with a reminder cue versus no cue and a low construal prime.

Table 13 presents an overview of mood induction and food intake literature. Significant main effects of mood and food intake are indicated by either $\checkmark$ or $X$. That is, if significant changes in mood were observed following the induction of a particular mood sate, this will be marked as $\checkmark$ and non-significant changes with $X$. Studies that did not test for a main effect of mood on food intake without additional personality factors, e.g. self-reported emotional eating, alexithymia are indicated with an Asterix $(*)$ 
Table 13. Overview of mood induction and food intake studies.

\begin{tabular}{|c|c|c|c|c|c|c|}
\hline \multirow[t]{2}{*}{ Study } & \multirow[t]{2}{*}{ Sample } & \multirow[t]{2}{*}{$\begin{array}{c}\text { Mood } \\
\text { Manipulation }\end{array}$} & \multirow[t]{2}{*}{ Food Task } & \multicolumn{2}{|c|}{$\begin{array}{l}\text { Significant } \\
\text { difference }\end{array}$} & \\
\hline & & & & Mood & Intake* & \\
\hline $\begin{array}{l}\text { Appelhans et } \\
\text { al. (2011) }\end{array}$ & $\begin{array}{l}37 \text { lean }(\mathrm{F}=24) \\
\text { adults } \\
\text { Age: } 31.8 \text { years } \\
(10.6) \\
\text { BMI }=22.8 \\
(1.7) \\
24 \text { obese }(\mathrm{F}= \\
21) \text { adults } \\
\text { Age: } 39.0 \text { years } \\
(11.4) \\
\text { BMI: } 34.9(4.0)\end{array}$ & $\begin{array}{l}\text { Autobiographica } \\
1 \text { recall: pre- } \\
\text { study interview } \\
\text { and highest } \\
\text { rated memory } \\
\text { used. Negative = } \\
\text { anger, and } \\
\text { neutral = } \\
\text { household task. } \\
\text { Measure: POMS }\end{array}$ & $\begin{array}{l}\text { Mixed snacks: } \\
6 / 38 \\
\text { predetermined } \\
\text { e.g. peanut } \\
\text { butter cups, } \\
\text { pretzels, chips, } \\
\text { cookies. } \\
\text { Measure: } \\
\text { caloric value. }\end{array}$ & $\checkmark$ & $x$ & $\begin{array}{l}\text { Mood manipul } \\
\text { ratings signific } \\
\text { post-manipulat } \\
\text { No significant } \\
\text { consumption } \\
\text { Significant BM } \\
\text { distraction sco } \\
\text { consumption in } \\
\text { in individuals } ~\end{array}$ \\
\hline $\begin{array}{l}\text { Bekker, van } \\
\text { de } \\
\text { Meerendonk, } \\
\& \text { Mollerus } \\
(2004)\end{array}$ & $\begin{array}{l}52 \text { females } \\
\text { EE: DEBQ } \\
\text { average range } \\
33.38-39.00 \\
\text { Split into high } \\
\text { and low } \\
\text { impulsivity } \\
\text { (BIS-11 Med. = } \\
66)\end{array}$ & $\begin{array}{l}\text { Stress } \\
\text { manipulation: } \\
\text { negative = } \\
\text { difficult quiz, } \\
\text { and control = } \\
\text { easy quiz. } \\
\text { Measure: } \\
\text { POMS. }\end{array}$ & $\begin{array}{l}\text { Mixed snacks: } \\
\text { sweets and } \\
\text { salty biscuits. } \\
\text { Measure: N/A. }\end{array}$ & $\checkmark$ & $\sqrt{ }^{+}$ & $\begin{array}{l}\text { Mood manipu } \\
\text { negative affec } \\
\text { condition. } \\
\text { Significant mai } \\
\text { emotional eati } \\
\text { condition. } \\
\text { impulsivity int } \\
\text { main effect of }\end{array}$ \\
\hline $\begin{array}{l}\text { Bongers et al. } \\
\text { (2013) }\end{array}$ & $\begin{array}{l}86 \text { females } \\
\text { Age: } 21.6 \text { years } \\
(2.8)\end{array}$ & $\begin{array}{l}\text { Film excerpts: } \\
\text { positive = "Mr } \\
\text { Bean" and } \\
\text { "When Harry } \\
\text { met Sally", }\end{array}$ & $\begin{array}{l}\text { Mixed snacks: } \\
\text { crisps and } \\
\text { chocolate. } \\
\text { Measure: } \\
\text { caloric value. }\end{array}$ & $\checkmark$ & $x$ & $\begin{array}{l}\text { Mood manipu } \\
\text { condition beca } \\
\text { mood and neg: } \\
\text { significant char }\end{array}$ \\
\hline
\end{tabular}




\begin{tabular}{|c|c|c|c|c|c|c|}
\hline & $\begin{array}{l}\text { BMI: average } \\
\text { range }=23.10- \\
23.89 \\
\text { EE: DEBQ } \\
\text { average range = } \\
2.13-2.38\end{array}$ & $\begin{array}{l}\text { negative }=\text { "The } \\
\text { Green Mile" and } \\
\text { neutral = } \\
\text { "fishing } \\
\text { documentary". } \\
\text { Measure: VAS. }\end{array}$ & & & & $\begin{array}{l}\text { No significant } 1 \\
\text { intake across g } \\
\text { trend-significar } \\
\text { eaters in the po } \\
\text { found in the } \\
\text { eaters consumi } \\
\text { observed in the }\end{array}$ \\
\hline $\begin{array}{l}\text { Bongers et al. } \\
\text { (2016) }\end{array}$ & $\begin{array}{l}42 \text { females } \\
\text { Age: } 20.26 \\
(1.82) \\
\text { BMI: } 21.83 \\
(2.14) \\
\text { EE: DEBQ: } 2.46 \\
(.68)\end{array}$ & $\begin{array}{l}\text { Mixed: negative } \\
=\text { sad music }+ \\
\text { sad memory and } \\
\text { positive = happy } \\
\text { music and happy } \\
\text { memory and } \\
\text { control = dot to } \\
\text { do puzzles. } \\
\text { Measure: VAS. }\end{array}$ & $\begin{array}{l}\text { Mixed snacks: } \\
\text { predetermined } \\
\text { by participants, } \\
\text { e.g. chocolate, } \\
\text { crisps, cookies, } \\
\text { peanuts. } \\
\text { Measure: } \\
\text { caloric value. }\end{array}$ & $\checkmark$ & * & $\begin{array}{l}\text { Mood manipu } \\
\text { increase in "sac } \\
\text { for the sad con } \\
\text { condition. No } \\
\text { condition. } \\
\text { Food intake } \\
\text { significantly re } \\
\text { control conditic } \\
\text { conditions for } \\
\text { eaters showed } \\
\text { across conditio } \\
\text { scores signific } \\
\text { intake in all col }\end{array}$ \\
\hline $\begin{array}{l}\text { Cardi et al. } \\
\text { (2015) }\end{array}$ & $\begin{array}{l}19 \text { females with } \\
\text { AN. Age: } 31.0 \\
\text { years (10), BMI: } \\
16.7(2.7) \\
23 \text { females with } \\
\text { BN. Age: } 24.4 \\
\text { years (5.7), } \\
\text { BMI: } 23.4(6.9)\end{array}$ & $\begin{array}{l}\text { Vodcast: } \\
\text { positive = } \\
\text { uplifting music } \\
\text { with positive } \\
\text { statements and } \\
\text { neutral = } \\
\text { instrumental } \\
\text { music with facts }\end{array}$ & $\begin{array}{l}\text { Drink: Ps } \\
\text { selected } 1 \text { of } 3 \\
\text { smoothies; } \\
\text { strawberry and } \\
\text { banana, mango } \\
\text { and } \\
\text { passionfruit, or } \\
\text { kiwi, apple and } \\
\text { lime. }\end{array}$ & $\sqrt{ }^{+}$ & $\checkmark$ & $\begin{array}{l}\text { Mood manipu } \\
\text { participants rep } \\
\text { positive affect } \\
\text { +but it approac } \\
\text { and BN ( } p= \\
\text { healthy control } \\
\text { negative affect } \\
\text { The AN group } \\
\text { controls consu }\end{array}$ \\
\hline
\end{tabular}




\begin{tabular}{|c|c|c|c|c|c|c|}
\hline & $\begin{array}{l}36 \text { healthy } \\
\text { control females. } \\
\text { Age: } 25.9 \text { years } \\
(5.0), \text { BMI: } 21.5 \\
(2.0) \text {. }\end{array}$ & $\begin{array}{l}\text { and neutral } \\
\text { statements. } \\
\text { Measure: } \\
\text { PANAS and } \\
\text { VAS. }\end{array}$ & $\begin{array}{l}\text { Measure: } \\
\text { caloric value. }\end{array}$ & & & $\begin{array}{l}\text { consumed sig } \\
\text { condition than } \\
\text { no significant } d \\
\text { or healthy cont }\end{array}$ \\
\hline $\begin{array}{l}\text { Chua et al. } \\
\text { (2004) }\end{array}$ & $\begin{array}{l}40 \text { females with } \\
\text { obesity. } \\
\text { Age: } 41.7 \text { years } \\
\text { (21-64 years) } \\
\text { BMI: } 40.2 \\
(28.9-54.6)\end{array}$ & $\begin{array}{l}\text { Film excerpts: } \\
\text { negative = } \\
\text { "Gale is Dead" } \\
\text { and neutral = } \\
\text { "Bali } \\
\text { documentary." } \\
\text { Measure: VAS. }\end{array}$ & $\begin{array}{l}\text { Sweet snacks: } \\
\text { Chocolate: } \\
\text { white, dark, } \\
\text { milk and fruit } \\
\text { and nut. } \\
\text { Measure: } \\
\text { grams. }\end{array}$ & $\checkmark$ & $\checkmark$ & $\begin{array}{l}\text { Mood manipu } \\
\text { condition sign } \\
\text { and decreased } \\
\text { Negative cond } \\
\text { than neutral co } \\
\text { restraint, but } \\
\text { negative con } \\
\text { individuals in } t\end{array}$ \\
\hline $\begin{array}{l}\text { Dingemans et } \\
\text { al. (2009) }\end{array}$ & $\begin{array}{l}66 \text { females with } \\
\text { BED } \\
\text { Age: } 39 \text { years } \\
\text { (9.6) } \\
\text { BMI: average } \\
\text { range = 32.6- } \\
33.8\end{array}$ & $\begin{array}{l}\text { Film excerpts: } \\
\text { negative = "The } \\
\text { Champ" } \\
\text { followed by } \\
\text { instruction to } \\
\text { suppress } \\
\text { emotions or } \\
\text { react naturally. } \\
\text { Measure: VAS. }\end{array}$ & $\begin{array}{l}\text { Mixed snacks: } \\
\text { Chocolate, } \\
\text { potato chips, } \\
\text { marshmallows } \\
\text { and cake. } \\
\text { Measure: } \\
\text { caloric value. }\end{array}$ & $\checkmark$ & $*$ & $\begin{array}{l}\text { Mood manipula } \\
\text { significantly m } \\
\text { film. } \\
\text { Suppression co } \\
\text { the natural re: } \\
\text { condition } \mathrm{x} \text { le } \\
\text { more depresse } \\
\text { more their m } \\
\text { manipulation, } t\end{array}$ \\
\hline $\begin{array}{l}\text { Dingemans, } \\
\text { Martijn, } \\
\text { Furth, \& } \\
\text { Jansen (2009) }\end{array}$ & $\begin{array}{l}73 \text { females with } \\
\text { BED } \\
\text { Age: } 18-60 \\
\text { years } \\
\text { BMI: } 34.7(7.8)\end{array}$ & $\begin{array}{l}\text { Film excerpts; } \\
\text { negative = "The } \\
\text { Champ", } \\
\text { positive = } \\
\text { "When Harry } \\
\text { met Sally." } \\
\text { Food-mood }\end{array}$ & $\begin{array}{l}\text { Mixed snacks: } \\
\text { chocolate, } \\
\text { potato chips, } \\
\text { candy and } \\
\text { cake. } \\
\text { Measure: } \\
\text { caloric value. }\end{array}$ & $\checkmark$ & $x$ & $\begin{array}{l}\text { Mood manipul } \\
\text { were successf } \\
\text { negative and } \\
\text { Confirmation } \\
\text { emotions were } \\
\text { No significant } \\
\text { or food x mood }\end{array}$ \\
\hline
\end{tabular}




\begin{tabular}{|c|c|c|c|c|c|c|}
\hline & & $\begin{array}{l}\text { expectation } \\
\text { manipulation; } \\
\text { confirmation } \\
\text { versus } \\
\text { disconfirmation. } \\
\text { Measure: VAS. }\end{array}$ & & & & $\begin{array}{l}\text { consumed acr } \\
\text { caloric intake } r \\
\text { pleasurable anc } \\
\text { In the negati } \\
\text { no/mild depres } \\
\text { moderate/sever }\end{array}$ \\
\hline $\begin{array}{l}\text { Emery, King, } \\
\text { \& Levine } \\
(2014)\end{array}$ & $\begin{array}{l}106 \text { females } \\
\text { Age: } 18-25 \\
\text { years }\end{array}$ & $\begin{array}{l}\text { Stress } \\
\text { manipulation: } \\
\text { control = } \\
\text { untimed puzzle } \\
\text { and supportive } \\
\text { instructions and } \\
\text { experimental = } \\
\text { timed puzzle } \\
\text { with negative } \\
\text { feedback. } \\
\text { Measure: } \\
\text { PANAS }\end{array}$ & $\begin{array}{l}\text { Mixed snacks: } \\
\text { Skittles®, M \& } \\
\text { M's® and } \\
\text { Ritz® } \\
\text { crackers. } \\
\text { Measure: } \\
\text { caloric value. }\end{array}$ & $\checkmark$ & $*$ & $\begin{array}{l}\text { Mood manipul } \\
\text { greater negati } \\
\text { compared to co } \\
\text { Negative urgen } \\
\text { dietary restrai } \\
\text { restraint and } 1 \\
\text { fewer calories } \\
\text { affect enhance } \\
\text { negative urgen } \\
\text { among individ }\end{array}$ \\
\hline $\begin{array}{l}\text { Evers et al. } \\
(2009)\end{array}$ & $\begin{array}{l}\text { Study } 1 \\
30 \text { females. } \\
\text { Age: } 21.8 \text { years } \\
(21.74) . \\
\text { BMI: } 21.74 \\
(2.4) \\
\text { EE: DEBQ } 2.75 \\
(.59)\end{array}$ & $\begin{array}{l}\text { Vignettes: } \\
\text { negative = main } \\
\text { character in a } \\
\text { story where the } \\
\text { mother suddenly } \\
\text { died. } \\
\text { Measure: } \\
\text { negative and } \\
\text { positive } \\
\text { compounds. }\end{array}$ & $\begin{array}{l}\text { Mixed snacks: } \\
\text { Chocolate, } \\
\text { crisps, raisins, } \\
\text { and crackers. } \\
\text { Measure: } \\
\text { caloric value. }\end{array}$ & $\checkmark$ & $*$ & $\begin{array}{l}\text { Mood manipu } \\
\text { emotions inc } \\
\text { decreased follo } \\
\text { Emotional and } \\
\text { similar amoun } \\
\text { manipulation. }\end{array}$ \\
\hline
\end{tabular}




\begin{tabular}{|c|c|c|c|c|c|}
\hline $\begin{array}{l}\text { Study } 2 \\
60 \text { females. } \\
\text { Age: } 20.9 \text { years } \\
\text { BMI: } 21.49 \\
(2.39) \\
\text { EE: DEBQ } 2.67 \\
(.50)\end{array}$ & $\begin{array}{l}\text { Film excerpts: } \\
\text { positive = e.g. } \\
\text { "When Harry } \\
\text { met Sally" and } \\
\text { negative = e.g. } \\
\text { "Blair Witch } \\
\text { Project." } \\
\text { Measure: } \\
\text { negative and } \\
\text { positive } \\
\text { compounds. }\end{array}$ & $\begin{array}{l}\text { Mixed snacks: } \\
\text { Chocolate, } \\
\text { crisps, fruit } \\
\text { and crackers. } \\
\text { Measure: } \\
\text { caloric value. }\end{array}$ & $\sqrt{ }$ & $x$ & $\begin{array}{l}\text { Mood manipu. } \\
\text { increase in ne } \\
\text { emotion in the } \\
\text { was for the pos } \\
\text { No significant } \\
\text { the positive an } \\
\text { and non-emotic }\end{array}$ \\
\hline $\begin{array}{l}\text { Study } 3 \\
37 \text { females } \\
\text { Age: } 22.84 \\
\text { years } \\
\text { BM: } 22.9 \text { (2.97) } \\
\text { EE: DEBQ } 2.82 \\
(.65)\end{array}$ & $\begin{array}{l}\text { Autobiographica } \\
1 \text { recall: negative } \\
=\text { personally sad } \\
\text { event and } \\
\text { neutral = daily } \\
\text { event. } \\
\text { Measure: } \\
\text { negative and } \\
\text { positive } \\
\text { compounds. }\end{array}$ & $\begin{array}{l}\text { Mixed snacks: } \\
\text { Chocolate, } \\
\text { crisps, and } \\
\text { cookies. } \\
\text { Measure: } \\
\text { caloric value. }\end{array}$ & $\checkmark$ & $x$ & $\begin{array}{l}\text { Mood manipu } \\
\text { condition sig } \\
\text { emotions and } \\
\text { significant char } \\
\text { Emotional eate } \\
\text { more than non- } \\
\text { significant diff } \\
\text { negative and ne }\end{array}$ \\
\hline $\begin{array}{l}\text { Study } 4 \\
57 \text { lean females } \\
\text { Age: } 20.8 \text { years } \\
\text { (NR) } \\
\text { BMI: } 21.80 \\
\text { (3.46). } \\
\text { EE: DEBQ } 2.74\end{array}$ & $\begin{array}{l}\text { False feedback: } \\
\text { positive = } \\
\text { positive ratings, } \\
\text { negative = } \\
\text { negative ratings, } \\
\text { and neutral = } \\
\text { told feedback }\end{array}$ & $\begin{array}{l}\text { Mixed snacks: } \\
\text { Chocolate, } \\
\text { crisps, fruit } \\
\text { and crackers. } \\
\text { Measure: } \\
\text { caloric value. }\end{array}$ & $\checkmark$ & $x$ & $\begin{array}{l}\text { Mood manil } \\
\text { condition siq } \\
\text { decreased p } \\
\text { found in } p \\
\text { changes in ne } \\
\text { Emotional ec } \\
\text { more than no }\end{array}$ \\
\hline
\end{tabular}


would happen

later.

Measure:

negative and

positive

compounds. significant diff negative and $n$
Study 5

Merged studies

1-4.

EE: DEBQ 2.74

(2.85) $\checkmark \begin{array}{lll}\checkmark & \checkmark & \text { Mood manipu }\end{array}$

above.

No significant emotional and Positive condil than negative $c$ difference bet condition.

Mood manipu. increase in "sa compared to th No significan conditions. $\mathrm{H}$ associated with condition. Mood and successful: increased foll participants er instructed to th Greater intake condition vers 


\begin{tabular}{|c|c|c|c|c|c|}
\hline & $\begin{array}{l}\text { Measure: } 21 \\
\text { emotions rated. }\end{array}$ & $\begin{array}{l}\text { Measure: } \\
\text { grams }\end{array}$ & & & $\begin{array}{l}\text { significant effe } \\
\text { and non-comfo }\end{array}$ \\
\hline $\begin{array}{l}\text { Study } 3 \\
62 \text { females } \\
\text { Age: } 21.5 \text { years } \\
\text { BMI: } 22.46 \\
(2.85)\end{array}$ & $\begin{array}{l}\text { Film excerpt: } \\
\text { negative = } \\
\text { "American } \\
\text { History X" } \\
\text { Plus, } \\
\text { suppression, } \\
\text { reappraisal and } \\
\text { control. } \\
\text { Measure: } 21 \\
\text { emotions rated. }\end{array}$ & $\begin{array}{l}\text { Mixed snacks: } \\
\text { unhealthy = } \\
\text { crisps and } \\
\text { chocolate, and } \\
\text { healthy = } \\
\text { salted and } \\
\text { unsalted } \\
\text { crackers } \\
\text { Measure: } \\
\text { grams }\end{array}$ & $\checkmark$ & * & $\begin{array}{l}\text { Mood and } \\
\text { successful: } \mathrm{n} \\
\text { increased foll } \\
\text { participants er } \\
\text { instructed to th } \\
\text { Greater intake } \\
\text { condition com } \\
\text { Reappraisal di } \\
\text { significant effe }\end{array}$ \\
\hline $\begin{array}{l}\text { Study } 1 \\
16 \text { males, } 52 \\
\text { females } \\
\text { Age: } 21.9 \text { years } \\
(3.3) \\
\text { BMI: } 21.5 \\
(1.95)\end{array}$ & $\begin{array}{l}\text { Video clips: } \\
\text { positive = e.g. } \\
\text { panda sneezing } \\
\text { and control = } \\
\text { birds in the } \\
\text { desert. } \\
\text { Measure: } 19 \\
\text { emotions from } \\
\text { the post film } \\
\text { questionnaire. }\end{array}$ & $\begin{array}{l}\text { Mixed snacks: } \\
\text { M \& M's®, } \\
\text { coated peanuts } \\
\text { and wine } \\
\text { gums. } \\
\text { Measure: } \\
\text { caloric intake. }\end{array}$ & $\checkmark$ & $\sqrt{ }$ & $\begin{array}{l}\text { Mood manipul } \\
\text { increased posit } \\
\text { in the positive } \\
\text { significantly de } \\
\text { change to posit } \\
\text { Significant ma } \\
\text { condition cons } \\
\text { snacks. Dietar } \\
\text { relationship } \\
\text { consumption. }\end{array}$ \\
\hline $\begin{array}{l}\text { Study } 2 \\
84 \text { females } \\
\text { BMI: } 22.35 \\
(2.48)\end{array}$ & $\begin{array}{l}\text { Autobiographica } \\
1 \text { recall: positive } \\
=\text { pleasant event, } \\
\text { negative = sad } \\
\text { event and } \\
\text { control = daily } \\
\text { activity. }\end{array}$ & $\begin{array}{l}\text { Mixed snacks: } \\
\text { chocolate, } \\
\text { crisps, biscuits } \\
\text { and crackers. } \\
\text { Measure: } \\
\text { caloric intake. }\end{array}$ & $\checkmark$ & $\sqrt{ }$ & $\begin{array}{l}\text { Mood manipul } \\
\text { increase in } \mathrm{pc} \\
\text { emotions for } \mathrm{p} \\
\text { for the negati } \\
\text { control conditi } \\
\text { Significant mai } \\
\text { negative condi }\end{array}$ \\
\hline
\end{tabular}




\begin{tabular}{|c|c|c|c|c|c|c|}
\hline & $\begin{array}{l}\text { Age: average } \\
\text { range }=19.6- \\
26.2 \text { years } \\
\text { BMI: average } \\
\text { range }=21.6- \\
23.8\end{array}$ & $\begin{array}{l}\text { on the task plus } \\
\text { mental } \\
\text { arithmetic, and } \\
\text { no-stress = } \\
\text { correct } \\
\text { feedback. } \\
\text { Measure: POMS }\end{array}$ & $\begin{array}{l}\text { egg } \\
\text { sandwiches, } \\
\text { cheese cubes, } \\
\text { cherry } \\
\text { tomatoes, } \\
\text { potato crisps } \\
\text { cake, chocolate } \\
\text { and biscuits. } \\
\text { Measure: } \\
\text { calorie value }\end{array}$ & & & $\begin{array}{l}\text { affect in the stre } \\
\text { stress condition } \\
\text { In the stress } \\
\text { similar amount } \\
\text { the stress condi } \\
\text { group also con } \\
\text { whereas LRHI } \\
\text { differences for }\end{array}$ \\
\hline $\begin{array}{l}\text { Jansen et al. } \\
(2008)\end{array}$ & $\begin{array}{l}53 \text { females with } \\
\text { overweight and } \\
\text { obesity } \\
\text { Age: } 41.0 \text { years } \\
\text { (6.3) } \\
\text { BMI: } 36.4(6.0) \\
38 \text { age matched } \\
\text { healthy controls } \\
\text { Age: } 41.6 \text { years } \\
\text { (7.6) } \\
\text { BMI: } 22.0(2.0)\end{array}$ & $\begin{array}{l}\text { Mixed: neutral = } \\
\text { festival parade } \\
\text { video, negative } \\
\text { = piece of music } \\
\text { and thoughts of } \\
\text { an unpleasant } \\
\text { event in their } \\
\text { past, and food } \\
\text { exposure = } \\
\text { smelling the } \\
\text { foods. } \\
\text { Measure: POMS }\end{array}$ & $\begin{array}{l}\text { Mixed snacks: } \\
\text { chocolate, } \\
\text { savoury nuts, } \\
\text { cookies and } \\
\text { chocolate and } \\
\text { vanilla } \\
\text { milkshakes. } \\
\text { Measure: } \\
\text { caloric value }\end{array}$ & $\checkmark$ & $x$ & $\begin{array}{l}\text { Mood manipu } \\
\text { successful: incr } \\
\text { food exposure } \\
\text { significantly d } \\
\text { neutral conditi } \\
\text { with no change } \\
\text { No significant } \\
\text { condition but t } \\
\text { condition x ne } \\
\text { Participants wi } \\
\text { consumed more } \\
\text { food exposure, } \\
\text { not. Participan } \\
\text { similar amount }\end{array}$ \\
\hline $\begin{array}{l}\text { Lowe \& } \\
\text { Maycock } \\
(1988)\end{array}$ & $\begin{array}{l}60 \text { female } \\
\text { students all < } \\
15 \% \text { overweight }\end{array}$ & $\begin{array}{l}\text { Velten: } \\
\text { depressed = } \\
\text { increasing } \\
\text { depressing self- } \\
\text { statement, and }\end{array}$ & $\begin{array}{l}\text { Sweet snack: } \\
\text { M \& M's }{ }^{\circledR} \\
\text { Measure: } \\
\text { grams }\end{array}$ & $\checkmark$ & $*$ & $\begin{array}{l}\text { Mood manipul } \\
\text { reported great } \\
\text { depressed cor } \\
\text { condition. }\end{array}$ \\
\hline
\end{tabular}




\begin{tabular}{|c|c|c|c|c|c|c|}
\hline & & $\begin{array}{l}\text { neutral = bland } \\
\text { descriptive } \\
\text { statements. } \\
\text { Measure: PFS } \\
\text { and MAACL-D }\end{array}$ & & & & $\begin{array}{l}\text { No significant } \\
\text { disinhibition, } \\
\text { hunger sensitiv } \\
\text { interaction. In } \\
\text { group consume } \\
\text { group. }\end{array}$ \\
\hline $\begin{array}{l}\text { Macht et al. } \\
(2002)\end{array}$ & $\begin{array}{l}48 \text { males } \\
\text { Age: } 28 \text { years } \\
(6.55) \\
\text { BMI: } 23.2(2.4)\end{array}$ & $\begin{array}{l}\text { Film excerpts; } \\
\text { anger = "Cry } \\
\text { Freedom", fear } \\
=\text { "Silence of the } \\
\text { Lambs", sad = } \\
\text { "The Champ", } \\
\text { and joy = "When } \\
\text { Harry met } \\
\text { Sally" } \\
\text { Measure: VAS }\end{array}$ & $\begin{array}{l}\text { Sweet snack: } \\
\text { chocolate } \\
\text { Measure: N/A. }\end{array}$ & $\sqrt{ }^{+}$ & $N / A$ & $\begin{array}{l}\text { Mood manipu } \\
\text { changes in moc } \\
\text { changes were } r \\
\text { each condition. } \\
\text { Desire to eat } w \\
\text { condition and il } \\
\text { intense affectiv } \\
\text { condition comp } \\
\text { and more inten } \\
\text { sadness and an }\end{array}$ \\
\hline $\begin{array}{l}\text { Moon \& } \\
\text { Berenbaum } \\
(2009)\end{array}$ & $\begin{array}{l}100 \text { female } \\
\text { students } \\
\text { Age: } 18.7 \text { years } \\
(.8)\end{array}$ & $\begin{array}{l}\text { Social } \\
\text { evaluation: all } \\
\text { participants. } \\
\text { High and low } \\
\text { emotional } \\
\text { attention } \\
\text { conditions. } \\
\text { Measure: N/A. }\end{array}$ & $\begin{array}{l}\text { Mixed snacks: } \\
\text { potato chips } \\
\text { and cookies. } \\
\text { Measure: } \\
\text { caloric value }\end{array}$ & - & $\checkmark$ & $\begin{array}{l}\text { No manipulatic } \\
\text { Low attention } \\
\text { emotion bot } \\
\text { consumption. I } \\
\text { trait attention } \\
\text { consumption b } \\
\text { trait attention } \\
\text { differ. }\end{array}$ \\
\hline $\begin{array}{l}\text { Munsch et al. } \\
\text { (2008) }\end{array}$ & $\begin{array}{l}69 \text { females with } \\
\text { overweight and } \\
\text { obesity with } \\
\text { BED }\end{array}$ & $\begin{array}{l}\text { Vivid guided } \\
\text { imagery: } \\
\text { negative = } \\
\text { idiosyncratic } \\
\text { stressor, and }\end{array}$ & $\begin{array}{l}\text { Sweet meal: } \\
\text { dessert cremes; } \\
\text { vanilla, } \\
\text { chocolate and } \\
\text { caramel. }\end{array}$ & $\checkmark$ & $x$ & $\begin{array}{l}\text { Mood manipul } \\
\text { condition cause } \\
\text { affect and ten } \\
\text { condition. }\end{array}$ \\
\hline
\end{tabular}




\begin{tabular}{|c|c|c|c|c|c|c|}
\hline & $\begin{array}{l}\text { Age: average } \\
\text { range }=42.53- \\
49.71 \\
\text { BMI: average } \\
\text { range }=32.48- \\
33.68\end{array}$ & $\begin{array}{l}\text { neutral = boat } \\
\text { anchoring in a } \\
\text { harbour. } \\
\text { Measure: VAS }\end{array}$ & $\begin{array}{l}\text { Measure: } \\
\text { grams }\end{array}$ & & & $\begin{array}{l}\text { The mood man } \\
\text { or hunger. No } s \\
\text { between the th } \\
\text { or interaction } \mathrm{e}\end{array}$ \\
\hline $\begin{array}{l}\text { Oliver et al. } \\
(2000)\end{array}$ & $\begin{array}{l}27 \text { healthy } \\
\text { males and } 41 \\
\text { healthy females } \\
\text { Age: average } \\
\text { range = } 25.3- \\
26.9 \text { years } \\
\text { BMI: average } \\
\text { range = } 21.7- \\
23.1 \\
\text { EE: DEBQ } \\
\text { average range = } \\
2.18-3.07\end{array}$ & $\begin{array}{l}\text { Stress } \\
\text { manipulation: } \\
\text { negative = } \\
\text { controversial } \\
\text { speech } \\
\text { performance and } \\
\text { neutral = listen } \\
\text { to text excerpt } \\
\text { (“Under milk } \\
\text { wood") } \\
\text { Measure: } \\
\text { PANAS }\end{array}$ & $\begin{array}{l}\text { Mixed snacks: } \\
34 \text { foods with } \\
\text { a range of high } \\
\text { and low fat } \\
\text { salty, sweet } \\
\text { and bland } \\
\text { foods (e.g. } \\
\text { steamed rice, } \\
\text { cheddar } \\
\text { cheese, jam } \\
\text { doughnut). } \\
\text { Measure: } \\
\text { grams and } \\
\text { calories. }\end{array}$ & $\checkmark$ & $x$ & $\begin{array}{l}\text { Mood manipu } \\
\text { increase in neg } \\
\text { and no chang } \\
\text { condition show } \\
\text { and decrease in } \\
\text { No significant } 1 \\
\text { and no signif } \\
\text { differences in } \\
\text { restraint eater } \\
\text { intake for emo } \\
\text { eaters, but in tl } \\
\text { eaters consume } \\
\text { fatty foods thar }\end{array}$ \\
\hline $\begin{array}{l}\text { Royal \& } \\
\text { Kurtz (2010) }\end{array}$ & $\begin{array}{l}52 \text { female } \\
\text { students } \\
\text { Age: } 19.30 \\
\text { years }(1.11) \\
\text { BMI: } 21.63 \\
(2.65)\end{array}$ & $\begin{array}{l}\text { Stress } \\
\text { manipulation: } \\
\text { negative = } 10 \\
\text { unsolvable } \\
\text { anagrams and } \\
\text { neutral = } 10 \\
\text { solvable } \\
\text { anagrams. }\end{array}$ & $\begin{array}{l}\text { Mixed snacks: } \\
\text { M \& M's®, } \\
\text { Reese's } \\
\text { Pieces®, } \\
\text { cheese } \\
\text { crackers and } \\
\text { peanuts. } \\
\text { Measure: }\end{array}$ & $\checkmark$ & $\checkmark$ & $\begin{array}{l}\text { Mood manipu } \\
\text { anagrams cause } \\
\text { the solvable an } \\
\text { High stress con } \\
\text { than the low st } \\
\text { effect or inte } \\
\text { restraint was } \\
\text { overestimated }\end{array}$ \\
\hline
\end{tabular}




\begin{tabular}{|c|c|c|c|c|c|c|}
\hline & & $\begin{array}{l}\text { Measure: } \\
\text { PANAS }\end{array}$ & $\begin{array}{l}\text { pieces and } \\
\text { caloric value. }\end{array}$ & & & $\begin{array}{l}\text { high stress co } \\
\text { eaters overestir }\end{array}$ \\
\hline $\begin{array}{l}\text { Scattolon \& } \\
\text { Nicki (1995) }\end{array}$ & $\begin{array}{l}75 \text { females } \\
\text { High restraint } \\
\text { and worry }\end{array}$ & $\begin{array}{l}\text { Autobiographical } \\
\text { recall: } \\
\text { social/school } \\
\text { related, eating } \\
\text { related and non- } \\
\text { worry. } \\
\text { Measure: N/A }\end{array}$ & $\begin{array}{l}\text { Savoury } \\
\text { snacks: } \\
\text { regular, } \\
\text { barbeque and } \\
\text { salt and } \\
\text { vinegar potato } \\
\text { chips } \\
\text { Measure: } \\
\text { grams. }\end{array}$ & - & $\sqrt{ }$ & $\begin{array}{l}\text { No manipulatic } \\
\text { Significant effe } \\
\text { in the social/s } \\
\text { eating and non- }\end{array}$ \\
\hline $\begin{array}{l}\text { Schneider et } \\
\text { al. (2010) }\end{array}$ & $\begin{array}{l}37 \text { lean adults } \\
\text { Age: } 31.78 \\
\text { years }(10.59) \\
\text { BMI: } 22.79 \\
(1.72) \\
24 \text { obese adults } \\
\text { Age: } 38.96 \\
\text { years }(11.36) \\
\text { BMI: } 34.91 \\
(4.02)\end{array}$ & $\begin{array}{l}\text { Autobiographical } \\
\text { recall: anxious, } \\
\text { anger, and } \\
\text { neutral. Highest } \\
\text { rated memory } \\
\text { from a pre-study } \\
\text { interview. } \\
\text { Measure: } \\
\text { POMS. } \\
\text { Measure: POMS } \\
\text { anger and } \\
\text { anxiety } \\
\text { subscales }\end{array}$ & $\begin{array}{l}\text { Mixed snacks: } \\
6 \text { sweet and } \\
\text { savoury pre- } \\
\text { rated as highly } \\
\text { palatable, e.g. } \\
\text { peanut butter } \\
\text { cups, pretzels, } \\
\text { chips, cookies. } \\
\text { Measure: } \\
\text { caloric value. }\end{array}$ & $\checkmark$ & $\sqrt{ }$ & $\begin{array}{l}\text { Mood manip } \\
\text { anxiety and } \\
\text { increased follor } \\
\text { baseline. } \\
\text { Significantly g } \\
\text { higher in trait a } \\
\text { the neutral con } \\
\text { but not for lear } \\
\text { anxiety being } \mathrm{s} \\
\text { association bet } \\
\text { the anger condi }\end{array}$ \\
\hline $\begin{array}{l}\text { Schulz \& } \\
\text { Laessle } \\
(2012)\end{array}$ & $\begin{array}{l}35 \text { females with } \\
\text { BED } \\
\text { Age: } 32.4 \text { years } \\
\text { (8.0) } \\
\text { BMI: } 37.0(5.5)\end{array}$ & $\begin{array}{l}\text { Stress } \\
\text { manipulation: } \\
\text { negative = } \\
\text { TSST and } \\
\text { neutral = }\end{array}$ & $\begin{array}{l}\text { Sweet snack: } \\
\text { Chocolate } \\
\text { pudding. } \\
\text { Measure: } \\
\text { caloric value. }\end{array}$ & $v$ & $x$ & $\begin{array}{l}\text { Mood manipu } \\
\text { groups, the TS } \\
\text { stress. } \\
\text { No significant } \\
\text { between the tr }\end{array}$ \\
\hline
\end{tabular}


36 females

without BED

Age: 35.6 years

(8.4)

BMI: 36.9 (6.1)

\section{reading a}

newspaper.

Measure: VAS.

groups. Indivi initial eating $r$ control condit BED showed BED also shov intake in the compared to showed less de Mood manipu increase in sa Opposite found Relative to th eaters consun compared to $n$ reduced intake neutral film ir changes in rest

BFP/H: 96.8\%

Sproesser et 251 males and al. (2013) females Age: 24 years (6) negative $=$ hot buttered and salted Magnolias" and popcorn. BFP/H: $95.1 \%$ neutral $=\quad$ Measure: 19 unrestrained "G'day grams. eaters Australia." Age: 19.2 years Measure: VAS.

BMI: 23 (3)

Grouped into 45 hyper-phagics and 96 hypophagics. (additional group who

\section{Social Sweet snacks:}

exclusion: social three inclusion $=$ accepting feedback, social exclusion $=$ rejecting

feedback and neutral $=$ neutral feedback from a types/flavour of ice-cream.

Measure:

grams and caloric value.

Mood manipu
linear decreas
inclusion, neut
No significant
behaviour but
interaction was
condition, st
significantly m
the social in
phagics ate sig




\begin{tabular}{|c|c|c|c|c|c|c|}
\hline & $\begin{array}{l}\text { reported eating } \\
\text { to be unaffected } \\
\text { by stress) }\end{array}$ & $\begin{array}{l}\text { first impressions } \\
\text { questionnaire. } \\
\text { Measure: } \\
\text { authors own. }\end{array}$ & & & & $\begin{array}{l}\text { phagics. Simila } \\
\text { both groups in }\end{array}$ \\
\hline $\begin{array}{l}\text { Turner et al. } \\
\text { (2010) }\end{array}$ & $\begin{array}{l}106 \text { students } \\
\text { Age: } 23.46 \\
\text { years }(6.40) \\
\text { BMI: } 22.97 \\
(4.5) \\
\text { EE: TFEQ-R - } \\
1.88(.086)\end{array}$ & $\begin{array}{l}\text { Film excerpts; } \\
\text { positive = } \\
\text { "comedy } \\
\text { program" and } \\
\text { control } \\
\text { condition = } \\
\text { "science } \\
\text { documentary" } \\
\text { Measure: PA } \\
\text { from PANAS. }\end{array}$ & $\begin{array}{l}\text { Sweet snack: } \\
\text { Cookies } \\
\text { Measure: } \\
\text { Number of } \\
\text { cookies and } \\
\text { caloric value. }\end{array}$ & $\checkmark$ & $\checkmark$ & $\begin{array}{l}\text { Mood manipu } \\
\text { condition repor } \\
\text { control conditic } \\
\text { Control condit } \\
\text { more (on aver } \\
\text { Association b } \\
\text { calorie intake } \\
\text { uncontrolled } \\
\text { consumed less } \\
\text { condition. }\end{array}$ \\
\hline $\begin{array}{l}\text { Udo et al. } \\
\text { (2013) }\end{array}$ & $\begin{array}{l}15 \text { healthy } \\
\text { individuals }(\mathrm{F}= \\
46.7 \%) \\
\text { Age: } 31.7 \text { years } \\
(11.9) \\
\text { BMI: } 23.0 \\
(1.96) \\
15 \text { individuals } \\
\text { with obesity (F } \\
=40.0 \%) \\
\text { Age: } 41.9(11.6) \\
\text { BMI: } 35.1(3.7)\end{array}$ & $\begin{array}{l}\text { Guided imagery: } \\
\text { negative = } \\
\text { recent most } \\
\text { stressful event, } \\
\text { and positive = } \\
\text { positive event } \\
\text { that only } \\
\text { involved } \\
\text { themselves. } \\
\text { Measure: RDES }\end{array}$ & $\begin{array}{l}\text { Mixed snacks: } \\
3 \text { sweet and } 3 \\
\text { savoury } \\
\text { selected by } \\
\text { participants } \\
\text { e.g. potato } \\
\text { chips, popcorn, } \\
\text { peanuts, } \\
\text { cookies, } \\
\text { chocolate } \\
\text { pudding } \\
\text { Measure: } \\
\text { caloric value }\end{array}$ & $\checkmark$ & $\checkmark$ & $\begin{array}{l}\text { Mood manipu } \\
\text { emotion increa } \\
\text { decreased after } \\
\text { Individuals wi } \\
\text { more calories } i \\
\text { to the negativ } \\
\text { healthy individ } \\
\text { able to resist e } \\
\text { positive condit } \\
\text { increased after }\end{array}$ \\
\hline
\end{tabular}




\begin{tabular}{|c|c|c|c|c|c|c|}
\hline $\begin{array}{l}\text { van Strien \& } \\
\text { Ouwens } \\
(2007)\end{array}$ & $\begin{array}{l}86 \text { females } \\
\text { Age: } 21.1 \text { years } \\
(\mathrm{SD}=1.88) \\
\text { BMI: } 23.0 \\
(3.45)\end{array}$ & $\begin{array}{l}\text { Stress } \\
\text { manipulation: } \\
\text { high social } \\
\text { anxiety = word } \\
\text { fluency test, and } \\
\text { control } \\
\text { condition = } \\
\text { fabrics. } \\
\text { Measure: POMS }\end{array}$ & $\begin{array}{l}\text { Savoury snack: } \\
\text { TUC crackers } \\
\text { - natural, } \\
\text { paprika and } \\
\text { olive/rosemary } \\
\text { Measure: } \\
\text { grams }\end{array}$ & $\checkmark$ & $x$ & $\begin{array}{l}\text { Mood manipul } \\
\text { increase in tens } \\
\text { significant decr } \\
\text { No significant } \\
\text { intake betwe } \\
\text { association b } \\
\text { DIF/DDF or in } \\
\text { DIF/DDF inte } \\
\text { levels of DIF } \\
\text { consumed less } \\
\text { Condition } x \text { im }\end{array}$ \\
\hline \multirow[t]{2}{*}{$\begin{array}{l}\text { van Strien et } \\
\text { al. (2012) }\end{array}$} & $\begin{array}{l}\text { Study } 1 \\
124 \text { female } \\
\text { students } \\
\text { Age: } 21.8 \text { years } \\
(3.6) \\
\text { BMI: } 23.3(3.7) \\
\text { EE: DEBQ split } \\
\text { into low }=1.82 \\
\text { and high }=3.25\end{array}$ & $\begin{array}{l}\text { Film excerpts: } \\
\text { sad = "breaking } \\
\text { the waves" and } \\
\text { neutral = } \\
\text { "travelling } \\
\text { birds" } \\
\text { Measure: VAS }\end{array}$ & $\begin{array}{l}\text { Mixed snacks: } \\
\text { crisps and M } \\
\& \text { M's® } \\
\text { Measure: } \\
\text { caloric value }\end{array}$ & $\checkmark$ & $*$ & $\begin{array}{l}\text { Mood manipu } \\
\text { condition repor } \\
\text { condition. } \\
\text { Self-reported } \\
\text { moderated the } \\
\text { snack food. Lo } \\
\text { during the sac } \\
\text { movie, wherea } \\
\text { slightly more d }\end{array}$ \\
\hline & $\begin{array}{l}\text { Study } 2 \\
47 \text { female } \\
\text { students } \\
\text { Age: } 19 \text { years } \\
(18-27) \\
\text { BMI: } 21.27 \\
(2.66)\end{array}$ & $\begin{array}{l}\text { Stress } \\
\text { manipulation: } \\
\text { negative = } \\
\text { TSST and } \\
\text { neutral = fabrics } \\
\text { Measure: } \\
\text { PANAS }\end{array}$ & $\begin{array}{l}\text { Sweet snacks: } \\
\text { white grapes, } \\
\text { carrot, M \& } \\
\text { M's }{ }^{\circledR} \text { and } \\
\text { butter cake. } \\
\text { Measure: } \\
\text { caloric value }\end{array}$ & $\checkmark$ & $*$ & $\begin{array}{l}\text { Mood manip } \\
\text { condition rep } \\
\text { irritated, shame } \\
\text { Emotional eati } \\
\text { low emotional } \\
\text { task than the c } \\
\text { consumed mo } \\
\text { emotional eater }\end{array}$ \\
\hline
\end{tabular}


EE: DEBQ split

into low $=1.82$

and high $=3.25$

van Strien et

al. (2013)

29 low emotional eaters
Age: 25.4 years

(9.2)

BMI: 21.6 (3.0)

EE: DEBQ <

1.8

31 high

emotional eaters

Age: 22.6 years

(3.3)

BMI: 22.5 (2.8)

EE: DEBQ >

2.6

van Strien et

al. (2014)

25 low EE

females (DEBQ

$<1.82$ )

BMI: 20.30

(1.39)

29 high EE

females (DEBQ

$>3.25$ )

BMI: 21.92

(2.80) after both conc

eaters reported

Virtual reality: Mixed snacks:

Urban park apple, banana,

setting; joy $=\quad$ salty peanuts,

"Singing in the sweet peanuts,

Rain" and

sadness = "The

chips, jellies,

cereal bar,

chocolate, rice

Champ".

diet bar and

rosquilleta.

Measure:

caloric value
Mood manipu. increase in $\mathrm{V}$. sadness conditi scores for the $\mathrm{jc}$ No significant 1 a borderline si condition. $\mathrm{Gr}$ condition com emotional eate both conditions consumed sign Emotional eati sweet food inta

Mood manipu peak in mean between stress the stress task. Mean food int across condit positively relat Emotional eati and snack in reduction in condition. 
Overall age:

21.17 years

(2.32)

\begin{tabular}{|c|c|c|c|c|c|c|}
\hline $\begin{array}{l}\text { Wallis \& } \\
\text { Hetherington } \\
(2004)\end{array}$ & $\begin{array}{l}38 \text { females } \\
\text { Age: average } \\
\text { range }=21.7- \\
27.1 \text { years } \\
\text { BMI: average } \\
\text { range }=23.3- \\
24.8 \\
\text { EE: average } \\
\text { range }(\mathrm{DEBQ})= \\
2.0-3.7\end{array}$ & $\begin{array}{l}\text { Stroop task: } \\
\text { negative = ego- } \\
\text { threatening, } \\
\text { neutral = } \\
\text { congruent } \\
\text { colour/words, } \\
\text { and cognitive } \\
\text { load = } \\
\text { incongruent } \\
\text { colour/word. } \\
\text { Measure: } \\
\text { PANAS }\end{array}$ & $\begin{array}{l}\text { Sweet snack: } \\
\text { Cadbury’s }{ }^{\circledR} \\
\text { Chocolate } \\
\text { buttons. } \\
\text { Measure: } \\
\text { grams }\end{array}$ & $\checkmark$ & $\checkmark$ & $\begin{array}{l}\text { Mood manipu } \\
\text { decrease in pos } \\
\text { condition. Ne } \\
\text { significantly le } \\
\text { incongruent co } \\
\text { Significantly } \\
\text { consumed in } \\
\text { conditions than } \\
\text { group consume } \\
\text { whereas HRLE } \\
\text { the ego-threat } \\
\text { HRHE only c } \\
\text { condition. }\end{array}$ \\
\hline $\begin{array}{l}\text { Wallis \& } \\
\text { Hetherington } \\
\text { (2009) }\end{array}$ & $\begin{array}{l}26 \text { females } \\
\text { Age: average } \\
\text { range }=23.7- \\
30.8 \text { years } \\
\text { BMI: average } \\
\text { range = } 22.4- \\
26.1 \\
\text { EE: DEBQ } \\
\text { median split } \\
(2.5)\end{array}$ & $\begin{array}{l}\text { Stroop task: } \\
\text { negative = ego- } \\
\text { threatening, and } \\
\text { control = neutral } \\
\text { words. } \\
\text { Measure: VAS } \\
\text { and PANAS }\end{array}$ & $\begin{array}{l}\text { Sweet snack: } \\
\text { dried fruit mix } \\
\text { and } \\
\text { Cadbury’s }{ }^{\circledR} \\
\text { Chocolate } \\
\text { buttons. } \\
\text { Measure: } \\
\text { grams }\end{array}$ & $\checkmark$ & $x$ & $\begin{array}{l}\text { Mood manipu } \\
\text { increase in anx } \\
\text { after the ego-th } \\
\text { control task. } \\
\text { Similar amoun } \\
\text { conditions. } \\
\text { significantly m } \\
\text { compared to th } \\
\text { for restrained } \\
\text { across high and }\end{array}$ \\
\hline
\end{tabular}




\begin{tabular}{|c|c|c|c|c|c|c|}
\hline $\begin{array}{l}\text { Werthmann } \\
\text { et al. (2014) }\end{array}$ & $\begin{array}{l}85 \text { female } \\
\text { students } \\
\text { Age: average } \\
\text { range }=20.48- \\
20.81 \\
\text { BMI: average } \\
\text { range = 22.13- } \\
22.4 \\
\text { EE: DEBQ } \\
\text { average range = } \\
2.58-2.7\end{array}$ & $\begin{array}{l}\text { Mixed: negative } \\
=\text { "Adagio for } \\
\text { strings" and } \\
\text { recall a negative } \\
\text { event, and } \\
\text { neutral = } \\
\text { "Dancing with } \\
\text { the sun" and } \\
\text { recall of recent } \\
\text { course study. } \\
\text { Measure: VAS }\end{array}$ & $\begin{array}{l}\text { Mixed snacks: } \\
\text { grapes, } \\
\text { cucumber, } \\
\text { chocolate and } \\
\text { chips. } \\
\text { Measure: } \\
\text { grams }\end{array}$ & $\checkmark$ & $*$ & $\begin{array}{l}\text { Mood manipu } \\
\text { decrease in mo } \\
\text { change in the } \mathrm{n} \\
\text { Significantly } \mathrm{h} \\
\text { the in the ne } \\
\text { condition not } \\
\text { effect of sel } \\
\text { condition } \mathrm{x} \text { el } \\
\text { reported emotic } \\
\text { food intake dur } \\
\text { it associated wi }\end{array}$ \\
\hline $\begin{array}{l}\text { Yeomans \& } \\
\text { Coughlan } \\
(2009)\end{array}$ & $\begin{array}{l}96 \text { females } \\
\text { Age: average } \\
\text { range }=20-25 \\
\text { years. } \\
\text { BMI: range = } \\
20.6-23.7\end{array}$ & $\begin{array}{l}\text { Film excerpts: } \\
\text { negative = "The } \\
\text { Shinning", } \\
\text { positive = } \\
\text { "Friends" and } \\
\text { neutral = } \\
\text { "Exmoor video." } \\
\text { Measure: POMS }\end{array}$ & $\begin{array}{l}\text { Mixed snacks: } \\
\text { salted Popz® } \\
\text { popcorn and } \\
\text { Sainsbury’s } \\
\text { Taste the } \\
\text { Difference® } \\
\text { raisins. } \\
\text { Measure: } \\
\text { grams and } \\
\text { caloric value. }\end{array}$ & $\sqrt{ }$ & $*$ & $\begin{array}{l}\text { Mood manipul } \\
\text { negative condit } \\
\text { former induced } \\
\text { mood states and } \\
\text { states. } \\
\text { The HRHD gro } \\
\text { the negative co } \\
\text { conditions. } \\
\text { significantly m } \\
\text { marginally mo } \\
\text { negative cond } \\
\text { differences in } \\
\text { groups. }\end{array}$ \\
\hline
\end{tabular}

$\checkmark=$ significant difference, $X=$ no significant difference, $*=$ no main effect of condition reported, AN $=$ Anc Disorder, $\mathrm{BMI}=$ Body Mass Index, BN = Bulimia Nervosa, DDF $=$ difficulty describing feelings, $\mathrm{EE}=\mathrm{em}$ high restraint, low emotional, LRHD = low restraint, high disinhibition, LRLE = low restraint, low em disinhibition, HRHD = high restraint, high disinhibition, HRLD = high restraint, low disinhibition, HS = hu N/A = not applicable, POMS $=$ profile of mood States, $\mathrm{RDES}=$ revised differential emotion scale, $\mathrm{TSST}=\mathrm{T}$ 


\subsection{Study Four: Aims}

The primary aim of Study Four was to explore the effect of mood manipulation on food intake. To determine whether the mood induction paradigm successfully elicited changes in food intake, the first step was to successfully induce a change in emotional state. No significant changes in POMS overall mood were expected from pre- to post-mood manipulation $\left(T_{1}\right.$ to $\left.T_{2}\right)$ in the control condition. However, for participants in the positive condition, elatedness scores were predicted to increase following manipulation of mood, with the opposite predicted to occur in the negative condition. For the emotionally confusing condition, a decrease in participant's selfreported levels of clear-headedness and composure were predicted, as well as an increase in 'mixed emotions' (SME) ratings from $T_{1}$ to $T_{2}$. These predictions were in line with findings from Study Three.

The second step involved testing differences in food intake following the experimental conditions compared to the control condition. Significantly greater food intake was predicted in the negative and emotional confusing conditions, with the latter predicted to consume the most. Additional analyses were conducted to explore the influence of alexithymia and emotional eating on food intake following the mood manipulation. It was predicted that participants with alexithymia and self-reported emotional eaters would consume the greatest amounts of food following the negative and emotionally confusing conditions.

The third step aimed to predict emotional eating and to advance the model proposed in Chapter Two. Firstly, it aimed to further test the model of self-reported emotional eating. In line with the findings from Study One and Two, it was predicted that alexithymia would indirectly predict emotional eating through negative affect and 
negative urgency. Secondly, it aimed to advance the model to test its ability to predict actual food intake. A significant indirect effect via negative affect and negative urgency was expected.

\subsection{Study Four: Methods}

\subsubsection{Participants}

One hundred and thirty-seven participants took part in the study. However, some participants were subsequently removed for the following reasons: (a) did not take part in both sessions ( $n=5)$; (b) consumed all of the food presented in both study sessions $(n=8)$; (c) reporting extreme scores on the BDI and/or BAI $(n=2)$, and (d) technical issues that affected the accuracy of data recorded $(n=1)$. Of the remaining 121 participants, $86(71.1 \%)$ were female and mean age was 24.21 years old $(S D=$ 6.23 years, range $=18.33-53.36$ years $)$. Two participants incorrectly inputted their date of birth, and therefore, age could not be calculated and were marked as missing values. The majority of participants identified themselves as single $(n=106 ; 87.6 \%)$, with a further eight $(6.6 \%)$ married, five $(4.1 \%)$ co-habiting, and one $(.8 \%)$ divorced. Participants identified their ethnicity as White British $(n=97 ; 80.2 \%)$, Asian $(n=9$, $6.6 \%)$, Black $(n=4,3.3 \%)$, Chinese $(n=7,5.8 \%)$, Mixed $(n=2 ; 1.7 \%)$, other $(n=1$, $.8 \%)$, or preferred not to say $(n=1, .8 \%)$. Most participants were either in full/part time education $(n=97 ; 80.2 \%)$ or in full-time $(n=13 ; 10.7 \%)$ or part-time $(n=7$, $5.8 \%)$ employment. Two participants $(1.7 \%)$ were unemployed. BMI scores ranged from $17.61-48.93(M=25.3, S D=4.83)$ and mean percentage body fat was $27.41(S D$ $=8.92 ;$ range $=10.80-59.20)$. Participants were systematically allocated to either a 
positive $(n=39)$, negative $(n=41)$ or emotionally confusing $(n=41)$ group. As with Study 3, participants were randomly allocated to reduce bias and potential confounding variables (Schulz \& Grimes, 2002). In order to keep the size of groups similar, random allocation in blocks was conducted. A random sequence of groups (14) was generated in blocks of four and repeated until a sufficient sample size was reached.

An a priori power analysis ( $G^{*}$ Power; Faul et al., 2007) indicated that a minimum sample size of 120 should be used (effect size $=.25$ - medium; power $=.8$ ). Therefore, the current study was sufficiently powered $(N=121)$.

\subsubsection{Measures}

\subsubsection{Demographics}

Participants were asked to provide their date of birth, sex, marital status, employment status, ethnicity, and information to determine the number of years they had spent in formal education.

\subsubsection{Profile of Mood States}

(POMS; Lorr \& McNair, 1982; McNair et al., 1971, 1992). As fully described in Chapter Three (section 3.4.2.2.). The POMS was administered at six time points and the internal consistency of the overall POMS score was excellent, $\alpha=.95-.97$.

\subsubsection{Subjective measure of Mixed Emotions}

(MEQ; Berrios et al., 2013; Berrios et al., 2015b, 2017). As fully described in Chapter Three (section 3.4.2.3.), the Cronbach's alphas for the current study across the six time points were $\alpha=.88-.93$. 


\subsubsection{Toronto Alexithymia Scale}

(TAS-20; Bagby, Parker, et al., 1994; Bagby, Taylor, et al., 1994). As fully described in Chapter Two (section 2.3.2.3.). Cronbach's alphas for the current study were: DIF $\alpha=.8$; DDF $\alpha=.79$; EOT $\alpha=.65$, and total TAS-20 scores $\alpha=.83$.

\subsubsection{Beck Depression Inventory-II}

(BDI; Beck et al., 1961; Beck et al., 1996;Beck, Steer, et al., 1988). The BDI evidenced good levels of internal consistency $(\alpha=.84)$ and is described in Chapter Two (section 2.3.2.5.).

\subsubsection{Beck Anxiety Inventory}

(BAI; Beck, Epstein, et al., 1988; Beck \& Steer, 1990). The BAI evidenced excellent levels of internal reliability $(\alpha=.9)$ and a full description of the measure can be found in Chapter Two (section 2.3.2.6.).

\subsubsection{UPPS-P}

(Cyders et al., 2007; Whiteside \& Lynam, 2001). The UPPS-P is described in Chapter Two (section 2.3.2.4.). The UPPS-P was administered in full but only responses to the negative urgency (NU) subscale are reported here $(\alpha=.87)$.

\subsubsection{Emotional Eating Scale}

(EES; Arnow, Kenardy, \& Agras, 1995). A full description of the EES is presented in Chapter Two (section 2.3.2.7.) and the scale evidenced acceptable to high levels of internal consistency in the current study (ang/frus $\alpha=.80 ;$ anx $\alpha=.75$; $\operatorname{dep} \alpha$ $=.71 ;$ total EES scores $\alpha=.88$ ).

\subsubsection{Three Factor Eating Questioned - revised}

(TFEQ; Karlsson, Persson, Sjöström, \& Sullivan, 2000). The TFEQ is fully described in Chapter Two (section 2.3.2.8.). Acceptable levels of internal consistency 
were found in the current study (emotional eating $\alpha=.71$, uncontrolled eating $\alpha=.75$, and cognitive restraint $\alpha=.77$ ).

\subsubsection{Memory Characteristic Questionnaire}

(MCQ; Johnson et al., 1988). As fully described in Chapter Three (section 3.4.2.7.) was also given to participants but only as part of the cover story.

\subsubsection{Materials}

\subsubsection{Mood Induction}

The novel mood induction paradigm developed in Chapter Three was used with some slight modifications. First, and as discussed in Chapter Three (see section 3.6), the time allocated for participants to recall and write about their chosen memory was extended from seven to ten minutes. Second, clarifying statements (e.g. "by this I mean an occasion where you have felt particularly elated/upset") were added to the written instructions for the positive and negative conditions. These changes were made to further aid participants understanding of the task and to ensure consistency in written instructions across conditions. Finally, additional prompts to guide participants engagement with their identified autobiographical memory were added (e.g. "think about the people involved, what sounds were present, and the thoughts you had at the time”). See Appendix S for full details.

\subsubsection{Bogus Taste Test}

Participants were presented with three savoury and three sweet snacks in silicon cupcake cases; eight Galaxy® Minstrels® (Mars Incorporated, Virginia, US), eight Pringles ${ }^{\circledR}$ Original (Kellogg's, Michigan, US; 8 pringles), six Bitsa Wipsa ${ }^{\circledR}$ (Cadbury, (Mondelez International), London, UK), roughly 8 Haribo® Starmix ${ }^{\circledR}$ 
sweets (Haribo, Bonn, Germany), eight Jacob's ${ }^{\circledR}$ Mini Cheddars ${ }^{\circledR}$ (McVitie's, Edinburgh, UK) and roughly 2-3g (fill the cupcake cases) Tesco Salted Popcorn (Tesco, Hertfordshire, UK; see Appendix T for further details). These snacks were chosen based on previous work within the Swansea Nutrition, Appetite and Cognition (SNAC) group at Swansea University (Price et al., 2016). Participants were instructed to "...try all the snacks at least once but feel free to eat as much as you wish as any left overs have to be disposed of for health and safety reasons. There is water provided for you. Please take your time when completing the questions". To accompany the snacks and to corroborate the cover story for the study, participants were asked to complete a taste perception questionnaire, including items such as "How much do you like this snack? How strong is the flavour of this snack?" Responses were recorded on an 11-point scale from "0" (e.g. "not very much/not very strong") to "10" (e.g. "extremely/very strong;" see Appendix U for full details). Snacks were weighed before and after the bogus taste test to calculate how much food participants had consumed (using Sartorius CP4201 scales). All participants received the same amount of each snack and the maximum possible total weight consumption ranged from $98-104.4 \mathrm{~g}$. Food weight was used as the dependent variable instead of calories/energy intake as the former has been found to be a stronger determinant of food intake (WesterterpPlantenga, 2004).

\subsubsection{Design}

The current study was a $2 \times 2$ repeated measures design where every participant completed one experimental condition and the control condition. Consequently, every participant acted as their own control in the analysis, a method used in previous food 
intake studies (Appelhans et al., 2011). The three experimental conditions were: positive, negative and emotional confusion. Overall food intake was measured in grams.

\subsubsection{Procedure}

Ethical approval was obtained from the Department of Psychology Ethics Committee, College of Human and Health Sciences, Swansea University. Participants were recruited through Swansea University's Student Newsletter and participants took part in exchange for a monetary reward (£12 cash or $£ 15$ high street vouchers) or subject pool credit. Participants were recruited under the pretence that they were completing a study on the effects of memory recall on taste perception. Specifically, the study was advertised as "An investigation into the effect of memory recall on taste perception". Participants were told the purpose of the study was to test the effect of recalling a memory of different emotional valence (either positive, negative or emotional confusion) on our taste perception of food.

\subsubsection{Testing session 1}

All participants were asked not to eat or drink anything except water for two hours before the study. After consent was provided, participants completed the demographic information and the initial POMS, SME, BDI and BAI via a digitalised questionnaire using Survey Monkey (Palo Alto, California, USA). When directed on screen, participants called the experimenter to begin the mood induction task and received the instructions for the study verbally and in written format (see Appendix S). Participants were presented with the instructions for either a control, positive, negative or emotionally confusing condition depending on their allocation. 
Participants' understanding of the task was checked prior to commencing the moodmanipulation phase. Participants were then given 10 minutes to recall and write about their chosen memory before the experimenter re-entered the room. If participants finished early, they were instructed to let the experimenter know and if this occurred, participants were then instructed to double check they had sufficiently recalled their memory in enough detail before moving on. Participants were then asked to complete the POMS and SME at Time 2. After completion, participants were presented with the taste perception task. As described in section 4.3.3.2. participants completed the taste test in their own time. Participants were left unsupervised for an unlimited amount of time and were asked to notify the experimenter when they were done. The tray containing the food items was then removed from the participants, where they proceeded to complete the Time 3 POMS and SME, as well as the MCQ. Participants were thanked for their time and a time for testing session 2 was confirmed (see Appendix V for flow diagram).

\subsubsection{Testing session 2}

Participants were informed that the second testing session would be similar to session 1 and were asked if they had any questions before proceeding. Participants were instructed to work through the POMS and SME (Time 1) and to notify the experimenter when they had finished. Participants were then presented with their second condition (either the control or an experimental condition). Following this, participants completed the POMS and SME (Time 2) again before completing the taste perception task. They then completed the final POMS and SME (Time 3) as well as the TAS-20, EES and TFEQ. Height, weight, waist circumference and body fat percentage were taken for each participant before they were fully debriefed about the

true aims of the study. To check that the cover story had worked, participants were 
asked at the end of the study whether they had suspected any additional aims to the study other than those reported at the start.

\subsubsection{Statistical Analysis}

\subsubsection{Manipulation check}

To check that the mood induction was successful, difference scores from the control and experimental conditions were tested using a repeated measures ANOVA. Specifically, baseline (Time $1 ; T_{1}$ ) mood scores were subtracted from post-mood (Time $2 ; T_{2}$ ) scores to produce a difference score for the control condition and the experimental condition. Thus, a positive difference score indicates an increase in mood, whereas a negative score indicates a decrease in mood. SME, total POMS mood, elatedness, clear-headedness and composure subscales were all examined in the current analysis. When homogeneity of variance was violated, Brown-Forsythe F-ratio was reported and Games-Howell post-hoc analyses were conducted.

\subsubsection{Food intake}

Total food intake was calculated for the control and experimental conditions by subtracting the amount of food left behind from total start weight in grams. A oneway ANOVA was conducted on food intake difference scores, and in line with Turner et al. (2010), BMI, hunger, and restraint scores were controlled for in the analysis. Post-hoc Bonferroni pairwise comparisons were conducted to explore specific differences across the groups.

To explore whether alexithymia could predict difference in food intake scores, a hierarchical multiple regression was conducted. Difference in food intake scores 
were entered as the dependent variable with dummy coded group variables entered into step 1 and total TAS-20 scores entered in step 2.

The influence of emotional eating was also explored by grouping participants into high $(n=49,44.1 \%)$ and low $(n=62,55.9 \%)$ emotional eaters based on a median split of the EES. A two-way ANOVA was conducted with difference in intake score, condition and emotional eating. BMI, hunger, restraint and alexithymia scores were controlled for in the analysis. This was to control for the possible effects of the personality traits and to ensure any significant differences found were attributable to self-reported emotional eating only.

\subsubsection{Serial mediation model}

A series of serial mediation models were conducted to analyse the relationship between alexithymia and emotional eating. The first models entered total alexithymia scores at $X$ and the difference in food intake score at $Y$. Negative affect (BDI and BAI entered in separate models) and negative urgency were entered as mediators at $M_{1}$ and $M_{2}$ respectively. The second models entered self-reported emotional eating (as measured by EES and TFEQ) at $Y$. Table 14 outlines each model. 
Table 14. Serial multivariate mediation models analysed in Study Four.

\begin{tabular}{ll}
\hline Model & Pathway \\
\hline 1 & TAS-20 $\rightarrow$ BDI $\rightarrow$ NU $\rightarrow$ DIFF. EAT \\
2 & TAS-20 $\rightarrow$ BAI $\rightarrow$ NU $\rightarrow$ DIFF.EAT \\
3 & TAS-20 $\rightarrow$ BDI $\rightarrow$ NU $\rightarrow$ TFEQ-EE \\
4 & TAS-20 $\rightarrow$ BDI $\rightarrow$ NU $\rightarrow$ EES \\
5 & TAS-20 $\rightarrow$ BAI $\rightarrow$ NU $\rightarrow$ TFEQ-EE \\
6 & TAS-20 $\rightarrow$ BAI $\rightarrow$ NU $\rightarrow$ EES \\
\hline $\begin{array}{l}\text { BAI = Beck anxiety inventory, BDI }=\text { Beck depression inventory, DIFF.EAT }= \\
\text { difference in food intake, EES }=\text { emotional eating scale, TFEQ-EE = three factor eating } \\
\text { questionnaire emotional eating subscale, NU = negative urgency, TAS-20 = Toronto } \\
\text { alexithymia scale total score. }\end{array}$
\end{tabular}

\subsection{Study Four: Results}

\subsubsection{Descriptive Statistics}

In line with de Boer and colleagues (2015), inferential statistics were not performed to detect differences in demographics across the groups. Therefore, total and group means for all demographic and personality data is presented in Table 15.

\subsubsection{Manipulation Check}

\subsubsection{Control condition}

No significant difference in overall POMS, $F(2,118)=.22, p=.805$, or SME scores, $F(2,118)=.07, p=.937$, were found across groups. 


\subsubsection{Experimental condition}

A one-way ANOVA was conducted to test for significant differences between $T_{1}$ and $T_{2}$ for the total POMS score across groups for the experimental condition. As the assumption of homogeneity of variance was violated, the Brown-Forsythe $F$-ratio was used. There was a significant difference across groups, $F(2,96.08)=24.11, p<$ .001 , with post-hoc pairwise comparisons (Games-Howell) revealing that the positive group $(M=9.38, S D=14.61)$ reported a significantly higher overall POMS score than the negative $(M=-21.29, S D=22.16, p<.001)$ and emotionally confusing $(M=-$ 21.22, $S D=29.1, p<.001$, see Figure 12) groups.

A one-way ANOVA was also conducted to test for significant differences between $T_{1}$ and $T_{2}$ for SME total scores across groups for the experimental condition. A significant group difference emerged, $F(2,118)=6.23, p=.003$, with post-hoc pairwise comparisons (Bonferroni) revealing that the positive group $(M=.04, S D=$ .74) reported significantly lower SME scores than the negative $(M=.7, S D=.7, p=$ $.002)$ but not the emotionally confusing $(M=.43, S D=1.01, p=.123)$ group. 
Table 15. Total and group means, standard deviations and ranges for each study variable in Study Four.

\begin{tabular}{lcccccc}
\hline & \multicolumn{2}{c}{ Total } & \multicolumn{2}{c}{ Positive } & \multicolumn{2}{c}{ Negative } \\
\hline Measure & Mean $(S D)$ & Range & Mean (SD) & Range & Mean (SD) & Range \\
\hline DIF & $15.8(5.08)$ & $7.00-32.00$ & $16.87(5.14)$ & $9-32$ & $14.15(4.78)$ & $7-25$ \\
DDF & $14.72(4.46)$ & $5.00-25.00$ & $14.67(4.29)$ & $8-25$ & $14.76(4.01)$ & $5-22$ \\
EOT & $19.47(4.64)$ & $10.00-31.00$ & $20.44(4.45)$ & $10-31$ & $18.44(4.39)$ & $11-27$ \\
TAS-20 & $49.99(10.72)$ & $24.00-83.00$ & $51.97(10.69)$ & $34-83$ & $47.34(8.97)$ & $24-66$ \\
NU & $29.17(6.9)$ & $13.00-47.00$ & $30.26(6.46)$ & $19-43$ & $27.34(6.64)$ & $17-47$ \\
BDI & $8.61(6.38)$ & $.00-35.00$ & $9.08(6.06)$ & $0-26$ & $7.54(6.52)$ & $0-26$ \\
BAI & $8.33(7.89)$ & $.00-36.00$ & $9.26(8.83)$ & $1-36$ & $6.22(6.05)$ & $0-24$ \\
Ang/Frus & $21.38(6.7)$ & $11.00-39.00$ & $21.38(6.53)$ & $11-35$ & $20.98(6.79)$ & $11-34$ \\
Anx & $18.13(5.62)$ & $9.00-36.00$ & $18.21(6.11)$ & $9-37$ & $17.12(5.37)$ & $9-32$ \\
Dep & $14.82(5.62)$ & $5.00-25.00$ & $14.69(3.15)$ & $6-23$ & $14.73(4.25)$ & $6-25$ \\
EES & $54.33(13.99)$ & $25.00-95.00$ & $54.28(13.6)$ & $27-95$ & $52.83(14.64)$ & $28-91$ \\
TFEQ-CR & $45.45(14.25)$ & $14.29-78.57$ & $43.96(14.5)$ & $21.43-78$. & $45.12(14.85)$ & $17.86-78$. \\
TFEQ-UE & $66.69(13.03)$ & $36.11-105.56$ & $67.88(14.78)$ & $44.44-105.56$ & $64.63(12.04)$ & $41.67-94$. \\
TFEQ-EE & $57.78(18.22)$ & $25.00-100.00$ & $58.55(18.58)$ & $25-91.67$ & $54.67(19.01)$ & $25-91.6$ \\
\hline Ang/Fr $=$ & & & &
\end{tabular}

Ang/Frus = anger/frustration EES subscale, Anx = anxiety EES subscale, BAI = Beck anxiety inventory, BDI difficulty identifying feelings, DDF = difficulty describing feelings, Dep = depression EES subscale, EOT = emotional eating scale total score, $\mathrm{NU}=$ negative urgency, $\mathrm{TAS}-20=$ total Toronto alexithymia scale, $\mathrm{TF}$ subscale, TFEQ-EE = emotional eating TFEQ subscale, TFEQ-UE = uncontrolled eating TFEQ subscale. 


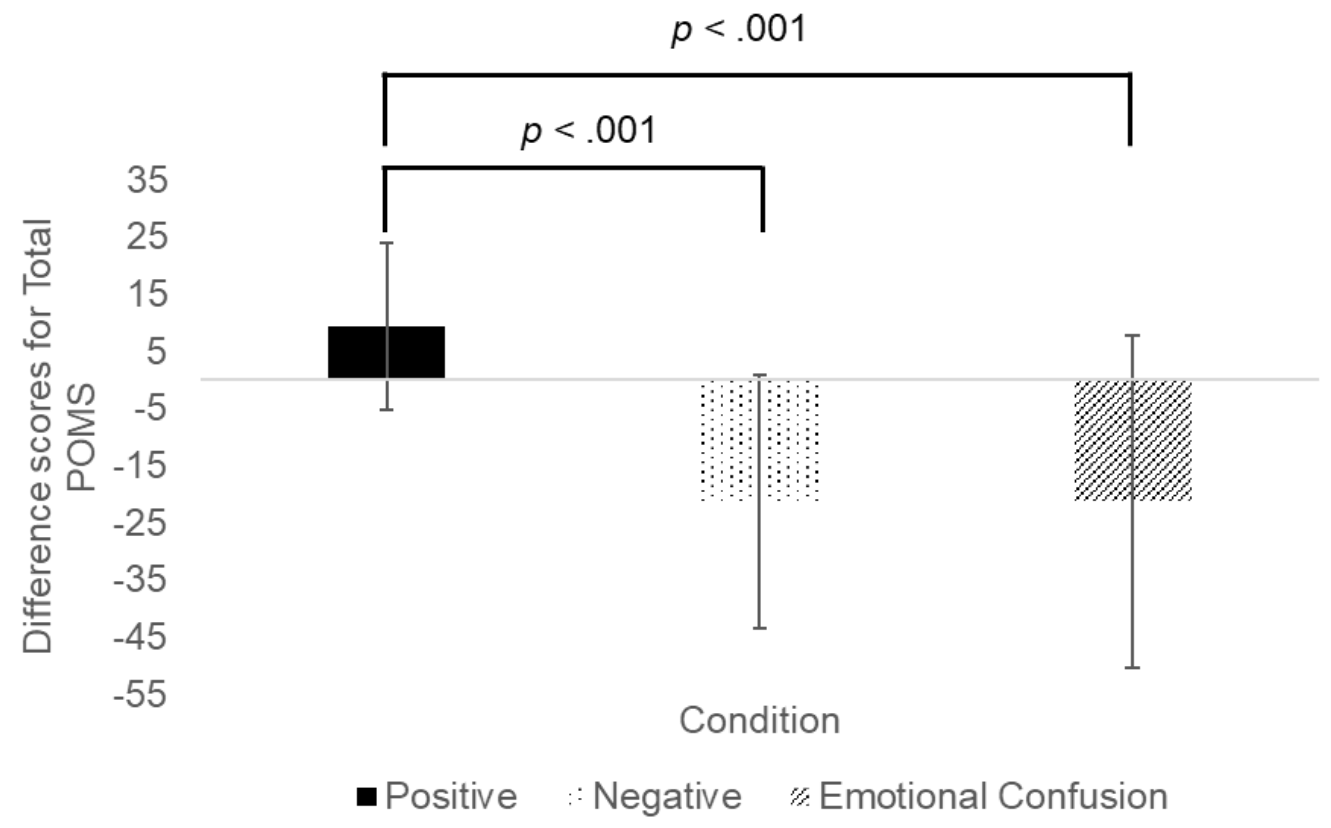

Figure 12. Differences in total POMS score baseline $\left(T_{1}\right)$ to post-mood $\left(T_{2}\right)$ for the positive, negative and emotional confusion groups. Error bars represent SD.

A series of one-way ANOVAs were also conducted to test for POMS subscale differences between $T_{1}$ and $T_{2}$ in the experimental condition. Elatedness scores were found to significantly differ across groups, $F(2,100.14)=40.77, p<.001$ (BrownForsythe $F$-ratio), with significantly higher scores in the positive $(M=2.08, S D=3.26)$ compared to negative $(M=-7.51, S D=5.05, p<.001)$ and emotionally confusing $(M$ $=-.5 .2, S D=6.12, p<.001)$ groups (Games-Howell). Clear-headedness scores also significantly differed across groups, $F(2,118)=11.75, p<.001$, with significantly higher scores in the positive $(M=1.49, S D=4.16)$ than negative $(M=-3.27, S D=$ $5.02, p<.001)$ and emotionally confusing $(M=-3.34, S D=5.88, p<.001)$ groups. Finally, composure subscale scores also differed significantly across groups, $F(2,118)$ $=8.65, p<.001$, with higher composure scores in the positive $(M=.56, S D=4.06)$ compared to negative $(M=-2.8, S D=4.97, p=.012)$ and emotionally confusing $(M=$ 
$-4.05, S D=6.07, p<.001$ ) groups (Bonferroni). For all three POMS subscale scores of interest, no significant differences were observed between the negative and emotionally confusing groups.

To conclude, the above findings suggest that mood manipulation was successful in differentiating the positive group from both the negative and emotionally confusing groups. However, post-hoc analyses found no significant differences between the negative and emotionally confusing groups on the POMS subscales. Therefore, and in contrast to the pilot data reported in Chapter Three, the emotionally confusing manipulation did not have the desired effect (e.g. significantly less composure and clear-headedness in the emotionally confusing versus negative group).

\subsubsection{Order Effects}

Although counterbalancing procedures were used, a one-way ANOVA was conducted to test for the possibility of order effects on the total POMS difference scores for the experimental condition (e.g. did the participants in positive group respond the same whether they had the control or experimental condition first). Participants were assigned to one of six groups depending on the order in which they completed the conditions $(1=$ control-positive, $2=$ control - negative, $3=$ controlemotional confusion, $4=$ positive-control, $5=$ negative-control, $6=$ emotional confusion-control). The overall ANOVA was significant, $F(5,115)=8.49, p<.001$. To ensure there were no significant differences in corresponding groups (e.g. group 1control-positive versus group 4-positive-control), pairwise comparisons (Bonferroni) were performed. No significant differences were found for corresponding groups, 
indicating that there were no order effects and the counterbalancing procedures were effective.

\subsubsection{Food Intake}

No participants identified that food intake was being measured in the study when asked if they thought there were any additional aims. This indicated that the cover story was successful and that all food intake data could be included in the analysis. Descriptive statistics for food intake across conditions can be found in Table 16.

Table 16. Mean (SD) food intake in grams for the control and experimental conditions across positive, negative and emotionally confusing groups.

\begin{tabular}{lccc}
\hline Condition & \multicolumn{3}{c}{ Mean food intake in grams $(S D)$} \\
\cline { 2 - 4 } Control & Positive & Negative & Emotional Confusion \\
\cline { 2 - 4 } Experimental & $50.67(21.14)$ & $55.98(26.09)$ & $64.7(30.86)$ \\
& $55.18(20.24)$ & $58.97(24.36)$ & $61.25(20.05)$ \\
\hline
\end{tabular}

A one-way ANOVA was conducted to test for significant differences in food intake (difference scores between control and experimental condition) across groups, controlling for cognitive restraint, hunger and BMI scores. Food intake did not significantly differ across groups, $F(2,118)=2.12, p=.125$, see Figure 13 . 

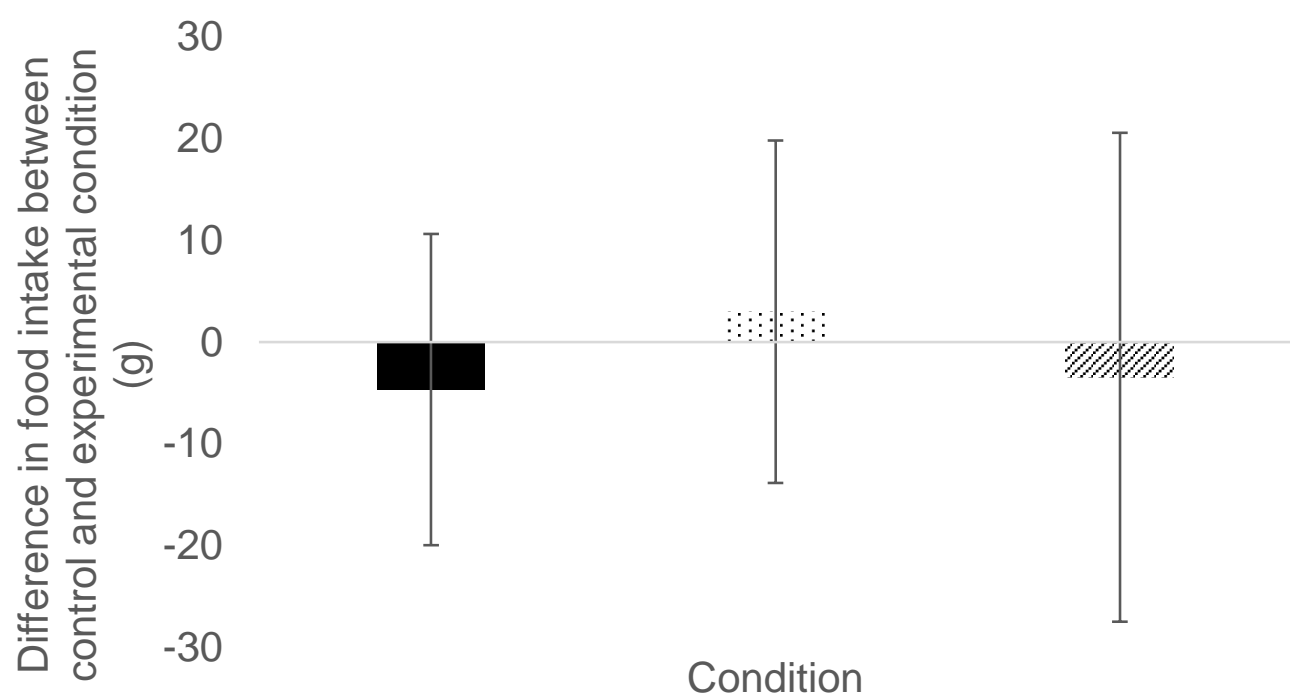

Condition

- Positive :: Negative \# Emotional Confusion

Figure 13. Difference in food intake between the control and experimental condition for the positive, negative and emotional confusing groups controlling for cognitive restraint, hunger and BMI scores. Error bars represent SD.

\subsubsection{Food intake differences across levels of alexithymia}

To explore food intake further, a hierarchical regression was conducted to test the predictive ability of alexithymia on food intake difference scores. Entering the dummy coded group variables in step 1 revealed group allocation explained $3.5 \%$ of the variance in food intake difference scores, but this was not significant, $F(2,118)=$ $2.118, p=.125$. Entering total alexithymia scores did not significantly increase the accuracy of the model (overall model $=F(3,117)=2.449, p=.063)$. Model 2, including group allocation and total alexithymia scores, explained 6\% of the variance in food intake.

\subsubsection{Food intake across high and low emotional eating}

A two-way ANOVA was conducted on the difference scores in food intake across groups and emotional eating scores (two groups: high versus low emotional 
eaters), controlling for hunger, BMI, cognitive restraint and alexithymia. There was no significant main effect of group, $F(2,109)=1.68, p=.191$, or emotional eating, $F(2,109)=.551, p=.46$. There was also no significant group $\mathrm{x}$ emotional eating interaction, $F(2,109)=.86, p=.832$.

\subsubsection{Serial Mediation Model}

\subsubsection{Alexithymia predicting food intake}

The next step in the analysis was to investigate whether alexithymia could predict differences in food intake irrespective of experimental group. Total alexithymia scores were entered as the predictor variable $(X)$ with the difference in food intake as the outcome variable $(Y)$. BDI and NU were entered as mediators and BMI, cognitive restraint, uncontrolled eating, age, and sex were controlled for. The model revealed no significant direct or indirect effects. Similar results were found when BAI scores replaced BDI scores at $M_{l}$.

\subsubsection{Alexithymia predicting emotional eating}

To test the theoretical model developed in Studies One and Two, total alexithymia scores were entered at $X$ and emotional eating (EES and TFEQ separately) at $Y$. Negative affect (BDI and BAI separately) and NU were entered as mediating factors (see Table 13).

When BDI scores were included, total alexithymia scores had a significant direct effect on emotional eating as measured by both the TFEQ, $B=.4518, C I=.1355$ $.7682, p=.006$, and EES, $B=.3191, C I=.0485-.5901, p=.02$. Similar results were found when including BAI scores: TFEQ-EE: $B=3.552, C I=.0369-.6735, p=.029$, and EES: $B=.2791, C I=.0189-.5394, p=.0358$. 
As direct effects were unexpected based on the findings from Study One and Two (Chapter Two), an additional 12 serial mediation models were performed with the subscales of the TAS-20 entered at $X$ (see Table 17).

Table 17. Additional serial multivariate mediation model pathway analysed in Study Four.

\begin{tabular}{ll}
\hline Model & Pathway \\
\hline 1 & DIF $\rightarrow$ BDI $\rightarrow$ NU $\rightarrow$ TFEQ-EE \\
2 & DDF $\rightarrow$ BDI $\rightarrow$ NU $\rightarrow$ TFEQ-EE \\
3 & EOT $\rightarrow$ BDI $\rightarrow$ NU $\rightarrow$ TFEQ-EE \\
4 & DIF $\rightarrow$ BDI $\rightarrow$ NU $\rightarrow$ EES \\
5 & DDF $\rightarrow$ BDI $\rightarrow$ NU $\rightarrow$ EES \\
6 & EOT $\rightarrow$ BDI $\rightarrow$ NU $\rightarrow$ EES \\
7 & DIF $\rightarrow$ BAI $\rightarrow$ NU $\rightarrow$ TFEQ-EE \\
8 & DDF $\rightarrow$ BAI $\rightarrow$ NU $\rightarrow$ TFEQ-EE \\
9 & EOT $\rightarrow$ BDI $\rightarrow$ NU $\rightarrow$ TFEQ-EE \\
10 & DIF $\rightarrow$ BAI $\rightarrow$ NU $\rightarrow$ EES \\
11 & DDF $\rightarrow$ BAI $\rightarrow$ NU $\rightarrow$ EES \\
12 & EOT $\rightarrow$ BAI $\rightarrow$ NU $\rightarrow$ EES \\
\hline BAI Beck
\end{tabular}

$\overline{\mathrm{BAI}}=$ Beck anxiety inventory, BDI = Beck depression inventory, DDF $=$ difficulty describing feelings, DIF = difficulty identifying feelings, EES = emotional eating scale total score, EOT = externally oriented thinking, TFEQ-EE = three factor eating questionnaire emotional eating subscale.

There was no significant direct effect of DIF on emotional eating (Model 1), but a significant indirect effect was found via BDI $\left(a_{1} d_{21} b_{2}\right), B=-.3603, C I=-.8272-$ -.0094. When emotional eating was measured by the EES, DDF had a significant direct 
effect on emotional eating (when controlling for BDI in Model 5: $B=.6814, C I=$ $.0876-1.2752, p=.025$, and when controlling for BAI in the Model 11: $B=.6957, C I$ $=.1234-1.2681, p=.018)$. When emotional eating was measured by the TFEQ, EOT had a significant direct effect on emotional eating (when controlling for BDI in Model 3: $B=.8435, C I=.1873-1.4997, p=.0122$, and when controlling for BAI in Model 9: $B=.7842, C I=.1202-1.4482, p=.021)$. There were no significant direct or indirect effects for Models 2, 4, 6, 7, 8, 10, and 12.

\subsection{Study Four: Discussion}

The overarching aim of Study Four was to explore the effect of mood manipulation (positive, negative, emotional confusion) on food intake. As predicted, overall POMS scores were higher (more positive mood) following positive manipulation of mood compared to the induction of negative and emotionally confusing states. Similar findings were found for the elatedness, composure and clearheadedness subscales of the POMS. In each instance, significantly higher scores (i.e. greater composure) were found following positive manipulation of mood. Taken together, these findings suggest that the novel mood induction procedure was successful in inducing positive mood. However, and in contrast to the pilot data reported in Chapter Three, there were no significant POMS subscale score differences across the emotionally confusing and negative groups. Specifically, the emotionally confusing group did not report significantly lower levels of overall mood (POMS total), composure or clear-headedness. Carry over effects do not seem to offer a viable explanation for these findings as an analysis of order effects did not reveal any significant differences. In addition, average total TAS-20 alexithymia scores were 
higher in the current study (49.99) than in Study Three (44.43). Consequently, DIF (15.8) and DDF (14.72) scores were also higher in the current study (Study Three; DIF: 12.79 , DDF: 12.75). This is important to note as elevated levels of alexithymia may have influenced the manipulation, with participants also experiencing some level of emotional confusion when recalling negative events due to existing difficulties identifying and expressing their emotions. Therefore, this could have reduced differences in mood across the negative and emotionally confusing groups, reducing the likelihood of detecting main effects. However, this seems unlikely as such an effect would also have been observed in the positive condition. It should also be noted that secondary data analysis revealed that all moods returned to, or exceeded, baseline levels following the consumption of food (see Appendix W). This is consistent with previous research reporting that the consumption of food serves as a hedonic reward, with individuals feeling more positive following the consumption of food (e.g. Bongers et al., 2013; Christensen, 2001; Macht \& Mueller, 2007). Overall, the mood manipulation successfully induced a positive emotional state but was not successful in inducing discrete negative and emotional confusing emotional states. These findings contrast with the pilot findings reported in Study Three.

Although Study Three provided support for using autobiographical recall, it is possible that difficulties associated with the task contributed to the lack of significant findings in the current study. Significant changes in the positive group may have been observed as it is easier for a participant to re-experience positive life events in comparison to negative or emotionally confusing events. Support for this is provided in Study Three by the themes generated across the scripts. Participants in the positive group tended to recall achievements and times of celebrations, whereas a broader range of themes were recalled in the negative and emotionally confusing groups. The broader 
range of themes may be an indication that participants found it more difficult to identify an event in which mixed emotions and emotional confusion were experienced because there is a wider range available to them. Additionally, it has been found that over time, the extent to which mixed emotions are experienced decays with delay (Aaker, Drolet, \& Griffin, 2008). For example, Aaker and colleagues (2008) suggested that mixed emotion events are difficult to recall as some individuals may believe a 'decision' should be made concerning the 'overall' emotion experienced. They used the example of a wedding day where the day is positive overall but there may have been additional emotions that have been forgotten (e.g. wedding day nerves, stress of morning preparation, worries about family members meeting). Furthermore, revisiting negative and emotionally confusing events is arguably more difficult and unpleasant than recalling a positive memory. For example, it is more difficult to recall the dates of negative events compared to positive events (Rubin \& Berntsen, 2003), mixed emotion events are more difficult to recall in comparison to single emotions events (e.g. negative or positive; Aaker, Drolet, \& Griffin, 2008), and positive memories are generally rated as being richer in detail (D'Argembeau, Comblain, \& van der Linden, 2003; Ford, Addis, \& Giovanello, 2012). This further illustrates that the negative and emotional confusing groups may have found the task more difficult. In addition, Leist, Ferring and Filipp (2010) found younger adults are less likely to experience negative events. They found that negative events occurred more frequently in middle age and increased in frequency as people age. The current sample were relatively young $(M=$ 24 years) and recalling a negative or emotionally confusing event may have also been difficult, reducing the efficacy of the mood manipulation task.

Overall, Studies Three and Study Four provide mixed results for the use of autobiographical recall to induce feelings of emotional confusion. In Study Three, 
support for the method was found and additional studies have shown that as an internal procedure, it can elicit strong emotional responses (Jallais \& Gilet, 2010). On the other hand, the procedure was not effective in eliciting sufficient changes in negative or emotionally confusing mood states and further exploration into the use of autobiographical recall to induce emotional confusing is therefore necessary. For example, in future studies participants could be encouraged to recall a more recent emotionally confusing event, to explore all aspects of an event thoroughly, or provided with set examples or prompts. In addition, a measure of perceived task difficulty could be included and considered as a potential confounding variable when examining the efficacy of the mood induction paradigm.

The second aim was to test for differences in food intake following the experimental conditions compared to the control condition. As predicted, there were no significant differences in food intake in the control conditions across groups. However, even though the positive and emotionally confusing groups tended to consume less food, and the negative group more, food intake did not statistically differ across groups. Such findings lend support to previous studies that have also found no significant differences in food intake across positive and negative groups in comparison to a control (e.g. Appelhans et al., 2011; Bongers et al., 2013; Haynes et al., 2003). Although, the general trend in food intake observed in the current study is in line with studies that have found an increased intake after a negative mood induction (e.g. Fay \& Finlayson, 2011; Royal \& Kurtz, 2010). Results also revealed no significant differences between high and low emotional eaters and those with or without alexithymia. These findings refute predictions and suggest that higher selfreported eating and greater difficulty understanding emotions are not associated with increased food intake following mood induction tasks. 
One reason for the non-significant results could be due to the range of food items used in the current study. In their meta-analysis, Cardi, Leppanen and Treasure (2015) found that during negative versus control conditions, studies which presented participants with either sweet or savoury foods consumed more than when presented with a choice of both sweet and savoury. In the current study, participants in all conditions were presented with both sweet and savoury foods, and therefore, this may help to explain why observed differences in food intake did not reach statistical significance. (i.e. effects may have been smaller and harder to detect across groups). Cardi, Leppanen and Treasure (2015) also found greater food intake when both sweet and savoury foods were presented during positive versus control mood manipulation. However, this finding was not replicated in the current study as positive mood was associated with a reduction in food intake (although not statistically significant) when using both sweet and savoury foods. Despite potential limitations of using both sweet and savoury snacks, the choice of snacks was based on Price et al. (2016) who found a reduction in consumption following a high construal manipulation. The choice of snacks was also suitable for the use in emotional eating research as they were rated as highly palatable (secondary analysis of the bogus taste test ratings; Baumeister et al., 1994; Oliver et al., 2000). In addition, whole foods were used which may have limited intake for some participants. Higgs and Donohoe (2011) stated that the use of whole foods allows participants to follow pre-existing rules they may have about the consumption of snacks (e.g. only consuming $x$ number of sweets or chocolates). In the current study, whole rather than broken pieces were used due to the type of the snacks and the difficulty that would have been encountered if breaking them up. It is also unlikely this affected the results as studies have shown that when experiencing an emotional state, participants have difficulty balancing their restraint and emotion 
(Greeno \& Wing, 1994). Therefore, it would be expected that the negative and emotionally confusing groups would have consumed more food. Finally, Ganley (1989) advised that depending on the preference of comfort food and the type of food offered, emotional eating may not occur in the laboratory. It may be that the foods participants like to consume following negative affect are not available so an increase in consumption is not found. Possible support for this is Oliver et al. (2000) who used a buffet style presentation of food and found significant results. Therefore, presenting a wider range of snacks could potentially capture the individual and varied preferences of participants more successfully. Future studies may wish to explore the use of different food choices such as all savoury or all sweet highly palatable snacks and choose snacks that can be easily broken into pieces to avoid participants only consuming set amounts. A second consideration is the importance of eating in response to boredom (Koball, Meers, Storfer-Isser, Domoff, \& Musher-Eizenman, 2012). As the control condition was not designed to elicit or cause any emotional arousal, participants may have found the task relatively boring. This may mask the impact of the experimental manipulation or increase food intake during the control condition (Domoff et al., 2014).

The third aim of the current study was to examine the indirect relationship between alexithymia and emotional eating; building on the findings of Study One by expanding existing theoretical models to predict actual food intake. There were no significant direct or indirect effects of alexithymia on actual food intake (as measured by difference in food intake). When exploring the models where self-reported emotional eating was the outcome variable, a number of findings were found. First of all, total alexithymia scores had a significant direct effect on emotional eating as measured by both the TFEQ and EES. Secondly, an indirect effect was found between 
difficulty identifying feelings (DIF) and emotional eating where DIF was associated with increased depression and a reduction in emotional eating. Thirdly, difficulty describing feelings (DDF) had a significant direct effect on emotional eating when measured by the EES, but not TFEQ. Finally, EOT had a significant direct effect on emotional eating as measured by the TFEQ but not the EES. These findings do not provide clarity to the results presented in Chapter Two. Direct effects between the subscales of alexithymia and emotional eating have emerged across the studies presented so far in this thesis, with different effects also observed depending on what measure of emotional eating is used. Differences found in the models when using EES versus TFEQ to measure emotional eating measures may be due to the unique relationship each measure has with the subscales of the TAS-20. Specifically, DDF had a stronger association with EES and the EOT with TFEQ, although this was only small. The EES and TFEQ-EE were only moderately correlated with one another ( $r=$ .51), suggesting that each measure captures distinct features of emotional eating such as: (a) the EES covers a wider range of emotions; (b) the anger/frustration is not examined in the TFEQ-EE, and (c) the TFEQ-EE only one item examines anxiety. This may have resulted in the finding that the anger/frustration and anxiety subscales of the EES had a stronger relationship with DDF than EOT.

The different results across studies may also be due to differing levels of alexithymia and emotional eating across samples. Indeed, higher levels of alexithymia and emotional eating were found in the current study compared to Studies One and Two, which could explain the differences in the observed relationships (e.g. total scores, DDF and EOT showing a significant direct effect, but not DIF). Although, the number of participants at the highest end of the TAS-20 scale (i.e. scoring $>61$ indicating a presence of alexithymia) was similar. The current study also had a 
higher median emotional eating score in comparison to both Study One and Two (secondary analysis). Participants in the current study were classified as high emotional eaters with scores $\geq 52$ whilst high emotional eaters in Study One and Two scored $\geq 46$. This indicates that the models in the previous studies were developed in samples with lower overall levels of emotional eating. It may be that at the more extreme levels of emotional eating, alexithymia plays a more prominent role. Furthermore, the use of a median split for emotional eating should be noted as a potential methodological issue. Studies have found mixed results with the use of a median split to classify participants into high and low emotional eaters (e.g. Evers et al., 2009; Oliver et al., 2000; Wallis \& Hetherington, 2004, 2009). Maxwell and Delaney (1993) state that misclassification using a median split is common and caution should be taken during interpretation. Likewise, van Strien and colleagues (2012) state that emotional eating may be best described as a categorical rather than continuous variable. For example, differences would only be found between extremely low or extremely high emotional eaters. To clarify this, studies would need to be conducted that specifically explore relationships in groups with extreme high and low emotional eating and alexithymia scores.

As with all experimental work within the laboratory setting, caution should be exercised when generalising the results more broadly. Studies conducted in the laboratory lack ecological validity, and therefore, responses in the laboratory may not fully reflect those that would be seen in real life. The experience and response to real negative and stressful events are expected to be more severe than those presented in the laboratory. First, recall of memories in the laboratory may induce emotions of different intensities compared to mood states following recall outside of the laboratory (e.g. real time, diary studies or involuntary recall). The current study supports the idea 
that emotions are experienced at a reduced intensity during recall as participants rated the memories as more intense at the time of the event (through looking at descriptive statistics). Mood induction in the laboratory is also restricted to a short period of time (10 minutes), whereas in real life, emotional experiences last much longer and may even continue for a number of days, or longer. Second, the amount of food consumed inside and outside the laboratory may differ. When individuals are free to consume what they like, it is more likely that individuals may overconsume. Furthermore, within a laboratory setting, social desirability may have influenced the results. It may be that participants ate less overall across all conditions (control versus experimental), which meant any differences in intake were harder to detect. The increase in consumption found in the positive group may also have been comparably reduced.

A promising research design which may overcome some of these problems is ecological momentary assessment (EMA). EMA is a more naturalistic method of capturing emotional experiences and food intake. Through multiple daily assessments, EMA allows the examination of changes to mood and food intake. This enables a more accurate and realistic report of emotions and the response (e.g. food consumed) that follows. Previous research has found that participants are willing and able to engage with the EMA procedure, and EMA allows for additional data to be collected above other methods to allow the exploration of complex theoretical models (Smyth et al., 2001). Smyth and colleagues (2001) also report that EMA is suitable for a wide range of variables (e.g. emotions, eating behaviours, and coping styles) and reduces response bias. Currently, studies have used EMA to explore the associations between negative mood and bulimic symptoms within clinical samples (Crosby et al., 2009; Haedt-Matt \& Keel, 2011; Smyth et al., 2007, 2009; Wegner et al., 2002). Smyth and colleagues (2009) also used EMA to explore specific variations in affect, stress and binge/purge 
behaviours across days and weeks, further highlighting the benefits of EMA across extended periods of time.

Despite mixed findings, the current study was methodologically robust. Indeed, a relative strength of the current study was the use of a within-subjects design where each participant acted as their own control. This ensured food intake following the experimental condition controlled for participant's consumption after a control condition. The conditions were also counterbalanced, and analysis verified that this was successful. Even so, caution still needs to be exercised when generalising the current results beyond individuals with a healthy BMI. Given that previous research has found significant differences in food intake following mood induction in individuals with higher BMI scores (e.g. Jansen et al., 2008; Schneider et al., 2010; Udo et al., 2013), it is important to note that participant's average BMI fell in the normal range for the current study. Therefore, generalising these results to individuals with overweight or obesity may be inappropriate. In addition, the majority of the current sample were female (70\%), and females have been reported to consume less food overall during experimental food intake research (Oliver et al., 2000). Therefore, the high proportion of females in the current study may have resulted in reduced food intake rates overall. In sum, future studies should explore a wide range of BMI scores and ensure there is sufficient representation of male participants.

\subsection{Study Four: Conclusion}

Study Four used the novel mood manipulation paradigm developed in Study Three and assessed food intake following positive, negative and emotionally confusing mood states, with the latter used as an analogue of alexithymia. There were no 
significant differences in food intake across the experimental groups in comparison to a control task. There were also no significant differences when exploring levels of alexithymia and emotional eating. However, alexithymia was significantly and directly related to self-reported levels of emotional eating. Total alexithymia scores and difficulty describing feelings and externally oriented thinking subscale scores had a significant direct effect on emotional eating. This provides further support for the role of alexithymia in emotional eating but given the inconsistency in results across Studies One to Four, the relationship does not appear to be straightforward or easy to interpret. Consequently, it seems likely that additional factors other than negative affect and negative urgency may mediate the relationship between alexithymia and emotional eating.

\subsection{Chapter Four: Key Findings}

- Autobiographical recall is a potentially suitable mood manipulation procedure for the use in eating behaviours.

- Previous literature suggests both positive and negative moods are associated with increased food intake compared to a control task and these differ depending on individual differences such as restraint, obesity and alexithymia.

- No significant differences were found in food intake across the groups (positive, negative and emotionally confusing).

- No significant direct or indirect effects of alexithymia on food intake were observed. 
- Total alexithymia scores and the DDF and EOT subscales had a significant effect on emotional eating, but the relationship differed between the EES and the TFEQ. 


\section{Chapter Five: The relationship between Alexithymia, Emotional Eating, Interoceptive Awareness, and Feeling Fat}

\subsection{Introduction}

So far, this thesis has provided evidence in support for the role of alexithymia in emotional eating. Although, there have been some inconsistencies in the specific pathways of the relationship. To recap, Study One and Two found different indirect effects between alexithymia and emotional eating across the two samples. Specifically, indirect effects via negative urgency alone, negative affect alone and negative affect and negative urgency in serial were found in Study One. Whereas, direct effects between alexithymia and the TAS-20 subscales were found in Study Two. As direct effects were not consistently found, and indirect effects varied across samples, it is possible that additional factors may play a mediating role. To explore this idea further, Chapter Five introduces the concepts of feeling fat and interoceptive awareness. Feeling fat is a common sensation experienced by many individuals and has been found to be associated with a number of different eating behaviours and disorders. Therefore, it was deemed an appropriate concept to explore further due to potential links with emotional eating. In addition, interoceptive awareness, a closely related construct to alexithymia, may also play a role through difficulties attending to and interpreting bodily sensations. Chapter Five will explore feeling fat and interoceptive awareness, incorporating these factors into an inclusive model of emotional eating. It will also present the findings from Study Five which is a two-part study investigating the associations of feeling fat and interoceptive awareness with alexithymia and 
emotional eating. Part one was an online questionnaire and part two was an experimental manipulation of feeling fat.

\subsection{Feeling Fat}

Feeling fat is a common sensation experienced by individuals in the general population, and although present in women who are and are not dieting, it appears to be more frequent and intense in women with eating disorders (Cooper, Deepak, Grocutt, \& Bailey, 2007; Fairburn \& Beglin, 2008). Feeling fat and overweight, as opposed to actually being overweight, has also been associated with poorer psychological wellbeing in adolescents aged 12-13 years of age (Jansen, van de LooijJansen, de Wilde, \& Brug, 2008). Further, feeling fat has been associated with higher levels of affective distress, negative emotions, and negative self-beliefs in women (Cooper et al., 2007)

Feeling fat can be defined as an expression of over-concern for shape and weight, despite no relation to an individual's actual weight or shape, which fluctuates from day to day or even across a day (Fairburn \& Beglin, 2008). Indeed, whilst 'thinking' fat is related to the cognitive component of body image dissatisfaction, 'feeling' fat is thought to relate to the affective component, representing something more than simply being overweight or thinking of oneself as overweight (Tiggemann, 1996). Ben-Tovim and Walker (1991) further define feeling fat as being part of a women's attitude towards her own body, and specifically, how their body is seen as fat by others; a subjective sense of feeling fat. To capture this, Ben-Tovim and Walker (1991) developed the Body Attitudes Questionnaire (BAQ) to explore six aspects of body perceptions, including a subscale targeting the sensation of feeling fat. Within 
this questionnaire, feeling fat is regarded as a multifaceted construct where a number of different factors are thought to act as triggers to such feelings. For example, clothes being tight, food intake, or the intake of certain foods and negative moods.

In addition to such triggers, Fairburn and Beglin (2008) suggest that feeling fat may occur in individuals with eating disorders as the result of mislabelling emotions and certain bodily experiences. Consistent with this, individuals with eating disorders have been reported to experience the sensation of feeling fat within minutes of an upsetting situation (McFarlane, Urbszat, \& Olmsted, 2011). Bruch (1978) also proposed that feeling dissatisfied with one's body and feeling fat may be due to the displacement of negative emotions and feelings. She proposed that negative emotions may be experienced physically as feelings of fat, as individuals may find them easier to deal with as they can have greater control over them (e.g. attempting to lose weight as a way of reducing the feelings of fat; Harper-Giuffre \& MacKenzie, 1992). McFarlane et al. (2011) also explored the theory of body displacement, finding that individuals with eating disorders who were made to feel ineffective by recalling a time where they failed/did something poorly or when someone commented on their ability negatively, reported greater implicit appearance and body concern compared to restrained and unrestrained eaters and those in a control condition.

In a systematic exploration of the features associated with feeling fat, Cooper and colleagues (2007) provide further evidence for a potential contributory role of emotion. Cooper and colleagues (2007) conducted a series of semi-structured interviews with women with $\mathrm{AN}$, women who were dieting and non-dieting women. All participants had felt fat, although individuals with AN had felt fatter and more distressed by feeling fat. Feeling fat was commonly accompanied by negative feelings (e.g. frustration, sadness), although the frequency and intensity of such feelings was 
strongest in the group with AN. All groups reported experiencing both internal (e.g. feeling hot, palpitations, feeling heavy) and external (e.g. clothes feeling tight, waistbands pressing into the stomach) bodily sensations when feeling fat, with internal sensations more prominent in $\mathrm{AN}$ and dieting participants. When participants were asked to discuss their first memories of feeling fat, participants commented on experiencing bodily sensations such as fullness, feeling sick, and butterflies in the stomach. Typical memories associated with first feeling fat also tended to reference negative situations, such as parents trying to control them, being bullied and feeling different. Such findings suggest that following a difficult situation or event, negative emotions and accompanying bodily sensations may be frequently misinterpreted and expressed as feeling fat. Interestingly, no significant correlation was found between BMI and feeling fat, although lower BMI scores were significantly associated with greater distress. In addition, Cooper et al. (2007) speculated that feeling fat is more than simply an expression of the sensations of negative affect, as eating disorderrelated symptoms remained significantly correlated with how fat participants felt even after controlling for levels of depression. Consistent with this, Tiggemann (1996) also pointed to additional contributory factors in the experience of feeling fat in female undergraduate students, including dietary restraint, self-esteem, and depressed affect.

Even so, the above evidence points to an important role of negative situations and resultant emotional distress in the onset and experience of feeling fat. As previously highlighted, this may be due to difficulties understanding one's emotions and regulating negative affect, with feelings of fat expressed as individuals can understand and control these feelings more readily. Therefore, it is possible that any factor limiting a person's ability to identify, describe and regulate emotional distress (i.e. alexithymia) may contribute to feelings of fat. Consistent with this line of 
thinking, Andersen (2000) indirectly highlighted the important role of emotional processing and regulation in feeling fat. He reported that individuals with eating disorders repeatedly voice the complaint, "I feel fat." He stated it was not possible to 'feel fat' and instead the phrase should be, "I think I am fat". He described the word ' $f a t$ ' as a common denominator of many negative emotions, suggesting this ' $f a t$ ' is a ' $g o$ to' feeling when an individual is experiencing negative emotions but is unsure how to approach them. Therefore, during treatment, he recommended that individuals should be asked to replace "I feel fat" with "Ifeel $x$," with the word replacing fat most likely to be the main concern for the individual (e.g. angry, upset or worried). However, this task may prove to be particularly difficult for individuals with alexithymia owing to existing difficulties identifying and describing emotions. Therefore, it is possible that alexithymia may exacerbate one's vulnerability to experiencing sensations of feeling fat as individuals may be more likely to communicate and express emotional distress in a way that is easier for them to understand (i.e. "I feel fat").

Additional evidence for a link between alexithymia and feeling fat comes from the finding that females with alexithymia report greater body checking behaviours and body dissatisfaction (De Berardis et al., 2007). In particular, it was DIF and DDF that played the largest role. In addition, De Berardis and colleagues (2007) also found that individuals with alexithymia experienced greater social anxiety and lower self-esteem. Taken together, this could suggest that individuals with alexithymia may be vulnerable to experiencing greater sensations of feeling fat because they are unable to correctly identify their emotions. They may also be vulnerable to social anxiety and increased body checking behaviours which could result in greater sensations of feeling fat. Therefore, alexithymia may further increase the likelihood of feeling fat as individuals 
with alexithymia may be more likely to misinterpret the perceptual and behavioural aspects of body image. However, a factor that may exacerbate the tendency to report negative emotions as feeling fat may be interoceptive awareness. Interoceptive awareness involves the process of interpreting bodily sensations and is central to how perceptual and behavioural aspects of body image are understood. Consequently, it may be that alexithymia and interoceptive awareness combine to further impair the interpretation of emotions, therefore leading an individual to feel fat.

\subsection{Interoceptive Awareness}

Interoception refers to the accurate perception and interpretation of one's internal bodily sensations (Craig, 2002; Critchley, Wiens, Rotshtein, Öhman, \& Dolan, 2004). Originally the term was used to refer to visceral sensations only (felt in the internal organs, e.g. Cameron, 2002), but its definition has subsequently been expanded to include bodily signals that are not readily considered as internal (e.g. sensual touch and taste which are processed by neural pathways; Craig, 2005). For many years, the perception of bodily signals and sensations have played an important part in theoretical accounts of both emotion (e.g. Damasio, 1994; James, 1884) and emotional eating (e.g. Bruch, 1964; 1973). As discussed in Chapter One (see section 1.4.), individuals who have low interoceptive awareness (i.e. limited awareness of their bodily sensations), may be vulnerable to engaging in emotional eating due to the misinterpretation of bodily sensations of arousal (e.g. butterflies in the stomach associated with anxiety interpreted as pangs of hunger; Young et al., 2017). Studies have also highlighted that interoceptive awareness is related to experiencing a greater 
intensity of emotional experiences (Herbert, Pollatos, \& Schandry, 2007; Pollatos, Herbert, Matthias, \& Schandry, 2007).

Individuals who experience more intense emotions may be unable to effectively regulate them. As previously mentioned throughout Chapters One to Four, emotional dysregulation is thought to play a role in the development and maintenance of emotional eating, and in turn, greater BMI (as seen in the results of Studies One and Two). One potential explanation for this is that poor interoception leads to an atypical perception of bodily signals of one's emotional state, resulting in poor emotional regulation strategies (Murphy, Brewer, Catmur, \& Bird, 2017; Murphy, Catmur, \& Bird, 2018). Interoceptive awareness has been found to be associated with poor mental health and eating behaviours and disorders (Khalsa \& Lapidus, 2016; Klabunde, Acheson, Boutelle, Matthews, \& Kaye, 2013; Murphy et al., 2017; Pollatos et al., 2008; Young et al., 2017).

For example, Young and colleagues (2017) found that emotional eating was significantly and positively related to interoceptive accuracy (the ability to detect interoceptive signals), suggesting that self-reported high emotional eaters are able to accurately detect their bodily signals. However, emotional eating was also negatively associated with interoceptive awareness (defined as their confidence in their ability to perceive bodily sensations), suggesting that even though individuals were able to detect their bodily sensations, they were not confident in their ability to do so. In a second sample, they also found that higher levels of emotional eating were associated with a greater ability to discriminate heartbeat in a detection test, suggesting again that such individuals are able to accurately detect their bodily signals. It could be that high emotional eaters are more attuned to detecting bodily sensations, but as they lack confidence in trusting what they are feeling, they experience difficulties self-regulating 
their sensations. Young and colleagues (2017) also explored the role of interoception in negative affect, finding that interoceptive indices explained $25 \%$ and $36 \%$ of the variance in anxiety and depression ratings, respectively. Interoceptive indices explained $47 \%$ of the variance in emotional eating, and even after controlling for mood, higher interoceptive awareness and lower prediction error (the difference between subjective and objective interoceptive ability) were related to lower levels of emotional eating. Findings also revealed that BMI was significantly and positively associated with interoceptive sensitivity. Such findings are consistent with Herbert, Blechert, Hautzinger, Matthias, and Herbert (2013), who also found that interoceptive sensitivity, the ability to perceive and process bodily sensations, negatively predicted BMI and fully mediated the relationship between BMI and internal hunger cues and eating in response to physical (instead of emotional) cues.

A significant positive relationship between emotional eating (DEBQ) and the EDI-interoceptive awareness subscale (Garner, 1991) has also been reported (van Strien, 2000). Specifically, van Strien (2000) found that interoceptive awareness (EDI) significantly predicted emotional eating; a finding later replicated by Ouwens, van Strien, \& van Leeuwe (2009) when using the DEBQ measure of emotional eating. However, the nature and strength of relationship between emotional eating and interoceptive awareness has differed depending on what measure of emotional eating has been adopted. For example, when measuring emotional eating via the EES, Waller and Osman (1998) found no significant association between interoception and emotional eating in sample of female students.

In addition to emotional eating, interoceptive awareness has also been strongly linked to alexithymia (Gaigg, Cornell, \& Bird, 2018; Garner, 1991; Herbert, Herbert, \& Pollatos, 2011; Longarzo et al., 2015; Murphy et al., 2018; Shah, Hall, Catmur, \& 
Bird, 2016; Taylor, Parker, \& Bagby, 1996). Murphy et al. (2018) conducted a series of experiments exploring the relationship between alexithymia and interoceptive awareness, finding that: (a) individuals with high levels of alexithymia relied on exteroceptive information to judge respiratory output during an interoceptive sensitivity task; (b) higher levels of alexithymia were associated with less accurate perception of interoceptive information, and (c) the relationship between alexithymic traits and interoceptive accuracy was specific to interoception and not due to cooccurring depression and anxiety. Using a heartbeat perception task, a common method to assess interoceptive awareness, Herbert et al. (2011) also found that interoceptive awareness was negatively related to all three subscales of the TAS-20 and levels of depression. Furthermore, interoceptive awareness was an independent and significant predictor of alexithymia total and subscale scores. Herbert et al. (2011) also found that interoceptive awareness was related to the EOT subscale but only in men. It is possible that differences in levels of alexithymia across sexes explain this finding. In addition, the authors suggest that the difference in results may reflect cultural and sex differences in the ideals of coping with emotions and the associated bodily signals.

Most recently, Zamariola, Vlemincx, Luminet, and Corneille (2018) found no significant association between alexithymia and interoceptive accuracy (as measured by a heartbeat perception task) across six studies. Although, they did find significant negative associations between total and subscale scores of the TAS-20 and self-report ratings of interoceptive awareness as measured by the Multidimensional Assessment of Interoceptive Awareness questionnaire (MAIA; Mehling et al., 2012). The strongest associations were found between the TAS-20 and the not-worrying, attention regulation and trusting subscales of the MAIA. The authors suggested that individuals 
with alexithymia may focus less on their bodily sensations but be more sensitive to sensations of pain and discomfort, and experience difficulty trusting their bodily signals. Within an experimental study, van Strien et al. (2014) also found that poor interoceptive awareness was significantly related to self-reported emotional eating. However, they did not find any evidence that poor interoceptive awareness or alexithymia moderated the relationship between hunger stress reactivity (difference in hunger after stress and control condition) and emotional eating and snack intake. In sum, although theoretically linked, the association between alexithymia and interoceptive awareness has received little attention in the research and what literature is available is inconclusive.

Previously, a model has been proposed of overeating which includes emotional, eating, negative affect and interoceptive awareness. This highlights how the factors interact and provides support for exploring them together. Within a dual pathway model of overeating, Stice $(1994 ; 2001)$ proposed that negative affect and restrained eating mediates the link between body dissatisfaction and overeating (see Appendix X). This model was later expanded by van Strien, and colleagues (2005) who suggested that negative affect and overeating were indirectly linked through a lack of interoceptive awareness and emotional eating (see Appendix X). Within a sample of female adolescents, they found that whilst the original dual pathway model fitted the data well, the extended model fit best. In contrast, only the extended model fit the data from their clinical sample (females with AN and/or BN). Overall, they found strong support for the negative affect pathway, but the nature of the indirect pathway differed across samples. In the clinical sample, a positive association between emotional eating and overeating was found, with interoceptive awareness and emotional eating indirectly mediating the relationship between negative affect and 
overeating. Consistent with Bruch (1973), it appears that confusion surrounding bodily sensations related to hunger (e.g. butterflies in the stomach when experiencing nervousness), may result in increased food intake (i.e. emotional eating) in response to negative moods. In contrast, interoceptive awareness did not fully explain the relationship between negative and emotional eating in adolescent females. In a later study, Ouwens, van Strien, van Leeuwe, and van der Staak (2009), the model was further advanced and extended to include food intake. The data from female students fit both models well (Ouwens et al., 2009; Stice, 1994; van Strien et al., 2005), and an indirect relationship between negative affect and consumption via interoceptive awareness and emotional eating was found. Overall, even though these models do not predict emotional eating, they nevertheless provide support for an association between emotional eating, negative affect and interoceptive awareness. As differences were seen in the two female samples (adolescents versus students) further exploration into the relationships is needed. The authors proposed that differences in age may be a contributing factor, but it may also indicate that additional factors, such as feeling fat, may play a role.

In conclusion, in order to successfully regulate one's emotions, it is important to be able to accurately detect and interpret accompanying bodily sensations. Therefore, individuals who are unable to understand their emotions and bodily sensations may be more likely to present with feelings of fat and to engage in emotional eating. Feeling fat is a relatively under researched phenomena but has been found to have an underlying presence across a number of eating behaviours and disorders. It has also been associated with alexithymia, which in turn has also been associated with interoceptive awareness. Consequently, it seems logical to propose 
that both factors may mediate the relationship between alexithymia and emotional eating.

\subsection{Study Five: Aims}

The overarching aim of Study Five was to further explore the mechanisms underpinning emotional eating, and in particular, the role of alexithymia. To explore feeling fat and interoceptive awareness thoroughly, the study was conducted in two parts. Specifically, the first part of Study Five sought to investigate the role of feeling fat and interoceptive awareness as potential mediating factors between alexithymia and emotional eating (see Figure 14). Significant associations between emotional eating, feeling fat and interoceptive awareness were predicted. It was also predicted that difficulty comprehending emotions (alexithymia) and interpreting associated bodily sensations (interoceptive awareness) may lead to sensations of feeling fat, and in turn, emotional eating. This prediction was made as an extension of the previous models of emotional eating presented in this thesis (Chapters Two and Four) and as a further investigation of the role of alexithymia in emotional eating. In line with previous models, alexithymia was entered as the predictor variable where it was proposed that difficulty understanding emotions would lead to increased difficulty processing associated bodily sensations, which in turn, would lead to the sensation of feeling fat (a misinterpretation). 


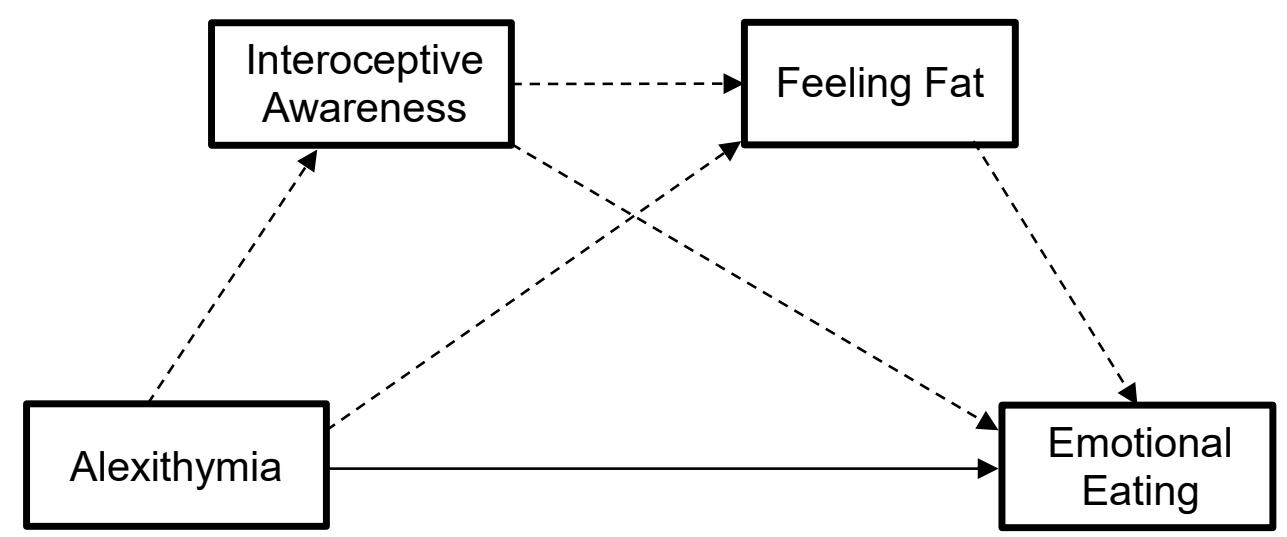

Figure 14. Inclusive model of emotional eating: The proposed direct and indirect relationships between alexithymia and emotional eating including interoceptive awareness and feeling fat.

The second part of the study aimed to examine whether the sensation of feeling fat could be manipulated within a laboratory setting, and in turn, whether it is possible to predict which individuals may be most susceptible to the manipulation of feeling fat. It was predicted that following a negative social comparison manipulation, participants would experience an increase in feeling fat. In contrast, the opposite was expected to occur in response to a positive social comparison. Based on the premise that individuals with alexithymia are vulnerable to social anxiety and increased body checking behaviours, it was also predicted that individuals who experience greater difficulties processing their emotions (evidenced by higher levels of alexithymia), and those who have difficulty perceiving and accurately interpreting their bodily sensations (poor interoceptive awareness) would be more susceptible and vulnerable to feeling fat. 


\subsection{Study Five Methods}

\subsubsection{Participants}

\subsubsection{Part 1 participants}

Participants had to be female and report no history of depression, anxiety or eating disorders to be eligible to take part in the study. The initial sample consisted of 315 participants, with 74 cases subsequently removed (see Table 18).

As being underweight could indicate the presence of an eating disorder, and that eating disorders have been associated with increased sensations of feeling fat (e.g. Fairburn, 2001; Striegel-Moore, McAvay, \& Rodin, 1986), individuals with a BMI below 18.5 were removed. Similarly, participants with a BMI value over 40 (indicating morbid obesity) were also removed because it could be suggestive of disordered eating (Grucza, Przybeck, \& Cloninger, 2007).

Table 18. Reasons for participant exclusions in Study Five.

\begin{tabular}{lc}
\hline Reason & $n$ \\
\hline Did not consent & 7 \\
Duplicate entries & 3 \\
Incomplete questionnaire (accessed but did not complete) & 54 \\
Inaccurate height and weight & 3 \\
BMI scores & 5 \\
a) Below 18.5 & 2 \\
b) Above 40 &
\end{tabular}

The final sample consisted of 241 female participants with a mean age of 24.18 years $(S D=7.58$, range $=18.21-61.10$ years $)$. BMI was available for 164 participants 
$(M=24.04, S D=4.18$, range $=18.51-38.60)$, and if only available via self-report, this was used in the analysis as self-reported BMI was found to be strongly correlated with BMI recorded in the lab $(r=.94, p<.001, N=74)$. As actual weight may play a factor in feeling fat, BMI was explored in greater detail. The percentage of each category as defined by the World Health Organisation (WHO, 2018) was; 'normal weight' $70.1 \%$ $(n=115)$, 'pre-obese' $18.9 \%(n=31)$, 'obesity class I' $8 \%(n=13)$ and 'obesity Class II' $3 \%(n=5)$. An a priori power analysis (G*Power; Faul et al., 2007) indicated that a minimum sample size of 123 was required $($ effect size $=.25-$ medium; power $=.8)$. Therefore, the current study was sufficiently powered $(N=241)$.

\subsubsection{Part 2 participants}

104 participants attended the second session. Mean age was 22.57 years of age $(S D=4.28$, range $=18.46-50.85$ years $)$ and BMI ranged from 18.51 to $38.6(M=24.05$, $S D=4.3)$. Participants were randomly assigned to one of four conditions: negative feeling fat $(n=32)$, positive feeling fat $(n=21)$, negative general $(n=25)$ or neutral $(n=26)$. An a priori power analysis (G*Power; Faul et al., 2007) for a one way ANOVA indicated that a minimum sample size of 25 per condition was required (effect size $=.25$ - medium; power $=.8$ ). Therefore, there was reduced power for some conditions in this part of the study.

\subsubsection{Measures: Part One}

\subsubsection{Demographics}

Individuals were asked to provide their date of birth as part of an anonymised code and age was derived from this information. In the second wave of data collection, 
participants were asked to provide their height and weight to allow BMI to be calculated. Participants who attended part 2 of the study also had their height and weight measured in the laboratory (stadiometer and WW digital scales).

\subsubsection{Toronto Alexithymia Scale}

(TAS-20; Bagby, Parker, et al., 1994; Bagby, Taylor, et al., 1994). As fully described in Chapter Two (section 2.3.2.3.). Cronbach's alphas were as follows for the current study: total alexithymia score $\alpha=.81$, DIF $\alpha=.77$, DDF $\alpha=.74$, and EOT $\alpha=$ .56.

\subsubsection{Body Attitudes Questionnaire}

(BAQ; Ben-Tovim \& Walker, 1991). The BAQ is a 44-item measure assessing the broad range of attitudes individuals, particularly women, hold about their bodies. The BAQ consists of six subscales (self-disparagement, strength, salience of weight, feelings of overall fatness, feelings of attractiveness and consciousness of lower body fat), although only the feeling fat (FF) subscale was used in the current study. The feeling fat subscale consists of 14 items that measure the extent to which an individual experiences the sensations of feeling fat. For example, "Eating sweets, cakes or other high calorie food, makes me feel fat". Participants respond by selecting the answer that best describes them on a 5-point scale from 1 ("strongly disagree") to 5 ("strongly agree"), with higher scores indicative of greater sensations of feeling fat. In line with previous studies (Paxton \& Phythian, 1999; Schutz \& Paxton, 2007), internal consistency of the FF subscale was good in the current study $(\alpha=.88)$.

\subsubsection{Multidimensional Assessment of Interoceptive Awareness}

(MAIA; Mehling et al., 2012). The MAIA is a 32-item measure assessing interoceptive body awareness across eight subscales: noticing, defined as awareness of uncomfortable, comfortable and neutral body sensations $(\mathrm{N} ; 4$ items, e.g. "When I 
am tense I notice where the tension is located in my body," $\alpha=.54)$; not-distracting which is the tendency to not ignore or distract oneself from sensations of pain or discomfort (ND; 3 items, e.g. "When I feel pain or discomfort, I try to power through $i t^{\prime \prime}, \alpha=.39$ ); not-worrying, the tendency to not worry or experience emotional distress with sensations of pain or discomfort (NW; 3 items, "When I feel physical pain, I become upset", $\alpha=.50)$; attention regulation which is the ability to sustain and control attention to body sensations (AR; 7 items, e.g. "I can return awareness to my body if I am distracted", $\alpha=.79$ ); emotional awareness, defined as the awareness of the connection between bodily sensations and emotional states (EA; 5 items, e.g. "I notice how my body changes when I am angry", $\alpha=.81$ ); self-regulation which is the ability to regulate distress by attention to body sensations (SR; 4 items, e.g. "When I feel overwhelmed I can find a place inside", $\alpha=.78$ ); body listening which refers to active listening of the body for insight (BL; 3 items, e.g. "When I am upset, I take time to explore how my body feels", $\alpha=.82$ ), and finally, trusting, defined as experiencing one's body as safe and trustworthy (T; 3 items, e.g. "I am at home in my body", $\alpha=$ .80). Participants respond by selecting the answer that best describes them on a 5-point scale from 1 ("never") to 5 ("always") and subscales are calculated by taking the average of all items within the subscale. Higher scores are indicative of greater interoceptive awareness. The original MAIA (Mehling et al., 2012) was scored from 0 to 5 , but due to an error when inputting the questionnaire into the online survey, the current study adopted a 1 to 5 scale. Reassuringly, the majority of Cronbach's alphas were good and consistent with levels reported in previous studies and even the subscales with lower alphas (noticing, not-distracting and not-worrying subscales) were comparable to those reported in other samples (Young et al., 2017; Zamariola et al., 2018). 


\subsubsection{Dutch Eating Behaviour Questionnaire}

(DEBQ; van Strien, Frijters, Bergers, \& Defares, 1986). Formulated from the psychosomatic, externality and restraint eating theory, the DEBQ is a 33-item measure assessing three aspects of eating behaviour: emotional eating, external eating and cognitive restraint. The emotional eating subscale (DEBQE; 13 items, e.g. "Do you have a desire to eat when you are feeling lonely?" $\alpha=.92$ ) relates to eating in response to specific and diffuse emotions (a term used to encompass broad emotional states; e.g. nothing to do, bored or restless). The external eating subscale (DEBQX, 10 items, e.g. "If food smells or looks good, do you eat more than usual?" $\alpha=.82$ ) refers to eating in response to food related stimuli regardless of internal state or hunger. Participants respond by selecting the answer that best describes them on a 5-point Likert type scale from 1 ("never") to 5 ("very often"). The DEBQ was used to further explore emotional eating and to provide validity to the original model (Study One and Two). In addition, as the current study explored the ability to accurately perceive and interpret bodily sensations, the DEBQ was favoured over the EES and TFEQ as it incorporates both internal (emotional) and external eating behaviours (the latter being used as part of an additional study outside of this thesis). The psychosomatic and externality theories both involve an individual's misperception of one's internal state prior to eating (Robbins \& Fray, 1980). The restrained eating subscale (DEBQR; 10 items, e.g. "Do you take into account your weight with what you eat?" $\alpha=.92$ ) refers to measures taken to control intake to maintain or reduce body shape and weight.

\subsubsection{UPPS-P}

(Cyders et al., 2007; Whiteside \& Lynam, 2001). As fully described in Chapter Two (section 2.3.2.4.). Internal consistency was found to be good in the current 
sample: NU $\alpha=.76$, PREM $\alpha=.85$, SS $\alpha=.80$, PERS $\alpha=.77$, PU $\alpha=.87$ and total impulsivity scores $\alpha=.89$.

\subsubsection{Other Measures}

In addition to the above, participants also completed the Multidimensional Perfectionism Scale (MPS; Hewitt, Flett, Turnbull-Donovan, \& Mikail, 1991) and the Physical Appearance Comparison Scale - revised (PACS-R; Schaefer \& Thompson, 2014) in part one. However, a full description of these measures is not provided here as they were not used as part of the analysis in this thesis.

\subsubsection{Measures: Part Two}

\subsubsection{Positive Affect and Negative Affect Scale}

(PANAS; Watson, Clark, \& Tellegen, 1988). As fully described in Chapter Two (section 2.3.2.2). Internal consistency for the current study was PA $\alpha=.85 ; .88$ and NA $\alpha=.84 ; .88$ for T1 and T2 respectively. The PANAS was used as a pre- and post-manipulation measure of mood. Due to the total number of measures included in the two parts of the study, anxiety and depression were not measured separately to prevent the study from becoming too onerous for participants.

\subsubsection{Visual Analogues scales}

Visual analogue scales (VAS) were used in part two to check the manipulation was successful. Before and after the feeling fat manipulation, participants were asked to indicate how comfortable, aware of bodily sensations, fat, confident, tired and attractive they felt right now on a scale of 0 to 100 ("not at all" to "extremely"). The main dependent variables were how fat, aware, and attractive they felt. The remaining 
three items (comfortable, tired and confident) were included as part of the cover story and to distract participants from the main aims of the study.

\subsubsection{Heartbeat Detection Task}

Participants completed a heartbeat perception task according to the Mental Tracking Method (Schandry, 1981). For full information see Young et al. (2017). Participants completed this once before and after the manipulation. Analysis of this data is not included in this thesis.

\subsubsection{Design}

Part one of the study was an online survey with part two requiring participants to attend a session in the laboratory. Part two adopted an independent measures design with four groups; positive food situation (Pos-FF), negative food situation (Neg-FF), neutral food situation (Neut.) and a negative social situation (Neg-SS; see Appendix $\mathrm{AB}$, for details). The negative social situation was included to explore whether feeling fat was related to a food/body awareness situation specifically, or whether experiencing negative emotions more generally could also have an effect on sensations of feeling fat.

\subsubsection{Procedure}

Ethical approval was obtained from the Department of Psychology Ethics Committee, College of Human and Health Sciences, Swansea University.

Participants were recruited through the Department of Psychology's participant pool in exchange for course credit, or through adverts/flyers in which 
participants received payment. Psychology students received two course credits for the completion of part one and an additional two credits for attending the laboratory session. Non-psychology students and members of the general public received $£ 5$ in exchange for completing both parts of the study. The study was advertised as "An investigation into the relationship between personality traits and body image in females", with the laboratory session focusing on physiological awareness in social situations. Participants were presented with an electronic information page and consent form at the start of part one via the online survey software Qualtrics (Qualtrics, Provo, UT). Participants who agreed to take part were asked to generate a unique identifier (initials and date of birth; e.g. AP18081992), so their data was anonymous but could be matched across both parts of the study. In part one, participants completed the TAS20, BAQ-FF subscale, MAIA, DEBQ, UPPS-P, MPS, PACS-R and provided their height and weight. Part one took up to 30 minutes to complete. At the end, participants were thanked for their time, debriefed and asked if they would like to take part in the second part of the study.

Participants that agreed to participate in part two were asked to attend a session in the laboratory, scheduled to take place at least one week following part one. Participants were asked to read a second information sheet and complete a consent form. Participants were informed that the study was exploring individual's physiological awareness of social situations and mood. Participants completed the PANAS and VAS before the first heartbeat perception task. Following this, participants were randomly selected to be in one of four groups. The instructions provided were as follows, "Take a few minutes to read the scenario and try to imagine yourself as experiencing the scenario. Write in the text box a few sentences on how you might feel or what you might think in this situation". Participants were then 
presented with a vignette and given time (undefined) to complete the task before completing the PANAS, VAS and the heartbeat perception task for a second time. Before leaving, participants had their height and weight measured. Participants were then thanked for their time and presented with a debrief sheet. The second part of the study took up to 30 minutes to complete.

\subsubsection{Statistical Analysis}

\subsubsection{Part one}

All analysis was conducted using SPSS 22.0. Initial Pearson correlation analysis was conducted on all variables: TAS-20, MAIA, DEBQ, BAQ-FF, NU and BMI. Bonferroni adjusted alpha levels were used where appropriate to control for the possibility of inflated type 1 owing to multiple comparisons.

Serial mediation models (PROCESS 2.16.3; Model 6) were conducted to explore the role of feeling fat and interoceptive awareness as potential mediators in the relationship between alexithymia and emotional eating. As before, TAS-20 total and subscale scores were entered as predictor variables at $X$, with emotional eating as measured by the DEBQ as an outcome variable at $Y$ (see Table 19).

Based on significant associations with feeling fat in the correlational analysis (see section 5.6.2.2.), the trusting, noticing and self-regulation MAIA subscale were entered at $M_{1}$. Feeling fat was entered at $M_{2}$. Analysis was conducted with bootstrapping 10,000 resamples and generated accelerated $95 \%$ confidence intervals. Contrast analysis between multiple significant specific indirect pathways was also conducted where appropriate. 
Table 19. Serial multivariate mediation pathways analysed in Study Five.

\begin{tabular}{|c|c|}
\hline Model & Pathway \\
\hline 1 & TAS-20 $\rightarrow$ MAIA-T $\rightarrow$ FF $\rightarrow$ DEBQ-E \\
\hline 2 & TAS-20 $\rightarrow$ MAIA-N $\rightarrow$ FF $\rightarrow$ DEBQE \\
\hline 3 & TAS-20 $\rightarrow$ MAIA-SR $\rightarrow$ FF $\rightarrow$ DEBQE \\
\hline 4 & $\mathrm{DIF} \rightarrow \mathrm{MAIA}-\mathrm{T} \rightarrow \mathrm{FF} \rightarrow \mathrm{DEBQE}$ \\
\hline 5 & $\mathrm{DDF} \rightarrow \mathrm{MAIA}-\mathrm{T} \rightarrow \mathrm{FF} \rightarrow \mathrm{DEBQE}$ \\
\hline 6 & $\mathrm{EOT} \rightarrow \mathrm{MAIA}-\mathrm{T} \rightarrow \mathrm{FF} \rightarrow \mathrm{DEBQE}$ \\
\hline \multicolumn{2}{|c|}{$\begin{array}{l}\text { DDF = difficulty describing feelings, DEBQE = emotional eating subscale of the } \\
\text { DEBQ, DIF = difficulty identifying feelings, EOT = externally oriented thinking, FF } \\
\text { = feeling fat, MAIA-N = noticing subscale of the MAIA, MAIA-SR = self-regulation } \\
\text { subscale of the MAIA, MAIA-T = trusting subscale of the MAIA, TAS-20 = total } \\
\text { alexithymia scores. }\end{array}$} \\
\hline
\end{tabular}

\subsubsection{Part two}

As a manipulation check, difference scores were calculated by subtracting time $1\left(T_{2}\right.$; pre-manipulation) scores from time $2\left(T_{1}\right.$ : post-manipulation) scores where a higher value indicated an increase. The variables of interest were ratings of how fat participants felt, how aware of their body they were and how attractive they felt. Difference scores were then entered into a one-way ANOVA to check for differences across groups. Difference scores were also calculated for PA and NA and a one-way ANOVA was conducted to explore changes in mood.

A moderation analysis (PROCESS 2.16.3, Model 1) was conducted to test whether personality traits (interoceptive awareness, feeling fat and negative urgency) in part one moderated the relationship between alexithymia and differences in feeling fat scores irrespective of group. Difference scores in feeling fat were collapsed across all groups. Total alexithymia scores were entered as the predictor variable $(X)$ and difference score in feeling fat was entered as the outcome variable $(Y)$. The subscales of the MAIA, FF and NU were used as moderating factors at $M$. Analysis used 
bootstrapping with 10,000 resamples (seed $=9456$ ). In addition, variables were mean centred and Johnson-Neyman technique was used to identify significant regions. PROCESS was also used to generate data in order to plot the moderation figures (see Figures 16 and 17).

\subsection{Study Five: Part One Results}

\subsubsection{Descriptive Statistics}

Descriptive statistics for Study Five Part One can be seen in Table 20.

\subsubsection{Correlational Analysis}

\subsubsection{Correlations between emotional eating, feeling fat, alexithymia and} BMI

Emotional eating and FF scores were significantly and positively correlated with DIF, DDF and total alexithymia scores (see Table 21). FF was also significantly positively correlated with BMI $(r=.296, p<.001)$ and emotional eating $(r=.361, p<.001)$. With the exception of the correlation between DDF and emotional eating scores $(r=.178, p=.006)$, all correlations remained significant after applying a Bonferroni adjusted alpha level of $\mathrm{p}<.005)$. No significant correlations were found between alexithymia and BMI. 
Table 20. Mean, standard deviation and range for each study variable in Study Five Part One.

\begin{tabular}{lccc}
\hline & Mean & SD & Range \\
\hline DIF & 16.58 & 5.32 & $7.00-32.00$ \\
DDF & 12.79 & 4.26 & $5.00-25.00$ \\
EOT & 19.24 & 4.47 & $9.00-33.00$ \\
TAS-20 & 48.62 & 10.77 & $25.00-78.00$ \\
FF & 43.65 & 10.46 & $13.00-65.00$ \\
MAIA-N & 3.51 & .70 & $1.50-5.00$ \\
MAIA-ND & 2.76 & .70 & $1.00-500$ \\
MAIA-NW & 3.04 & .76 & $1.00-5.00$ \\
MAIA-AR & 3.16 & .67 & $1.43-4.86$ \\
MAIA-EA & 3.46 & .85 & $1.00-5.00$ \\
MAIA-SR & 3.08 & .87 & $1.00-5.00$ \\
MAIA-BL & 2.70 & .97 & $1.00-5.00$ \\
MAIA-T & 3.19 & .91 & $1.00-5.00$ \\
DEBQ-EE & 2.89 & .89 & $1.00-5.00$ \\
DEBQ-XE & 3.29 & .64 & $1.40-5.00$ \\
DEBQ-R & 2.86 & .89 & $1.00-5.00$ \\
NU & 29.19 & 5.76 & $16.00-44.00$ \\
\hline DDF & & .89 & 000 \\
\hline
\end{tabular}

DDF = difficulty describing feeling, DEBQ-E = emotional eating DEBQ subscale, DEBQ-R = restraint DEBQ subscale, DEBQ-XE = external eating DEBQ subscale, $\mathrm{DDF}=$ difficulty describing feelings, EOT $=$ externally oriented thinking, $\mathrm{FF}=$ feeling fat, MAIA = Multidimensional Assessment of Interoceptive Awareness, $\mathrm{N}=$ noticing, $\mathrm{ND}=$ non-distracting, $\mathrm{NW}=$ not-worrying, $\mathrm{AR}=$ attention regulation, $\mathrm{EA}=$ emotional awareness, $\mathrm{SR}=$ self-regulation, $\mathrm{BL}=$ body listening, $\mathrm{T}=$ Trusting, $\mathrm{NU}=$ negative urgency, TAS-20 $=$ total Toronto Alexithymia Scale scores. 
Table 21. Pearson correlations between emotional eating, feeling fat, alexithymia and BMI.

\begin{tabular}{lccccc}
\hline & DIF & DDF & EOT & TAS-20 & BMI \\
\hline DEBQ-EE & $.271 * * *$ & $.178^{* * *}$ & -.032 & $.191 * * *$ & -.035 \\
FF & $.235^{* * *}$ & $.265^{* * *}$ & .013 & $.226 * * *$ & $.296 * * *$ \\
\hline
\end{tabular}

$\mathrm{BMI}=$ body mass index, DDF = difficulty describing feelings, DEBQ-EE = emotional eating DEBQ subscale, DIF = difficulty identifying feelings, EOT = externally oriented thinking, FF $=$ feeling fat, TAS $-20=$ total Toronto Alexithymia Scale scores. $* * *=p<.001$.

\subsubsection{Correlations between emotional eating, feeling fat, interoceptive}

\section{awareness and BMI}

Emotional eating was significantly correlated to the not worrying (NW: $r=-$ $.164, p=.010)$, emotional awareness (EA; $r=.142, p=.027)$, and trusting (T: $r=-$ $.138, p=.032$ ) subscales of the MAIA. Feeling fat was significantly correlated with three of the MAIA subscales: $\mathrm{N}: r=.213, p=.001$; SR: $r=-.152, p=.018$, and T: $r=$ $-.406, p<.001$. In addition, BMI was significantly and negatively correlated with body listening $(r=-161, p=.04)$. However, the correlations between feeling fat, noticing and trusting were the only two to remain significant following adoption of a Bonferroni adjusted alpha level $(p<.002$; see Table 22).

\subsubsection{Correlations between alexithymia and interoceptive awareness}

Several significant correlations were found between alexithymia scores and the subscales of the MAIA (see Table 23). The strongest correlations were found between the trusting subscale of the MAIA and total $(r=-.337, p<.001)$ and DIF $(r=-.313, p$ $<.001)$ alexithymia scores. All correlations marked with *** in Table 23 remained significant after adopting a Bonferroni adjusted alpha level $(p<.002)$ 
Table 22. Pearson correlations between emotional eating, feeling fat, BMI and interoceptive awareness (MAIA).

\begin{tabular}{lcccccccc}
\hline & $\mathrm{N}$ & $\mathrm{ND}$ & $\mathrm{NW}$ & $\mathrm{AR}$ & $\mathrm{EA}$ & $\mathrm{SR}$ & $\mathrm{BL}$ & $\mathrm{T}$ \\
\hline DEBQE & .109 & -.077 & $-.161^{*}$ & .011 & $.138^{*}$ & -.009 & .047 & $-.138^{*}$ \\
$\mathrm{FF}$ & $.213^{* *}$ & -.078 & -.123 & -.014 & .001 & $-.152^{*}$ & -.081 & $-.406^{* *}$ \\
$\mathrm{BMI}$ & .115 & .124 & .079 & .132 & -.123 & -.087 & $-.161^{*}$ & -.061 \\
\hline
\end{tabular}

$\overline{\mathrm{AR}}=$ attention regulation, $\mathrm{BMI}=$ body mass index, $\mathrm{BL}=$ body listening, $\mathrm{DEBQ}-\mathrm{EE}$ $=$ emotional eating DEBQ subscale, $\mathrm{EA}=$ emotional awareness, $\mathrm{FF}=$ feeling fat, $\mathrm{N}=$ noticing, $\mathrm{ND}=$ non-distracting, $\mathrm{NW}=$ not-worrying, $\mathrm{SR}=$ self-regulation, $\mathrm{T}=$ trusting. $*=\mathrm{p}<.05, * *=\mathrm{p}<.01$.

Table 23. Pearson correlations between alexithymia and interoceptive awareness (MAIA).

\begin{tabular}{lllll}
\hline & DIF & DDF & EOT & TAS-20 \\
\hline $\mathrm{N}$ & -.012 & -.113 & $-.211^{* * *}$ & $-.138^{*}$ \\
$\mathrm{ND}$ & -.068 & -.047 & -.019 & -.06 \\
$\mathrm{NW}$ & $-.189 * *$ & -.021 & -.011 & -.106 \\
$\mathrm{AR}$ & $-.155^{*}$ & $-.189 * *$ & $-.170^{* *}$ & $-.222^{* *}$ \\
$\mathrm{EA}$ & .019 & $-.182^{* *}$ & $-.272^{* * *}$ & $-.175^{* *}$ \\
$\mathrm{SR}$ & -.089 & $-.127^{*}$ & $-.205^{* * *}$ & $-.179 * * *$ \\
$\mathrm{BL}$ & .028 & -.084 & $-.216^{* * *}$ & -.109 \\
$\mathrm{~T}$ & $-.313^{* * *}$ & $-.281^{* * *}$ & $-.172^{* *}$ & $-.337 * * *$ \\
\hline $\mathrm{AR}$ & &
\end{tabular}

$\mathrm{AR}=$ attention regulation, $\mathrm{BL}=$ body listening, $\mathrm{DIF}=$ difficulty identifying feelings, DDF $=$ difficulty describing feelings, EA $=$ emotional awareness, EOT $=$ externally oriented thinking, $\mathrm{N}=$ noticing, $\mathrm{ND}=$ non-distracting, $\mathrm{NW}=$ not-worrying, $\mathrm{SR}=$ selfregulation, TAS-20 $=$ total Toronto Alexithymia Scale scores, $\mathrm{T}=$ trusting. $*=p<$ $.05, * *=p<.01, * * *=p \leq .001$.

\subsubsection{Correlations between interoceptive awareness, feeling fat and negative urgency}

Negative urgency was significantly positively correlated with FF, $r=.333, p<$ .001 , and negatively correlated with the majority of subscales from the MAIA (NW: $r$ $=-.249, p<.001 ; \mathrm{AR}: r=-.150, p=.02 ; \mathrm{T}: r=-.265, p<.001 ; \mathrm{N}: r=.129, p=.046$. 
However, the latter did not remain significant after Bonferroni correction, $p<.005$. A significant positive correlation was also found between negative urgency and DEBQ scores, $r=.366, p<.001$, but there was no significant association with BMI $(r=-.116$, $p=.140)$.

\subsubsection{Serial Mediation Analysis}

Serial mediation analysis of Model 1 (see Table 19) found a significant direct effect of total alexithymia scores on emotional eating ( $\left.c^{\prime}\right), B=.0105, C I=.0001-.0209$, $p=.048$ as well a significant specific indirect effect via MAIA trusting and feeling fat $\left(a_{1} d_{21} b_{2}\right), B=.0036, C I=.0017-.0068$. Entering MAIA noticing at $M_{l}$ (Model 2) also revealed a significant direct effect of total alexithymia scores on emotional eating $\left(c^{\prime}\right)$, $B=.0104, C I=.0002-.0206, p=.045$ and there was a significant specific indirect effect via feeling fat $\left(a_{2} b_{2}\right), B=.0068, C I=.0034-.0116$. Model 3 (MAIA selfregulation at $M_{3}$ ) found a significant direct effect of total alexithymia scores on emotional eating ( $\left.c^{\prime}\right), B=.0103, C I=.0002-.0204, p=.045$, and a significant specific indirect effect via feeling fat $\left(a_{2} b_{2}\right), B=.0058, C I=.0022-.0105$ and a second indirect effect approached significance via MAIA self-regulation and feeling fat $\left(a_{1} d_{21} b_{2}\right), B=$ $.0006, C I=.0000-.0020$.

To further explore the significant effects found via MAIA trusting, the models were analysed with the TAS-20 subscales entered as $X$ for Models 4-6. Model 4 found a significant direct effect of DIF on emotional eating $\left(c^{\prime}\right), B=.0354, C I=.0148-.0559$, $p<.001$ and a significant specific indirect effect via MAIA trusting and feeling fat $\left(a_{1} d_{21} b_{2}\right), B=.0065, C I=.0030-.0125$. When DDF and EOT were entered as $X$, no significant direct effect was found (Models 5-6). Model 5 (DDF as $X$ ) had two 
significant specific indirect effects: via MAIA trusting and feeling fat $\left(a_{1} d_{21} b_{2}\right), B=$ $.0073, C I=.0032-.0142$, and via the second via feeling fat $\left(a_{2} b_{2}\right), B=.0118, C I=$ .0037-.0223. Model 6 (EOT as $X$ ) revealed a significant specific indirect effect via MAIA trusting and feeling fat $\left(a_{1} d_{21} b_{2}\right), B=.0052, C I=.0014-.0112$.

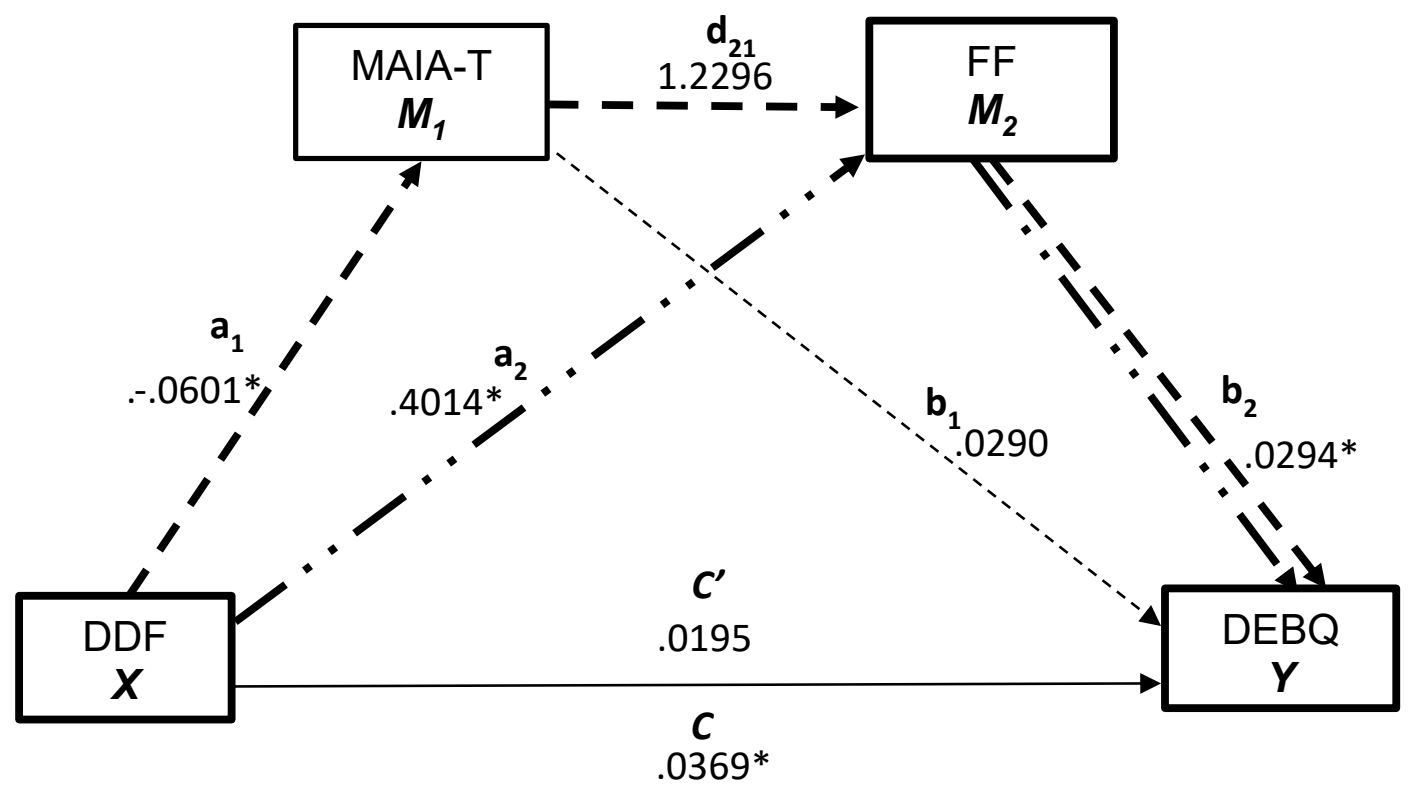

Figure 15. Example PROCESS serial multiple mediation output to identify the direct and indirect effects between difficulty describing feelings (DDF) and emotional eating (DBEQ) including MAIA trusting (MAIA-T) and feeling fat (FF) as mediators.

\subsection{Study Five: Part Two Results}

\subsubsection{Manipulation check}

To test whether the feeling fat manipulation was successful, a series of oneway ANOVA's examining the difference scores between $T_{1}$ and $T_{2}$ across groups for feeling fat, feelings of attractiveness and awareness of bodily sensations were conducted. No significant between groups differences were found (feeling fat: $F(3,98)$ 
$=.566, p=.639$; feelings of attractiveness: $F(3,97)=.703, p=.552$, and awareness: $F(3,99)=0.49, p=.986)$. As the manipulation appeared to have been unsuccessful, additional analysis was performed, revealing no significant between groups difference for confidence, $F(3,99)=1.85, p=.144$ or tiredness scores, $F(3,96)=.482, p=.695$. However, a significant between groups difference was found on the difference scores for the comfortable VAS, $F(3,98)=2.837, p=.042, \eta^{2}=.080$. Pairwise comparisons revealed that difference scores in the Neg-FF group $(M=-6.39, S D=19.28)$ were significantly higher than the Neut. group $(M=5.65, S D=15.45, p=.040)$. That is, participants in the Neg-FF group reported feeling more uncomfortable following the manipulation than those in the Neut. group. No other pairwise comparisons were significant, and therefore, the results suggest that the manipulation was unsuccessful overall.

\subsubsection{Moderation Analysis}

Moderation analysis was conducted to test whether the subscales of the MAIA, FF and NU, moderated the relationship between alexithymia and differences in feeling fat scores irrespective of group. MAIA trusting $(\mathrm{T})$, noticing $(\mathrm{N})$, not worrying $(\mathrm{NW})$ FF and NU did not result in significant predictor models (MAIA-T: $F(3,98)=1.47, p$ $=.23$, MAIA-N: $F(3,98)=.359, p=.783$, MAIA-NW: $F(3,98)=.501, p=.683$, FF: $F(3,98)=.49, p=.69, \mathrm{NU}: F(3,98)=1.03, p=.38)$.

For MAIA attention regulation (AR), the overall model was significant, $F$ $(3,98)=3.32, p=.023$. MAIA-AR was not a unique significant predictor of differences in feeling fat scores, $b=2.181, t(98)=1.611, p=.111$, neither was total alexithymia scores (TAS-20), $b=.13, t(98)=1.609, p=.111$. The MAIA-AR x TAS-20 interaction 
was significant, $b=.29, t(98)=2.576, p=.012$. When the value of MAIA-AR was 3.28 or above, TAS-20 and differences in feeling fat scores were significantly related, $b=.165, t(98)=1.984, p=.05$. As the value of MAIA-AR increases, the relationship between TAS-20 and differences in feeling fat becomes more positive, with the highest attention regulation value (4.64) having the strongest relationship, $b=.559, t(98)=$ $2.909, p=.005$. At low levels of alexithymia, similar changes in feeling fat occur. In contrast, the greatest increase in feeling fat occurs when there are high levels of both alexithymia and attention regulation. Here, individuals may have greater difficulty identifying their bodily sensations but may be more able to attend to them. Therefore, individuals report higher levels of feeling fat because although they are aware they are experiencing bodily sensations; they are unsure what they are experiencing and interpret them as feeling fat. At low levels of attention regulation and high levels of alexithymia, difficulty processing emotions appears to have less impact as they are less likely to attend to the sensations.

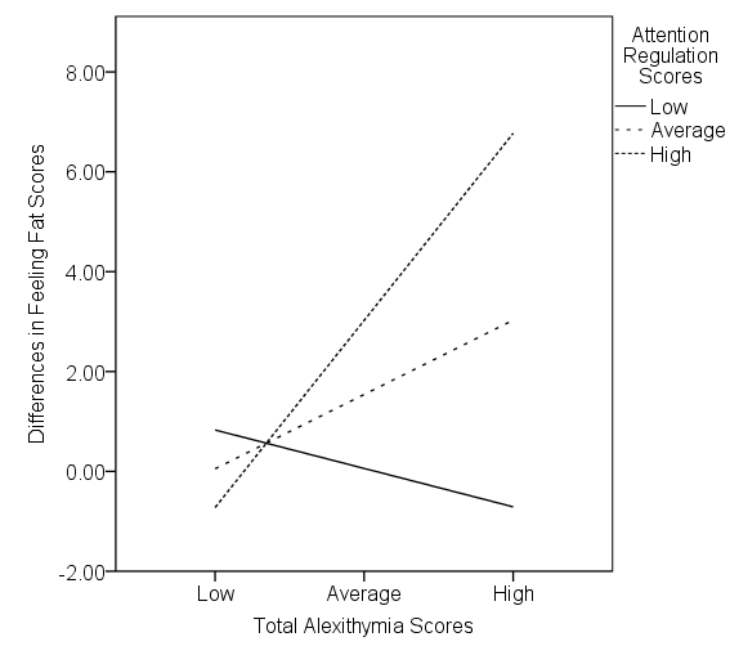

Figure 16. The interactions between total alexithymia and attention regulation scores for differences in feeling fat scores. 
The overall model for MAIA emotional awareness (EA) was also significant, $F(3,98)=3.286, p=.024$. Neither MAIA-EA, $b=-1.034, t(98)=-.9979, p=.321$, or TAS-20, $b=.034, t(98)=.441, p=.661$ scores were a unique significant predictor of differences in feeling fat scores. The MAIA-EA x TAS-20 interaction was significant, $b=.249, t(98)=2.858, p=.005$. For MAIA-EA values of 2.23 and below, the TAS20 and differences in feeling fat scores were significantly related, $b=-.264, t(98)=-$ 1.985, $p=.05$. As the value of emotional awareness decreases, the relationship between TAS-20 and differences in feeling fat becomes more negative, with the lowest emotional awareness value (.862) having the strongest relationship, $b=-.613, t(98)=$ $-2.503, p=.014$. For MAIA-EA values of 4.03 and above, the TAS-20 and differences in feeling fat scores were significantly related, $b=.177, t(98)=1.985, p=.05$. As the value of emotional awareness increases, the relationship between TAS-20 and differences in feeling fat becomes more positive, with the highest emotional awareness value (4.86) having the strongest relationship, $b=.385, t(98)=2.742, p=.007$. The largest increase in feeling fat scores are found when there are low levels of both alexithymia and emotional awareness. Here, individuals have a good ability to understand their emotions but a poor ability to detect the connection between bodily sensations and emotional states. Therefore, as individuals experience emotions, they are unable to associate them with their bodily sensations, and in turn, experience feeling fat. In contrast, the biggest decrease in feeling fat occurs when alexithymia scores are low and emotional scores are high. This suggests that feeling fat is less likely to occur when individuals are able to successfully connect their bodily sensations with their emotional states and possess good ability to understand their emotions. At high levels of alexithymia and high levels of emotional awareness, individuals have difficulty understanding their emotions but are able to appropriately associate their 
bodily sensations with their emotions. It could be that such individuals believe they are good at connecting their bodily sensations with emotional states, but owing to difficulties identifying emotions, they associate feeling fat with their bodily sensations incorrectly.

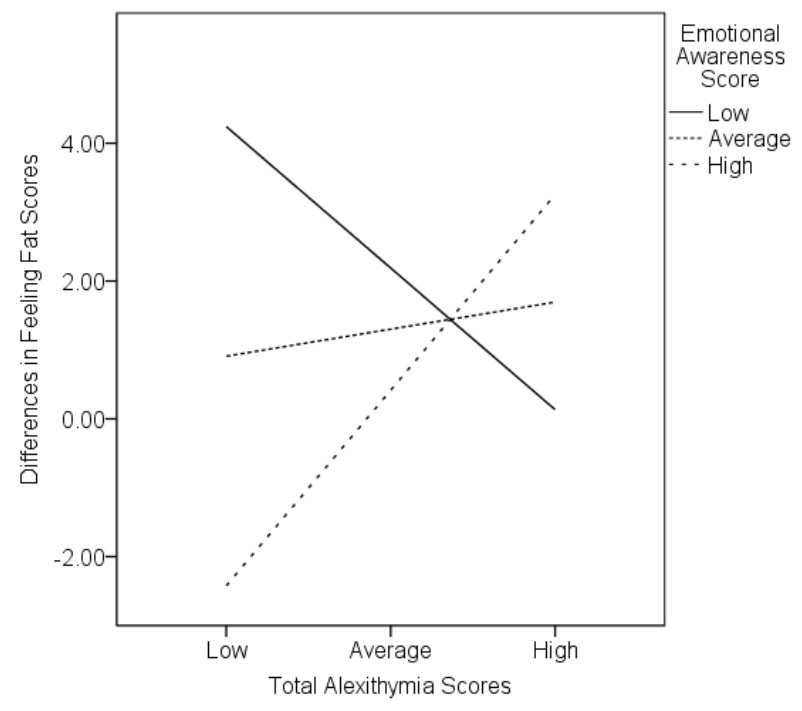

Figure 17. The interactions between total alexithymia and emotional awareness scores for differences in feeling fat scores.

MAIA self-regulation (SR) had a similar pattern to MAIA-EA but the overall model did not reach significance, $F(3,98)=2.658, p=.052$. The greatest increase in feeling fat was found when levels of alexithymia and self-regulation were both low, suggesting that feeling fat is more likely to occur in individuals who have a good understanding of their emotions but do not use their bodily sensations to regulate their distress. At low levels of alexithymia and high levels of self-regulation, individuals are able to successfully understand their emotions and can use their bodily sensations to regulate their emotions. Therefore, they are less likely to feel fat following a distressing manipulation because they can self-regulate. At the opposite end, when high levels of self-regulation are combined with high levels of alexithymia, individuals 
are unable to understand what they are experiencing and therefore cannot utilise their abilities to self-regulate, and in turn experience an increase in feeling fat. However, it should be noted that the differences in feeling fat scores were very small and caution should be taken when interpreting them.

\subsection{Study Five: Final Model}

\subsubsection{The relationship between alexithymia and emotional eating, including interoception, negative urgency and feeling fat}

A serial mediation model was conducted to further explore and extend the theoretical models initially proposed in Study One and Two (Chapter Two) and those in the current study. The first model investigated MAIA-trusting, feeling fat and negative urgency as potential mediators in the relationship between alexithymia and emotional eating (see Figure 18). Total alexithymia scores and the TAS-20 subscales were entered as predictor variables at $X$ with emotional eating as measured by DEBQ as an outcome variable at $Y$. The mediators were entered in the following order: MAIA-T $\left(\mathrm{T} ; M_{1}\right)$, negative urgency $\left(\mathrm{NU} ; M_{2}\right)$, and feeling fat $\left(\mathrm{FF} ; M_{3}\right)$. It was predicted that alexithymia would be indirectly associated with emotional eating via a lack of ability to trust sensations which causes individuals to misinterpret and mislabel their bodily sensations as feelings of fat. In turn, they may and act rashly to alleviate these sensations (negative urgency) through engaging in emotional eating. 


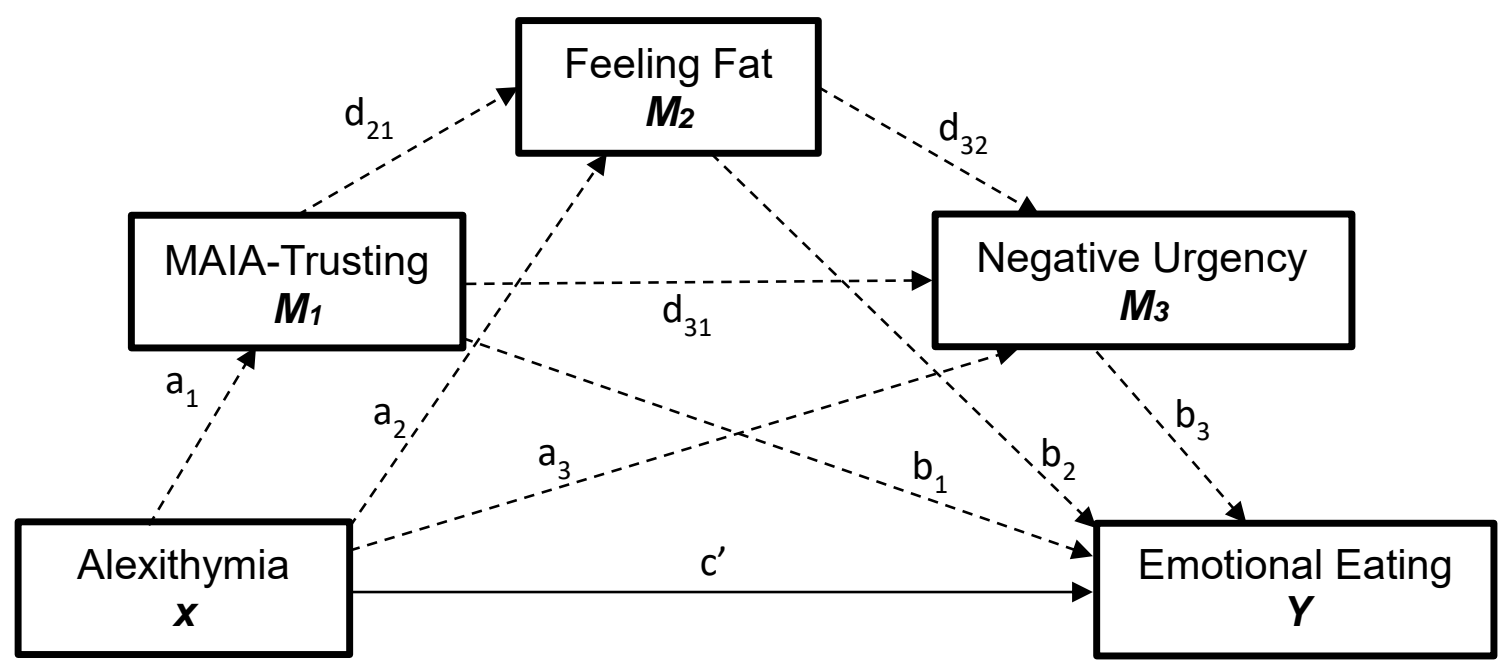

Figure 18. Inclusive model of emotional eating: The proposed direct and indirect relationships between alexithymia and emotional eating including MAIA trusting, feeling fat and negative urgency.

No significant direct effect between total alexithymia score and emotional eating was found. However, three significant indirect effects were found: via NU $\left(a_{3} b_{3}\right), B=.0041, C I=.0011-.0081 ;$ via $\mathrm{T}$ and $\mathrm{FF}\left(a_{1} d_{21} b_{2}\right), B=.0029, C I=.0011-$ .0055 , and via $\mathrm{T}, \mathrm{FF}$ and NU $\left(a_{1} d_{21} d_{32} b_{3}\right), B=.0007, C I=.0002-.0015$. Contrast analysis found no significant difference between the effect via NU and via $\mathrm{T}$ and FF, $B=.0012, C I=-.0029-.0058$, but both of these effects were significantly stronger than the indirect effect via T, FF and NU (via NU: $B=.0034, C I=.0005-.0072$, via $\mathrm{T}$ and FF: $B=.0022, C I=.0004-.0048)$.

When DIF was entered as $X$ (Model 2), a significant direct effect was found $\left(c^{\prime}\right), B=.0230, C I=.0019-.0441, p=.032$. Three significant indirect effects also emerged: (via NU $\left(a_{3} b_{3}\right): B=.0123, C I=.0042-.0231$; via T and FF $\left(a_{1} d_{21} b_{2}\right): B=$ $.0054, C I=.0019-.0104$, and via T, NU and FF $\left(a_{1} d_{21} d_{32} b_{3}\right): B=.0011, C I=.0002-$ .0024). Contrast analysis found that the indirect effect via $\mathrm{T}, \mathrm{NU}$ and FF was significantly weaker than the indirect effects via NU, $B=.0113, C I=.0036-.0215$, and $270 \mid \mathrm{P}$ a g e 
via $\mathrm{T}$ and $\mathrm{FF}, B=.0043, C I=.0009-.0092$. There was no significant difference in the effects via NU and via T and FF, $B=.0069, C I=-.0034-.0189$.

When DDF was entered as $X$ (Model 3), no significant direct effect was found but there were four significant specific indirect effects: via FF $\left(a_{2} d_{21} b_{2}\right), B=.0095, C I$ $=.0023-.0182$; via T and FF $\left(a_{1} d_{21} b_{2}\right), B=.0059, C I=.0020-.0114$; via $\mathrm{FF}$ and NU, $B$ $=.0023, C I=.0004-.0053$, and via T, FF and NU $\left(a_{1} d_{21} d_{32} b_{3}\right), B=.0014, C I=.0004-$ .0031. Contrast analysis found that the indirect effect via FF was not significantly different to the effect via T and FF, $B=.0036, C I=-.0052-.0121$, but was significantly stronger than the indirect effects via FF and NU, $B=.0072, C I=.0007-.0155$, and via T, FF and NU, $B=.0081, C I=.0004-.0172$. The indirect effect via $\mathrm{T}$ and FF was significantly stronger than the effect via T, FF and NU, $B=.0045, C I=.0007-.0097$, but not via FF and NU, $B=.0036, C I=-.0019-.0102$. There was also no significant difference between the indirect effects via FF and NU and via T, FF and NU, $B=$ $.0009, C I=-.0012-.0034$.

Finally, no significant direct effect was found when entering EOT as $X$, (Model 4) but significant specific indirect effects were found via T and FF $\left(a_{1} d_{21} b_{2}\right), B=.0041$, $C I=.0009-.0088$, via $\mathrm{T}$ and NU $\left(a_{1} d_{31} b_{3}\right), B=.0016, C I=.0001-.0043$, and via T, FF and NU $\left(a_{1} d_{21} d_{32} b_{3}\right), B=.0011, C I=.0002-.0026$. There were no significant differences in the strength of effects via $\mathrm{T}$ and $\mathrm{FF}$ and via $\mathrm{FF}$ and $\mathrm{NU}, B=.0025, C I=$ -.0006-.0069, or when comparing indirect effects via FF and NU and via T, FF and NU, $B=.0005, C I=-.0014-.0028$. The indirect effect via $\mathrm{T}$ and FF was significantly stronger than the indirect effect via T, FF and NU, $B=.0030, C I=.0003-.0072$. 


\subsubsection{The relationship between alexithymia and emotional eating including interoception, negative urgency and feeling fat}

The second model included the NA subscale of the PANAS as a measure of negative affect as measured in part one of the study. The model tested the relationship between alexithymia $(X)$ and emotional eating $(Y)$ with negative affect (NA; $\left.M_{1}\right)$, MAIA trusting subscale $\left(\mathrm{T} ; M_{2}\right)$, feeling fat $\left(\mathrm{FF} ; M_{3}\right)$, and negative urgency $\left(\mathrm{NU} ; M_{4}\right)$ as mediators.

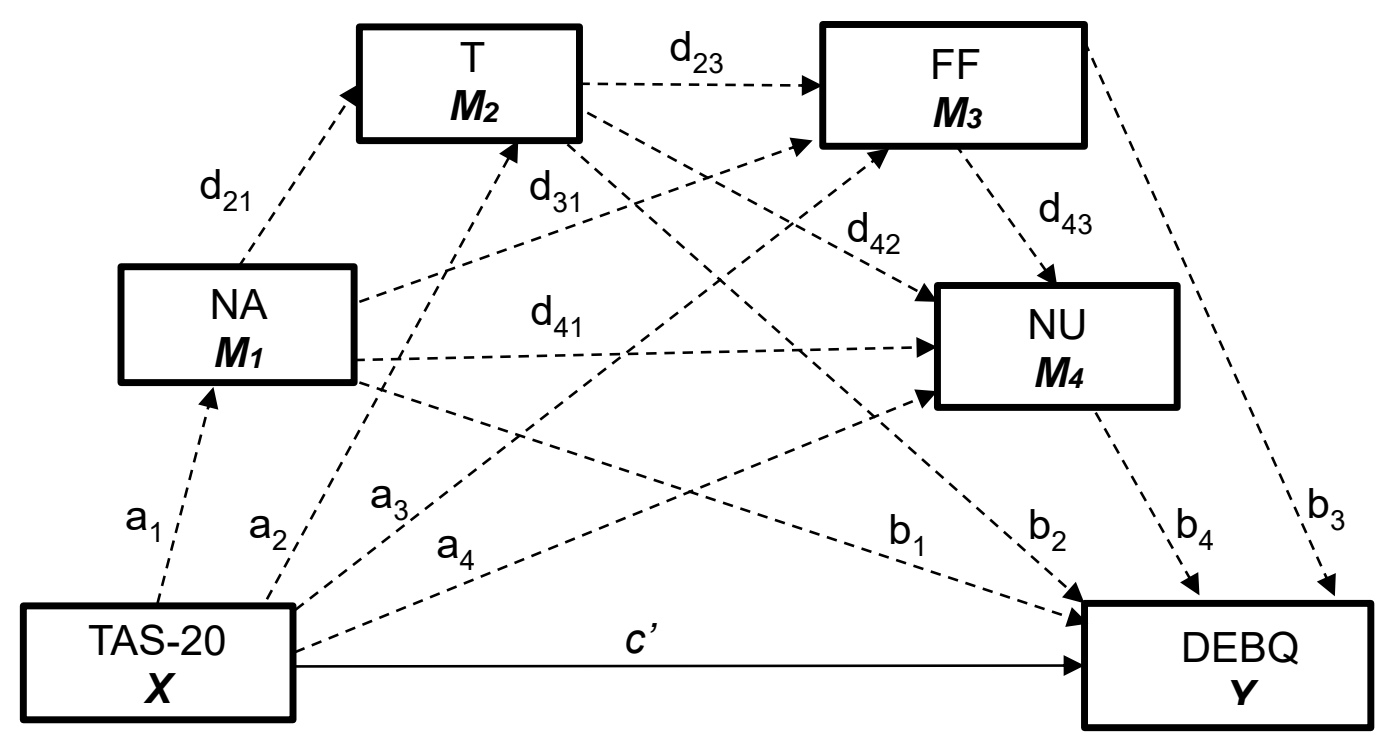

Figure 19. Inclusive model of emotional eating: The proposed direct and indirect relationships between alexithymia (TAS-20) and emotional eating (DEBQ) including negative affect (NA), MAIA trusting (T), feeling fat (FF) and negative urgency (NU) as mediators.

No significant direct effect was found but three significant specific indirect effects emerged: via NU $\left(a_{4} b_{4}\right), B=.0074, C I=.0011-.01969 ;$ via T and FF $\left(a_{2} d_{23} b_{3}\right)$, $B=.0036, C I=.0007-.0076$, and via T, FF and NU $\left(a_{2} d_{23} d_{43} b_{4}\right), B=.0011, C I=.0002-$ 
.0027. Contrast analysis found no significant difference between the indirect effects via NU and via T and FF, $B=.0038, C I=-.0044-.0142$. However, the specific indirect effect via $\mathrm{T}$ and FF was significantly stronger than that indirect effect via trusting, FF and NU, $B=.0063, C I=.0007-.0152$. Finally, there was also no significant difference between the specific indirect effects via $\mathrm{T}$ and $\mathrm{FF}$ and via $\mathrm{T}$, FF and $\mathrm{NU}, B=.0025$, $C I=-.0008-.0066$.

\subsection{Study Five: Discussion}

Study Five aimed to investigate the potential mediating roles of feeling fat and interoceptive awareness in the relationship between alexithymia and emotional eating. Part one consisted of a questionnaire study to explore the relationships between alexithymia, emotional eating, negative urgency, feeling fat and interoceptive awareness. In line with predictions, feeling fat was significantly and positively related to DIF, DDF and total alexithymia scores. Therefore, individuals who are unable to identify and understand their emotions may be more likely to misinterpret negative affect arising from difficult situations as a general sensation of feeling fat. Primarily, it is thought that due to a lack of ability to identify feelings, individuals focus on the

somatic sensations of emotions and hence emotions are physically expressed as experiencing sensations of feeling fat. This is in line with the clinical findings presented by Andersen (2000) who reported that individuals with eating disorders have a tendency to say "I feel fat" rather than focussing on how they feel emotionally. Extending these findings, the results of the current study suggest that such tendencies may also be apparent in individuals without eating disorders and can be further exacerbated if alexithymia is present. 
Similar to Zamariola et al. (2018), a number of significant associations were found between alexithymia and MAIA subscales. Specifically, total alexithymia scores were associated with less awareness of bodily sensations and reduced ability to attend to bodily sensations, link bodily sensations with emotional states, and to regulate distress through the use of one's body. Total alexithymia scores most strongly correlated with trusting scores of the MAIA, suggesting that individuals who experience difficulty regulating their emotions are less likely to feel that they can trust their bodily sensations. In addition, difficulties identifying and describing emotions (DIF, DDF) was associated with greater worry and concern over bodily sensations of pain and discomfort. In addition, and consistent with earlier findings reported in Studies One and Two, significant associations between emotional eating and alexithymia were found, reiterating that when individuals experience difficulty understanding their emotions they may turn to food to alleviate negative emotions as they find them confusing. High levels of emotional eating were also associated with increased worrying and emotional distress due to bodily sensations, greater awareness of the connection between bodily sensations and emotional states, and decreased ability to trust one's bodily sensations. Consistent with previous research (e.g. Young et al. 2017), this suggests that individuals with poorer interoceptive ability experience a greater tendency to emotional eat, presumably because they misinterpret bodily sensations. For example, when experiencing increased levels of anxiety, a common accompanying bodily sensation is the feeling of butterflies in the stomach. Individuals who have a tendency to misinterpret bodily sensations may interpret butterflies in the stomach as feelings of hunger.

Greater sensations of feeling fat were also significantly and positively related to higher levels of self-reported emotional eating. Although this may appear 
counterintuitive, individuals who experience the sensation of feeling fat and are prone to engaging in emotional eating, may do so as they have learnt that consumption of food will make them feel better. That is, they may believe that overconsuming food does not matter and has no negative consequences as they are already feeling fat. However, as there is scant research exploring why individuals may engage in emotional eating when feeling fat, further research is needed to investigate this proposal. Indeed, it is also possible that individuals will not actually engage in emotional eating in response to feeling fat. As has been highlighted previously in this thesis, self-reported emotional eating has not been consistently related to food intake following an emotional experience (see Chapter Four). Therefore, individuals may experience the sensation of feeling fat and believe they engage in emotional eating in response but may not actually go on to increase their food intake. To explore this proposal, food consumption could be explored following an episode of feeling fat, as well as a qualitative study performed to investigate how individuals respond to feeling fat generally. Finally, feeling fat was associated with reduced ability to self-regulate distressing emotions, being less trusting of one's body, increased attention and awareness of bodily sensations, as well as reduced ability to not worry about sensations. Overall, these findings suggest that feeling fat is an important consideration in understanding the mechanisms underpinning emotional eating and may also help increase the understanding of how interoceptive awareness may also play a role.

In contrast to previous research (e.g. Cooper et al., 2007), BMI scores were not significantly correlated with feeling fat, which could be partly explained by the adoption of varying measures and different samples across studies. Here, the study was conducted on a representative sample of healthy females recruited from Swansea university and through social media advertisements, whereas Cooper et al. (2007) 
recruited a greater diversity of females (i.e. females with AN and females who were and were not dieting), although it should be noted that no measure of dieting was recorded in the curry study. Consequently, even though average BMI in the current study was very similar to the average BMI of the dieting group in Cooper et al. (2007) lower BMI scores were reported for both the AN and non-dieting groups. It may be that feeling fat is associated with low BMI in individuals who are dieting or present with eating disorders, as when they experience sensations of feeling fat they look to reduce these feelings by restricting food intake and via other mechanisms (i.e. excessive exercising). In contrast, individuals without eating disorders may experience feeling fat because of a higher BMI, or as found in the current study, have an increased tendency to emotional eat which may in turn increase their BMI in the longer term. The current study also used the feeling fat subscale of the BAQ, whereas Cooper et al. (2007) used a semi-structured interview (without established psychometric properties) where participants were asked to recall their first and most recent memories of feeling fat. As there is no other research on the association between feeling fat and BMI, further research utilising robust methods is needed.

To conclude part one of the study, serial mediation models were performed to explore whether interoception and feeling fat mediates the relationship between alexithymia and emotional eating. In line with predictions, interoception, and specifically the trusting subscale of the MAIA, and feeling fat significantly mediated the relationship. This finding was significant for alexithymia total and subscale scores, suggesting that increased levels of alexithymia may lead to difficulties being able to trust one's bodily sensations, increased feelings of fat, and in turn, emotional eating. Replicating and expanding the results of Study Four, there were also significant direct effects of DIF, DDF and total alexithymia scores on emotional eating. In contrast, the 
self-regulation and noticing subscales of the MAIA were not significant mediating factors. However, the mediation pathway through self-regulation and feeling fat approached significance; the lower CI was 0 and therefore was not significant as the CI contained 0 (Hayes, 2013). This suggests that greater levels of alexithymia lead to a reduced ability to regulate distress using one's bodily sensations which increases the likelihood of feeling fat and engaging in emotional eating. Awareness of bodily sensations (MAIA-noticing) was also significantly positively correlated with feeling fat and negatively related to alexithymia. However, within the serial mediation pathway, awareness of bodily sensations and feeling fat did not significantly mediate the relationship between alexithymia and emotional eating.

The second part of the study aimed to explore whether feeling fat could be manipulated within a laboratory setting. Unfortunately, the social comparison manipulation used to induce feelings of fatness was unsuccessful. Similar ratings of feeling fat, awareness of bodily sensations and attractiveness were reported postmanipulation across the four groups (Neg-FF, Pos-FF, Neut. and Neg-Gen). Exploring the comments participants made during the manipulation task shines some light on why the manipulation may have been unsuccessful. Although the majority of participants in the positive feeling fat group commented on feeling good about themselves, several also reported they would feel bad, awkward or guilty that their friends did not feel as good about themselves and that attention was focussed on them. In addition, several participants in the positive feeling fat group commented on the amount of exercise they undertook, justifying their beliefs as a result of working hard. Therefore, this may help to explain why a reduction in feeling fat scores were observed. In terms of the negative feeling fat group, participants often remarked the comment was "rude" but justified their food choice as eating out was a treat, stating 
that they should eat what they like. The justification of eating 'unhealthy' foods as a treat may have resulted in the small increase in feeling fat scores found. Future studies need to consider these influences in manipulating sensations of feeling fat. It may be that levels of exercise and the participants views on healthy and unhealthy foods need to be recorded so they can be controlled for. It is also worth considering different scenarios for the task to avoid participants viewing intake in these circumstances as a "treat". In addition, it may have been difficult for an individual to think about such a situation. For example, they may have never experienced a similar situation and therefore were unable to immerse themselves into the thoughts and feelings expected to be generated. The development of a future feeling fat manipulation should be based upon the situations, thoughts and feelings experienced by individuals who regular experience the sensation of feeling fat. Such information could be gathered in a qualitative study in order to develop the manipulation. Alternatively, EMA could be used to monitor natural changes in feeling fat and the changes following such sensations.

Moderation analysis was also conducted to examine whether participants more susceptible to the feeling fat manipulation could be predicted, regardless of group. Attention regulation and emotional awareness significantly moderated the relationship between alexithymia and differences in feeling fat scores. At low levels of alexithymia, similar changes in feeling fat scores were experienced. When individuals experience difficulty identifying their bodily sensations combined with good attention to them, they were more likely to report feeling fat. Similarly, increased feelings of fat were reported when individuals had a good understanding of their emotions but experienced difficulty connecting bodily sensations with emotions. 
In the final model, the DIF subscale was found to directly influence emotional eating, reinforcing the argument that when an individual has difficulty identifying emotions they may be more likely to engage in emotional eating. These findings extend the results found in Studies One and Four, replicating the direct relationship between alexithymia and emotional eating when captured by a different measure of emotional eating (i.e. the DEBQ). It was proposed that increases in alexithymia would decrease an individual's ability to trust their bodily sensations, and in turn, increase feelings of fat which they would want to alleviate as quickly as possible (negative urgency) and emotionally eat to do so. Although this specific indirect effect was significant for the total and subscale scores of alexithymia, it was not the strongest effect. The strongest specific indirect effects were via negative urgency only and via a lack of ability to trust one's bodily sensations and feeling fat.

The current research is not without its limitations. First, the sample consisted of only females and whilst the use of this sample is common in eating behaviour research, the results cannot be generalised to male samples. It is predicted that due to higher levels of alexithymia in males, they may experience a greater tendency to feel fat. However, it is possible that this would not necessarily result in increased engagement in emotional eating. Instead, it is possible that males would engage in different maladaptive coping behaviours to regulate these feelings, such as the use of alcohol (Cooper, Russell, Skinner, Frone, \& Mudar, 1992) or excessive exercise due to men not wanting to 'feel weak'. Although this is only a theory and there is currently no research exploring how the experience of feeling fat may differ across genders. A second limitation is that food intake was not measured. Therefore, as the current study did not examine food intake, it remains unknown whether the relationship between feeling fat and emotional eating can be extended to actual eating behaviour. To test 
this, a successful manipulation procedure needs to be designed followed by a bogus taste test. This would also require a suitable control group similar to the current study to ensure participants responded to an increase in feeling fat rather than an increase in negative emotion.

Third, whilst the NA subscale of the PANAS provides a measure of negative affect, it does not capture depression and anxiety symptoms as measured by the BDI and BAI in Studies One, Two and Four. As a result, the models tested in the current study are not a true extension of those proposed and tested in previous chapters of this thesis. As a consequence, it was not possible to distinguish individual aspects of negative affect from a broader measure which reduces the ability to test the final model with depression and anxiety separately as the previous studies have done. Future studies should ensure the inclusion of BDI and BAI as part of the measures collected to allow for a full examination of the models and allow them to be advanced and the role of depression and anxiety to be explored appropriately.

\subsection{Study Five: Conclusion}

Overall, the results from Study Five are consistent with previous studies reporting higher levels of emotional eating in individuals with poor interoceptive awareness and alexithymia (Larsen et al., 2006; Ouwens et al., 2009; Spoor et al., 2007; van Strien \& Ouwens, 2007). The under researched concept of feeling fat was also explored, finding significant associations with alexithymia, emotional eating and interoceptive awareness. Specifically, feeling fat was related to difficulty identifying and describing emotions, an increased awareness of bodily sensations and a reduced ability to notice, self-regulate and trust bodily sensations. Additional mediating factors 
(MAIA-T and FF) were included to further expand the models developed in Chapters Two and Four. Being able to trust one's bodily sensations and feeling fat significantly mediated the relationships between alexithymia and emotional eating. A final model inclusive of negative affect and negative urgency was also tested, where the strongest indirect effects occurred were found via negative urgency alone and via trusting and feeling fat.

\subsection{Chapter Five: Key Findings}

- Significant associations were found between feeling fat, emotional eating, alexithymia, trusting bodily sensations and negative urgency.

- Total alexithymia scores and difficulty identifying feelings directly predicted emotional eating scores as measured by the DEBQ.

- Increased total and subscale scores of alexithymia indirectly predict emotionally eating through reduced interoceptive awareness and increases in feeling fat. This was the strongest indirect effect.

- The manipulation of feeling fat through social comparison paradigms did not lead to changes in feeling fat, being aware of bodily sensations or feelings of attractiveness.

- Attention regulation and emotional awareness moderated the relationship between total alexithymia scores and feeling fat. 


\section{Chapter Six: Discussion and Conclusion}

\subsection{Original aims and objectives}

The overarching aim of this thesis was to elucidate the mechanisms that underpin emotional eating. Specifically, the aim was to explore the role of emotional dysregulation, as conceptualised by alexithymia, in understanding emotional eating. Alongside emotional dysregulation, negative affect, impulsivity, interoceptive awareness and feeling fat were considered as key factors that may influence the relationship between alexithymia and emotional eating. Previous studies have begun to examine these factors individually (e.g. Fink et al., 2010; Ouwens, van Strien, \& van Leeuwe, 2009; Ouwens, van Strien, van Leeuwe, \& van der Staak, 2009; Pike, 2013; van Strien et al., 2005) but this thesis is the first to comprehensively explore all factors in one inclusive model. In order to develop this model, five studies have been presented which aimed to:

1. Use alexithymia as a correlate of emotional dysregulation to explore its role in emotional eating.

2. Examine the key contributing factors that influence the relationship between emotional dysregulation and emotional eating, and in turn BMI.

3. Test the theoretical model of emotional eating, as originally presented in Figure 1, Chapter 1 (see Figure 20).

4. Investigate whether simulating emotional dysregulation in an experimental setting directly affects food intake.

5. Extend the theoretical model to account for interoceptive awareness and feeling fat. 


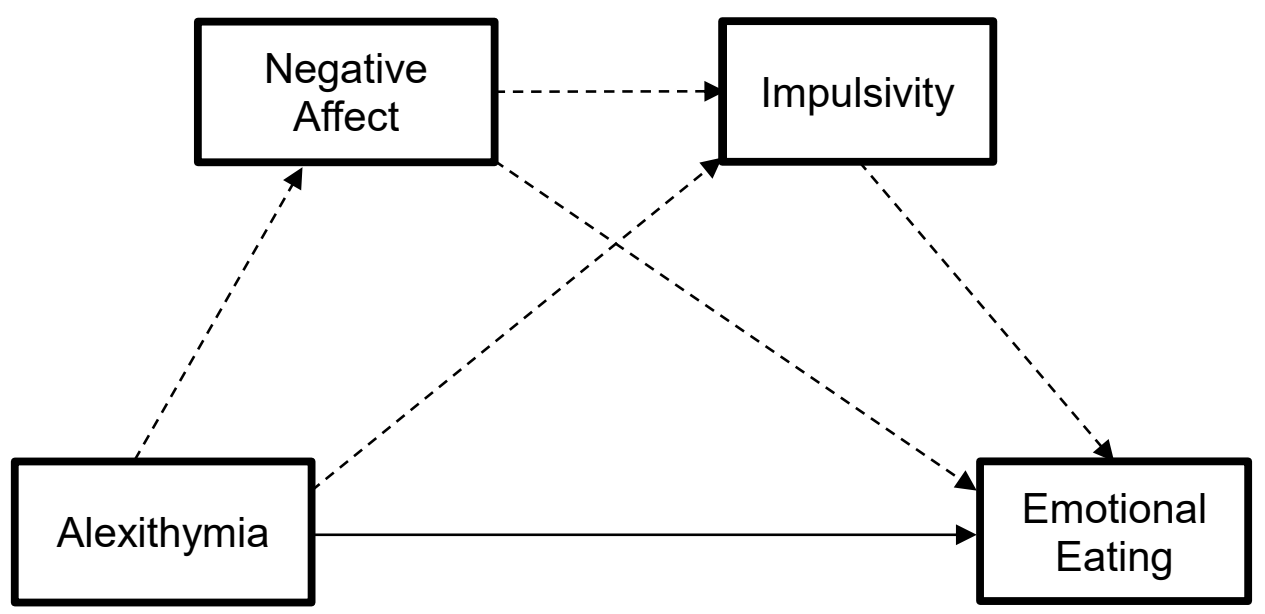

Figure 20. Figure 1 as presented in Chapter One. Inclusive model of emotional eating: The proposed direct and indirect relationships between alexithymia and emotional eating, including negative affect and impulsivity as mediators.

\subsection{Summary of findings}

\section{Aim 1: To use alexithymia as a correlate of emotional dysregulation to explore its role in emotional eating}

Consistently throughout this thesis, emotional eating was significantly and positively associated with alexithymia (evidence from studies in Chapters Two and Five). Broadly, total alexithymia, as well as the difficulty identifying and describing feelings subscales, were related to emotional eating as measured by the EES, TFEQ and DEBQ. This indicates that greater difficulties identifying and describing emotions were significantly related to a greater level of self-reported emotional eating. Individuals who are unable to correctly recognise what emotions they are experiencing and sufficiently express them to others may misinterpret the experience of certain emotions as feelings of hunger and turn to food in response. Therefore, the use of alexithymia to explore the role of emotional dysregulation in emotional eating is 
supported. However, consistent results were not found across measures of emotional eating and subscales of the TAS-20. For example, in Study One (Chapter Two) total alexithymia scores and DIF were significantly related to emotional eating as measured by the EES but were not related to TFEQ scores. Therefore, whilst it appears that alexithymia and emotional eating are related overall, when examining the relationship between specific factors of alexithymia or the use of different measures of emotional eating, caution is needed when interpreting results. Additionally, this suggests that other factors may also play a role in the relationship.

\section{Aim 2: To examine the key contributing factors that influence the relationship between emotional dysregulation and emotional eating, and in turn BMI}

Negative affect (as defined by depression and anxiety) and negative urgency (a facet of impulsivity) were identified as potential key contributing factors. Negative urgency was significantly and positively associated with emotional eating and was also related to BMI (Study Two, Chapter Two only). Depression and anxiety also had significant positive associations with emotional eating but not BMI. Therefore, increased experience of depressive and anxiety symptoms and a desire to alleviate feelings of negative affect are associated with a greater tendency to emotionally eat. This supports the inclusion of negative affect and negative urgency as mediators in the model examining the relationship between alexithymia and emotional eating. As with alexithymia, the measure of emotional eating used resulted in different significant associations with negative affect. For example, emotional eating as measured by the TFEQ was significantly associated with depression but not anxiety. This further supports examining the role of depression and anxiety separately. 


\section{Aim 3: To test the theoretical model of emotional eating, inclusive of alexithymia, negative affect and negative urgency}

The third aim was to test the direct and indirect effect of alexithymia on emotional eating, including negative affect (separate models for depression and anxiety) and negative urgency as mediators. The following key findings emerged:

1. Significant indirect effect via negative affect and negative urgency in Study One (Chapter Two)

2. Significant indirect effect via negative affect alone in Study Two (Chapter Two)

3. Significant direct effect of total alexithymia scores and the DDF and EOT subscales on emotional eating in Study Four (Chapter Four)

These findings show that emotional dysregulation does play a role in emotional eating, whilst inconsistencies across studies also highlight that negative affect and negative urgency are unable to fully explain the relationship. Tentative conclusions can be drawn for the role of emotional dysregulation, negative affect and negative urgency in understanding the mechanisms of emotional eating, but the specific pathways remain unclear.

The next logical step was to extend the model in order to test whether it could predict food intake. No significant direct or indirect effect of alexithymia on emotional eating where found (Study Four, Chapter Four). This highlights that there is a difference between an individual's perception of their eating behaviour in response to emotions and their actual behaviour. Further investigation is required to examine these differences and explore why individuals believe themselves to be emotional eaters when they do not increase their intake of food. This relates back to the idea of a "triple recall bias" first presented in section 1.3.1. To be able to accurately rate to what extent 
they emotional eat, individuals need to recall when they experienced negative emotions, their eating behaviour in response to the emotions and then successful associate the two. The findings of Study Four (Chapter Two) would suggest that individuals find it difficult to accurately recall how they respond to negative emotions with regards to their eating behaviours.

\section{Aim 4: To investigate whether simulating emotional dysregulation in an experimental setting directly affects food intake}

The fourth aim was to test the hypothesis that there is a direct influence of ambiguous mood on food intake. Evidence suggests alexithymia is associated with BMI, so this was an attempt to test the idea that the association was due to overeating. A key feature of alexithymia is confusion or uncertainty about emotions, and therefore, the induction of an emotionally confusing mood state was used as an analogue of alexithymia. In order to induce emotional confusion, a novel mood induction paradigm was developed and tested prior to exploring its effect on food intake (Study Three, Chapter Three). Study Four (Chapter Four) explored the effect of induced positive, negative and emotionally confusing mood states on food intake. Each participant completed a control condition and one of three experimental mood inductions a week apart. There was no significant difference in the amount of food (in grams) consumed in the control versus experimental condition for any group (positive, negative and emotional confusion). 


\section{Aim 5: To extend the theoretical model to account for interoceptive awareness and feeling fat}

Finally, the theoretical model was extended to include interoceptive awareness and feeling fat as mediating factors in the relationship between alexithymia and emotional eating because negative affect and negative urgency were unable to fully mediate and explain the relationship. The results of Study Five (Chapter Five) found that alexithymia was directly associated with emotional eating. An additional indirect effect via trusting one's bodily sensations and feeling fat was also found. Similar significant results were found for total alexithymia scores and its subscales which suggests that all facets of alexithymia play a role. Increased levels of alexithymia may lead to an increased likelihood that individuals will be unable to trust their bodily sensations and experience feeling fat which may result in a greater propensity to emotionally eat. This led to a final model where trusting, feeling fat and negative urgency were entered in a serial mediation model. This analysis revealed multiple significant indirect effects via:

1. negative urgency only

2. trusting and feeling fat

3. trusting, feeling fat, and negative urgency

4. feeling fat only

The strongest indirect effects were through trusting and feeling fat and through negative urgency alone. Therefore, increased levels of alexithymia may result in a reduced ability to trust one's bodily sensations which may lead to a greater experience of feeling fat. In order to alleviate these sensations, individual's act impulsively (negative urgency) through emotionally eating. Both these extended models highlight 
that emotional dysregulation is a central part of emotional eating, but further emphasises the complicated nature of emotional eating.

\subsection{Implications of findings}

Understanding the mechanisms underpinning emotional eating is important due to its associations with BMI and the related negative health consequences. Emotional eating is one of many contributing factors leading to a rise in overweight and obesity. However, given the financial and societal costs of the obesity epidemic, any possible approach to reduce obesity is welcomed. Therefore, exploring the role of emotional eating in overconsumption is critical, as understanding the fundamental influences on the eating behaviour may help develop interventions. Emotional eating is also prevalent in eating disorders, in particular AN binge eating/purging type and binge eating in BN (Ricca et al., 2012) and BED (Pinaquy et al., 2003), although this is outside the remit of this thesis. If emotional eating is a form of regulating emotions, it is important to understand that in order to support the intention to manage one's weight, there is a need to also address emotional eating. In support of this, Braden et al. (2016) found that a behavioural weight loss programme decreased emotional eating and improved weight loss. They argued that reducing an individual's tendency to emotionally eat is vital in achieving weight loss. Consequently, combining weight loss programmes with skills training to decrease emotional eating may be key. One possible intervention to target emotional eating is through Dialectical Behaviour Therapy (DBT; Linehan, 1993a; 1993b) and initial findings in eating disorders have shown positive results (e.g. Roosen et al., 2012; Safer, Robinson, \& Jo, 2010; Safer, Telch, \& Agras, 2001; Telch, Agras, \& Linehan, 2000, 2001). Telch et al. (2001) found that 
following DBT adapted for the use in BED, individuals reported significant improvements on measures of binge eating and eating pathology. The DBT in this study targeted emotional regulation, mindfulness and distress tolerance skills. Additionally, in a preliminary study, Clyne and Blampied (2004) found that five (out of 11) participants reduced their levels of binge eating to a non-clinical level and reported small improvements in alexithymia following emotion recognition and regulation training. Alongside DBT, mindfulness has also been shown to effectively reduce levels of binge eating and emotional eating (for reviews see: Katterman, Kleinman, Hood, Nackers, \& Corsica, 2014; O’Reilly, Cook, Spruijt-Metz, \& Black, 2014). Mindfulness training enables individuals to develop skills to help them attend to their negative emotions and accept them instead of reacting impulsively to them (e.g. suppressing them, masking them; O'Reilly et al., 2014). However, sample sizes in these studies remain small and very few have comparison conditions. Consequently, caution needs to be taken when drawing conclusions until larger randomised controlled trials are conducted. Furthermore, in a review of the literature, Katterman et al. (2014) found that despite reductions in binge eating and emotional eating, mindfulness did not result in weight loss.

Tapper and collegues (2009) developed a programme specifically for weight loss through adapting a mindfulness-based technique. Their intervention included concepts, exercises and metaphors adapted for weight loss from Acceptance and Commitment Therapy (e.g. Hayes \& Smith, 2005), which participants completed alongside their usual diet/weight loss programme. This was compared to a control condition where participants continued with their usual diet/weight loss programme. They found no significant difference in weight loss (BMI change) but a significant difference in physical activity levels between an intervention and control condition at 
six months. However, change in BMI was significantly related to how often participants in the intervention condition reported using the workshop principles at six months. Furthermore, when excluding participants who reported never having used the intervention at six months, significant differences in BMI and physical activity emerged. Specifically, a greater reduction in BMI scores and increased levels of physical activity were found in the intervention condition. Although not designed to specifically target emotional eating, one of the key intervention components was to help participants to tolerate negative feelings. However, there was no significant reduction in emotional eating specifically, but a significant reduction in binge eating was seen and change in BMI was associated with emotional eating. Consistent with this, Kearney et al. (2012) found that a mindfulness based stress reduction intervention did not reduce levels of emotional or uncontrolled eating. Despite the increasing number of studies exploring interventions, there continues to be a lack of research examining treatment methods against one another as found in a review of emotional eating and weight loss/management (Frayn \& Knäuper, 2017). This also includes a lack of development of combined treatments (e.g. cognitive behavioural therapy and mindfulness; Corsica, Hood, Katterman, Kleinman, \& Ivan, 2014; Goldbacher et al., 2016). Frayn and Knäuper (2017) concluded their review by reiterating the need for large scale randomised control trials and the development of interventions which help individuals who emotionally eat to be able to effectively and efficiently target weight loss.

As highlighted throughout this thesis, the factors that influence emotional eating are complex and this may be why the development of interventions has been difficult. It may be that interventions need to also address trust in bodily sensations (interoceptive awareness) and reduce the tendency to react rashly (negative urgency). 
Currently it does not appear that interventions specifically targeting negative urgency are available as there are scant reports in the literature, but the need for such interventions is often highlighted as an important next step (e.g. Deckman \& DeWall, 2011; Kaiser, Milich, Lynam, \& Charnigo, 2012; Karyadi \& King, 2011). Although, strategies to attenuate the intensity of the negative emotions may in turn reduce how likely an individual is to react rashly in response to such emotions (Cyders \& Smith, 2010).

In relation to interoceptive awareness, there have been inconsistent findings concerning whether improvements can be made to an individual's ability to accurately perceive and interpret bodily sensations. Some studies have shown no significant changes in interoceptive awareness following mindfulness-based interventions (Melloni et al., 2013; Parkin et al., 2014) and other relaxation techniques (Fairclough \& Goodwin, 2007; Khalsa et al., 2008; Stevens et al., 2011). Although, studies that use self-focused attention (e.g. staring at a mirror or a photograph of oneself) have found significant improvements in the accuracy of heart beat perception tasks (Ainley, Maister, Brokfeld, Farmer, \& Tsakiris, 2013; Ainley, Tajadura-Jiménez, Fotopoulou, \& Tsakiris, 2012; Maister \& Tsakiris, 2014). However, Study Five (Chapter Five) found that it was specifically trusting one's bodily sensations that was a significant mediator, and therefore, interventions that are more focused on trusting bodily sensations may be more applicable. Research investigating such interventions is relatively new and limited, but positive outcomes have been found. For example, Fissler et al. (2016) found that following mindfulness training (i.e. guided meditation, body scanning and mindful moving) individuals with depression reported a greater ability to trust their bodily sensations in comparisons to a control group (scheduled rest with music). Furthermore, in a mindfulness meditation programme to reduce 
burnout in physiotherapists and sports therapists, Mohammed and colleagues (2018) found significantly higher scores on the MAIA trusting subscale, indicating a greater ability to trust bodily sensations following the programme. Further, they found the greatest improvement was following face-to-face group meditation in comparison to a self-directed group. Additional studies have also found yoga/meditation (Tolbaños, Miró, Ibáñez, \& Betancort, 2017) and guided walking (Teng, Yeh, \& Wang, 2018) to be effective interventions in improving trust in one's bodily sensations. These studies highlight that improvements can be made in interoceptive awareness and in particular, the ability to trust one's bodily sensations. Therefore, targeting these skills may help aid the development of interventions of emotional eating.

This thesis also highlights that depression and anxiety should be examined separately in the context of emotional eating as different effects emerged when entering depression or anxiety into the developed model. For example, in Study Two (Chapter Two) alexithymia had a significant indirect effect on BMI via depression only when BDI scores were included in the model, but when BAI scores were included, the indirect effect was via anxiety and negative urgency.

Overall, this thesis provides support for the role of emotional dysregulation in emotional eating. The idea that individuals who are unable to process their emotions appropriately and instead use emotional eating as a coping mechanism has been conceptualised by the term "swallowing their emotions" (Fox et al., 2017) and as captured in the title of this thesis "consuming your feelings". This highlights that interventions developed which target improving an individual's ability to regulate their emotions may be useful. DBT and mindfulness have currently been identified as possible interventions, but they require further robust testing before firm conclusions 
can be drawn. Interventions that also improve an individual's ability to trust one's bodily sensations are also important.

\subsection{Strengths and Limitations}

\subsubsection{Strengths}

A clear strength of this thesis is that it offers novel insight into the relationship between emotional dysregulation and emotional eating. For the first time, an inclusive theoretical model incorporating multiple mediating factors (negative affect, negative urgency, interoceptive awareness and feeling fat) was developed. The mechanisms underpinning emotional eating are complex and this thesis takes the first steps in extending the available knowledge of the relationships between factors and emotional eating and establishing these within one model. Previously, Ouwens et al. (2009) developed a model exploring the mediating role of impulsivity and DIF in the relationship between depression and emotional eating. Original contributions have been made through looking at alexithymia as a whole but also at its three facets (DIF, DDF, and EOT) and how they interplay with negative affect, negative urgency and interoceptive awareness (Studies One and Two, Chapter Two). In addition, the model was extended to include actual food intake (Study Four, Chapter Four) and the model was further developed to include interoceptive awareness and feeling fat (Study Five, Chapter Five).

Secondly, this thesis tested the proposed theoretical model through robust and rigorous self-replication testing (Studies One, Two, Four and Five). The core element of the model was to test the direct and indirect effects of alexithymia on emotional 
eating. This model was then tested in a number of ways. Firstly, exploratory analysis was conducted in a student sample using the EES and TFEQ as measures of emotional eating followed by a self-replication in a more representative general population sample. The next step extended the model to include food intake and also test the original model again in a student and staff sample from Swansea University. Finally, the model was advanced to incorporate interoceptive awareness and feeling fat and used the DEBQ measure of emotional eating. Although results from each study differed slightly, overall, they confirm a role of emotional dysregulation in emotional eating. The contrasting findings confirm that models need to be thoroughly examined during their development and need to be interpreted with caution until they can be robustly tested.

Thirdly, the studies were carried out on participants from a non-clinical population. This decision was made to reduce the number of confounding factors that accompany eating disorders. For example, eating disorders are highly comorbid with psychopathology (Hudson, Hiripi, Pope, Kessler, \& Kessler, 2007; Swanson, Crow, Grange, Swendsen, \& Merikangas, 2011). Furthermore, and as previously mentioned, emotional eating is one potential driver to overconsumption and in turn increased BMI. Therefore, emotional eating is not just an eating behaviour important in eating disorders but is also applicable to the wider population.

Fourthly, the initial first steps in creating an emotionally confusing mood manipulation as an analogue for alexithymia is a strength for the development of mood induction procedures. This was based on the idea that alexithymia is in part about being confused and unsure about feelings. The development of a mood induction to elicit an emotionally confusing mood state was used to empirically explore differences in food intake. Study Three presented both qualitative and quantitative findings suggesting 
that the manipulation of emotionally confusing emotions is significantly different to negative emotions. Consequently, inducing emotional confusion could be used as an analogue of alexithymia. However, these findings were not replicated in Study Four highlighting this paradigm still requires further development. It is difficult to imitate emotional confusion in the laboratory and it is likely that alexithymia is also more complicated. For example, as mentioned in section 4.5. mixed emotion/emotionally confusing events are harder to recall and there are other aspects of alexithymia that are not captured simply by examining emotional confusion (e.g. elements of EOT).

\subsubsection{Limitations}

Although it is the most reliable and valid method for measuring several characteristics of alexithymia (Kirmayer \& Robbins, 1993; Taylor \& Bagby, 1988; Taylor, Bagby, \& Parker, 1991), a possible weakness of the TAS-20 is that it may focus more on the cognitive deficits and less on the emotional component of alexithymia (Larsen, Brand, Bermond, \& Hijman, 2003). Indeed, Larsen and colleagues (2003) also argue that the majority of items in the TAS-20 focus on the cognitive elements of the personality trait, forgetting the emotional aspects. It can be argued that the scale, whilst psychometrically sound (Bagby, Parker, et al., 1994; Bagby, Taylor, et al., 1994; Parker et al., 2003; Taylor, Bagby, \& Parker, 2003), is flawed by the concept that alexithymia is characterised by a reduced ability to understand emotions and the scale asks individuals to rate themselves on their ability. Therefore, it is possible that individuals with alexithymia have difficulties in making accurate judgements (Lane, et al., 1996). Specifically, how far can someone reflect on 
their own emotional perception when it is self-evaluated (Lundh, Johnsson, Sundqvist, \& Olsson, 2002).

In addition, if individuals have difficulty making judgements of their ability to understand emotions, then it is conceivable that this may also translate to difficulties in identifying how they respond to these emotions. In particular, identifying whether they engage in emotional eating or not. However, studies have shown good concordance across self-report and proxy ratings of alexithymia (Sylvie Berthoz, Haviland, Riggs, Perdereau, \& Bungener, 2005; Lumley, Gustavson, Partridge, \& Labouvie-Vief, 2005), and in particular, Berthoz and colleagues (2007) found that the OAS (Obeserver Alexithymia Scale; Haviland, Louise Warren, \& Riggs, 2000), TAS20 and BVAQ-B (Zech, Luminet, Rimé, \& Wagner, 1999) were all significantly and moderately correlated amongst women with eating disorders. These results highlight that individuals are able to detect and report on their ability to understand emotions and are in line with how others perceive their ability. Furthermore, it could be argued that a proxy rating would be unable to comment on an individual's level of alexithymia as it is a personal experience in which not all features can be observed outwardly. For example, the aspects of alexithymia related to limited imagination. Moreover, Meganck and colleagues (2010) argued that the OAS was a measure of the consequences of alexithymia rather than the core characteristics (i.e. difficulty identifying and describing emotion and externally oriented thinking).

A second limitation is the ratios of males and females. The majority of eating behaviour research is conducted in samples of females and this means results are unable to be generalised to male populations. As previously mentioned, males have increased levels of alexithymia (e.g. Honkalampi, Hintikka, Tanskanen, Lehtonen, \& Viinamäki, 2000; Mattila, Salminen, Nummi, \& Joukamaa, 2006; Parker, Taylor, \& 
Bagby, 2001), and are less likely to report experiencing depressive symptoms (Piccinelli \& Wilkinson, 2000; Van de Velde et al., 2010) and emotional eating (Larsen et al., 2006). Therefore, it is unlikely that the models developed will be applicable to males as they will have been tested with lower overall levels alexithymia and depressive symptoms and higher levels of emotional eating. It is important to develop models that have equal representation of males and females or develop models within each sex independently. Eating disorders in males are increasing (e.g. Kjelsås, Bjørnstrøm, \& Götestam, 2004; Smink, Van Hoeken, \& Hoek, 2012), highlighting that they are not just prevalent in females. In addition, due to differences in the development of eating disorders across males and females, such as weight histories, compensatory behaviours and muscle dysmorphia (for review, see Strother, Lemberg, Stanford, \& Turberville, 2012), it may be worth exploring the mechanisms underpinning emotional eating in males and females separately.

Thirdly, the studies presented in the current thesis are cross-sectional and causal relationships cannot be derived. For example, the relationship between emotional eating and BMI could be interpreted as either emotional eating causes increased BMI or individuals who have a high BMI are more likely to turn to emotional eating. Put simply, it remains unclear whether obesity is a cause or consequence of emotional eating, and it is likely that the two go hand in hand together. Despite the cross-sectional nature of this study being unable to draw more definitive conclusions, it does provide a platform for future empirical questions. For instance, can a similar model be developed and applied to eating in response to positive emotions? Do interventions that target a particular factor in the model reduce emotional eating, and in turn BMI? Why is the sensation of feeling fat associated with increases in emotional eating? Longitudinal studies are required to be able to help further deconstruct the 
associations. Such studies would also additionally test the theoretical models. They could be advanced to predict changes in weight gain and explore how emotional eating may fluctuate over time as a consequence to changes in negative affect. Qualitative studies are also best suited to address the relationship between feeling fat and emotional eating. As it is counterintuitive for these factors to be related, exploring the perspectives of individuals who experience feeling fat and emotionally eat will help further our understanding.

\subsection{Wider implications and future directions}

\subsubsection{Wider implications}

Given that the consumption of food is a form of emotion regulation, it seems plausible that the results of this thesis may be generalisable to other behaviours. Existing literature suggests that alcohol, substance use and compulsive buying are other behaviours that may also be employed as emotion regulation strategies (e.g. Cooper et al., 1992; Fenton-O’Creevy, Dibb, \& Furnham, 2018; Gaher, Arens, \& Shishido, 2015; Karyadi \& King, 2011; Kober, 2014; Menary et al., 2015; Petit et al., 2015; Pombo, Félix da Costa, Ismail, Cardoso, \& Figueira, 2015). In support of this, Kleiner et al. (2004) stated that food, alcohol and drugs all influence a common pathway. Furthermore, they concluded that overeating and in turn obesity may be acting as a protective factor in limiting the consumption of alcohol and the use of drugs as food is sufficiently providing the rewards they need, or in this case, providing suitable emotional regulation. Therefore, cutting back on one may lead to increases in another. For example, individuals who eat in response to emotions but then limit this 
may increase their consumption of alcohol to maintain a similar pattern of emotion regulation. Future research should examine how the model presented in this thesis may be adapted to provide direct and indirect influences on other maladaptive behaviours.

To expand on this further, Finch and Tomiyama (2015) found that individuals who comfort ate attenuated their perceived stress following adverse life events. However, this 'buffering effect' was not found in individuals who also had elevated levels of depression. This suggests that in some situations, emotional eating may serve as an adaptive mechanism to cope with stressful events even though it is not a suitable strategy for individuals experiencing depression. This highlights that whilst it is important to reduce emotional eating, for some individuals emotional eating acts as a 'buffer' to stress and therefore could be seen as a beneficial mechanism for regulating emotions. For these individuals, it is not simply a case of reducing emotional eating but the development of a new strategy to help them cope with and reduce their emotions. Weight management is likely to differ if emotional eating is taken away as this is also removing the use of food to self-soothe. Therefore, addressing emotional dysregulation should be integral to interventions designed to manage weight.

This thesis explored how emotional dysregulation, via increased emotional eating, may have a negative impact on BMI. However, as previously mentioned, overeating is just one of many factors contributing to BMI and additional explanations for the associations between emotional dysregulation and BMI need to be explored. Evidence for this is provided by Study Two (Chapter Two) which found that alexithymia indirectly influenced BMI through negative affect, but this was not in serial with emotional eating. Therefore, this suggests that other behaviours may be impacting on BMI. For example, when the relationship is mediated by depression scores, it may be that individuals lack the motivation to exercise, or when the 
relationship is mediated by anxiety, individuals experience nerves about partaking in exercise, both of which may result in increased BMI. In addition, individuals may engage in other maladaptive coping mechanisms that can have a negative impact on BMI, such as alcohol consumption and smoking. One factor that may be particularly significant is alcohol consumption. Alcohol consumption is attributable to increased BMI and obesity (Lahti-Koski, Pietinen, Heliovaara, \& Vartiainen, 2002; Lukasiewicz et al., 2005; Suter \& Tremblay, 2005; Wannamethee \& Shaper, 2003) with alcohol consumption in response to emotions associated with higher BMI (Laitinen et al., 2002). Laitinen et al. (2002) found that BMI was highest in participants who consumed food and alcohol in response to stress, particularly in females. Furthermore, they found increased eating in response to stress was associated with increased drinking, further supporting the idea that food and alcohol consumption are behaviours used to regulate emotions. These results support the suggestion of exploring how the theoretical model developed in this thesis may be applicable to other behaviours (as stated at the start of this section) and suggest other behaviours, such as alcohol consumption should be considered when investigating the associations between emotional eating and BMI.

Finally, developing the concept that emotional regulation strategies need to be established (i.e. not turning to food or alcohol as a mechanism for managing emotions) as part of a healthy lifestyle, interventions are required to enable individuals to appropriately deal with their emotions. One possible avenue to consider is the development of coping strategies in children. Bruch (1973) stated it was essential for children to learn to correctly identify their bodily sensations and to be able to satisfy them in a socially and culturally acceptable manner. As previously mention in section 1.4., parental influences are associated with emotional eating in children, further supporting the need to enable children to appropriately manage their emotions and in 
turn, have a healthier lifestyle. Currently, preliminary studies have started to be conducted investigating interventions designed to improve the emotional connection between parents and children (“Tuning into kids;" Havighurst et al., 2013; Havighurst, Wilson, Harley, Prior, \& Kehoe, 2010) and increase a child's ability to cope with emotions and stress (Contextual Emotion-Regulation Therapy; Kovacs et al., 2006). So far, the "tuning into kids" intervention has led to significant improvements in a parents own emotional awareness and regulation and improved emotional knowledge (e.g. emotional identification, situational resolution) in children (Havighurst et al., 2013, 2010).

\subsubsection{Future directions}

This thesis explored the mediating factors of negative affect, negative urgency, interoceptive awareness and feeling fat in the relationship between emotional dysregulation and emotional eating. Consistently, it was found that emotional dysregulation was associated with emotional eating, but the emergence of a range of pathways between the two constructs mean the specific mechanisms that underpin emotional eating remain unclear. It is possible that additional factors may play a role. For example, there is emerging literature to suggest that anxiety sensitivity (Reaves et al., 2016) and distress tolerance (Anestis, Selby, Fink, \& Joiner, 2007; Eichen, Chen, Boutelle, \& McCloskey, 2017) may be associated with eating behaviours. Anxiety sensitivity, the fear of the physical symptoms of anxiety and the negative consequences of the associated symptoms (Reiss \& McNally, 1985), has been associated with alexithymia (e.g. Devine, Stewart, \& Watt, 1999; Wood, O’Hagan, Williams, McCabe, \& Chadwick, 2014), and it may be that individuals who are uncomfortable with the 
associated sensations of anxiety may be more inclined to try to remove them as quickly as possible through maladaptive coping strategies such as emotional eating.

In addition to anxiety sensitivity, a concept that is associated with more general discomfort following negative affect is distress tolerance. Distress tolerance is defined as the degree to which the experience of negative affect is deemed unbearable (Brown, Lejuez, Kahler, Strong, \& Zvolensky, 2005) or in other words, a general difficulty tolerating emotional distress (Anestis, Selby, Fink, \& Joiner, 2007). The concept is closely linked to negative urgency and acting rashly in response to negative affect, following the idea that if you have low distress tolerance you are more likely to act in a way to alleviate quicker (Anestis et al., 2007). Research exploring the relationship between distress tolerance and eating behaviours is beginning to attract interest, particularly in association with eating disorders. Anestis and colleagues (2007) found that distress tolerance significantly predicted EDI-Bulimia scores and a distress tolerance $\mathrm{x}$ negative urgency interaction also significantly predicted EDI-Bulimia. The authors concluded that high negative urgency and low distress tolerance scores were mostly likely to result in high EDI-Bulimia scores. In mediation analysis they also found that distress tolerance mediated the relationship between anxiety sensitivity and EDI-Bulimia. In addition, Eichen et al. (2017) found that individuals with BED exited a behavioural measure of distress tolerance (Paced Auditory Serial Addition Task) significantly quicker than healthy controls. Specifically, individuals with BED appeared to be less able to tolerate the stress caused by the task than healthy controls. Here, to alleviate the stress, participants had to simply 'quit' the task, but it may be that outside of the laboratory, individuals consume food as a way of alleviating stress and individuals who are less able to tolerate distress chose to more frequently engage in emotional eating. 
Insecure attachment style has also been significantly associated with an increased susceptibility to stress and increased use of external regulators of affect (Maunder \& Hunter, 2001). Therefore, this is a third factor that may be worth considering in the development of future models. Evidence for this is provided by a number of studies. Firstly, healthy participants consumed more cookies following an experimental prime that induced attachment anxiety compared to secure attachment (Wilkinson et al., 2013). Secondly, attachment anxiety was found to be a significant predictor of BMI and disinhibited eating fully mediated the relationship between attachment anxiety and BMI (Wilkinson, Rowe, Bishop, \& Brunstrom, 2010). Finally, emotional eating significantly mediated the association between attachment anxiety and BMI and poor emotion management (Wilkinson, Rowe, Robinson, \& Hardman, 2018). This suggests that individuals with an insecure attachment style may feel unable to effectively manage their emotions and in turn engage in emotional eating, potentially resulting in increased BMI. This may fit into the model through providing an underlying trait that increases an individual's vulnerability to reacting rashly in response to emotional eating or difficulty trusting their bodily sensations.

Currently, this thesis provides an initial theoretical model which partly uncovers the underlying mechanisms of emotional eating and in turn BMI. However, due to inconsistent findings across studies there is scope for additional factors to be explored. Based on available literature, anxiety sensitivity, distress tolerance and attachment style are suggested factors that warrant further investigation. These factors may help uncover the specific influences on emotional eating. 


\subsection{Closing statement}

Obesity remains a significant public health challenge. This thesis highlights the importance of understanding the role that emotion regulation plays in eating behaviour. Although the precise nature of the way in which emotional dysregulation drives overeating is far from clear, this thesis takes the first steps in developing one inclusive model. Alexithymia, used as a proxy for emotional dysregulation, was found to directly, and indirectly, influence emotional eating. The indirect pathways between alexithymia and emotional eating were mediated by negative affect (depression and anxiety), negative urgency, interoceptive awareness and feeling fat. The next steps will be to test whether the model developed is applicable to other maladaptive behaviours and further investigate factors which may also underpin the mechanisms of emotional eating. Furthermore, as emotion regulation is a pivotal factor in driving maladaptive behaviours, it is crucial that emotional dysregulation is considered in the development of interventions. Interventions designed to support weight loss/management and reduce alcohol consumption are unlikely to be successful it they neglect the role of emotion. 


\section{References}

Aaker, J., Drolet, A., \& Griffin, D. (2008). Recalling Mixed Emotions. Journal of Consumer Research, 35(2), 268.

Aarts, H., \& Dijksterhuis, A. (2000). Habits as knowledge structures: Automaticity in goal-directed behavior. Journal of Personality and Social Psychology, 78(1), 5363. doi:10.1037/0022-3514.78.1.53

Abarca-Gómez, L., Abdeen, Z. A., Hamid, Z. A., Abu-Rmeileh, N. M., AcostaCazares, B., Acuin, C., ... Ezzati, M. (2017). Worldwide trends in body-mass index, underweight, overweight, and obesity from 1975 to 2016: a pooled analysis of 2416 population-based measurement studies in 128.9 million children, adolescents, and adults. The Lancet, 390(10113), 2627-2642. doi:10.1016/S0140-6736(17)32129-3

Adriaanse, M. A., de Ridder, D. T. D., \& Evers, C. (2011). Emotional eating: Eating when emotional or emotional about eating? Psychology and Health, 26(1), 2339. doi:10.1080/08870440903207627

Afshin, A., Micha, R., Khatibzadeh, S., Fahimi, S., Shi, P., Powles, J., ... Mozaffarian, D. (2015). The impact of dietary habits and metabolic risk factors on cardiovascular and diabetes mortality in countries of the Middle East and North Africa in 2010: A comparative risk assessment analysis. BMJ Open, 5(5), e006385-e006385. doi:10.1136/bmjopen-2014-006385

Ainley, V., Maister, L., Brokfeld, J., Farmer, H., \& Tsakiris, M. (2013). More of myself: Manipulating interoceptive awareness by heightened attention to bodily and narrative aspects of the self. Consciousness and Cognition, 22(4), 12311238. doi:10.1016/j.concog.2013.08.004

Ainley, V., Tajadura-Jiménez, A., Fotopoulou, A., \& Tsakiris, M. (2012). Looking 
into myself: Changes in interoceptive sensitivity during mirror self-observation. Psychophysiology, 49(11), 1672-1676. doi:10.1111/j.1469-8986.2012.01468.x

Ainslie, G. (1975). Specious reward: A behavioral theory of impulsiveness and impulse control. Psychological Bulletin, 82(4), 463-496. doi:10.1037/h0076860

Ainslie, G. (2001). Breakdown of Will. Cambridge, UK: Cambridge University Press.

Alpaslan, A. H., Soylu, N., AVCI, K., Coşkun, K. Ş., Kocak, U., \& Taş, H. U. (2015). Disordered eating attitudes, alexithymia and suicide probability among Turkish high school girls. Psychiatry Research, 226(1), 224-229. doi:10.1016/J.PSYCHRES.2014.12.052

American Psychiatric Association. (2013). Diagnostic and statistical manual of mental disorders : DSM-5. Washington, USA: American Psychiatry Association.

Andersen, A. E. (2000). "How i Practice" Responding to the phrase "I feel fat". Eating Disorders, 8(2), 167-169. doi:10.1080/10640260008251223

Anestis, M. D., Selby, E. A., Fink, E. L., \& Joiner, T. E. (2007). The multifacted role of distress tolerance in dysregulated eating behaviours. The International Journal of Eating Disorders, 40(8), 718-726. doi:10.1002/eat.20471

Anestis, M. D., Selby, E. A., \& Joiner, T. E. (2007). The role of urgency in maladaptive behaviors. Behaviour Research and Therapy, 45(12), 3018-3029. doi:10.1016/j.brat.2007.08.012

Anestis, M. D., Smith, A. R., Fink, E. L., \& Joiner, T. E. (2009). Dysregulated eating and distress: Examining the specific role of negative urgency in a clinical sample. Cognitive Therapy and Research, 33(4), 390-397. doi:10.1007/s10608-008$9201-2$

Appelhans, B. M., Waring, M. E., Schneider, K. L., Sherry, L., Debiasse, M. A., \& Whited, M. C. (2013). Delay discounting and intake of ready-to-eat and away- 
from- home foods in overweight and obese women. Appetite, 59(2), 576-584. doi:10.1016/j.appet.2012.07.009.Delay

Appelhans, B. M., Whited, M. C., Schneider, K. L., Oleski, J., \& Pagoto, S. L. (2011). Response style and vulnerability to anger-induced eating in obese adults. Eating Behaviors, 12(1), 9-14. doi:10.1016/j.eatbeh.2010.08.009

Arnow, B., Kenardy, J., \& Agras, W. S. (1995). The emotional eating scale: The development of a measure to assess coping with negative affect by eating. International Journal of Eating Disorders, 18(1), 79-90. doi:10.1002/1098108X(199507)18:1<79::AID-EAT2260180109>3.0.CO;2-V

Avila, C. (2001). Distinguishing BIS-mediated and BAS-mediated disinhibition mechanisms: A comparison of disinhibition models of Gray $(1981,1987)$ and of Patterson and Newman (1993). Journal of Personality and Social Psychology, 80(2), 311-324. doi:10.1037/0022-3514.80.2.311

Bach, M., Bach, D., Böhmer, F., \& Nutzinger, D. O. (1994). Alexithymia and somatization: Relationship to DSM-III-R diagnoses. Journal of Psychosomatic Research, 38(6), 529-538. doi:10.1016/0022-3999(94)90050-7

Bagby, R. M., Parker, J. D. A., \& Taylor, G. J. (1994). The twenty-item Toronto Alexithymia Scale-I. Item selection and cross-validation of the factor structure. Journal of Psychosomatic Research, 38(1), 23-32. doi:10.1016/00223999(94)90005-1

Bagby, R. M., Taylor, G. J., \& Parker, J. D. A. (1994). The twenty-item Toronto Alexithymia Scale-II. Convergent, discriminant, and concurrent validity. Journal of Psychosomatic Research, 38(1), 33-40. doi:10.1016/00223999(94)90006-X

Bain, A. (1859). The Emotions and the Will. London, UK: Parker. doi:10.1037/10617- 
000

Bar-On, R. (2004). The Bar-On Emotional Quotient Inventory (EQ-i): Rationale, Description, and Summary of Psychometric Properties. In Measuring emotional intelligence: common ground and controversy (pp. 115-145).

Barkley, R. A. (1997). Behavioral inhibition, sustained attention, and executive functions: Constructing a unifying theory of ADHD. Psychological Bulletin, 121(1), 65-94. doi:10.1037/0033-2909.121.1.65

Barrett, L. F. (2006). Valence is a basic building block of emotional life. Journal of Research in Personality, 40(1), 35-55. doi:10.1016/J.JRP.2005.08.006

Barrett, L. F., \& Bliss-Moreau, E. (2009). Affect as a Psychological Primitive. In M. P. Zanna (Ed.), Advances in Experimental Social Psychology (Vol. 41, pp. 167218). Burlington, UK: Academic Press. doi:10.1016/S0065-2601(08)00404-8

Baumeister, R. F., Heatherton, T. F., \& Tice, D. M. (1994). Losing Control: How and Why People Fail at Self-Regulation. San Diego, USA: Academic Press.

Beales, D. L., \& Dolton, R. (2000). Eating disordered patients: personality, alexithymia, and implications for primary care. British Journal of General Practice, 50(450), 21-26.

Beck, A. T., Epstein, N., Brown, G., \& Steer, R. A. (1988). An inventory for measuring clinical anxiety: Psychometric properties. Journal of Consulting and Clinical Psychology, 56(6), 893-897. doi:10.1037/0022-006X.56.6.893

Beck, A. T., \& Steer, R. A. (1990). Manual for the Beck Anxiety Inventory. San Antonio, USA: Psychological Corporation.

Beck, A. T., Steer, R. A., \& Brown, G. K. (1996). Beck Depression Inventory-II. San Antonio, USA: Psychological Corporation.

Beck, A. T., Steer, R. A., \& Carbin, M. G. (1988). Psychometric properties of the Beck 
Depression Inventory: Twenty-five years of evaluation. Clinical Psychology Review, 8(1), 77-100. doi:10.1016/0272-7358(88)90050-5

Beck, A. T., Ward, C. H., Mendelson, M., Mock, J., \& Erbaugh, J. (1961). An Inventory for Measuring Depression. Archives of General Psychiatry, 4(6), 561571. doi:10.1001/archpsyc.1961.01710120031004

Begg, C. B., \& Mazumdar, M. (1994). Operating Characteristics of a Rank Correlation Test for Publication Bias. Biometrics, 50(4), 1088. doi:10.2307/2533446

Bekker, M. H. J., van de Meerendonk, C., \& Mollerus, J. (2004). Effects of negative mood induction and impulsivity on self-perceived emotional eating. International Journal of Eating Disorders, 36(4), 461-469. doi:10.1002/eat.20041

Ben-Tovim, D. I., \& Walker, M. K. (1991). The development of the Ben-Tovim Walker Body Attitudes Questionnaire (BAQ), a new measure of women's attitudes towards their own bodies. Psychological Medicine, 21(3), 775-784. doi:10.1017/S0033291700022406

Bennett, J., Greene, G., \& Schwartz-Barcott, D. (2013). Perceptions of emotional eating behavior. A qualitative study of college students. Appetite, 60, 187-192. doi:10.1016/J.APPET.2012.09.023

Bennett, P. (2001). Mood Disorders. In P. Bennett (Ed.), Abnormal and Clinical Psychology: An Introductory Textbook 3rd Edition. Berkshire, UK: Open University Press.

Bennett, P. (2015). Disorders of Mood. In P. Bennett (Ed.), Clinical Psychology: Psychopathology Through the Lifespan. Berkshire, UK: Open University Press. Berrios, R., Totterdell, P., \& Kellett, S. (2013). Validation of new a mixed emotions scale. In Annual Spring Conference of the White Rose Consortium.

Berrios, R., Totterdell, P., \& Kellett, S. (2015a). Eliciting mixed emotions: A meta- 
analysis comparing models, types and measures. Frontiers in Psychology, 6(MAR), 428. doi:10.3389/fpsyg.2015.00428

Berrios, R., Totterdell, P., \& Kellett, S. (2015b). Investigating goal conflict as a source of mixed emotions. Cognition and Emotion, 29(4), 755-763. doi:10.1080/02699931.2014.939948

Berrios, R., Totterdell, P., \& Kellett, S. (2017). Individual differences in mixed emotions moderate the negative consequences of goal conflict on life purpose. Personality and Individual Differences, 110, 18-22. doi:10.1016/j.paid.2017.01.013

Berrios, R., Totterdell, P., \& Kellett, S. (2018). When Feeling Mixed Can Be Meaningful: The Relation Between Mixed Emotions and Eudaimonic WellBeing. Journal of Happiness Studies, 19(3), 841-861. doi:10.1007/s10902-0179849-y

Berthoz, S., Consoli, S., Perez-Diaz, F., \& Jouvent, R. (1999). Alexithymia and anxiety: Compounded relationships? A psychometric study. European Psychiatry, 14(7), 372-378. doi:10.1016/S0924-9338(99)00233-3

Berthoz, S., Haviland, M. G., Riggs, M. L., Perdereau, F., \& Bungener, C. (2005). Assessing alexithymia in French-speaking samples: Psychometric properties of the Observer Alexithymia Scale-French translation. European Psychiatry, 20(7), 497-502. doi:10.1016/j.eurpsy.2004.10.001

Berthoz, S., Perdereau, F., Godart, N., Corcos, M., \& Haviland, M. G. (2007). Observer- and self-rated alexithymia in eating disorder patients: Levels and correspondence among three measures. Journal of Psychosomatic Research, 62(3), 341-347. doi:10.1016/J.JPSYCHORES.2006.10.008

Bickel, W. K., \& Marsch, L. A. (2001). Toward a behavioral economic understanding 
of drug dependence: delay discounting processes. Addiction, 96(1), 73-86. doi:10.1046/j.1360-0443.2001.961736.x

Blair, A. J., Lewis, V. J., \& Booth, D. A. (1990). Does emotional eating interfere with success in attempts at weight control? Appetite, 15(2), 151-157. doi:10.1016/0195-6663(90)90047-C

Bonato, P., \& Boland, F. J. (1983). Delay of gratification in obese children. Addictive Behaviors, 8, 71-74. doi:10.1016/0306-4603(83)90059-X

Bongers, P., de Graaff, A., \& Jansen, A. (2016). 'Emotional' does not even start to cover it: Generalization of overeating in emotional eaters. Appetite, 96, 611-616. doi:10.1016/j.appet.2015.11.004

Bongers, P., Jansen, A., Havermans, R., Roefs, A., \& Nederkoorn, C. (2013). Happy eating: The underestimated role of overeating in a positive mood. Appetite, 67, 74-80. doi:10.1016/j.appet.2013.03.017

Boon, B., Stroebe, W., Schut, H., \& Jansen, A. (1998). Food for thought: Cognitive regulation of food intake. British Journal of Health Psychology, 3(1), 27-40. doi:10.1111/j.2044-8287.1998.tb00553.x

Braden, A., Flatt, S. W., Boutelle, K. N., Strong, D., Sherwood, N. E., \& Rock, C. L. (2016). Emotional eating is associated with weight loss success among adults enrolled in a weight loss program. Journal of Behavioral Medicine, 39(4), 727732. doi:10.1007/s10865-016-9728-8

Braden, A., Musher-Eizenman, D., Watford, T., \& Emley, E. (2018). Eating when depressed, anxious, bored, or happy: Are emotional eating types associated with unique psychological and physical health correlates? Appetite, 125, 410-417. doi:10.1016/j.appet.2018.02.022

Braet, C., \& Van Strien, T. (1997). Assessment of emotional, externally induced and 
restrained eating behaviour in nine to twelve-year-old obese and non-obese children. Behaviour Research and Therapy, 35(9), 863-873. doi:10.1016/S00057967(97)00045-4

Brondel, L., Romer, M., Van Wymelbeke, V., Pineau, N., Jiang, T., Hanus, C., \& Rigaud, D. (2009). Variety enhances food intake in humans: Role of sensoryspecific satiety. doi:10.1016/j.physbeh.2009.01.019

Brown, R. A., Lejuez, C. W., Kahler, C. W., Strong, D. R., \& Zvolensky, M. J. (2005, September 1). Distress tolerance and early smoking lapse. Clinical Psychology Review. Pergamon. doi:10.1016/j.cpr.2005.05.003

Bruce, M. L., Seeman, T. E., Merrill, S. S., \& Blazer, D. G. (1994). The impact of depressive symptomatology on physical disability: MacArthur studies of successful aging. American Journal of Public Health, 84(11), 1796-1799. doi:10.2105/AJPH.84.11.1796

Bruch, H. (1964). Psychological Aspects of Overeating And Obesity. Psychosomatics, 5(5), 269-274. doi:10.1016/S0033-3182(64)72385-7

Bruch, H. (1973). Eating disorders. Obesity, anorexia nervosa, and the person within. New York, USA: Basic Books.

Bruch, H. (1978). The golden cage. The enigma of anorexia nervosa. Cambridge, USA: Harvard University Press. doi:dx.doi.org/10.1097/00017285-19780900000006

Bydlowski, S., Corcos, M., Jeammet, P., Paterniti, S., Berthoz, S., Laurier, C., ... Consoli, S. M. (2005). Emotion-processing deficits in eating disorders. International Journal of Eating Disorders, 37(4), 321-329.

Cabello, R., \& Fernández-Berrocal, P. (2015). Implicit theories and ability emotional intelligence. Frontiers in Psychology, 6, 700. doi:10.3389/fpsyg.2015.00700 
Calder, A. J., Keane, J., Manly, T., Sprengelmeyer, R., Scott, S., Nimmo-Smith, I., \& Young, A. W. (2003). Facial expression recognition across the adult life span. Neuropsychologia, 41(2), 195-202. doi:10.1016/S0028-3932(02)00149-5

Cameron, O. G. (2002). Visceral Sensory Neuroscience: Interoception. Oxford, UK: Oxford University Press.

Carano, A., De Berardis, D., Campanella, D., Serroni, N., Ferri, F., Di Iorio, G., ... Di Giannantonio, M. (2012). Alexithymia and Suicide Ideation in a Sample of Patients with Binge Eating Disorder. Journal of Psychiatric Practice, 18(1), 511. doi:10.1097/01.pra.0000410982.08229.99

Carano, A., De Berardis, D., Gambi, F., Di Paolo, C., Campanella, D., Pelusi, L., ... Ferro, F. M. (2006). Alexithymia and body image in adult outpatients with binge eating disorder. International Journal of Eating Disorders, 39(4), 332-340.

Cardi, V., Esposito, M., Clarke, A., Schifano, S., \& Treasure, J. (2015). The impact of induced positive mood on symptomatic behaviour in eating disorders. An experimental, $\mathrm{AB} / \mathrm{BA}$ crossover design testing a multimodal presentation. Appetite, 87, 192-198. doi:10.1016/j.appet.2014.12.224

Cardi, V., Leppanen, J., \& Treasure, J. (2015). The effects of negative and positive mood induction on eating behaviour: A meta-analysis of laboratory studies in the healthy population and eating and weight. Neuroscience \& Biobehavioral Reviews. doi:10.1016/j.neubiorev.2015.08.011

Cartwright, M., Wardle, J., Steggles, N., Simon, A. E., Croker, H., \& Jarvis, M. J. (2003). Stress and dietary practices in adolescents. Health Psychology, 22(4), 362-369. doi:10.1037/0278-6133.22.4.362

Carver, C. S., \& Scheier, M. F. (1990). Origins and Functions of Positive and Negative Affect: A Control-Process View. Psychological Review, 97(1), 19-35. 
doi:10.1037/0033-295X.97.1.19

Cerny, C. (2017). The Costs of Obesity. Retrieved from http://obesityhealthalliance.org.uk/wp-content/uploads/2017/10/OHA-briefingpaper-Costs-of-Obesity-.pdf

Childers, M., \& Herzog, H. (2009). Motivations for Meat Consumption Among ExVegetarians. In Meeting of the International Society of Anthrozoology.

Christensen, L. (2001). The effect of food intake on mood. Clinical Nutrition, 20, 161166. doi:10.1054/clnu.2001.0420

Chua, J. L., Touyz, S., \& Hill, A. J. (2004). Negative mood-induced overeating in obese binge eaters: an experimental study. International Journal of Obesity, 28(4), 606-610. doi:10.1038/sj.ijo.0802595

Claes, L., Vandereycken, W., \& Vertommen, H. (2002). Impulsive and compulsive traits in eating disordered patients compared with controls. Personality and Individual Differences, 32(4), 707-714. doi:10.1016/S0191-8869(01)00071-X

Claes, L., Vandereycken, W., \& Vertommen, H. (2005). Impulsivity-related traits in eating disorder patients. Personality and Individual Differences, 39(4), 739-749. doi:10.1016/J.PAID.2005.02.022

Clyne, C., \& Blampied, N. M. (2004). Training in emotion regulation as a treatment for binge eating: A preliminary study. Behaviour Change, 21(4), 269-281. doi:10.1375/bech.21.4.269.66105

Cochrane, C. E., Brewerton, T. D., Wilson, D. B., \& Hodges, E. L. (1993). Alexithymia in the eating disorders. International Journal of Eating Disorders, 14(2), 219-222. doi:10.1002/1098-108X(199309)14:2<219::AIDEAT2260140212>3.0.CO;2-G

Cole, P. M., Michel, M. K., \& Teti, L. O. (1994). The development of emotion 
regulation and dysregulation: A clinical perspective. Monographs of the Society for Research in Child Development, 59(2-3), 73-102. doi:10.1111/j.15405834.1994.tb01278.x

Cooper, M. J., Deepak, K., Grocutt, E., \& Bailey, E. (2007). The experience of 'feeling fat' in women with anorexia nervosa, dieting and non-dieting women: an exploratory study. European Eating Disorders Review, 15(5), 366-372. doi:10.1002/erv.785

Cooper, M. L., Russell, M., Skinner, J. B., Frone, M. R., \& Mudar, P. (1992). Stress and Alcohol Use: Moderating Effects of Gender, Coping, and Alcohol Expectancies. Journal of Abnormal Psychology, 101(1), 139-152. doi:10.1037/0021-843X.101.1.139

Corcos, M., Guilbaud, O., Speranza, M., Paterniti, S., Loas, G., Stephan, P., \& Jeammet, P. (2000). Alexithymia and depression in eating disorders. Psychiatry Research, 93(3), 263-266. doi:10.1016/S0165-1781(00)00109-8

Corsica, J., Hood, M. M., Katterman, S., Kleinman, B., \& Ivan, I. (2014). Development of a novel mindfulness and cognitive behavioral intervention for stress-eating: A comparative pilot study. Eating Behaviors, 15(4), 694-699. doi:10.1016/J.EATBEH.2014.08.002

Costa, P. T., \& McCrae, R. R. (1992). NEO Personality Inventory-Revised (NEO PIR). Odessa, USA: Psychological Assessment Resources.

Craig, A. D. (2002). How do you feel? Interoception: the sense of the physiological condition of the body. Nature Reviews Neuroscience, 3(8), 655-666. doi:10.1038/nrn894

Craig, A. D. D. (2005, December 1). Forebrain emotional asymmetry: A neuroanatomical basis? Trends in Cognitive Sciences. Elsevier Current Trends. 
doi:10.1016/j.tics.2005.10.005

Crawford, J. R., \& Henry, J. D. (2004). The Positive and Negative Affect Schedule (PANAS): Construct validity, measurement properties and normative data in a large non-clinical sample. British Journal of Clinical Psychology, 43(3), 245265. doi:10.1348/0144665031752934

Critchfield, T. S., \& Kollins, S. H. (2001). Temporal discounting: basic research and the analysis of socially important behavior. Journal of Applied Behavior Analysis, 34(1), 101-122. doi:10.1901/jaba.2001.34-101

Critchley, H. D., Wiens, S., Rotshtein, P., Öhman, A., \& Dolan, R. J. (2004). Neural systems supporting interoceptive awareness. Nature Neuroscience, 7(2), 189195. doi:10.1038/nn1176

Crockett, A. C., Myhre, S. K., \& Rokke, P. D. (2015). Boredom proneness and emotion regulation predict emotional eating. Journal of Health Psychology, 20(5), 670 680. doi:10.1177/1359105315573439

Crosby, R. D., Wonderlich, S. A., Engel, S. G., Simonich, H., Smyth, J., \& Mitchell, J. E. (2009). Daily mood patterns and bulimic behaviors in the natural environment. Behaviour Research and Therapy, 47(3), 181-188. doi:10.1016/J.BRAT.2008.11.006

Cross, C. P., Copping, L. T., \& Campbell, A. (2011). Sex differences in impulsivity: A meta-analysis. Psychological Bulletin, 137(1), 97-130. doi:10.1037/a0021591

Crowther, J. H., Sanftner, J., Bonifazi, D. Z., \& Shepherd, K. L. (2001). The role of daily hassles in binge eating. International Journal of Eating Disorders, 29(4), 449-454. doi:10.1002/eat.1041

Cyders, M. A., \& Coskunpinar, A. (2010). Is urgency emotionality? Separating urgent behaviors from effects of emotional experiences. Personality and Individual 
Differences, 48(7), 839-844. doi:10.1016/J.PAID.2010.02.009

Cyders, M. A., \& Coskunpinar, A. (2011a). Depression, impulsivity and health-related disability: A moderated mediation analysis. Journal of Research in Personality, 45(6), 679-682. doi:10.1016/J.JRP.2011.08.005

Cyders, M. A., \& Coskunpinar, A. (2011b). Measurement of constructs using selfreport and behavioral lab tasks: Is there overlap in nomothetic span and construct representation for impulsivity? Clinical Psychology Review. doi:10.1016/j.cpr.2011.06.001

Cyders, M. A., \& Smith, G. T. (2007). Mood-based rash action and its components: Positive and negative urgency. Personality and Individual Differences, 43(4), 839-850. doi:10.1016/j.paid.2007.02.008

Cyders, M. A., \& Smith, G. T. (2010). Longitudinal validation of the urgency traits over the first year of college. Journal of Personality Assessment, 92(1), 63-69. doi:10.1080/00223890903381825

Cyders, M. A., Smith, G. T., Spillane, N. S., Fischer, S., Annus, A. M., \& Peterson, C. (2007). Integration of impulsivity and positive mood to predict risky behavior: development and validation of a measure of positive urgency. Psychological Assessment, 19(1), 107-118. doi:10.1037/1040-3590.19.1.107

D’Argembeau, A., Comblain, C., \& van der Linden, M. (2003). Phenomenal characteristics of autobiographical memories for positive, negative and neutral events. Applied Cognitive Psychology, 17, 281-294. doi:10.1002/acp.856

D’Zurilla, T. J., Nezu, A. M., \& Maydeu-Olivares, A. (2002). Manual for the Social Problem-Solving Inventory-Revised. North Tonawanda, USA: Multi-Health Systems.

Damasio, A. R. (1994). Descartes' error: Emotion, rationality and the human brain. 
New York, USA: Putnam (Grossets Books).

Daniel, T. O., Stanton, C. M., \& Epstein, L. H. (2013). The future is now: Comparing the effect of episodic future thinking on impulsivity in lean and obese individuals. Appetite, 71, 120-125. doi:10.1016/j.appet.2013.07.010

Davis-Becker, K., Peterson, C. M., \& Fischer, S. (2014). The relationship of trait negative urgency and negative affect to disordered eating in men and women. Personality and Individual Differences, 56, 9-14. doi:10.1016/J.PAID.2013.08.010

Davis, C., Levitan, R. D., Smith, M., Tweed, S., \& Curtis, C. (2006). Associations among overeating, overweight, and attention deficit/hyperactivity disorder: A structural equation modelling approach. Eating Behaviors, 7(3), 266-274. doi:10.1016/j.eatbeh.2005.09.006

Davis, C., Loxton, N. J., Levitan, R. D., Kaplan, A. S., Carter, J. C., \& Kennedy, J. L. (2013). 'Food addiction' and its association with a dopaminergic multilocus genetic profile. Physiology \& Behavior, 118, 63-69. doi:10.1016/J.PHYSBEH.2013.05.014

Davis, C., Patte, K., Curtis, C., \& Reid, C. (2010). Immediate pleasure and future consequences. A neuropsychological study of binge eating and obesity. Appetite, 54, 208-213. doi:10.1016/j.appet.2009.11.002

Davis, C., Patte, K., Levitan, R., Reid, C., Tweed, S., \& Curtis, C. (2007). From motivation to behaviour: A model of reward sensitivity, overeating, and food preferences in the risk profile for obesity. Appetite, 48(1), 12-19. doi:10.1016/j.appet.2006.05.016

Davis, K. R., \& Fischer, S. (2013). The influence of trait anger, trait anxiety and negative urgency on disordered eating. Personality and Individual Differences, 
54(2), 307-310. doi:10.1016/j.paid.2012.08.036

Davis, R., Freeman, R. J., \& Garner, D. M. (1988). A Naturalistic Investigation of Eating Behavior in Bulimia Nervosa. Journal of Consulting and Clinical Psychology, 56(2), 273-279. doi:10.1037/0022-006X.56.2.273

Dawe, S., \& Loxton, N. J. (2004). The role of impulsivity in the development of substance use and eating disorders. Neuroscience \& Biobehavioral Reviews, 28(3), 343-351. doi:10.1016/J.NEUBIOREV.2004.03.007

De Berardis, D., Carano, A., Gambi, F., Campanella, D., Giannetti, P., Ceci, A., ... Ferro, F. M. (2007). Alexithymia and its relationships with body checking and body image in a non-clinical female sample. Eating Behaviors, 8(3), 296-304. doi:10.1016/j.eatbeh.2006.11.005

de Boer, M. R., Waterlander, W. E., Kuijper, L. D., Steenhuis, I. H., \& Twisk, J. W. (2015). Testing for baseline differences in randomized controlled trials: an unhealthy research behavior that is hard to eradicate. International Journal of Behavioral Nutrition and Physical Activity, 12(1), 4. doi:10.1186/s12966-015$0162-\mathrm{z}$

de Lauzon, B., Romon, M., Deschamps, V., Lafay, L., Borys, J.-M., Karlsson, J., ... Charles, M. A. (2004). The Three-Factor Eating Questionnaire-R18 is able to distinguish among Ddfferent eating patterns in a general population. The Journal of Nutrition, 134(9), 2372-2380. doi:10.1093/jn/134.9.2372

De Silva, P., \& Eysenck, S. (1987). Personality and addictiveness in anorexic and bulimic patients. Personality and Individual Differences, 8(5), 749-751. doi:10.1016/0191-8869(87)90077-8

Deckman, T., \& DeWall, C. (2011). Negative urgency and risky sexual behaviors: A clarification of the relationship between impulsivity and risky sexual behavior. 
Personality and Individual Differences. Retrieved from http://www.sciencedirect.com/science/article/pii/S0191886911002807

Delgado-Rico, E., Río-Valle, J. S., González-Jiménez, E., Campoy, C., \& VerdejoGarcía, A. (2012). BMI Predicts Emotion-Driven Impulsivity and Cognitive Inflexibility in Adolescents With Excess Weight. Obesity, 20(8), 1604-1610. doi:10.1038/oby.2012.47

Devine, H., Stewart, S. H., \& Watt, M. C. (1999). Relations between anxiety sensitivity and dimensions of alexithymia in a young adult sample. Journal of Psychosomatic Research, 47(2), 145-158. doi:10.1016/S0022-3999(99)00033-1

Di Cesare, M., Bentham, J., Stevens, G. A., Zhou, B., Danaei, G., Lu, Y., ... Cisneros, J. Z. (2016, April 2). Trends in adult body-mass index in 200 countries from 1975 to 2014: A pooled analysis of 1698 population-based measurement studies with 19.2 million participants. The Lancet. Elsevier. doi:10.1016/S01406736(16)30054-X

Diehl, M., Coyle, N., \& Labouvie-Vief, G. (1996). Age and Sex Differences in Strategies of Coping and Defense Across the Life Span. Psychology and Aging, 11(1), 127-139. doi:10.1037//0882-7974.11.1.127

Dingemans, A. E., Martijn, C., Jansen, A. T. M., \& van Furth, E. F. (2009). The effect of suppressing negative emotions on eating behavior in binge eating disorder. Appetite, 52(1), 51-57. doi:10.1016/j.appet.2008.08.004

Dingemans, A. E., Martijn, C., van Furth, E. F., \& Jansen, A. T. M. (2009). Expectations, mood, and eating behavior in binge eating disorder. Beware of the bright side. Appetite, 53(2), 166-173. doi:10.1016/j.appet.2009.06.002

Dir, A. L., Karyadi, K., \& Cyders, M. A. (2013). The uniqueness of negative urgency as a common risk factor for self-harm behaviors, alcohol consumption, and eating 
problems. $\quad$ Addictive Behaviors, 38(5), 2158-2162. doi:10.1016/j.addbeh.2013.01.025

Domoff, S. E., Meers, M. R., Koball, A. M., \& Musher-Eizenman, D. R. (2014). The validity of the Dutch Eating Behavior Questionnaire: Some critical remarks. Eating and Weight Disorders. doi:10.1007/s40519-013-0087-y

Douketis, J. D., \& Sharma, A. M. (2005). Obesity and Cardiovascular Disease: Pathogenic Mechanisms and Potential Benefits of Weight Reduction. Seminars in Vascular Medicine, 5(01), 25-33. doi:10.1055/s-2005-871739

Doya, K. (2008). Modulators of decision making. Nature Neuroscience, 11(4), 410 416. doi:10.1038/nn2077

Dubé, L., LeBel, J. L., \& Lu, J. (2005). Affect asymmetry and comfort food consumption. Physiology \& Behavior, 86(4), 559-567. doi:10.1016/J.PHYSBEH.2005.08.023

Ebneter, D., Latner, J., Rosewall, J., \& Chisholm, A. (2012). Impulsivity in restrained eaters: Emotional and external eating are associated with attentional and motor impulsivity. Eating and Weight Disorders-Studies on Anorexia, Bulimia and Obesity, 17(1), 62-65.

Egger, M., Smith, G. D., \& Phillips, A. N. (1997). Meta-analysis: principles and procedures. BMJ (Clinical Research Ed.), 315(7121), 1533-7. doi:10.1136/BMJ.315.7121.1533

Eichen, D. M., Chen, E., Boutelle, K. N., \& McCloskey, M. S. (2017). Behavioral evidence of emotion dysregulation in binge eaters. Appetite, 111, 1-6. doi:10.1016/J.APPET.2016.12.021

Eizaguirre, A. E., de Cabezón, A. O. S., de Alda, I. O., Olariaga, L. J., \& Juaniz, M. (2004). Alexithymia and its relationships with anxiety and depression in eating 
disorders. Personality and Individual Differences, 36(2), 321-331. doi:10.1016/S0191-8869(03)00099-0

Elmore, D. K., \& de Castro, J. M. (1990). Self-rated moods and hunger in relation to spontaneous eating behavior in bulimics, recovered bulimics, and normals. International Journal of Eating Disorders, 9(2), 179-190. doi:10.1002/1098108X(199003)9:2<179::AID-EAT2260090207>3.0.CO;2-O

Emery, R., King, K., \& Levine, M. (2014). The moderating role of negative urgency on the associations between affect, dietary restraint, and calorie intake: An experimental study. Personality and Individual Differences. Retrieved from http://www.sciencedirect.com/science/article/pii/S0191886913013421

Emery, R. L., King, K. M., Fischer, S. F., \& Davis, K. R. (2013). The moderating role of negative urgency on the prospective association between dietary restraint and binge eating. Appetite, 71, 113-119. doi:10.1016/j.appet.2013.08.001

Endler, N. S., \& Parker, J. D. A. (1994). Assessment of Multidimensional Coping: Task, Emotion, and Avoidance Strategies. Psychological Assessment, 6(1), 5060. doi:10.1037/1040-3590.6.1.50

Evers, C., Adriaanse, M., de Ridder, D. T. D., \& de Witt Huberts, J. C. (2013). Good mood food. Positive emotion as a neglected trigger for food intake. Appetite, 68, 1-7. doi:10.1016/j.appet.2013.04.007

Evers, C., de Ridder, D. T. D., \& Adriaanse, M. A. (2009). Assessing yourself as an emotional eater: mission impossible? Appetite, 28(6), 717-725. doi:10.1037/a0016700

Evers, C., Stok, F., \& Ridder, D. de. (2010). Feeding your feelings: Emotion regulation strategies and emotional eating. Personality and Social Psychology Bulletin, $X X(\mathrm{X}), 1-13$. 
Eysenck, S. B. G., Easting, G., \& Pearson, P. R. (1984). Age norms for impulsiveness, venturesomeness and empathy in children. Personality and Individual Differences, 5(3), 315-321. doi:10.1016/0191-8869(84)90070-9

Eysenck, S. B. G., Pearson, P. R., Easting, G., \& Allsopp, J. F. (1985). Age norms for impulsiveness, venturesomeness and empathy in adults. Personality and Individual Differences, 6(5), 613-619. doi:10.1016/0191-8869(85)90011-X

Fairburn, C. G. (2001). Eating Disorders. Encyclopedia of Life Sciences. Chichester, UK: John Wiley \& Sons, Ltd. doi:10.1038/npg.els.0002302

Fairburn, C. G., \& Beglin, S. J. (2008). Cognitive Behavior Therapy and Eating Disorders. New York, USA: Guildford Press.

Fairburn, C. G., \& Cooper, P. J. (1982). Self-induced vomiting and bulimia nervosa: an undetected problem. British Medical Journal (Clinical Research Ed.), 284(6323), 1153-5. doi:10.1136/BMJ.284.6323.1153

Fairclough, S. H., \& Goodwin, L. (2007). The effect of psychological stress and relaxation on interoceptive accuracy: Implications for symptom perception. Journal of Psychosomatic Research, 62(3), 289-295. doi:10.1016/j.jpsychores.2006.10.017

Faul, F., Erdfelder, E., Lang, A. G., \& Buchner, A. (2007). G*Power 3: A flexible statistical power analysis program for the social, behavioral, and biomedical sciences. Behavior Research Methods, 39, 175-191.

Fay, S., \& Finlayson, G. (2011). Negative affect-induced food intake in non-dieting women is reward driven and associated with restrained-disinhibited eating subtype. Appetite, 56(3), 682-686. doi:10.1016/j.appet.2011.02.004

Feingold, A. (1994). Gender differences in personality: A meta-analysis. Psychological Bulletin, 116(3), 429. doi:10.1037/0033-2909.116.3.429 
Fenton-O’Creevy, M., Dibb, S., \& Furnham, A. (2018). Antecedents and consequences of chronic impulsive buying: Can impulsive buying be understood as dysfunctional self-regulation? Psychology and Marketing, 35(3), 175-188. doi:10.1002/mar.21078

Ferriter, C., \& Ray, L. (2011). Binge eating and binge drinking: An integrative review. Eating Behaviors, 12, 99-107. doi:10.1016/j.eatbeh.2011.01.001

Finch, L. E., \& Tomiyama, A. J. (2015). Comfort eating, psychological stress, and depressive symptoms in young adult women. Appetite, 95, 239-244.

Fink, E. L., Anestis, M. D., Selby, E. A., \& Joiner, T. E. (2010). Negative urgency fully mediates the relationship between alexithymia and dysregulated behaviours. Personality and Mental Health, 4(4), 284-293. doi:10.1002/pmh.138

Fischer, H., Sandblom, J., Gavazzeni, J., Fransson, P., Wright, C. I., \& Bäckman, L. (2005). Age-differential patterns of brain activation during perception of angry faces. $\quad$ Neuroscience $\quad$ Letters, $\quad 386(2), \quad 99-104$. doi:10.1016/J.NEULET.2005.06.002

Fischer, S., Smith, G. T., \& Cyders, M. A. (2008). Another look at impulsivity: A meta-analytic review comparing specific dispositions to rash action in their relationship to bulimic symptoms. Clinical Psychology Review, 28(8), 14131425. doi:10.1016/J.CPR.2008.09.001

Fissler, M., Winnebeck, E., Schroeter, T., Gummersbach, M., Huntenburg, J. M., Gaertner, M., \& Barnhofer, T. (2016). An Investigation of the Effects of Brief Mindfulness Training on Self-Reported Interoceptive Awareness, the Ability to Decenter, and Their Role in the Reduction of Depressive Symptoms. Mindfulness, 7(5), 1170-1181. doi:10.1007/s12671-016-0559-z

Flegal, K. M., Carroll, M. D., Ogden, C. L., \& Curtin, L. R. (2010). Prevalence and 
Trends in Obesity Among US Adults, 1999-2008. JAMA, 303(3), 235. doi:10.1001/jama.2009.2014

Flegal, K. M., Carroll, M. D., Ogden, C. L., \& Johnson, C. L. (2002). Prevalence and trends in obesity among US adults, 1999-2000. Journal of the American Medical Association, 288(14), 1723-1727. doi:10.1001/jama.288.14.1723

Ford, J. H., Addis, D. R., \& Giovanello, K. S. (2012). Differential effects of arousal in positive and negative autobiographical memories. Memory, 20(7), 771-778. doi:10.1080/09658211.2012.704049

Fox, S., Conneely, S., \& Egan, J. (2017). Emotional expression and eating in overweight and obesity. Health Psychology and Behavioral Medicine, 5(1), 337357. doi:10.1080/21642850.2017.1378580

Frayn, M., \& Knäuper, B. (2017). Emotional Eating and Weight in Adults: a Review. Current Psychology, 1-10. doi:10.1007/s12144-017-9577-9

Freeman, L. M. Y., \& Gil, K. M. (2004). Daily stress, coping, and dietary restraint in binge eating. International Journal of Eating Disorders, 36(2), 204-212. doi:10.1002/eat.20012

Freyberger, H. (1977). Supportive psychotherapeutic techniques in primary and secondary alexithymia. Psychotherapy and Psychosomatics, 28(1-4), 337-42. doi: $10.1159 / 000287080$

Gabert-Quillen, C. A., Bartolini, E. E., Abravanel, B. T., \& Sanislow, C. A. (2015). Ratings for emotion film clips. Behavior Research Methods, 47(3), 773-787. doi:10.3758/s13428-014-0500-0

Gaher, R., Arens, A., \& Shishido, H. (2015). Alexithymia as a mediator between childhood maltreatment and impulsivity. Stress and Health. Retrieved from http://onlinelibrary.wiley.com/doi/10.1002/smi.2552/full 
Gaigg, S. B., Cornell, A. S., \& Bird, G. (2018). The psychophysiological mechanisms of alexithymia in autism spectrum disorder. Autism, 22(2), 227-231. doi:10.1177/1362361316667062

Galanti, K., Gluck, M. E., \& Geliebter, A. (2007). Test meal intake in obese binge eaters in relation to impulsivity and compulsivity. International Journal of Eating Disorders, 40(8), 727-732. doi:10.1002/eat.20441

Ganley, R. M. (1989). Emotion and eating in obesity: A review of the literature. International Journal of Eating Disorders, 8(3), 343-361. doi:10.1002/1098108X(198905)8:3<343::AID-EAT2260080310>3.0.CO;2-C

Garg, N., \& Lerner, J. S. (2013). Sadness and consumption. Journal of Consumer Psychology, 23(1), 106-113. doi:10.1016/j.jcps.2012.05.009

Garner, D. M. (1991). Eating Disorder Inventory-2. Odessa, USA: Psychological Assessment Resources.

Geliebter, A., \& Aversa, A. (2003). Emotional eating in overweight, normal weight, and underweight individuals. Eating Behaviors, 3(4), 341-347. doi:10.1016/S1471-0153(02)00100-9

Gibson, E. L. (2006). Emotional influences on food choice: Sensory, physiological and psychological pathways. Physiology \& Behavior, 89(1), 53-61. doi:10.1016/j.physbeh.2006.01.024

Gilboa-Schechtman, E., Avnon, L., Zubery, E., \& Jeczmien, P. (2006). Emotional processing in eating disorders: specific impairment or general distress related deficiency? Depression and Anxiety, 23(6), 331-339. doi:10.1002/da.20163

Girdhar, A., Mital, A., Kephart, A., \& Young, A. (2001). The Ecological Validity of Delay Aversion and Response Inhibition as Measures of Impulsivity in AD/HD: A Supplement to the NIMH Multimodal Treatment Study of AD/HD. Journal of 
Abnormal Child Psychology, 29(3), 215-228. Retrieved from https://link.springer.com/content/pdf/10.1023\%2FA\%3A1010329714819.pdf

Goldbacher, E., La Grotte, C., Komaroff, E., Vander Veur, S., \& Foster, G. D. (2016). An initial evaluation of a weight loss intervention for individuals who engage in emotional eating. Journal of Behavioral Medicine, 39(1), 139-150. doi:10.1007/s10865-015-9678-6

Goldschmidt, A. B., Lavender, J. M., Hipwell, A. E., Stepp, S. D., \& Keenan, K. (2017). Emotion Regulation and Loss of Control Eating in Community-Based Adolescents. Journal of Abnormal Child Psychology, 45(1), 183-191. doi:10.1007/s10802-016-0152-x

Goossens, L., Braet, C., Van Vlierberghe, L., \& Mels, S. (2009). Loss of control over eating in overweight youngsters: The role of anxiety, depression and emotional eating. European Eating Disorders Review, 17(1), 68-78. doi:10.1002/erv.892

Gratz, K. L., \& Roemer, L. (2004). Multidimensional Assessment of Emotion Regulation and Dysregulation: Development, Factor Structure, and Initial Validation of the Difficulties in Emotion Regulation Scale. In Journal of Psychopathology and Behavioral Assessment (Vol. 26, pp. 41-54). doi:10.1023/B:JOBA.0000007455.08539.94

Green, L., Fry, A. F., \& Myerson, J. (1994). Discounting of Delayed Rewards: A LifeSpan Comparison. Psychological Science, 5(1), 33-36. doi:10.1111/j.14679280.1994.tb00610.x

Green, L., Myerson, J., Lichtman, D., Rosen, S., \& Fry, A. (1996). Temporal discounting in choice between delayed rewards: The role of age and income. Psychology and Aging, 11(1), 79-84. doi:10.1037//0882-7974.11.1.79

Green, L., Myerson, J., \& Ostaszewski, P. (1999). Discounting of delayed rewards 
across the life span: age differences in individual discounting functions. Behavioural Processes, 46, 89-96.

Greene, G. W., Schembre, S. M., White, A. A., Hoerr, S. L., Lohse, B., Shoff, S., ... Blissmer, B. (2011). Identifying Clusters of College Students at Elevated Health Risk Based on Eating and Exercise Behaviors and Psychosocial Determinants of Body Weight. Journal of the American Dietetic Association, 111(3), 394-400. doi:10.1016/J.JADA.2010.11.011

Greeno, C. G., \& Wing, R. R. (1994). Stress-Induced Eating. Psychological Bulletin, 115(3), 444-464. doi:10.1037/0033-2909.115.3.444

Gross, J. J., \& Barrett, L. F. (2011). Emotion generation and emotion regulation: One or two depends on your point of view. Emotion Review, 3(1), 8-16. doi:10.1177/1754073910380974

Gross, J. J., \& Levenson, R. W. (1995). Emotion elicitation using films. Cognition \& Emotion, 9(1), 87-108. doi:10.1080/02699939508408966

Grucza, R. A., Przybeck, T. R., \& Cloninger, C. R. (2007). Prevalence and correlates of binge eating disorder in a community sample. Comprehensive Psychiatry, 48(2), 124-131. doi:10.1016/j.comppsych.2006.08.002

Guerrieri, R., Nederkoorn, C., \& Jansen, A. (2007). How impulsiveness and variety influence food intake in a sample of healthy women. Appetite, 48(1), 119-122. doi:10.1016/J.APPET.2006.06.004

Guerrieri, R., Nederkoorn, C., Stankiewicz, K., Alberts, H., Geschwind, N., Martijn, C., \& Jansen, A. (2007). The influence of trait and induced state impulsivity on food intake in normal-weight healthy women. Appetite, 49(1), 66-73. doi:10.1016/J.APPET.2006.11.008

Gunderson, J. G., \& Zanarini, M. C. (1989). Pathogenesis of borderline personality. 
Review of Psychiatry, 8, 25-48.

Gunning-Dixon, F. M., Gur, R. C., Perkins, A. C., Schroeder, L., Turner, T., Turetsky, B. I., ... Gur, R. E. (2003). Age-related differences in brain activation during emotional face processing. Neurobiology of Aging, 24(2), 285-295. doi:10.1016/S0197-4580(02)00099-4

Haedt-Matt, A., \& Keel, P. K. (2011). Revisiting the Affect Regulation Model of Binge Eating: A Meta-Analysis of Studies using Ecological Momentary Assessment. Psychological Bulletin, 137(4), 660-681. doi:10.1037/a0023660

Haghighi, M., Jahangard, L., Ahmadpanah, M., Bajoghli, H., Holsboer-Trachsler, E., \& Brand, S. (2016). The relation between anxiety and BMI - is it all in our $\begin{array}{llll}\text { curves? } & \text { Psychiatry } & \text { Research, } & \text { 235, }\end{array}$ doi:10.1016/J.PSYCHRES.2015.12.002

Han, S., \& Kahn, J. H. (2017). Attachment, emotion regulation difficulties, and disordered eating among college women and men. Counseling Psychologist, 45(8), 1066-1090. doi:10.1177/0011000017744884

Harper-Giuffre, H., \& MacKenzie, K. R. (1992). Group psychotherapy for eating disorders. In H. Harper-Giuffre \& K. R. MacKenzie (Eds.), Developing a Healthy Relationship with Your Body (pp. 329-333). Washington, USA: American Psychiatric Press.

Havighurst, S. S., Wilson, K. R., Harley, A. E., Kehoe, C., Efron, D., \& Prior, M. R. (2013). 'Tuning into kids': Reducing young children's behavior problems using an emotion coaching parenting program. Child Psychiatry and Human Development, 44(2), 247-264. doi:10.1007/s10578-012-0322-1

Havighurst, S. S., Wilson, K. R., Harley, A. E., Prior, M. R., \& Kehoe, C. (2010). Tuning in to Kids: Improving emotion socialization practices in parents of 
preschool children-findings from a community trial. Journal of Child Psychology and Psychiatry and Allied Disciplines, 51(12), 1342-1350. doi:10.1111/j.14697610.2010.02303.x

Haviland, M. G., Louise Warren, W., \& Riggs, M. L. (2000). An Observer Scale to Measure Alexithymia. $\quad$ Psychosomatics, 41(5), 385-392. doi:10.1176/APPI.PSY.41.5.385

Hayes, A. F. (2013). Introduction to Mediation, Moderation and Conditional Process Analysis: A Regression-Based Approach. New York, USA: Guildford Press.

Hayes, S. C., \& Smith, S. X. (2005). Get Out of Your Mind and Into Your Life: the New Acceptance and Commitment Therapy. New Harbinger Publications. $\begin{array}{ll}\text { Retrieved from } & \text { from }\end{array}$ https://books.google.co.uk/books?hl=en\&lr=\&id=q65CHYyzjzUC\&oi=fnd\&pg $=$ PR3\&dq=Get+out+of + your+mind + and + into + your + life $\&$ ots $=m-$ otuGiq_6\&sig=OTs4kn3DnEeJLn1kTZeB-Se6QXA\#v=onepage\&q=Get out of your mind and into your life $\& \mathrm{f}=$ false

Haynes, C., Lee, M. D., \& Yeomans, M. R. (2003). Interactive effects of stress, dietary restraint, and disinhibition on appetite. Eating Behaviors, 4(4), 369-383. doi:10.1016/J.EATBEH.2003.07.005

Heatherton, T. F., \& Baumeister, R. F. (1991). Binge eating as escape from selfawareness. Psychological Bulletin, 110(1), 86.

Hellhammer, D., Kirschbaum, C., Kudielka, B. M., \& Hellhammer, D. H. (2007). Ten years of research with the Trier Social Stress Test-revisited. In E. Harmon-Jones \& P. Winkielman (Eds.), Social Neuroscience. New York, USA: Guildford Press.

Helmers, K. F., \& Mente, A. (1999). Alexithymia and health behaviors in healthy male volunteers. Journal of Psychosomatic Research, 47(6), 635-45. 
doi:10.1016/S0022-3999(99)00067-7

Hendryx, M. S., Haviland, M. G., \& Shaw, D. G. (1991). Dimensions of alexithymia and their relationships to anxiety and depression. Journal of Personality Assessment, 56(2), 227-237. doi:10.1207/s15327752jpa5602_4

Herbert, B. M., Blechert, J., Hautzinger, M., Matthias, E., \& Herbert, C. (2013). Intuitive eating is associated with interoceptive sensitivity. Effects on body mass index. Appetite, 70, 22-30. doi:10.1016/j.appet.2013.06.082

Herbert, B. M., Herbert, C., \& Pollatos, O. (2011). On the relationship between interoceptive awareness and alexithymia: Is interoceptive awareness related to emotional awareness? Journal of Personality, 79(5), 1149-1175. doi:10.1111/j.1467-6494.2011.00717.x

Herbert, B. M., Pollatos, O., \& Schandry, R. (2007). Interoceptive sensitivity and emotion processing: An EEG study. International Journal of Psychophysiology, 65(3), 214-227. doi:10.1016/J.IJPSYCHO.2007.04.007

Hewig, J., Hagemann, D., Seifert, J., Gollwitzer, M., Naumann, E., \& Bartussek, D. (2005). A revised film set for the induction of basic emotions. Cognition and Emotion, 19(7), 1095-1109. doi:10.1080/02699930541000084

Hewitt, P. L., Flett, G. L., Turnbull-Donovan, W., \& Mikail, S. F. (1991). The Multidimensional Perfectionism Scale: Reliability, validity, and psychometric properties in psychiatric samples. Psychological Assessment, 3(3), 464-468.

Higgs, S., \& Donohoe, J. E. (2011). Focusing on food during lunch enhances lunch memory and decreases later snack intake. Appetite, 57(1), 202-206. doi:10.1016/j.appet.2011.04.016

Hofmann, W., Jansen, A., Nederkoorn, C., Houben, K., Hofmann, W., Roefs, A., \& Jansen, A. (2014). Control yourself or just eat what you like? Weight gain over a 
year is predicted by an interactive effect of response inhibition and implicit preference for snack foods. Health Psychology, 29(June), 389-393. doi:10.1037/a0019921

Holliday, J., Uher, R., Landau, S., Collier, D., \& Treasure, J. (2006). Personality pathology among individuals with a lifetime history of anorexia nervosa. Journal of Personality Disorders, 20(4), 417-430. doi:10.1521/pedi.2006.20.4.417

Honkalampi, K., Hintikka, J., Laukkanen, E., \& Viinamäki, J. L. H. (2001). Alexithymia and Depression: A Prospective Study of Patients With Major Depressive Disorder. Psychosomatics, 42(3), 229-234. doi:10.1176/APPI.PSY.42.3.229

Honkalampi, K., Hintikka, J., Tanskanen, A., Lehtonen, J., \& Viinamäki, H. (2000). Depression is strongly associated with alexithymia in the general population. Journal of Psychosomatic Research, 48(1), 99-104. doi:10.1016/S00223999(99)00083-5

Hruby, A., Manson, J. E., Qi, L., Malik, V. S., Rimm, E. B., Sun, Q., ... Hu, F. B. (2016). Determinants and Consequences of Obesity. Public Health, 106, 1656 1662. doi:10.2105/AJPH.2016.303326

Hsieh, H.-F., \& Shannon, S. E. (2005). Three Approaches to Qualitative Content Analysis. Qualitative Health Research, 15(9), 1277-1288. doi:10.1177/1049732305276687

Hudson, J. I., Hiripi, E., Pope, H. G., Kessler, R. C., \& Kessler, R. C. (2007). The prevalence and correlates of eating disorders in the National Comorbidity Survey Replication. Biological $\quad$ Psychiatry, $\quad$ 61(3), $\quad 348-58$. doi:10.1016/j.biopsych.2006.03.040

Hunter, P. G., Schellenberg, E. G., \& Schimmack, U. (2010). Feelings and Perceptions 
of Happiness and Sadness Induced by Music: Similarities, Differences, and Mixed Emotions. Psychology of Aesthetics, Creativity, and the Arts, 4(1), 47-56. doi:10.1037/a0016873

Izard, C. E. (2007). Basic Emotions, Natural Kinds, Emotion Schemas, and a New Paradigm. Perspectives on Psychological Science, 2(3), 260-280. doi:10.1111/j.1745-6916.2007.00044.x

Jackson, T., Weiss, K. E., Lunquist, J. J., \& Soderlind, A. (2005). Sociotropy and perceptions of interpersonal relationships as predictors of eating disturbances among college women: Two prospective studies. Journal of Genetic Psychology, 166(3), 346-360. doi:10.3200/GNTP.166.3.346-360

Jallais, C., \& Gilet, A.-L. L. (2010). Inducing changes in arousal and valence: Comparison of two mood induction procedures. Behavior Research Methods, 42(1), 318-325. doi:10.3758/BRM.42.1.318

James, W. (1884). What is an Emotion? Mind, 9(34), 188-205. doi:10.2307/2246769

Jansen, A., Vanreyten, A., \& Balveren, T. van. (2008). Negative affect and cueinduced overeating in non-eating disordered obesity. Appetite, 51, 556-562. doi:10.1016/j.appet.2008.04.009

Jansen, W., van de Looij-Jansen, P. M., de Wilde, E. J., \& Brug, J. (2008). Feeling fat rather than being fat may be associated with psychological well-being in young Dutch adolescents. Journal of Adolescent Health, 42(2), 128-136. doi:10.1016/J.JADOHEALTH.2007.07.015

Jasinska, A. J., Yasuda, M., Burant, C. F., Gregor, N., Khatri, S., Sweet, M., \& Falk, E. B. (2012). Impulsivity and inhibitory control deficits are associated with unhealthy eating in young adults. Appetite, 59(3), 738-747. doi:10.1016/J.APPET.2012.08.001 
Jeffery, R. W. (1996). Bias in reported body weight as a function of education, occupation, health and weight concern. Addictive Behaviors, 21(2), 217-222. doi:10.1016/0306-4603(95)00050-X

Johnson, M. K., Foley, M. A., Suengas, A. G., \& Raye, C. L. (1988). Phenomenal Characteristics of Memories for Perceived and Imagined Autobiographical Events. Journal of Experimental Psychology: General, 117(4), 371-376. doi:10.1037/0096-3445.117.4.371

Johnson, M. W. (2012). An efficient operant choice procedure for assessing delay discounting in humans: Initial validation in cocaine-dependent and control individuals. Experimental and Clinical Psychopharmacology, 20(3), 191-204. doi:10.1037/a0027088

Johnson, M. W., \& Bickel, W. K. (2008). An algorithm for identifying nonsystematic delay discounting data. Experimental and Clinical Psychopharmacology, 16(3), $264-274$.

Jorm, A. F. (2000). Does old age reduce the risk of anxiety and depression? A review of epidemiological studies across the adult life span (English). Psychological Medicine, 30(1), 11-22.

Jorm, A. F., Korten, A. E., Christensen, H., Jacomb, P. A., Rodgers, B., \& Parslow, P. A. (2003). Association of obesity with anxiety, depression and emotional wellbeing: a community survey. Australian \& New Zealand Journal of Public Health, 27(4), 434-440. doi:10.1111/j.1467-842X.2003.tb00423.x

Kahn, J. H., Tobin, R. M., Massey, A. E., \& Anderson, J. A. (2007). Measuring Emotional Expression with the Linguistic Inquiry and Word Count. The American Journal of Psychology, 120(2), 263. doi:10.2307/20445398

Kaiser, A. J., Milich, R., Lynam, D. R., \& Charnigo, R. J. (2012). Negative Urgency, 
Distress Tolerance, and substance abuse among college students. Addictive Behaviors, 37(10), 1075-1083. doi:10.1016/j.addbeh.2012.04.017

Kang, J. I., Namkoong, K., Yoo, S. W., Jhung, K., \& Kim, S. J. (2012). Abnormalities of emotional awareness and perception in patients with obsessive-compulsive disorder. Journal of Affective Disorders, 141(2-3), 286-293. doi:10.1016/J.JAD.2012.04.001

Karlsson, J., Persson, L.-O., Sjöström, L., \& Sullivan, M. (2000). Psychometric properties and factor structure of the Three-Factor Eating Questionnaire (TFEQ) in obese men and women. Results from the Swedish Obese Subjects (SOS) study. International Journal of Obesity, 24(12), 1715-1725. doi:10.1038/sj.ijo.0801442

Karukivi, M., Hautala, L., Kaleva, O., Haapasalo-Pesu, K.-M., Liuksila, P.-R., Joukamaa, M., \& Saarijärvi, S. (2010). Alexithymia is associated with anxiety among adolescents. Journal of Affective Disorders, 125(1), 383-387. doi:10.1016/j.jad.2010.02.126

Karukivi, M., Hautala, L., Korpelainen, J., Haapasalo-Pesu, K.-M., Liuksila, P.-R., Joukamaa, M., \& Saarijärvi, S. (2010). Alexithymia and eating disorder symptoms in adolescents. Eating Disorders, 18(3), 226-238. doi:10.1080/10640261003719518

Karyadi, K. A., \& King, K. M. (2011). Urgency and negative emotions: Evidence for moderation on negative alcohol consequences. Personality and Individual Differences, 51(5), 635-640. doi:10.1016/J.PAID.2011.05.030

Katterman, S. N., Kleinman, B. M., Hood, M. M., Nackers, L. M., \& Corsica, J. A. (2014). Mindfulness meditation as an intervention for binge eating, emotional eating, and weight loss: A systematic review. doi:10.1016/j.eatbeh.2014.01.005

Kearney, D. J., Milton, M. L., Malte, C. A., McDermott, K. A., Martinez, M., \& 
Simpson, T. L. (2012). Participation in mindfulness-based stress reduction is not associated with reductions in emotional eating or uncontrolled eating. Nutrition Research, 32(6), 413-420. doi:10.1016/J.NUTRES.2012.05.008

Keller, C., \& Siegrist, M. (2015). Does personality influence eating styles and food choices? Direct and indirect effects. Appetite, 84, 128-138. doi:10.1016/J.APPET.2014.10.003

Keltner, D., \& Kring, A. M. (1998). Emotion, social function, and psychopathology. Review of General Psychology, 2(3), 320-342. doi:10.1037/1089-2680.2.3.320

Kenardy, J., Butler, A., Carter, C., \& Moor, S. (2003). Eating, mood, and gender in a non-eatng disorder population, 4(2), 149-158. doi:10.1016/S14710153(03)00019-9

Kenealy, P. M. (1986). The velten mood induction procedure: A methodological review. Motivation and Emotion, 10(4), 315-335. doi:10.1007/BF00992107

Kent, S., Green, J., Reeves, G., Beral, V., Gray, A., Jebb, S. A., ... Sudlow, C. (2017). Hospital costs in relation to body-mass index in $1 \cdot 1$ million women in England: a prospective cohort study. The Lancet. Public Health, 2(5), e214-e222. doi:10.1016/S2468-2667(17)30062-2

Khalsa, S. S., \& Lapidus, R. C. (2016). Can Interoception Improve the Pragmatic Search for Biomarkers in Psychiatry? Frontiers in Psychiatry, 7, 121. doi:10.3389/fpsyt.2016.00121

Khalsa, S. S., Rudrauf, D., Damasio, A. R., Davidson, R. J., Lutz, A., \& Tranel, D. (2008). Interoceptive awareness in experienced meditators. Psychophysiology, 45(4), 671-677. doi:10.1111/j.1469-8986.2008.00666.x

Kirmayer, L. J., \& Robbins, J. M. (1993). Cognitive and social correlates of the Toronto Alexithymia Scale. Psychosomatics, 34(1), 41-52. doi:10.1016/S0033- 
3182(93)71926-X

Kirschbaum, C., Pirke, K. M., \& Hellhammer, D. H. (1993). The 'Trier social stress test' - A tool for investigating psychobiological stress responses in a laboratory setting. Neuropsychobiology, 28(1-2), 76-81. doi:10.1159/000119004

Kjelsås, E., Bjørnstrøm, C., \& Götestam, K. G. (2004). Prevalence of eating disorders in female and male adolescents (14-15 years). Eating Behaviors, 5(1), 13-25. doi:10.1016/S1471-0153(03)00057-6

Klabunde, M., Acheson, D. T., Boutelle, K. N., Matthews, S. C., \& Kaye, W. H. (2013). Interoceptive sensitivity deficits in women recovered from bulimia nervosa. $\quad$ Eating $\quad$ Behaviors, $\quad$ 14(4), $488-492$. doi:10.1016/J.EATBEH.2013.08.002

Kleiner, K. D., Gold, M. S., Frost-Pineda, K., Lenz-Brunsman, B., Perri, M. G., \& Jacobs, W. S. (2004). Body Mass Index and Alcohol Use, 23(3), 105-118. doi:10.1300/J069v23n03_08

Koball, A. M., Meers, M. R., Storfer-Isser, A., Domoff, S. E., \& Musher-Eizenman, D. R. (2012). Eating when bored: Revision of the Emotional Eating Scale with a focus on boredom. Health Psychology, 31(4), 521-524. doi:10.1037/a0025893

Kober, H. (2014). Emotion regulation in substance use disorders. In J. J. Gross (Ed.), Handbook of emotion regulation (2nd ed.). (pp. 428-446). New York, USA: Guildford Press.

Koenders, P. G., \& van Strien, T. (2011). Emotional eating, rather than lifestyle behavior, drives weight gain in a prospective study in 1562 employees. Journal of Occupational and Environmental Medicine, 53(11), 1287-1293. doi:10.1097/JOM.0b013e31823078a2

Koffarnus, M. N., Jarmolowicz, D. P., Mueller, E. T., \& Bickel, W. K. (2013). 
Changing delay discounting in the light of the competing neurobehavioral decision systems theory: A review. Journal of the Experimental Analysis of Behavior, 99(1), 32-57. doi:10.1002/jeab.2

Koh-Banerjee, P., Wang, Y., Hu, F. B., Spiegelman, D., Willett, W. C., \& Rimm, E. B. (2004). Changes in Body Weight and Body Fat Distribution as Risk Factors for Clinical Diabetes in US Men. American Journal of Epidemiology, 159(12), 1150-1159. doi:10.1093/aje/kwh167

Konttinen, H., Männistö, S., Sarlio-Lähteenkorva, S., Silventoinen, K., \& Haukkala, A. (2010). Emotional eating, depressive symptoms and self-reported food consumption. A population-based study. Appetite, 54(3), 473-479. doi:10.1016/J.APPET.2010.01.014

Kooiman, C. G., Spinhoven, P., \& Trijsburg, R. W. (2002). The assessment of alexithymia: A critical review of the literature and a psychometric study of the Toronto Alexithymia Scale-20. Journal of Psychosomatic Research, 53(6), 10831090. doi:10.1016/S0022-3999(02)00348-3

Kovacs, M., Sherrill, J., George, C. J., Pollock, M., Tumuluru, R. V., \& Ho, V. (2006). Contextual emotion-regulation therapy for childhood depression: Description and pilot testing of a new intervention. Journal of the American Academy of Child and Adolescent Pschiatry, $\quad$ 45(8), 892-903. doi:10.1097/01.chi.0000222878.74162.5a

Kreibig, S. D., Samson, A. C., \& Gross, J. J. (2013). The psychophysiology of mixed emotional states. Psychophysiology, 50(8), 799-811. doi:10.1111/psyp.12064

Kucharska-Pietura, K., Nikolaou, V., Masiak, M., \& Treasure, J. (2004). The recognition of emotion in the faces and voice of anorexia nervosa. International Journal of Eating Disorders, 35(1), 42-47. doi:10.1002/eat.10219 
Kuczmarski, M. F., Kuczmarski, R. J., \& Najjar, M. (2001). Effects of age on validity of self-reported height, weight, and body mass index: Findings from the third National Health and Nutrition Examination Survey, 1988-1994. Journal of the American Dietetic Association, 101(1), 28-34. doi:10.1016/S00028223(01)00008-6

Laghai, A., \& Joseph, S. (2000). Attitudes towards emotional expression: Factor structure, convergent validity and associations with personality. British Journal of Medical Psychology, 73(3), 381-384. doi:10.1348/000711200160598

Lahti-Koski, M., Pietinen, P., Heliovaara, M., \& Vartiainen, E. (2002). Associations of body mass index and obesity with physical activity, food choices, alcohol intake, and smoking in the 1982-1997 FINRISK studies. American Journal of Clinical Nutrition, 75, 809-817.

Laitinen, J., Ek, E., \& Sovio, U. (2002). Stress-related eating and drinking behavior and body mass index and predictors of this behavior. Preventive Medicine, 34(1), 29-39. doi:10.1006/pmed.2001.0948

Lane, R. D., Reiman, E. M., Ahern, G. L., Schwartz, G. E., \& Davidson, R. J. (1997). Neuroanatomical correlates of happiness, sadness, and disgust. American Journal of Psychiatry, 154(7), 926-933. doi:10.1176/ajp.154.7.926

Lane, R. D., Sechrest, L., Reidel, R., Weldon, V., Kaszniak, A., \& Schwartz, G. E. (1996). Impaired verbal and nonverbal emotion recognition in alexithymia. Psychosomatic Medicine, 58(3), 203-210.

Lane, R. D., Sechrest, L., \& Riedel, R. (1998). Sociodemographic correlates of alexithymia. Comprehensive Psychiatry, 39(6), 377-385. doi:10.1016/S0010440X(98)90051-7

Larsen, J. K., Brand, N., Bermond, B., \& Hijman, R. (2003). Cognitive and emotional 
characteristics of alexithymia: a review of neurobiological studies. Journal of Psychosomatic Research, 54(6), 533-541.

Larsen, J. K., van Strien, T., Eisinga, R., \& Engels, R. C. M. E. (2006). Gender differences in the association between alexithymia and emotional eating in obese individuals. Journal of Psychosomatic Research, 60(3), 237-243. doi:10.1016/J.JPSYCHORES.2005.07.006

Larsen, J. T., \& McGraw, A. (2014). The case for mixed emotions. Social and Personality Psychology Compass, 8(6), 263-274. doi:10.1111/spc3.12108

Larsen, J. T., \& McGraw, A. P. (2011). Further evidence for mixed emotions. Journal of Personality and Social Psychology, 100(6), 1095-1110. doi:10.1037/a0021846

Larsen, J. T., McGraw, A. P., \& Cacioppo, J. T. (2001). Can people be happy and sad at the same time.pdf. Journal of Personality and Social Psychology.

Lavie, C. J., Milani, R. V., \& Ventura, H. O. (2009). Obesity and Cardiovascular Disease. Journal of the American College of Cardiology, 53(21), 1925-1932. doi:10.1016/j.jacc.2008.12.068

Lazarevich, I., Irigoyen Camacho, M. E., Velázquez-Alva, M. del C., \& Zepeda Zepeda, M. (2016). Relationship among obesity, depression, and emotional eating in young adults. Appetite, 107, 639-644. doi:10.1016/j.appet.2016.09.011

Lehman, A. K., \& Rodin, J. (1989). Styles of self-nurturance and disordered eating. Journal of Consulting and Clinical Psychology, 57(1), 117-122. doi:10.1037/0022-006X.57.1.117

Leidy, N. K. (1994). Operationalizing Maslow's Theory: Development and Testing of the Basic Need Satisfaction Inventory. Issues in Mental Health Nursing, 15(3), 277-295. doi:10.3109/01612849409009390 
Leist, A., Ferring, D., \& Filipp, S.-H. (2010). Remembering positive and negative life events: Associations with future time perspective and functions of autobiographical memory. The Journal of Gerontopsychology and Geriatric Psychiatry, 23(3), 137-147. doi:10.1024/1662-9647/a000017

Levitsky, D. (2005). The non-regulation of food intake in humans: Hope for reversing the epidemic of obesity. Physiology \& Behavior, 86(5), 623-632. doi:10.1016/j.physbeh.2005.08.053

Linehan, M. (1993). Skills Training Manual for Treating Borderline Personality Disorder. New York, USA: Guildford Press.

Linehan, M. M. (1993). Cognitive-Behavioral Treatment of Borderline Personality Disorder. New York, USA: Guilford Press.

Linehan, M. M. (1995). Understanding Borderline Personality Disorder. New York, USA: Guildford Press.

Lluch, A., Herbeth, B., Méjean, L., \& Siest, G. (2000). Dietary intakes, eating style and overweight in the Stanislas Family Study. International Journal of Obesity, 24(11), 1493-1499. doi:10.1038/sj.ijo.0801425

Logan, G. D., Schachar, R. J., \& Tannock, R. (1997). Impulsivity and inhibitory control. Psychological Science, $\quad 8(1), \quad 60-64$. doi:10.1111/j.14679280.1997.tb00545.x

Longarzo, M., D’Olimpio, F., Chiavazzo, A., Santangelo, G., Trojano, L., \& Grossi, D. (2015). The relationships between interoception and alexithymic trait. The Self-Awareness Questionnaire in healthy subjects. Frontiers in Psychology, 6, 1149. doi:10.3389/fpsyg.2015.01149

Lorr, M., \& McNair, D. M. (1982). Manual for the Profile of Mood States. Bipolar Form (POMS-BI). San Diego, USA: Educational and Industrial Testing Service. 
Lorr, M., McNair, D. M., \& Fisher, S. (1982). Evidence for bipolar mood states. Journal of Personality Assessment, 46(4), 432-436.

Lovibond, P. F., \& Lovibond, S. H. (1995). The structure of negative emotional states: Comparison of the Depression Anxiety Stress Scales (DASS) with the Beck Depression and Anxiety Inventories. Behaviour Research and Therapy, 33(3), 335-343. doi:10.1016/0005-7967(94)00075-U

Lowe, M. R., \& Eldredge, K. L. (1993). The role of impulsiveness in normal and disordered eating. In W. G. McGowan, J. L. Johnson, \& M. B. Shure (Eds.), The Impulsive Client: theory, research and treatment (pp. 247-263). Washington, USA: American Psychological Association.

Lowe, M. R., \& Maycock, B. (1988). Restraint, disinhibition, hunger and negative affect eating. Addictive Behaviors, 13(4), 369-377. doi:10.1016/03064603(88)90043-3

Loxton, N. J., Dawe, S., \& Cahill, A. (2011). Does negative mood drive the urge to eat? The contribution of negative mood, exposure to food cues and eating style. Appetite, 56(2), 368-374. doi:10.1016/J.APPET.2011.01.011

Lukasiewicz, E., Mennen, L. I., Bertrais, S., Arnault, N., Preziosi, P., Galan, P., \& Hercberg, S. (2005). Alcohol intake in relation to body mass index and waist-tohip ratio: the importance of type of alcoholic beverage. Public Health Nutrition, 8(03), 315-320. doi:10.1079/PHN2004680

Luminet, O., Bagby, R. M., Wagner, H., Taylor, G. J., \& Parker, J. D. A. (1999). Relation between Alexithymia and the five-factor model of personality: A facetlevel analysis. Journal of Personality Assessment, 73(3), 345-358. doi:10.1207/S15327752JPA7303_4

Luminet, O., Zech, E., Rimé, B., \& Wagner, H. (1998). How to predict confrontation 
with emotional episodes? The respective impact of emotion intensity, the five factor model and alexithymia. submitted, University of Louvain. Louvain-laNeuve, Belgium.

Lumley, M. A., Gustavson, B. J., Partridge, R. T., \& Labouvie-Vief, G. (2005). Assessing alexithymia and related emotional ability constructs using multiple methods: Interrelationships among measures. Emotion, 5(3), 329-342. doi:10.1037/1528-3542.5.3.329

Lumley, M. A., Neely, L. C., \& Burger, A. J. (2007, November 14). The assessment of alexithymia in medical settings: Implications for understanding and treating health problems. Journal of Personality Assessment. Taylor \& Francis Group. doi:10.1080/00223890701629698

Lumley, M. A., Ovies, T., Stettner, L., Wehmer, F., \& Lakey, B. (1996). Alexithymia, social support and health problems. Journal of Psychosomatic Research, 41(6), $519-530$.

Lundh, L.-G., Johnsson, A., Sundqvist, K., \& Olsson, H. (2002). Alexithymia, memory of emotion, emotional awareness, and perfectionism. Emotion, 2(4), 361-379. doi:10.1037/1528-3542.2.4.361

Luppino, F. S., de Wit, L. M., Bouvy, P. F., Stijnen, T., Cuijpers, P., Penninx, B. W. J. H., \& Zitman, F. G. (2010). Overweight, Obesity, and Depression. Archives of General Psychiatry, 67(3), 220. doi:10.1001/archgenpsychiatry.2010.2

Lyman, B. (1982). The Nutritional Values and Food Group Characteristics of Foods Preferred During Various Emotions. The Journal of Psychology, 112(1), 121127. doi:10.1080/00223980.1982.9923544

Macht, M. (1999). Characteristics of eating in anger, fear, sadness and joy. Appetite, 33(1), 129-139. doi:10.1006/APPE.1999.0236 
Macht, M., \& Dettmer, D. (2006). Everyday mood and emotions after eating a chocolate bar or an apple. Appetite, 46(3), 332-336. doi:10.1016/j.appet.2006.01.014

Macht, M., Gerer, J., \& Ellgring, H. (2003). Emotions in overweight and normalweight women immediately after eating foods differing in energy. Physiology and Behavior, 80(2-3), 367-374. doi:10.1016/j.physbeh.2003.08.012

Macht, M., \& Mueller, J. (2007). Immediate effects of chocolate on experimentally induced $\quad$ states. $\quad$ Appetite, 49(3), 667-674. doi:10.1016/J.APPET.2007.05.004

Macht, M., Roth, S., \& Ellgring, H. (2002). Chocolate eating in healthy men during experimentally induced sadness and joy. Appetite, 39, 147-158. doi:10.1006/APPE.2002.0499

Macht, M., \& Simons, G. (2000). Emotions and eating in everyday life. Appetite, 35(1), 65-71. doi:10.1006/appe.2000.0325

Maister, L., \& Tsakiris, M. (2014). My face, my heart: Cultural differences in integrated bodily self-awareness. Cognitive Neuroscience, 5(1), 10-16. doi:10.1080/17588928.2013.808613

Manwaring, J. L., Green, L., Myerson, J., Strube, M. J., \& Wilfley, D. E. (2011). Discounting of Various types of rewards by women with and without binge eating Disorder: Evidence for general rather than specific Differences. The Psychological Record, 61(4), 561-582. doi:24039301

Marchesi, C., Brusamonti, E., \& Maggini, C. (2000). Are alexithymia, depression, and anxiety distinct constructs in affective disorders? Journal of Psychosomatic Research, 49(1), 43-49. doi:10.1016/S0022-3999(00)00084-2

Marroquín, B. (2011). Interpersonal emotion regulation as a mechanism of social 
support in depression. Clinical Psychology Review, 31(8), 1276-1290. doi:10.1016/j.cpr.2011.09.005

Mattila, A. K., Poutanen, O., Koivisto, A.-M., Salokangas, R. K. R., \& Joukamaa, M. (2008). The performance of diagnostic measures of depression in alexithymic and nonalexithymic subjects. General Hospital Psychiatry, 30(1), 77-79. doi:10.1016/J.GENHOSPPSYCH.2007.08.011

Mattila, A. K., Salminen, J. K., Nummi, T., \& Joukamaa, M. (2006). Age is strongly associated with alexithymia in the general population. Journal of Psychosomatic Research, 61(5), 629-635. doi:10.1016/J.JPSYCHORES.2006.04.013

Maunder, R. G., \& Hunter, J. J. (2001). Attachement and psychosomatic medicine: Development contributions to stress and disease. Psychosomatic Medicine, 63(4), $556-567$.

Maxwell, S. E., \& Delaney, H. D. (1993). Bivariate median splits and spurious statistical significance. Psychological Bulletin, 113(1), 181-190. doi:10.1037/0033-2909.113.1.181

Mazur, J. E., Stellar, J. R., \& Waraczynski, M. (1987). Self-control choice with electrical stimulation of the brain as a reinforcer. Behavioural Processes, 15(23), 143-153. doi:10.1016/0376-6357(87)90003-9

McCrae, R. R., Costa, P. T., de Lima, M. P., Simoes, A., Ostendorf, F., Angleitner, A., ... Piedmont, R. L. (1999). Age differences in personality across the adult life span: Parallels in five cultures. Developmental Psychology, 35(2), 466-477. doi:10.1103/PhysRevB.66.085421

McFarlane, T., Urbszat, D., \& Olmsted, M. P. (2011). "I Feel Fat”: An experimental induction of body displacement in disordered eating. Behaviour Research and Therapy, 49(4), 289-293. doi:10.1016/J.BRAT.2011.01.008 
McGinnis, D., \& Roberts, P. (1996). Qualitative characteristics of vivid memories attributed to real and imagined experiences. The American Journal of Psychology, 109(1), 59. doi:10.2307/1422927

McHugh, L., \& Wood, R. L. (2008). Using a temporal discounting paradigm to measure decision-making and impulsivity following traumatic brain injury: A pilot study. Brain Injury, 22(9), 715-721. doi:10.1080/02699050802263027

McKerchar, T. L., Green, L., \& Myerson, J. (2010). On the scaling interpretation of exponents in hyperboloid models of delay and probability discounting. Behavioural Processes, 84(1), 440-444. doi:10.1016/J.BEPROC.2010.01.003

McLean, C. P., \& Anderson, E. R. (2009). Brave men and timid women? A review of the gender differences in fear and anxiety. Clinical Psychology Review, 29(6), 496-505. doi:10.1016/j.cpr.2009.05.003

McNair, D. M., Lorr, M., \& Droppleman, L. F. (1971). Manual: Profile of Mood States. San Diego, USA: Educational and Industrial Testing Service.

McNair, D. M., Lorr, M., \& Droppleman, L. F. (1992). Manual: Profile of Mood States. San Diego, USA: Educational and Industrial Testing Service.

Medley, A. N., Capron, D. W., Korte, K. J., \& Schmidt, N. B. (2013). Anxiety sensitivity: A potential vulnerability factor for compulsive hoarding. Cognitive Behaviour Therapy, 42(1), 45-55. doi:10.1080/16506073.2012.738242

Meganck, R., Vanheule, S., Desmet, M., \& Inslegers, R. (2010). The Observer Alexithymia Scale: A reliable and valid alternative for alexithymia measurement? Journal of Personality Assessment, 92(2), 175-185.

Mehling, W. E., Price, C., Daubenmier, J. J., Acree, M., Bartmess, E., Stewart, A., ... Baer, R. (2012). The Multidimensional Assessment of Interoceptive Awareness (MAIA). PLoS ONE, 7(11), e48230. doi:10.1371/journal.pone.0048230 
Mehrabian, A. (1980). Basic dimensions for a general psychological theory: implications for personality, social, environmental, and developmental studies. Cambridge, USA: Oelgeschlager, Gunn \& Hain.

Mellis, A. M., Woodford, A. E., Stein, J. S., \& Bickel, W. K. (2017). A second type of magnitude effect: Reinforcer magnitude differentiates delay discounting between substance users and controls. Journal of the Experimental Analysis of Behavior, 107(1), 151-160. doi:10.1002/jeab.235

Melloni, M., Sedeño, L., Couto, B., Reynoso, M., Gelormini, C., Favaloro, R., ... Ibanez, A. (2013). Preliminary evidence about the effects of meditation on interoceptive sensitivity and social cognition. Behavioral and Brain Functions, 9(1), 47. doi:10.1186/1744-9081-9-47

Menary, K. R., Corbin, W. R., Leeman, R. F., Fucito, L. M., Toll, B. A., DeMartini, K., \& O’Malley, S. S. (2015). Interactive and Indirect Effects of Anxiety and Negative Urgency on Alcohol-Related Problems. Alcoholism, Clinical and Experimental Research, 39(7), 1267-74. doi:10.1111/acer.12762

Michaud, C., Kahn, J. P., Musse, N., Burlet, C., Nicolas, J. P., \& Mejean, L. (1990). Relationships between a critical life event and eating behaviour in high-school students. Stress Medicine, 6(1), 57-64. doi:10.1002/smi.2460060112

Mikolajczak, M., \& Luminet, O. (2006). Is alexithymia affected by situational stress or is it a stable trait related to emotion regulation? Personality and Individual Differences, 40(7), 1399-1408. doi:10.1016/J.PAID.2005.10.020

Mills, C., \& D'Mello, S. (2014). On the Validity of the Autobiographical Emotional Memory Task for Emotion Induction. PLoS ONE, 9(4), e95837. doi:10.1371/journal.pone.0095837

Mirowsky, J., \& Ross, C. E. (1995). Sex Differences in Distress: Real or Artifact? 
American Sociological Review, 60(3), 449. doi:10.2307/2096424

Moeller, F. G., Barratt, E. S., Dougherty, D. M., Schmitz, J. M., \& Swann, A. C. (2001). Psychiatric Aspects of Impulsivity. American Journal of Psychiatry, 158(11), 1783-1793. doi:10.1176/appi.ajp.158.11.1783

Moghaddam, M. V., \& BabaKhani, N. (2016). The comparison of emotional selfregulation and problem solving ability relationship with emotional overeating in obese and normal women. Journal of Psychology \& Behavioral Studies, 4(SP1), $25-38$.

Mohammed, W. A., Muthumayandi, K., \& Sharma, D. (2018). The effect of mindfulness meditation on therapists' body-awareness and burnout in different forms of practice. doi:10.1080/21679169.2018.1452980

Monterosso, J., \& Ainslie, G. (1999). Beyond discounting: possible experimental models of impulse control. Psychopharmacology, 146(4), 339-347. doi:10.1007/PL00005480

Moon, A., \& Berenbaum, H. (2009). Emotional awareness and emotional eating. Cognition and Emotion, 23(3), 417-429. doi:10.1080/02699930801961798

Morgan, J. F., Reid, F., \& Lacey, J. H. (1999). The SCOFF questionnaire: assessment of a new screening tool for eating disorders. BMJ (Clinical Research Ed.), 319(7223), 1467-8. doi:10.1136/BMJ.319.7223.1467

Morie, K. P., \& Ridout, N. (2018). Alexithymia and maladaptive regulatory behaviours in substance use disorders and eating disorders. In O. Luminet, R. M. Bagby, \& G. J. Taylor (Eds.), Alexithymia: Advances in Research, Theory and Clinical Practice (pp. 158-173). Cambridge University Press.

Morrow, J., \& Nolen-Hoeksema, S. (1990). Effects of responses to depression on the remediation of depressive affect. Journal of Personality and Social Psychology, 
58(3), 519-527. doi:10.1037/0022-3514.58.3.519

Munsch, S., Michael, T., Biedert, E., Meyer, A. H., \& Margraf, J. (2008). Negative mood induction and unbalanced nutrition style as possible triggers of binges in binge eating disorder (BED). Eating and Weight Disorders, 13(1), 22-29. doi:10.1007/BF03327781

Murphy, C. M., Stojek, M. K., \& MacKillop, J. (2014). Interrelationships among impulsive personality traits, food addiction, and Body Mass Index. Appetite, 73, 45-50. doi:10.1016/j.appet.2013.10.008

Murphy, J., Brewer, R., Catmur, C., \& Bird, G. (2017). Interoception and psychopathology: A developmental neuroscience perspective. Developmental Cognitive Neuroscience, 23, 45-56. doi:10.1016/j.dcn.2016.12.006

Murphy, J., Catmur, C., \& Bird, G. (2018). Alexithymia is associated with a multidomain, multidimensional failure of interoception: Evidence from novel tests. Journal of Experimental Psychology: General, 147(3), 398-408. doi:10.1037/xge0000366

Nasser, J. A., Gluck, M. E., \& Geliebter, A. (2004). Impulsivity and test meal intake in obese binge eating women. Appetite, 43(3), 303-307. doi:10.1016/J.APPET.2004.04.006

Nederkoorn, C., Smulders, F. T. Y., Havermans, R. C., Roefs, A., \& Jansen, A. (2006). Impulsivity in obese women. Appetite, 47(2), 253-256.

Newman, E., O’Connor, D. B., \& Conner, M. (2007). Daily hassles and eating behaviour: The role of cortisol reactivity status. Psychoneuroendocrinology, 32(2), 125-132. doi:10.1016/J.PSYNEUEN.2006.11.006

Ng, M., Fleming, T., Robinson, M., Thomson, B., Graetz, N., Margono, C., ... Gakidou, E. (2014). Global, regional, and national prevalence of overweight and 
obesity in children and adults during 1980-2013: A systematic analysis for the Global Burden of Disease Study 2013. The Lancet, 384(9945), 766-781. doi:10.1016/S0140-6736(14)60460-8

Nguyen-Michel, S. T., Unger, J. B., \& Spruijt-Metz, D. (2007). Dietary correlates of emotional eating in adolescence. Appetite, 49(2), 494-9. doi:10.1016/j.appet.2007.03.005

Nguyen-Rodriguez, S. T., Chou, C.-P., Unger, J. B., \& Spruijt-Metz, D. (2008). BMI as a moderator of perceived stress and emotional eating in adolescents. Eating Behaviors, 9(2), 238-246. doi:10.1016/J.EATBEH.2007.09.001

Nguyen-Rodriguez, S. T., Unger, J. B., \& Spruijt-Metz, D. (2009). Psychological determinants of emotional eating in adolescence. Eating Disorders, 17(3), 21124. doi:10.1080/10640260902848543

Noli, G., Cornicelli, M., Marinari, G. M., Carlini, F., Scopinaro, N., \& Adami, G. F. (2010). Alexithymia and eating behaviour in severely obese patients. Journal of Human Nutrition and Dietetics, 23(6), 616-619. doi:10.1111/j.1365277X.2010.01079.x

Norton, G. N. M., Anderson, A. S., \& Hetherington, M. M. (2006). Volume and variety: Relative effects on food intake. Physiology \& Behavior, 87(4), 714-722. doi:10.1016/J.PHYSBEH.2006.01.010

Nowakowski, M. E., McFarlane, T., \& Cassin, S. (2013). Alexithymia and eating disorders: a critical review of the literature. J. Eat. Disord, 1(21), 10.1186.

O’Reilly, G. A., Cook, L., Spruijt-Metz, D., \& Black, D. S. (2014). Mindfulness-based interventions for obesity-related eating behaviours: a literature review. Obesity Reviews : An Official Journal of the International Association for the Study of Obesity, 15(6), 453-61. doi:10.1111/obr.12156 
OCED. (2017). Obesity Update 2017. Retrieved from www.oecd.org/health/obesityupdate.htm

Odum, A. L. (2011). Delay discounting: I'm a k, you're a k. Journal of the Experimental Analysis of Behavior, 96(3), 427-39. doi:10.1901/jeab.2011.96423

Ogden, C. L., Carroll, M. D., Lawman, H. G., Fryar, C. D., Kruszon-Moran, D., Kit, B. K., \& Flegal, K. M. (2016, June 7). Trends in obesity prevalence among children and adolescents in the United States, 1988-1994 through 2013-2014. JAMA - Journal of the American Medical Association. American Medical Association. doi:10.1001/jama.2016.6361

Oliver, G., \& Wardle, J. (1999). Perceived Effects of Stress on Food Choice. Physiology \& Behavior, 66(3), 511-515. doi:10.1016/S0031-9384(98)00322-9

Oliver, G., Wardle, J., \& Gibson, L. (2000). Stress and food choice: A laboratory study. Psychosomatic Medicine, 62(6), 853-865.

Ouwens, M. A., van Strien, T., \& van Leeuwe, J. F. J. (2009). Possible pathways between depression, emotional and external eating. A structural equation model. Appetite, 53(2), 245-248. doi:10.1016/J.APPET.2009.06.001

Ouwens, M. A., van Strien, T., van Leeuwe, J. F. J., \& van der Staak, C. P. F. (2009). The dual pathway model of overeating. Replication and extension with actual food consumption. Appetite, 52, 234-237. doi:10.1016/j.appet.2008.07.010

Paans, N. P. G., Bot, M., Brouwer, I. A., Visser, M., Roca, M., Kohls, E., ... Penninx, B. W. J. H. (2018). The association between depression and eating styles in four European countries: The MooDFOOD prevention study. Journal of Psychosomatic Research, 108, 85-92. doi:10.1016/j.jpsychores.2018.03.003 Panayiotou, G., Leonidou, C., Constantinou, E., Hart, J., Rinehart, K. L., Sy, J. T., \& 
Björgvinsson, T. (2015). Do alexithymic individuals avoid their feelings? Experiential avoidance mediates the association between alexithymia, psychosomatic, and depressive symptoms in a community and a clinical sample. Comprehensive Psychiatry, 56, 206-216. doi:10.1016/j.comppsych.2014.09.006

Parker, J. D. A., Bagby, R. M., \& Taylor, G. J. (1991). Alexithymia and depression: Distinct or overlapping constructs? Comprehensive Psychiatry, 32(5), 387-394. doi:10.1016/0010-440X(91)90015-5

Parker, J. D. A., \& Taylor, G. J. (1997). Relations between alexithymia, personality and affects. In G. J. Taylor, R. M. Bagby, \& J. D. A. Parker (Eds.), Disorders of Affect Regulation: Alexithymia in Medical Illness and Psychiatric Illness (pp. 6792). Cambridge, UK: Cambridge University Press.

Parker, J. D. A., Taylor, G. J., \& Bagby, R. M. (1989). The alexithymia construct: Relationship with sociodemographic variables and intelligence. Comprehensive Psychiatry, 30(5), 434-441. doi:10.1016/0010-440X(89)90009-6

Parker, J. D. A., Taylor, G. J., \& Bagby, R. M. (1998). Alexithymia: relationship with ego defense and coping styles. Comprehensive Psychiatry, 39(2), 91-98.

Parker, J. D. A., Taylor, G. J., \& Bagby, R. M. (2001). The relationship between emotional intelligence and alexithymia. Personality and Individual Differences, 30(1), 107-115. doi:10.1016/S0191-8869(00)00014-3

Parker, J. D. A., Taylor, G. J., \& Bagby, R. M. (2003). The 20-Item Toronto Alexithymia Scale III. Reliability and factorial validity in a community population. Journal of Psychosomatic Research, 55(3), 269-275. doi:10.1016/S0022-3999(02)00578-0

Parkin, L., Morgan, R., Rosselli, A., Howard, M., Sheppard, A., Evans, D., ... Dunn, B. (2014). Exploring the Relationship Between Mindfulness and Cardiac 
Perception. Mindfulness, 5(3), 298-313. doi:10.1007/s12671-012-0181-7

Patterson, C. M., \& Newman, J. P. (1993). Reflectivity and learning from aversive events: Toward a psychological mechanism for the syndromes of disinhibition. Psychological Review, 100(4), 716-736. doi:10.1037/0033-295X.100.4.716

Patton, J. H., Stanford, M. S., \& Barratt, E. S. (1995). Factor structure of the barratt impulsiveness scale. Journal of Clinical Psychology, 51(6), 768-774. doi:10.1002/1097-4679(199511)51:6<768::AID-JCLP2270510607>3.0.CO;2-1

Paxton, S. J., \& Phythian, K. (1999). Body Image, Self-Esteem, and Health Status in Middle and Later Adulthood. Australian Psychologist, 34(2), 116-121. doi:10.1080/00050069908257439

Péneau, S., Ménard, E., Méjean, C., Bellisle, F., \& Hercberg, S. (2013). Sex and dieting modify the association between emotional eating and weight status. The American Journal of Clinical Nutrition, 97(6), 1307-1313. doi:10.3945/ajcn.112.054916

Pennebaker, J. W., Boyd, R. L., Jordan, K., \& Blackburn, K. (2015). The Development and Psychometric Properties of LIWC2015.

Pennebaker, J. W., Chung, C. K., Ireland, M., Gonzales, A., \& Booth, R. J. (2007). The Development and Psychometric Properties of LIWC 2007. LIWC.net.

Pennebaker, J. W., Francis, M. E., \& Booth, R. J. (2001). Linguistic Inquiry and Word Count: LIWC 2001. Mahway: Lawrence Erlbaum Associates 71.

Peters, J., Miedl, S. F., \& Büchel, C. (2012). Formal comparison of dual-parameter temporal discounting models in controls and pathological gamblers. PLOS ONE, 7(11), e47225. doi:10.1371/journal.pone.0047225

Petit, G., Luminet, O., Maurage, F., Tecco, J., Lechantre, S., Ferauge, M., ... de Timary, P. (2015). Emotion Regulation in Alcohol Dependence. Alcoholism: 
Clinical and Experimental Research, 39(12), 2471-2479. doi:10.1111/acer.12914

Piccinelli, M., \& Wilkinson, G. (2000). Gender differences in depression: Critical review. British Journal of Psychiatry, 177(06), 486-492. doi:10.1192/bjp.177.6.486

Pike, C. (2013). The Association between alexithymia, impulsivity and negative affect in emotional and external eating. University of Canterbury. Retrieved from http://hdl.handle.net/10092/8986

Pinaquy, S., Chabrol, H., Simon, C., Louvet, J.-P., \& Barbe, P. (2003). Emotional Eating, Alexithymia, and Binge-Eating Disorder in Obese Women. Obesity Research, 11(2), 195-201. doi:10.1038/oby.2003.31

Polivy, J., \& Herman, C. P. (1999). Distress and eating: Why do dieters overeat? International Journal of Eating Disorders, 26(2), 153-164. doi:10.1002/(SICI)1098-108X(199909)26:2<153::AID-EAT4>3.0.CO;2-R

Pollatos, O., Herbert, B. M., Matthias, E., \& Schandry, R. (2007). Heart rate response after emotional picture presentation is modulated by interoceptive awareness. International Journal of Psychophysiology, 63(1), 117-124. doi:10.1016/J.IJPSYCHO.2006.09.003

Pollatos, O., Kurz, A.-L., Albrecht, J., Schreder, T., Kleemann, A. M., Schöpf, V., ... Schandry, R. (2008). Reduced perception of bodily signals in anorexia nervosa. Eating Behaviors, 9(4), 381-388. doi:10.1016/J.EATBEH.2008.02.001

Pombo, S., Félix da Costa, N., Ismail, F., Cardoso, J. M. N., \& Figueira, M. L. (2015). Alexithymia and alcohol dependence: Do different subtypes manifest different emotion regulations? Addiction Research \& Theory, 23(3), 187-195.

Potter, W. J., \& Levine-Donnerstein, D. (1999). Rethinking validity and reliability in 
content analysis. Journal of Applied Communication Research, 27(3), 258-284. doi:10.1080/00909889909365539

Price, M. (2016). Sweet Temptation: The Role of Impulsivity in Overeating, Overweight and Obesity. Swansea University.

Price, M., Higgs, S., \& Lee, M. (2015). Self-reported eating traits: Underlying components of food responsivity and dietary restriction are positively related to BMI. Appetite, 95, 203-210. doi:10.1016/J.APPET.2015.07.006

Price, M., Higgs, S., Maw, J., \& Lee, M. (2016). A dual-process approach to exploring the role of delay discounting in obesity. Physiology \& Behavior, 162, 46-51.

Price, M., Lee, M., \& Higgs, S. (2016). Food-specific response inhibition, dietary restraint and snack intake in lean and overweight/obese adults: a moderatedmediation model. International Journal of Obesity, 40(5), 877-882. doi:10.1038/ijo.2015.235

Prince, J. D., \& Berenbaum, H. (1993). Alexithymia and hedonic capacity. Journal of Research in Personality, 27(1), 15-22. doi:10.1006/jrpe.1993.1002

Rachlin, H. (1995). Self-control: Beyond commitment. Behavioral and Brain Sciences, 18(01), 109. doi:10.1017/S0140525X00037602

Racine, S. E., Keel, P. K., Burt, S. A., Sisk, C. L., Neale, M., Boker, S., \& Klump, K. L. (2013). Exploring the relationship between negative urgency and dysregulated eating: Etiologic associations and the role of negative affect. Journal of Abnormal Psychology, 122(2), 433-44. doi:10.1037/a0031250

Rasmussen, E. B., Lawyer, S. R., \& Reilly, W. (2010). Percent body fat is related to delay and probability discounting for food in humans. Behavioural Processes, 83(1), 23-30. doi:10.1016/j.beproc.2009.09.001

Read, D., \& Read, N. L. (2004). Time discounting over the lifespan. Organizational 
Behavior and Human Decision Processes, 94(1), 22-32. doi:10.1016/j.obhdp.2004.01.002

Reaves, D. L., Christiansen, P., Boyland, E., Halford, J. C. G., Llewellyn, C. H., \& Hardman, C. A. (2016). Cross-sectional associations between personality, eating to cope, and consumption. Appetite, 107(107), 689-690. doi:10.1016/j.appet.2016.08.076

Reiss, S., \& McNally, R. J. (1985). The expectancy model of fear. In S. Reiss \& R. R. Boorzin (Eds.), Theoretcial Issues in Behaviour Therapy (pp. 107-122). New York, USA: Academic Press.

Ricca, V., Castellini, G., Fioravanti, G., Lo Sauro, C., Rotella, F., Ravaldi, C., ... Faravelli, C. (2012). Emotional eating in anorexia nervosa and bulimia nervosa. Comprehensive Psychiatry, 53(3), 245-251. doi:10.1016/j.comppsych.2011.04.062

Richards, J. B., Zhang, L., Mitchell, S. H., \& de Wit, H. (1999). Delay or probability discounting in a model of impulsive behavior: effect of alcohol. Journal of the Experimental Analysis of Behavior, 71(2), 121-143. doi:10.1901/jeab.1999.71121

Ridout, N., Thom, C., \& Wallis, D. J. (2010). Emotion recognition and alexithymia in females with non-clinical disordered eating. Eating Behaviors, 11(1), 1-5. doi:10.1016/J.EATBEH.2009.07.008

Ridout, N., Wallis, D. J., Autwal, Y., Kralj, A., Sellis, J., \& Taylor, E. (2011). The influence of alexithymia and mood on eating psychopathology in a non-clinical female sample. In K. M. Appleton \& S. Higgs (Eds.), British Feeding and Drinking Group (pp. 553-569). doi:10.1016/j.appet.2011.05.091

Ridout, N., Wallis, D. J., Autwal, Y., \& Sellis, J. (2012). The influence of emotional 
intensity on facial emotion recognition in disordered eating. Appetite, 59(1), 181186. doi:10.1016/J.APPET.2012.04.013

Rief, W., Heuser, J., \& Fichter, M. M. (1996). What does the Toronto alexithymia scale TAS-R measure? Journal of Clinical Psychology, 52(4), 423-429. doi:10.1002/(SICI)1097-4679(199607)52:4<423::AID-JCLP6>3.0.CO;2-Q

Robbins, T. W., \& Fray, P. J. (1980). Stress-induced eating: Fact, fiction or misunderstanding? Appetite, 1(2), 103-133. doi:10.1016/S0195-6663(80)800158

Robinson, A. L., Kosmerly, S., Mansfield-Green, S., \& Lafrance, G. (2014). Disordered eating behaviours in an undergraduate sample: Associations among gender, body mass index, and difficulties in emotion regulation. Canadian Journal of Behavioural Science, 46(3), 320-326. doi:10.1037/a0031123

Robinson, E., Haynes, A., Hardman, C. A., Kemps, E., Higgs, S., \& Jones, A. (2017). The bogus taste test: Validity as a measure of laboratory food intake. Appetite, 116, 223-231. doi:10.1016/J.APPET.2017.05.002

Rokholm, B., Baker, J. L., \& Sørensen, T. I. A. (2010). The levelling off of the obesity epidemic since the year 1999 - a review of evidence and perspectives. Obesity Reviews, 11(12), 835-846. doi:10.1111/j.1467-789X.2010.00810.x

Roosen, M. A., Safer, D., Adler, S., Cebolla, A., \& van Strien, T. (2012). Group dialectical behavior therapy adapted for obese emotional eaters: A pilot study. Nutrición Hospitalaria, 27(4), 1141-1147. doi:10.3305/nh.2012.27.4.5843

Rosenbaum, D. L., \& White, K. S. (2015). The relation of anxiety, depression, and stress to binge eating behavior. Journal of Health Psychology, 20(6), 887-898. doi:10.1177/1359105315580212

Royal, J., \& Kurtz, J. (2010). I ate what?! The effect of stress and dispositional eating 
style on food intake and behavioral awareness. Personality and Individual Differences, 49(6), 565-569. doi:10.1016/j.paid.2010.04.022

Rubin, D. C., \& Berntsen, D. (2003). Life scripts help to maintain autobiographical memories of highly positive, but not highly negative, events. Memory \& Cognition, 31(1), 1-14.

Rude, S., \& McCarthy, C. (2003). Emotional functioning in depressed and depressionvulnerable college students. Cognition \& Emotion, 17(5), 799-806. doi:10.1080/02699930302283

Ruth, S., \& Padmakumari, P. (2014). Recent Trends in Alexithymia. International Journal of Psychology and Behavioral Sciences, 4(3), 106-111.

Saarijärvi, S., Salminen, J. ., \& Toikka, T. . (2001). Alexithymia and depression: A 1year follow-up study in outpatients with major depression. Journal of Psychosomatic Research, 51(6), 729-733. doi:10.1016/S0022-3999(01)00257-4

Saelens, B. E., \& Epstein, L. H. (1996). Reinforcing value of food in obese and nonobese women. Appetite, 27(1), 41-50. doi:10.1006/appe.1996.0032

Safer, D. L., Robinson, A. H., \& Jo, B. (2010). Outcome from a randomized controlled trial of group therapy for binge eating disorder: comparing dialectical behavior therapy adapted for binge eating to an active comparison group therapy. Behavior Therapy, 41(1), 106-20. doi:10.1016/j.beth.2009.01.006

Safer, D. L., Telch, C. F., \& Agras, W. S. (2001). Dialectical Behavior Therapy for Bulimia Nervosa. American Journal of Psychiatry, 158(4), 632-634. doi:10.1176/appi.ajp.158.4.632

Salas, C. E., Radovic, D., \& Turnbull, O. H. (2012). Inside-out: Comparing internally generated and externally generated basic emotions. Emotion, 12(3), 568-578. doi:10.1037/a0025811 
Salminen, J. J. K., Saarijärvi, S., Äärelä, E., Toikka, T., \& Kauhanen, J. (1999). Prevalence of alexithymia and its association with sociodemographic variables in the general population of finland. Journal of Psychosomatic Research, 46(1), 7582. doi:10.1016/S0022-3999(98)00053-1

Salovey, P., \& Mayer, J. D. (1990). Emotional Intelligence. Imagination, Cognition and Personality, 9(3), 185-211. doi:10.2190/DUGG-P24E-52WK-6CDG

Samson, A. C., Kreibig, S. D., Soderstrom, B., Wade, A. A., \& Gross, J. J. (2016). Eliciting positive, negative and mixed emotional states: A film library for affective scientists. Cognition and Emotion, 30(5), 827-856. doi:10.1080/02699931.2015.1031089

Sasai, K., Tanaka, K., \& Hishimoto, A. (2010). Alexithymia and its $R$ relationships with eating behavior, self esteem, and body esteem in college women. Kobe Journal of Medical Sciences (Vol. 56).

Scattolon, Y. S., \& Nicki, R. M. (1995). Worry as an Inhibitor of Dietary Restraint. Behavioural and Cognitive Psychotherapy, 23(1), 25-33. doi:10.1017/S1352465800017604

Schaefer, A., Nils, F., Sanchez, X., \& Philippot, P. (2010). Assessing the effectiveness of a large database of emotion-eliciting films: A new tool for emotion researchers. Cognition \& Emotion, 24(7), 1153-1172. doi:10.1080/02699930903274322

Schaefer, A., \& Philippot, P. (2005). Selective effects of emotion on the phenomenal characteristics of autobiographical memories. Memory, 13(2), 148-160. doi:10.1080/09658210344000648

Schaefer, L. M., \& Thompson, J. K. (2014). The development and validation of the Physical Appearance Comparison Scale-Revised (PACS-R). Eating Behaviors, 15(2), 209-217. doi:10.1016/j.eatbeh.2014.01.001 
Schandry, R. (1981). Heart beat perception and emotional experience. Psychophysiology, 18(4), 483-488. doi:10.1111/j.1469-8986.1981.tb02486.x

Schneider, K., Appelhans, B., \& Whited, M. (2010). Trait anxiety, but not trait anger, predisposes obese individuals to emotional eating. Appetite, 55(3), 701-706. doi:10.1016/j.appet.2010.10.006

Schulz, K. F., \& Grimes, D. A. (2002). Generation of allocation sequences in randomised trials: chance, not choice. The Lancet, 359(9305), 515-519. doi:10.1016/S0140-6736(02)07683-3

Schulz, S., \& Laessle, R. (2012). Stress-induced laboratory eating behavior in obese women with binge eating disorder. Appetite, 58(2), 457-461. doi:10.1016/J.APPET.2011.12.007

Schutz, H. K., \& Paxton, S. J. (2007). Friendship quality, body dissatisfaction, dieting and disordered eating in adolescent girls. British Journal of Clinical Psychology, 46(1), 67-83. doi:10.1348/014466506X115993

Sexton, M. C., Sunday, S. R., Hurt, S., \& Halmi, K. A. (1998). The relationship between alexithymia, depression, and axis II psychopathology in eating disorder inpatients. International Journal of Eating Disorders, 23(3), 277-286.

Shah, P., Hall, R., Catmur, C., \& Bird, G. (2016). Alexithymia, not autism, is associated with impaired interoception. Cortex, 81, 215-220. doi:10.1016/J.CORTEX.2016.03.021

Sheppard-Sawyer, C. L., McNally, R. J., \& Fischer, J. H. (2000). Film-induced sadness as a trigger for disinhibited eating. International Journal of Eating Disorders, 28(2), 215-220. doi:10.1002/1098-108X(200009)28:2<215::AIDEAT11>3.0.CO;2-J

Shishido, H., Gaher, R. R. M., \& Simons, J. J. S. (2013). I don't know how I feel, 
therefore I act: Alexithymia, urgency and alcohol problems. Addictive Behaviors, 38(4), 2014-2017. doi:10.1016/J.ADDBEH.2012.12.014

Sifneos, P. E. (1973). The prevalence of 'alexithymic' characteristics in psychosomatic patients. Psychotherapy and Psychosomatics, 22(2), 255-62. doi:10.1159/000286529

Sifneos, P. E. (1996). Alexithymia: Past and present. American Journal of Psychiatry. doi:10.1176/ajp.153.7.137

Smink, F. R. E., Van Hoeken, D., \& Hoek, H. W. (2012). Epidemiology of eating disorders: Incidence, prevalence and mortality rates. Current Psychiatry Reports, 14(4), 406-414. doi:10.1007/s11920-012-0282-y

Smyth, J. M., Wonderlich, S. A., Heron, K. E., Sliwinski, M. J., Crosby, R. D., Mitchell, J. E., \& Engel, S. G. (2007). Daily and momentary mood and stress are associated with binge eating and vomiting in bulimia nervosa patients in the natural environment. Journal of Consulting and Clinical Psychology, 75(4), 629638. doi:10.1037/0022-006X.75.4.629

Smyth, J. M., Wonderlich, S. A., Sliwinski, M. J., Crosby, R. D., Engel, S. G., Mitchell, J. E., \& Calogero, R. M. (2009). Ecological momentary assessment of affect, stress, and binge-purge behaviors: day of week and time of day effects in the natural environment. The International Journal of Eating Disorders, 42(5), 429-36. doi:10.1002/eat.20623

Smyth, J., Wonderlich, S., Crosby, R., Miltenberger, R., Mitchell, J., \& Rorty, M. (2001). The use of ecological momentary assessment approaches in eating disorder research. International Journal of Eating Disorders, 30(1), 83-95. doi:10.1002/eat.1057

Snoek, H. M., Engels, R. C. M. E., Janssens, J. M. A. M., \& van Strien, T. (2007). 
Parental behaviour and adolescents' emotional eating. Appetite, 49(1), 223-230. doi:10.1016/J.APPET.2007.02.004

Snoek, H. M., van Strien, T., Janssens, J. M. A. M., \& Engels, R. C. M. E. (2006). The effect of television viewing on adolescents' snacking: Individual differences explained by external, restrained and emotional eating. Journal of Adolescent Health, 39(3), 448-451. doi:10.1016/j.jadohealth.2005.12.020

Snoek, H. M., van Strien, T., Janssens, J. M. A. M., \& Engels, R. C. M. E. (2007). Emotional, external, restrained eating and overweight in Dutch adolescents. Scandinavian Journal of Psychology, 48(1), 23-32. doi:10.1111/j.14679450.2006.00568.x

Sozou, P. D., \& Seymour, R. M. (2003). Augmented discounting: Interaction between ageing and time-preference behaviour. Proceedings of the Royal Society B: Biological Sciences, 270(1519), 1047-1053. doi:10.1098/rspb.2003.2344

Spence, S., \& Courbasson, C. (2012). The role of emotional dysregulation in concurrent eating disorders and substance use disorders. Eating Behaviours, 13(4), 382-385. doi:10.1016/J.EATBEH.2012.05.006

Spencer, E. A., Appleby, P. N., Davey, G. K., \& Key, T. J. (2002). Validity of selfreported height and weight in 4808 EPIC-Oxford participants. Public Health Nutrition, 5(4), 561-565. doi:10.1079/PHN2001322

Speranza, M., Corcos, M., Loas, G., Stéphan, P., Guilbaud, O., Perez-Diaz, F., ... Flament, M. (2005). Depressive personality dimensions and alexithymia in eating disorders. Psychiatry Research, 135(2), 153-163.

Spoor, S. T. P., Bekker, M. H. J., van Strien, T., \& van Heck, G. L. (2007). Relations between negative affect, coping, and emotional eating. Appetite, 48(3), 368-376. doi:10.1016/J.APPET.2006.10.005 
Sproesser, G., Schupp, H., \& Renner, B. (2013). The bright side of stress-induced eating eating more when stressed but less when pleased. Psychological Science, 25(1), 58-65. doi:10.1177/0956797613494849

Stamatakis, E., Wardle, J., \& Cole, T. J. (2010). Childhood obesity and overweight prevalence trends in England: evidence for growing socioeconomic disparities. International Journal of Obesity, 34(1), 41-47. doi:10.1038/ijo.2009.217

Stanley, D. J., \& Meyer, J. P. (2009). Two-dimensional affective space: A new approach to orienting the axes. Emotion, 9(2), 214-237. Retrieved from http://psycnet.apa.org/doiLanding?doi=10.1037\%2Fa0014612

Stasiewicz, P. R., Bradizza, C. M., Gudleski, G. D., Coffey, S. F., Schlauch, R. C., Bailey, S. T., ... Gulliver, S. B. (2012). The relationship of alexithymia to emotional dysregulation within an alcohol dependent treatment sample. Addictive Behaviors, 37(4), 469-76. doi:10.1016/j.addbeh.2011.12.011

Stepp, S. D., Scott, L. N., Morse, J. Q., Nolf, K. A., Hallquist, M. N., \& Pilkonis, P. A. (2014). Emotion dysregulation as a maintenance factor of borderline personality disorder features. Comprehensive Psychiatry, 55(3), 657-666. doi:10.1016/j.comppsych.2013.11.006

Stevens, S., Gerlach, A. L., Cludius, B., Silkens, A., Craske, M. G., \& Hermann, C. (2011). Heartbeat perception in social anxiety before and during speech anticipation. Behaviour Research and Therapy, 49(2), 138-143. doi:10.1016/J.BRAT.2010.11.009

Stice, E. (1994). Review of the evidence for a sociocultural model of bulimia nervosa and an exploration of the mechanisms of action. Clinical Psychology Review, 14(7), 633-661. doi:10.1016/0272-7358(94)90002-7

Stice, E. (1998). Relations of restraint and negative affect to bulimic pathology: A 
longitudinal test of three competing models. International Journal of Eating Disorders, $\quad 23(3), \quad$ 243-260. doi:10.1002/(SICI)1098108X(199804)23:3<243::AID-EAT2>3.0.CO;2-J

Stice, E. (2001). A prospective test of the dual-pathway model of bulimic pathology: Mediating effects of dieting and negative affect. Journal of Abnormal Psychology, 110(1), 124-135. doi:10.1037/0021-843X.110.1.124

Stice, E., Fisher, M., \& Lowe, M. R. (2004). Are dietary restraint scales valid measures of acute dietary restriction? Unobtrusive observational data suggest not. Psychological Assessment, 16(1), 51-59. doi:10.1037/1040-3590.16.1.51

Stice E. (2002). Risk and maintenance factors for eating pathology: A meta -analytic review. Psychological Bulletin, 128(128), 825-848. Retrieved from http://psycnet.apa.org/buy/2002-15487-007

Striegel-Moore, R., McAvay, G., \& Rodin, J. (1986). Psychological and behavioral correlates of feeling fat in women. International Journal of Eating Disorders, 5(5), 935-947. doi:10.1002/1098-108X(198607)5:5<935::AIDEAT2260050514>3.0.CO;2-0

Strother, E., Lemberg, R., Stanford, S. C., \& Turberville, D. (2012). Eating Disorders in Men: Underdiagnosed, Undertreated, and Misunderstood. Eating Disorders, 20(5), 346-355. doi:10.1080/10640266.2012.715512

Stunkard, A. J., \& Messick, S. (1985). The three-factor eating questionnaire to measure dietary restraint, disinhibition and hunger, 29(1), 71-83. doi:10.1016/00223999(85)90010-8

Suengas, A. G., \& Johnson, M. K. (1988). Qualitative effects of rehearsal on memories for perceived and imagined complex events. Journal of Experimental Psychology: General, 117(4), 377-389. doi:10.1037/0096-3445.117.4.377 
Sung, J., Lee, K., \& Song, Y.-M. (2009). Relationship of eating behavior to long-term weight change and body mass index: The healthy twin study. Eating and Weight Disorders - Studies on Anorexia, Bulimia and Obesity, 14(2-3), e98-e105. doi:10.1007/BF03327806

Suslow, T., \& Donges, U. S. (2017). Alexithymia components are differentially related to explicit negative affect but not associated with explicit positive affect or implicit affectivity. Frontiers in Psychology, 8, 1758. doi:10.3389/fpsyg.2017.01758

Suter, P. M., \& Tremblay, A. (2005). Is alcohol consumption a risk factor for weight gain and obesity? Critical Reviews in Clinical Laboratory Sciences, 42(3), 197227. doi:10.1080/10408360590913542

Swanson, S. A., Crow, S. J., Grange, D. Le, Swendsen, J., \& Merikangas, K. R. (2011). Prevalence and Correlates of Eating Disorders in Adolescents. Archives of General Psychiatry, 68(7), 714. doi:10.1001/archgenpsychiatry.2011.22

Swart, M., Kortekaas, R., \& Aleman, A. (2009). Dealing with feelings: Characterization of trait Alexithymia on emotion regulation strategies and cognitive-emotional processing. PLoS ONE, 4(6), e5751. doi:10.1371/journal.pone.0005751

Tanofsky, M. B., Wilfley, D. E., Spurrell, E. B., Welch, R., \& Brownell, K. D. (1997). Comparison of men and women with binge eating disorder. International Journal of Eating Disorders, 21(1), 49-54. doi:10.1002/(SICI)1098108X(199701)21:1<49::AID-EAT6>3.0.CO;2-3

Tapper, K., Shaw, C., Ilsley, J., Hill, A. J., Bond, F. W., \& Moore, L. (2009). Exploratory randomised controlled trial of a mindfulness-based weight loss intervention for women. Appetite, 52(2), 396-404. 
doi:10.1016/j.appet.2008.11.012

Taylor, G. (2000). Recent Developments in Alexithymia Theory and Research. Canadian Journal of Psychiatry, 45(March), 134-142. doi:10.1177/070674370004500203

Taylor, G., Bagby, R., \& Parker, J. (2003). The 20-Item Toronto Alexithymia Scale: IV. Reliability and factorial validity in different languages and cultures. Journal of Psychosomatic Research, 55(3), 277-283.

Taylor, G. J., Bagby, M. R., \& Parker, J. D. A. (1992). Toronto Alexithymia Scale Manual.

Taylor, G. J., \& Bagby, R. M. (1988). Measurement of alexithymia. Recommendations for clinical practice and future research. The Psychiatric Clinics of North America, 11(3), 351-66. doi:10.1016/S0193-953X(18)30486-6

Taylor, G. J., Bagby, R. M., \& Parker, J. D. A. (1997). Disorders of Affect Regulation: Alexithymia in Medical and Psychiatric Illness. New York, USA: Cambridge University Press. doi:10.1017/CBO9780511526831

Taylor, G. J., Bagby, R. M., \& Parker, J. D. A. (2006). The 20-item Toronto $\begin{array}{llll}\text { Alexithymia } & \text { Scale } & \text { Manual. } & \text { Retrieved }\end{array}$ http://www.gtaylorpsychiatry.org/tas.htm.

Taylor, G. J., Michael Bagby, R., \& Parker, J. D. A. (1991). The alexithymia construct: a potential paradigm for psychosomatic medicine. Psychosomatics, 32(2), 153164.

Taylor, G. J., Parker, J. G. A., Bagby, R. M., \& Bourke, M. P. (1996). Relationships between alexithymia and psychological characteristics associated with eating disorders. Journal of Psychosomatic Research, 41(6), 561-568.

Taylor, G. J., Ryan, D., \& Bagby, R. M. (1985). Toward the development of a new 
self-report alexithymia scale. Psychotherapy and Psychosomatics, 44(4), 191-9. doi:10.1159/000287912

Telch, C. F., Agras, W. S., \& Linehan, M. M. (2000). Group dialectical behavior therapy for binge-eating disorder: A preliminary, uncontrolled trial. Behavior Therapy, 31(3), 569-582. doi:10.1016/S0005-7894(00)80031-3

Telch, C. F., Agras, W. S., \& Linehan, M. M. (2001). Dialectical behavior therapy for binge eating disorder. Journal of Consulting and Clinical Psychology, 69(6), 1061-1065. doi:10.1037/0022-006X.69.6.1061

Teng, H.-C., Yeh, M.-L., \& Wang, M.-H. (2018). Walking with controlled breathing improves exercise tolerance, anxiety, and quality of life in heart failure patients: A randomized controlled trial. European Journal of Cardiovascular Nursing, 1(11). doi:10.1177/1474515118778453

Thayer, R. E. (2001). Calm energy: How people regulate mood with food and exercise. New York, USA: Oxford University Press, USA.

Tice, D. M., Bratslavsky, E., \& Baumeister, R. F. (2001). Emotional distress regulation takes precedence over impulse control: If you feel bad, do it! Journal of Personality and Social Psychology, 80(1), 53-67. doi:10.1037/00223514.80 .1 .53

Tiggemann, M. (1996). 'Thinking' versus 'feeling' fat: Correlates of two indices of body image dissatisfaction. Australian Journal of Psychology, 48(1), 21-25. doi:10.1080/00049539608259501

Timmerman, G. M., \& Acton, G. J. (2001). The relationship between basic need satisfaction and emotional eating. Issues in Mental Health Nursing, 22(7), 691701. doi:10.1080/016128401750434482

Todarello, O., Taylor, G. J., Parker, J. D. A., \& Fanelli, M. (1995). Alexithymia in 
essential hypertensive and psychiatric outpatients: A comparative study. Journal of Psychosomatic Research, 39(8), 987-994. doi:10.1016/0022-3999(95)005064

Tolbaños, L., Miró, M. T., Ibáñez, I., \& Betancort, M. (2017). YOGA and selfregulation in management of essential arterial hypertension and associated emotional symptomatology: A randomized controlled trial. Complementary Therapies in Clinical Practice, 29, 153-161. doi:10.1016/j.ctcp.2017.09.012

Toner, B. B., Garfinkel, P. E., \& Garner, D. M. (1987). Cognitive style of patients with bulimic and diet-restricting anorexia nervosa. American Journal of Psychiatry, 144(4), 510-512. doi:10.1176/ajp.144.4.510

Torres, S., Guerra, M. P., Lencastre, L., Miller, K., Vieira, F. M., Roma-Torres, A., ... Costa, P. (2015). Alexithymia in anorexia nervosa: The mediating role of depression. Psychiatry Research, 225(1), 99-107. doi:10.1016/j.psychres.2014.10.023

Torres, S. J., \& Nowson, C. A. (2007). Relationship between stress, eating behavior, and obesity. Nutrition, 23(11-12), 887-894. doi:10.1016/j.nut.2007.08.008

Turner, S., Luszczynska, A., Warner, L., \& Schwarzer, R. (2010). Emotional and uncontrolled eating styles and chocolate chip cookie consumption. A controlled trial of the effects of positive mood enhancement. Appetite, 54, 143-149. doi:10.1016/j.appet.2009.09.020

Udo, T., Grilo, C. M., Brownell, K. D., Weinberger, A. H., DiLeone, R. J., \& McKee, S. A. (2013). Modeling the effects of positive and negative mood on the ability to resist eating in obese and non-obese individuals. Eating Behaviors, 14(1), 4046. doi:10.1016/J.EATBEH.2012.10.010

Van de Velde, S., Bracke, P., \& Levecque, K. (2010). Gender differences in depression 
in 23 European countries. Cross-national variation in the gender gap in depression. Social Science \& Medicine, 71(2), 305-313. doi:10.1016/J.SOCSCIMED.2010.03.035

van Strien, T. (2000). Ice-cream consumption, tendency toward overeating, and personality. International Journal of Eating Disorders, 28(4), 460-464. doi:10.1002/1098-108X(200012)28:4<460::AID-EAT16>3.0.CO;2-A

van Strien, T., \& Bazelier, F. G. (2007). Perceived parental control of food intake is related to external, restrained and emotional eating in 7-12-year-old boys and girls. Appetite, 49(3), 618-625. doi:10.1016/J.APPET.2007.03.227

van Strien, T., Cebolla, A., Etchemendy, E., Gutiérrez-Maldonado, J., Ferrer-García, M., Botella, C., \& Baños, R. (2013). Emotional eating and food intake after sadness and joy. Appetite, 66, 20-25. doi:10.1016/j.appet.2013.02.016

van Strien, T., Engels, R., van Leeuwe, J., \& Snoek, H. (2005). The Stice model of overeating: tests in clinical and non-clinical samples. Appetite, 45, 205-213.

van Strien, T., Frijters, J. E. R., Bergers, G. P. A., \& Defares, P. B. (1986). The Dutch Eating Behavior Questionnaire (DEBQ) for assessment of restrained, emotional, and external eating behavior. International Journal of Eating Disorders, 5(2), $295-315$. doi:10.1002/1098-108X(198602)5:2<295::AIDEAT2260050209>3.0.CO;2-T

van Strien, T., Herman, C. P., Anschutz, D. J., Engels, R. C. M. E., \& de Weerth, C. (2012). Moderation of distress-induced eating by emotional eating scores. Appetite, 58(1), 277-284. doi:10.1016/J.APPET.2011.10.005

van Strien, T., Herman, C. P., \& Verheijden, M. W. (2009). Eating style, overeating, and overweight in a representative Dutch sample. Does external eating play a role? Appetite, 52(2), 380-387. doi:10.1016/j.appet.2008.11.010 
Van Strien, T., Konttinen, H., Homberg, J. R., Engels, R. C. M. E., \& Winkens, L. H. H. (2016). Emotional eating as a mediator between depression and weight gain. Appetite, 100, 216-224. doi:10.1016/j.appet.2016.02.034

van Strien, T., \& Ouwens, M. A. (2007). Effects of distress, alexithymia and impulsivity on eating. Eating Behaviors, 8(2), 251-257. doi:10.1016/J.EATBEH.2006.06.004

van Strien, T., Ouwens, M. A., Engel, C., \& de Weerth, C. (2014). Hunger, inhibitory control and distress-induced emotional eating. Appetite, 79, 124-133. doi:10.1016/j.appet.2014.04.020

van Strien, T., Peter Herman, C., \& Verheijden, M. W. (2012). Eating style, overeating and weight gain. A prospective 2-year follow-up study in a representative Dutch sample. Appetite, 59(3), 782-789. doi:10.1016/J.APPET.2012.08.009

van Strien, T., van de Laar, F. A., van Leeuwe, J. F. J., Lucassen, P. L. B. J., van den Hoogen, H. J. M., Rutten, G. E. H. M., \& van Weel, C. (2007). The dieting dilemma in patients with newly diagnosed type 2 diabetes: Does dietary restraint predict weight gain 4 years after diagnosis? Health Psychology, 26(1), 105-112. doi:10.1037/0278-6133.26.1.105

Västfjäll, D. (2001). Emotion induction through music: A review of the musical mood induction procedure. Musicae Scientiae, 5, 173-211. doi:10.1177/10298649020050S107

Velten, E. (1968). A laboratory task for induction of mood states. Behaviour Research and Therapy, 6(4), 473-482. doi:10.1016/0005-7967(68)90028-4

Vorst, H. C. ., \& Bermond, B. (2001). Validity and reliability of the Bermond-Vorst Alexithymia Questionnaire. Personality and Individual Differences, 30(3), 413434. doi:10.1016/S0191-8869(00)00033-7 
Waller, G., \& Osman, S. (1998). Emotional eating and eating psychopathology among non-eating-disordered women. International Journal of Eating Disorders, 23(4), 419-424. doi:10.1002/(SICI)1098-108X(199805)23:4<419::AIDEAT9>3.0.CO;2-L

Wallis, D. J., \& Hetherington, M. M. (2004). Stress and eating: the effects of egothreat and cognitive demand on food intake in restrained and emotional eaters. Appetite, 43(1), 39-46. doi:10.1016/J.APPET.2004.02.001

Wallis, D. J., \& Hetherington, M. M. (2009). Emotions and eating. Self-reported and experimentally induced changes in food intake under stress. Appetite, 52(2), 355362. doi:10.1016/J.APPET.2008.11.007

Wannamethee, S. G., \& Shaper, A. G. (2003). Alcohol, body weight, and weight gain in middle-aged men. The American Journal of Clinical Nutrition, 77(5), 13121317. doi:10.1093/ajen/77.5.1312

Wardle, J. (1987). Eating style: a validation study of the Dutch Eating Behaviour Questionnaire in normal subjects and women with eating disorders. Journal of Psychosomatic Research, 31(2), 161-9. doi:10.1016/0022-3999(87)90072-9

Wardle, J., Marsland, L., Sheikh, Y., Quinn, M., Fedoroff, I., \& Ogden, J. (1992). Eating style and eating behaviour in adolescents. Appetite, 18(3), 167-183. doi:10.1016/0195-6663(92)90195-C

Wardle, J., Sanderson, S., Guthrie, C. A., Rapoport, L., \& Plomin, R. (2002). Parental Feeding Style and the Inter-generational Transmission of Obesity Risk. Obesity Research, 10(6), 453-462. doi:10.1038/oby.2002.63

Wardle, J., Steptoe, A., Oliver, G., \& Lipsey, Z. (2000). Stress, dietary restraint and food intake. Journal of Psychosomatic Research, 48(2), 195-202. doi:10.1016/S0022-3999(00)00076-3 
Watson, D., \& Clark, L. A. (1999). The PANAS-X: Manual for the Positive and Negative Affect Schedule - Expanded Form. Iowa Research Online, 277(6), 127. doi:10.1111/j.1742-4658.2010.07754.X

Watson, D., Clark, L. A., \& Tellegen, A. (1988). Development and validation of brief measures of positive and negative affect: The PANAS scales. Journal of Personality and Social Psychology, 54(6), 1063-1070.

Waxman, S. E. (2009). A Systematic Review of Impulsivity in Eating Disorders. Eating Disorders, 17, 408-425. doi:10.1002/erv.952

Wegner, K. E., Smyth, J. M., Crosby, R. D., Wittrock, D., Wonderlich, S. A., \& Mitchell, J. E. (2002). An evaluation of the relationship between mood and binge eating in the natural environment using ecological momentary assessment. International Journal of Eating Disorders, 32(3), 352-361. doi:10.1002/eat.10086

Weller, R. E., Cook, E. W., Avsar, K. B., \& Cox, J. E. (2008). Obese women show greater delay discounting than healthy-weight women. Appetite, 51(3), 563-569. doi:10.1016/j.appet.2008.04.010

Werthmann, J., Renner, F., Roefs, A., Huibers, M. J. H., Plumanns, L., Krott, N., \& Jansen, A. (2014). Looking at food in sad mood: Do attention biases lead emotional eaters into overeating after a negative mood induction? Eating Behaviors, 15(2), 230-236. doi:10.1016/j.eatbeh.2014.02.001

Westerterp-Plantenga, M. S. (2004). Effects of energy density of daily food intake on long-term energy intake. Physiology \& Behavior, 81(5), 765-771. doi:10.1016/J.PHYSBEH.2004.04.030

Wheeler, K., Greiner, P., \& Boulton, M. (2005). Exploring alexithymia, depression, and binge eating in self-reported eating disorders in women. Perspectives in 
Psychiatric Care, 41(3), 114-123.

Whiteside, S. P., \& Lynam, D. R. (2001). The Five Factor Model and impulsivity: using a structural model of personality to understand impulsivity. Personality and Individual Differences, 30(4), 669-689. doi:10.1016/S0191-8869(00)00064-7

WHO. (2017). WHO | Facts and figures on childhood obesity. WHO. Retrieved from http://www.who.int/end-childhood-obesity/facts/en/

WHO. (2018). Body mass index - BMI. Retrieved from http://www.euro.who.int/en/health-topics/disease-prevention/nutrition/ahealthy-lifestyle/body-mass-index-bmi

Wilding, J. P. H. (2001). Causes of obesity. Practical Diabetes International, 18(8), 288-292. doi:10.1002/pdi.277

Wilkinson, L. L., Rowe, A. C., Bishop, R. J., \& Brunstrom, J. M. (2010). Attachment anxiety, disinhibited eating, and body mass index in adulthood. International Journal of Obesity, 34(9), 1442-1445. doi:10.1038/ijo.2010.72

Wilkinson, L. L., Rowe, A. C., \& Heath, G. H. (2013). Eating me up inside: Priming attachment security and anxiety, and their effects on snacking. Journal of Social and Personal Relationships, 30(6), 795-804. doi:10.1177/0265407512468371

Wilkinson, L. L., Rowe, A. C., Robinson, E., \& Hardman, C. A. (2018). Explaining the relationship between attachment anxiety, eating behaviour and BMI. Appetite, 127, 214-222. doi:10.1016/J.APPET.2018.04.029

Wilkinson, N., \& Klaes, M. (2012). An Introduction to Behavioral Economics. New York, USA: Palgrave Macmillan.

Williams, T., \& Kelley, C. (2015). Gnuplot. Retrieved from http://www.gnuplot.info/docs_5.0/gnuplot.pdf

Wise, T. N., Mann, L. S., Mitchell, J. D., Hryvniak, M., \& Hill, B. (1990). Secondary 
alexithymia: An empirical validation. Comprehensive Psychiatry, 31(4), 284288. doi:10.1016/0010-440X(90)90035-Q

Wiser, S., \& Telch, C. F. (1999). Dialectical behavior therapy for binge-eating disorder. Journal of Clinical Psychology, 55(6), 755-768. doi:10.1002/(SICI)1097-4679(199906)55:6<755::AID-JCLP8>3.0.CO;2-R

Wood, R. L., \& McHugh, L. (2013). Decision making after traumatic brain injury: A temporal discounting paradigm. Journal of the International Neuropsychological Society, 19(2), 181-188. doi:10.1017/S135561771200118X

Wood, R. L., O’Hagan, G., Williams, C., McCabe, M., \& Chadwick, N. (2014). Anxiety Sensitivity and Alexithymia as Mediators of Postconcussion Syndrome Following Mild Traumatic Brain Injury. Journal of Head Trauma Rehabilitation, 29(1), E9-E17. doi:10.1097/HTR.0b013e31827eabba

Wood, R. L., \& Williams, C. (2007). Neuropsychological correlates of organic alexithymia. Journal of the International Neuropsychological Society, 13(3), 471-479. doi:10.1017/S1355617707070518

Wood, R. L., Williams, C., \& Lewis, R. (2010). Role of alexithymia in suicide ideation after traumatic brain injury. Journal of the International Neuropsychological Society, 16(06), 1108-1114. doi:10.1017/S1355617710001013

Wood, W., Rhodes, N., \& Whelan, M. (1989). Sex differences in positive well-being: A consideration of emotional style and marital status. Psychological Bulletin, 106(2), 249-264. doi:10.1037/0033-2909.106.2.249

Wright, S. M., \& Aronne, L. J. (2012). Causes of obesity. Abdominal Imaging, 37(5), 730-732. doi:10.1007/s00261-012-9862-x

Yeomans, M. R., \& Coughlan, E. (2009). Mood-induced eating. Interactive effects of restraint and tendency to overeat. Appetite, 52(2), 290-298. 
doi:10.1016/J.APPET.2008.10.006

Yeomans, M. R., Leitch, M., \& Mobini, S. (2008). Impulsivity is associated with the disinhibition but not restraint factor from the Three Factor Eating Questionnaire. Appetite, 50(2-3), 469-476. doi:10.1016/j.appet.2007.10.002

Yi, R., Landes, R. D., \& Bickel, W. K. (2009). Novel models of intertemporal valuation: Past and future outcomes. Journal of Neuroscience, Psychology, and Economics, 2(2), 102-111. doi:10.1037/a0017571

Young, H. A., Williams, C., Pink, A. E., Freegard, G., Owens, A., \& Benton, D. (2017). Getting to the heart of the matter: Does aberrant interoceptive processing contribute towards emotional eating? PLOS ONE, 12(10), e0186312. doi:10.1371/journal.pone.0186312

Zamariola, G., Vlemincx, E., Luminet, O., \& Corneille, O. (2018). Relationship between interoceptive accuracy, interoceptive sensibility, and alexithymia. Personality and Individual Differences, 125, 14-20. doi:10.1016/j.paid.2017.12.024

Zech, E., Luminet, O., Rimé, B., \& Wagner, H. (1999). Alexithymia and its measurement: confirmatory factor analyses of the 20-item Toronto Alexithymia Scale and the Bermond-Vorst Alexithymia Questionnaire. European Journal of Personality, $\quad$ 13(6), 511-532. doi:10.1002/(SICI)10990984(199911/12)13:6<511::AID-PER347>3.0.CO;2-0

Zeeck, A., Stelzer, N., Linster, H. W., Joos, A., \& Hartmann, A. (2011). Emotion and eating in binge eating disorder and obesity. European Eating Disorders Review, $19(5), 426-437$.

Zhao, G., Ford, E. S., Dhingra, S., Li, C., Strine, T. W., \& Mokdad, A. H. (2009). Depression and anxiety among US adults: Associations with body mass index. 
International Journal of Obesity. doi:10.1038/ijo.2008.268

Zigmond, A. S., \& Snaith, R. P. (1983). The Hospital Anxiety and Depression Scale. Acta Psychiatrica Scandinavica, 67(6), 361-370. doi:10.1111/j.16000447.1983.tb09716.x

Zimmermann, G., Rossier, J., De Stadelhofen, F. M., \& Gaillard, F. (2005). Alexithymia assessment and relations with dimensions of personality. European Journal of Psychological Assessment, 21(1), 23-33. doi:10.1027/10155759.21 .1 .23

Zlotnick, C., Shea, M. T., Pearlstein, T., Simpson, E., Costello, E., \& Begin, A. (1996). The relationship between dissociative symptoms, alexithymia, impulsivity, sexual abuse, and self-mutilation. Comprehensive Psychiatry, 37(1), 12-16. doi:10.1016/S0010-440X(96)90044-9 
Appendices 
Appendix A. Study One: Ethics Memo

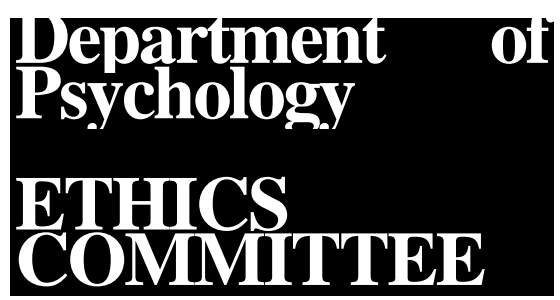

\title{
Memo
}

\author{
To: \\ Aimee Pink \\ From: Dr. Jeremy Tree \\ for Departmental Ethics Committee \\ Copy: $\quad$ Dr. Claire Williams \\ Date: $\quad 1^{\text {th }}$ November, 2014 \\ Re: An investigation into the relationship between \\ alexithymia, impulsivity, emotional eating and \\ delayed discounting
}

Your proposed study "An investigation into the relationship between alexithymia, impulsivity, emotional eating and delayed discounting", has been reviewed and is approved. Provided that the information obtained is kept absolutely confidential and that no personally identifiable information is entered on computer, you may proceed with your studies.

Please ensure that the signed copy of this Ethical Approval, together with any other paperwork associated with your research, is included in your final write up.

In order for your study to be displayed on the Experiment Management System (Participant Pool):

Forward this approval via email to Dr. Irene Reppa

\section{$\underline{\text { AND }}$}

Send a request for your study to be made visible, via the link on the EMS website (see Researcher Documentation for details). 


\section{Appendix B. Study One: Demographics}

Please complete the following questions on demographic information.

* 1 . What is your gender?

Female

Male

* 2. What is your date of birth Format: dd/mm/yyyy

-

*3. What is your marital status?

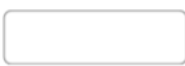

4. What is your ethnic group?

Choose one option that best describes your ethnic group or background.

Asian/Asian British (Indian, Pakistani, Bangladeshi, other Asian)

Black/ Black British (Black Caribbean, African, other Black)

Chinese

Mixed Ethnic Group (White and Black Caribbean, White and Black African, White and Asian, other Mixed)

White (White British/ White Irish/other)

Prefer not to say

Other Ethnic Group

Other (please specify)

* 5 . What is your current employment status?

6. What is your current occupation/ job title?

7. What is your current yearly income? (please specify to the nearest $£ 1000$ )

\section{(please specify to the nearest e1000)}

* 8. What is your highest qualification to date?

E.g. GCSE, BSc. 
* 9. Have you ever been diagnosed with an eating disorder?

Yes

No

If yes, please specify

* 10. Do you currently smoke cigarettes, or not?

Yes, I do

No, I do not

If yes, about how many cigarettes do you smoke in a typical day?

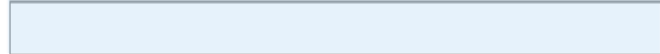

* 11. On average, how many units of alcohol do you consume a week?

E.g. A $25 \mathrm{ml}$ serving of spirits is 1 unit, a standard $175 \mathrm{ml}$ glass of wine is 2 units

* 12. Have you ever been diagnosed with a substance use disorder or addictive disorder? E.g. alcohol, drugs, gambling

Yes

No

If yes, please specify 


\section{Appendix C. The Positive Affect and Negative Affect Scale}

Watson, Clark, \& Tellegen (1988)

This scale consists of a number of words that describe different feelings and emotions. Read each item and then check the appropriate answer next to that word. Indicate to what extent you may have felt this way during the PAST WEEK.

* 13. Positive Affect and Negative Affect Scale

$$
\text { Very slightly or not }
$$

$$
\text { at all }
$$

A little

Moderately

Quite a bit

Extremely

Interested

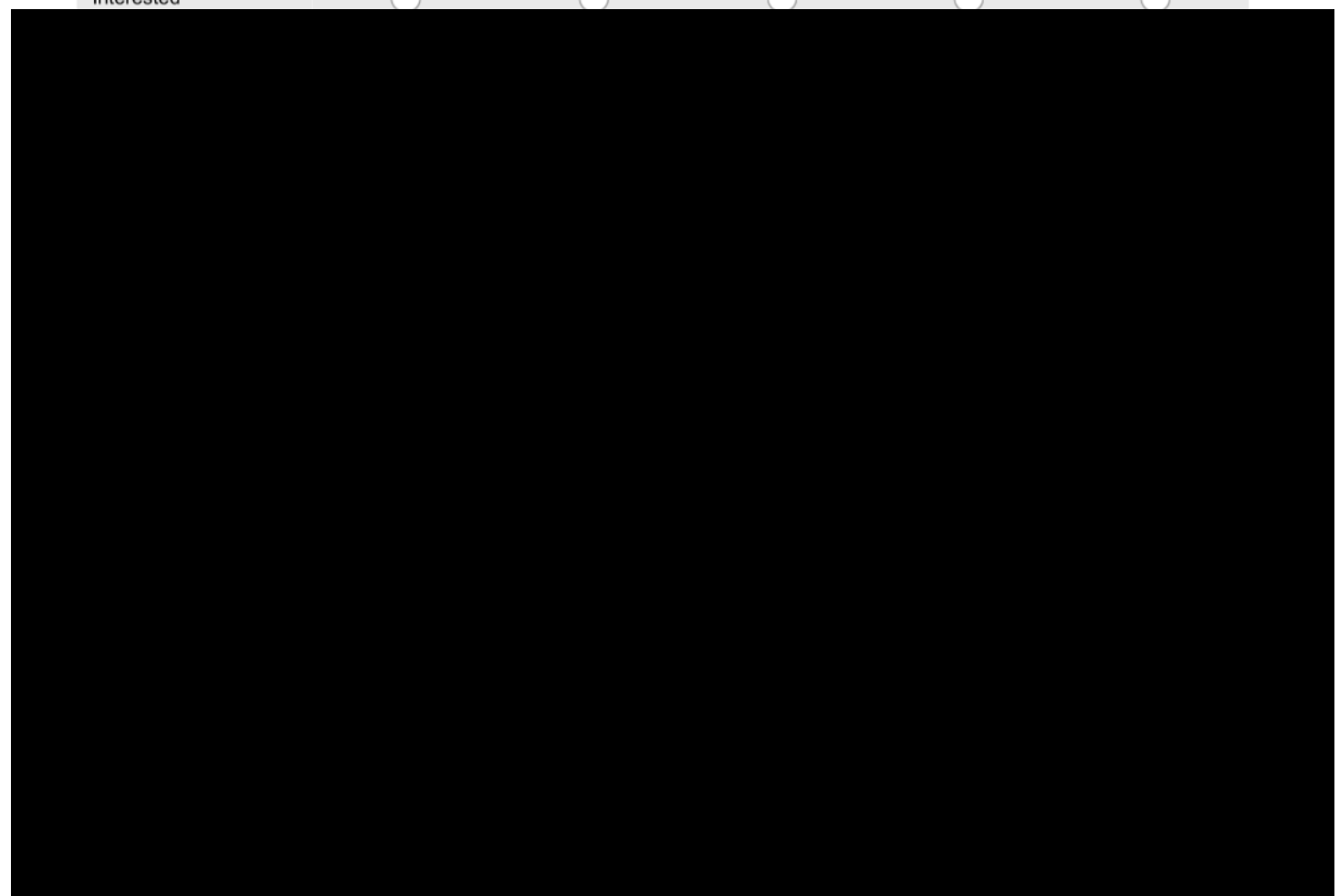

Active

Afraid 


\section{Appendix D. Toronto Alexithymia Scale}

Bagby, Parker, \& Taylor (1994)

Using the scale provided as a guide, indicate how much you agree or disagree with each of the

following statements by checking the corresponding circle. Give only one answer for each

statement.

* 14. Alexithymia

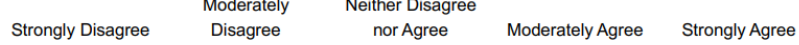

I am often confused

about what emotion I am

feeling.

It is difficult for me to find

the right words for my 


\section{Appendix E. UPPS-P Impulsivity Scale}

Cyders et al. (2007)

Below are a number of statements that describe ways in which people act and think. For each statement, please indicate how much you agree or disagree with the statement. If you Agree Strongly circle 1, if you Agree Somewhat circle 2, if you Disagree somewhat circle 3, and if you Disagree Strongly circle 4. Be sure to indicate your agreement or disagreement for every statement below. Also, there are questions on the following pages.

1. I have a reserved and cautious attitude toward life.

2. I have trouble controlling my impulses.

3. I generally seek new and exciting experiences and sensations.

4. I generally like to see things through to the end.

5. When I am very happy, I can't seem to stop myself from doing things that can have bad consequences.

6. My thinking is usually careful and purposeful.

7. I have trouble resisting my cravings (for food, cigarettes, etc.).

8. I'll try anything once.

9. I tend to give up easily.

10. When I am in great mood, I tend to get into situations that could cause me problems.

11. I am not one of those people who blurt out things without thinking.

12. I often get involved in things I later wish I could get out of.

13. I like sports and games in which you have to choose your next move very quickly.

14. Unfinished tasks really bother me.

15. When I am very happy, I tend to do things that may cause problems in my life.

16. I like to stop and think things over before I do them.

17. When I feel bad, I will often do things I later regret in order to make myself feel better now.

18. I would enjoy water skiing.

19. Once I get going on something I hate to stop.

20. I tend to lose control when I am in a great mood.

21. I don't like to start a project until I know exactly how to proceed. 
22. Sometimes when I feel bad, I can't seem to stop what I am doing even though it is making me feel worse.

23. I quite enjoy taking risks.

24. I concentrate easily.

25 . When I am really ecstatic, I tend to get out of control.

26. I would enjoy parachute jumping.

27. I finish what I start.

28. I tend to value and follow a rational, "sensible" approach to things.

29. When I am upset I often act without thinking.

30. Others would say I make bad choices when I am extremely happy about something.

31. I welcome new and exciting experiences and sensations, even if they are a little frightening and unconventional.

32. I am able to pace myself so as to get things done on time.

33. I usually make up my mind through careful reasoning.

34. When I feel rejected, I will often say things that I later regret.

35. Others are shocked or worried about the things I do when I am feeling very excited.

36. I would like to learn to fly an airplane.

37. I am a person who always gets the job done.

38. I am a cautious person.

39. It is hard for me to resist acting on my feelings.

40. When I get really happy about something, I tend to do things that can have bad consequences.

41. I sometimes like doing things that are a bit frightening.

42. I almost always finish projects that I start.

43. Before I get into a new situation I like to find out what to expect from it.

44. I often make matters worse because I act without thinking when I am upset.

45. When overjoyed, I feel like I can't stop myself from going overboard.

46. I would enjoy the sensation of skiing very fast down a high mountain slope.

47. Sometimes there are so many little things to be done that I just ignore them all.

48. I usually think carefully before doing anything.

49. When I am really excited, I tend not to think of the consequences of my actions.

50. In the heat of an argument, I will often say things that I later regret.

51. I would like to go scuba diving.

52. I tend to act without thinking when I am really excited.

53. I always keep my feelings under control. 
54. When I am really happy, I often find myself in situations that I normally wouldn't be comfortable with.

55. Before making up my mind, I consider all the advantages and disadvantages.

56. I would enjoy fast driving.

57. When I am very happy, I feel like it is ok to give in to cravings or overindulge.

58. Sometimes I do impulsive things that I later regret.

59. I am surprised at the things I do while in a great mood. 


\section{Appendix F. Beck Depression Inventory II}

Beck, Steer, \& Brown (1988)

The questionnaire consists of $\mathbf{2 1}$ groups of statements. Please read each group of statements carefully, and then pick out the one statement in each group that best describes the way you have been feeling during the PAST TWO WEEKS, INCLUDING TODAY. Check the circle beside the statement you have picked. If several statements in the group seem to apply equally well, circle the highest number in that group. Be sure that you do no choose more than one statement for any group, including item 16 (Changes in Sleep Pattern) or Item 18 (Changes in Appetite).

16. Sadness

I do not feel sad

I feel sad much of the time.

I am sad all of the time

tam so sad or unhappy that 1 can't stand t.

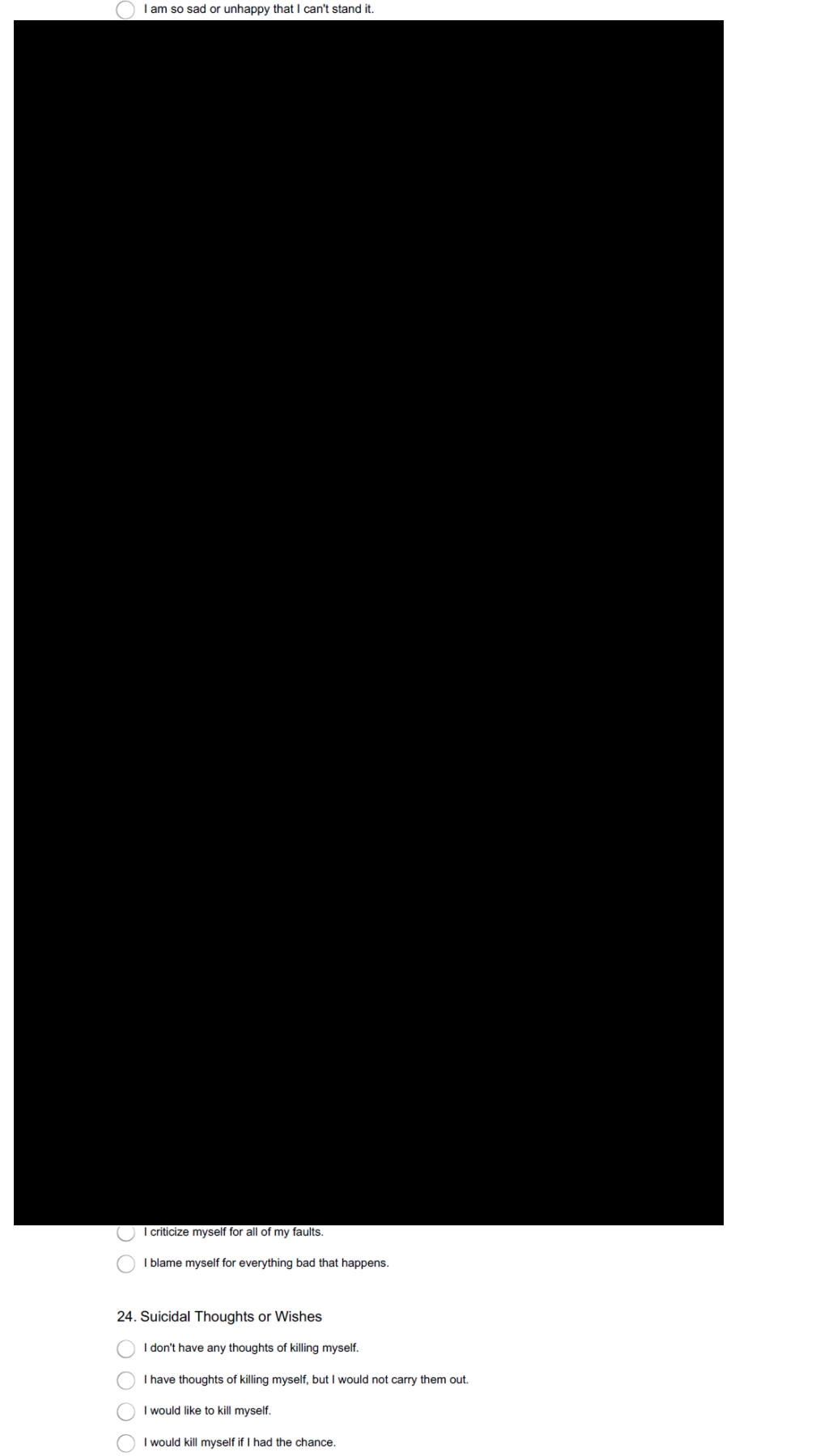




\section{Crying}

I don't cry anymore than I used to.

I cry more than I used to.

I cry more than I used to.

I feel like crying, but I can't.

\section{Agitation}

I am no more restless or wound up than usual.

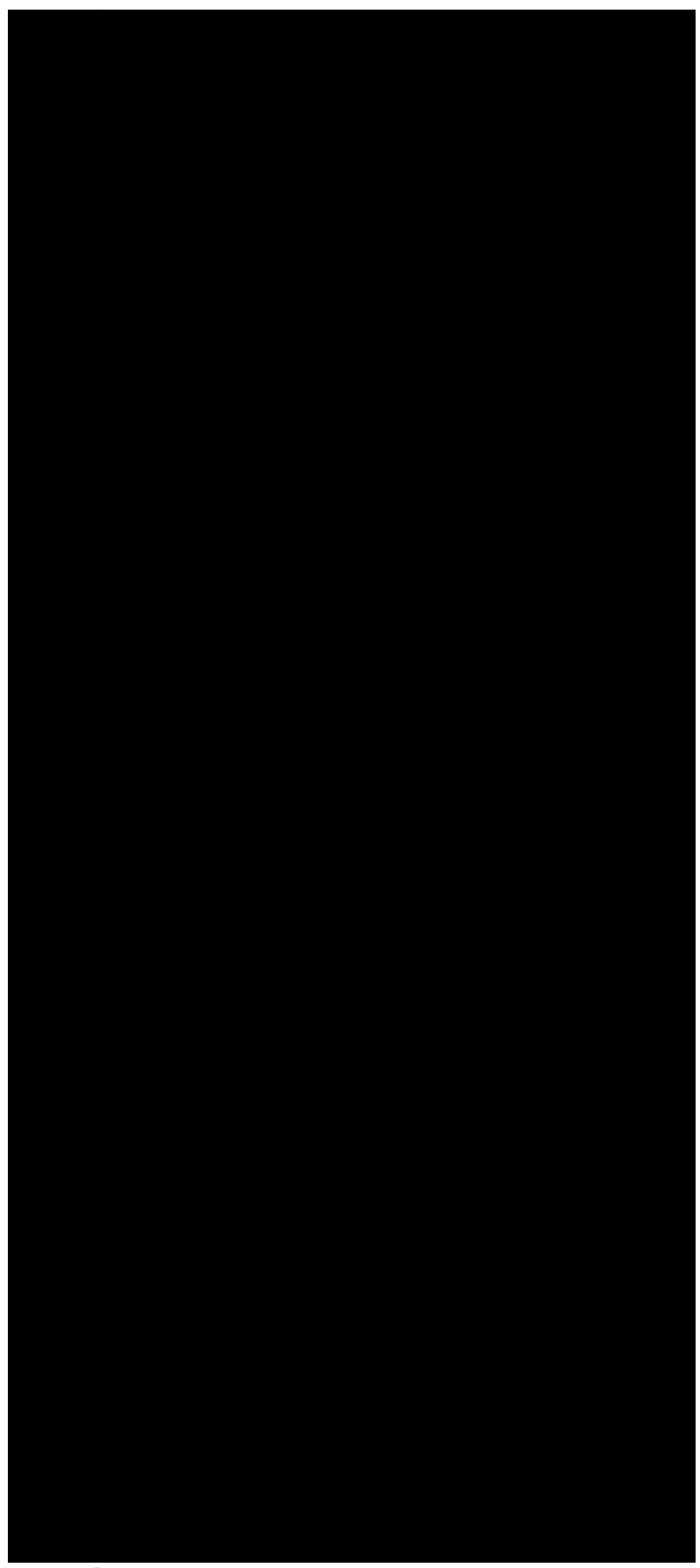

I have no appetite at all/ I crave food all the time.

34. Concentration Difficulty

I can concentrate as well as ever.

I can't concentrate as well as usual.

It's hard to keep my mind on anything for very long

I find I can't concentrate on anything. 
35. Tiredness or Fatigue

I am no more tired or fatigued than usual.

I get more tired or fatigued more easily than usual.

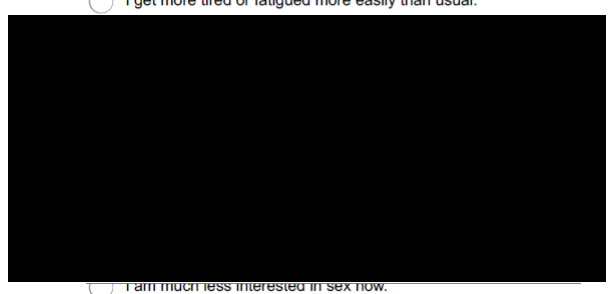

I have lost interest in sex completely.

388 | P a g e 


\section{Appendix G. Beck Anxiety Inventory}

\section{Beck, Epstein, Brown, Steer (1988)}

Below is a short list of common symptoms of anxiety. Please carefully read each item in the list. Indicate how much you have been bothered by each symptom during the PAST WEEK, INCLUDING TODAY, by checking the box in the corresponding space in the column next to each symptom.

* 37. Anxiety

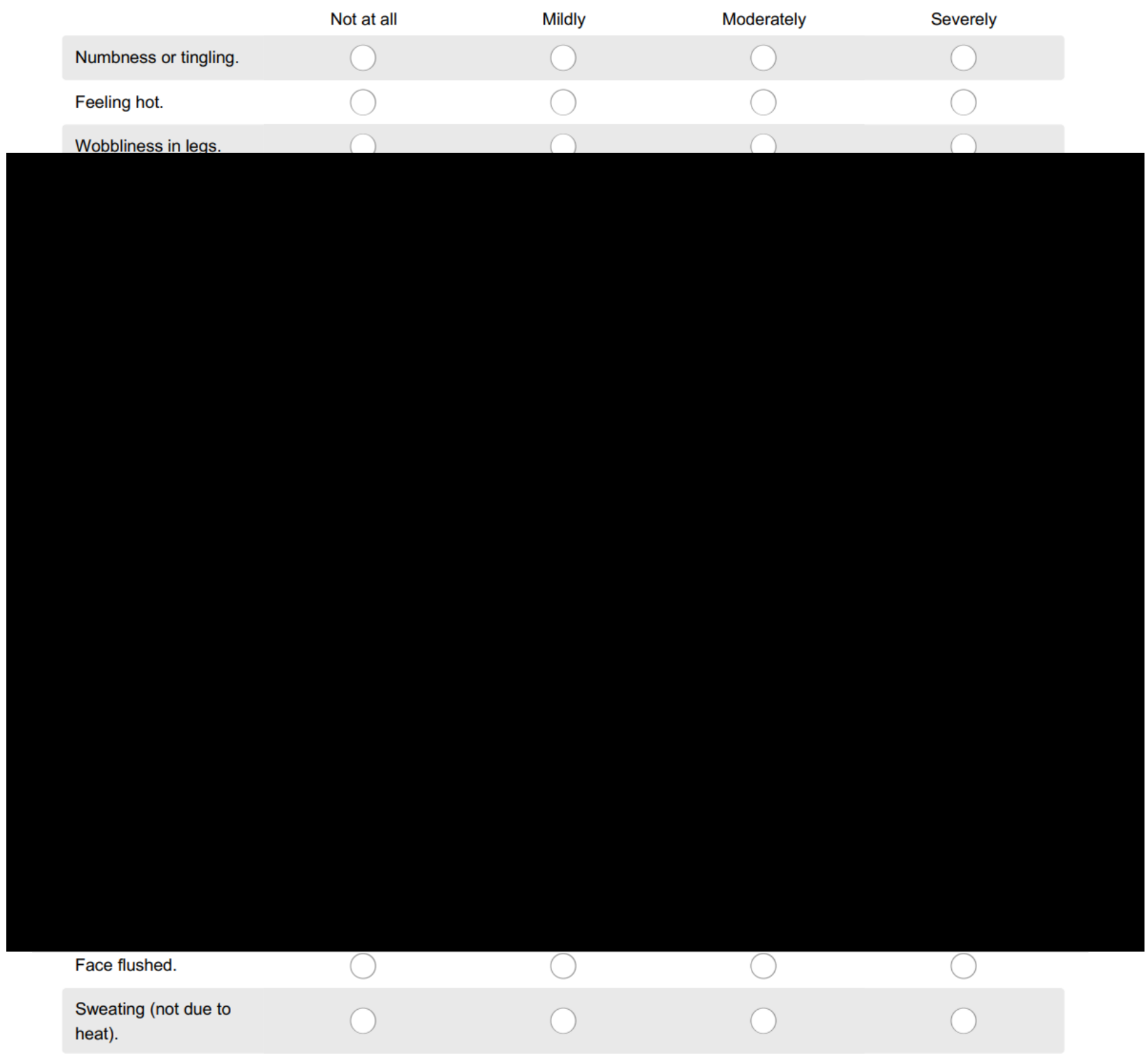




\section{Appendix H: Emotional Eating Scale}

Arnow, Kenardy, \& Agras (1995)

We all respond to different emotions in different ways. Some types of feelings lead people to experience an urge to eat. Please indicate the extent to which the following feelings lead you to feel an urge to eat by checking the appropriate box.

* 38. EES

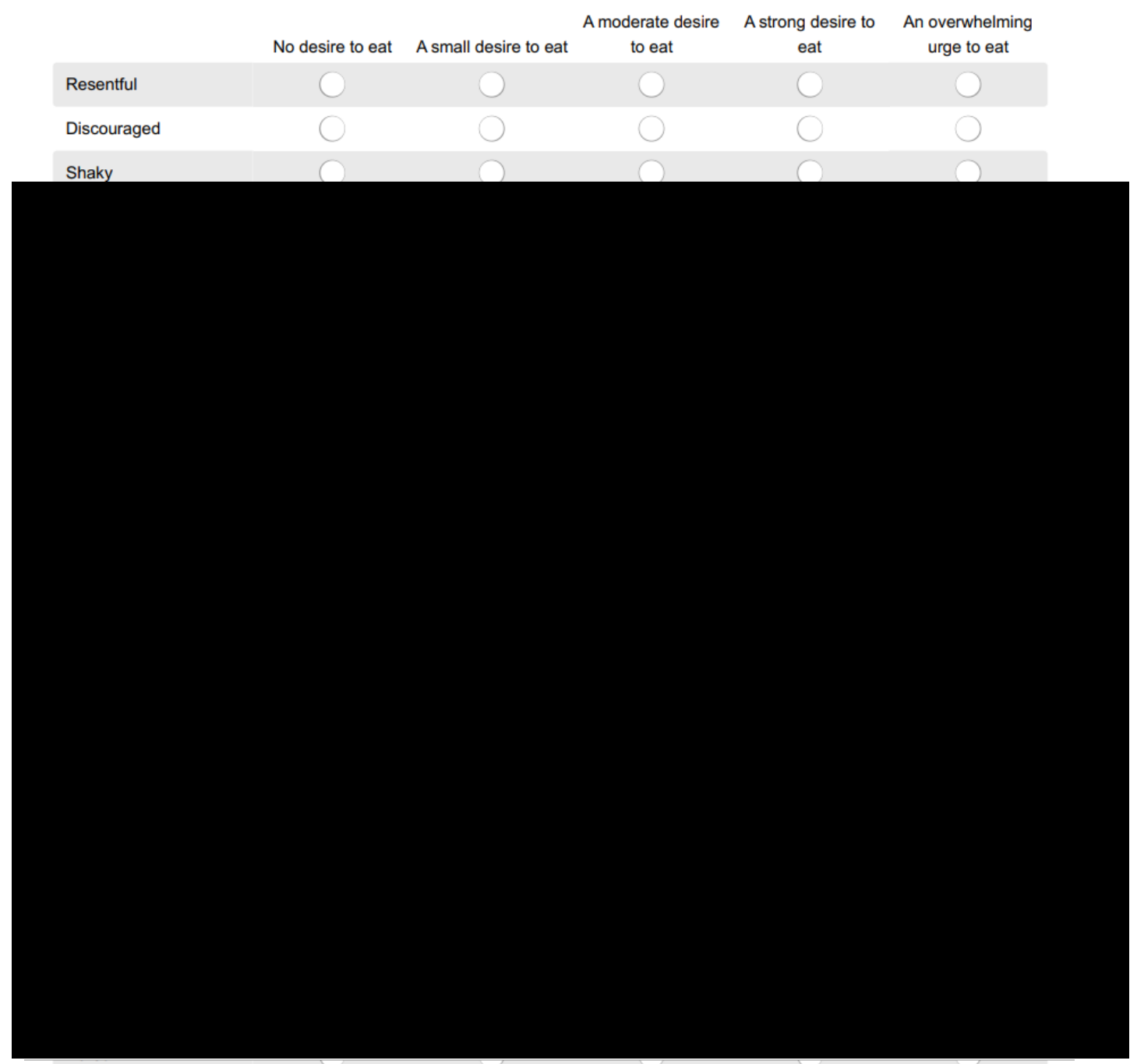

Helpless

Upset 
Appendix I. Three Factor Eating Questionnaire - R18

Karlsson, Persson, Sjöström, \& Sullivan (2000)

\section{Cognitive Restraint}

1. I deliberately take small helpings as a means of controlling my weight.

definitely true/mostly true/mostly false/definitely false

2 I consciously hold back at meals in order not to

definitely true/mostly true/mostly false/definitely

false

5. I get so hungry that my stomach often seems like a bottomless pit.

definitely true/mostly true/mostly false/definitely false 
6. I am always hungry so it is hard for me to stop eating before I finish the food on my plate.

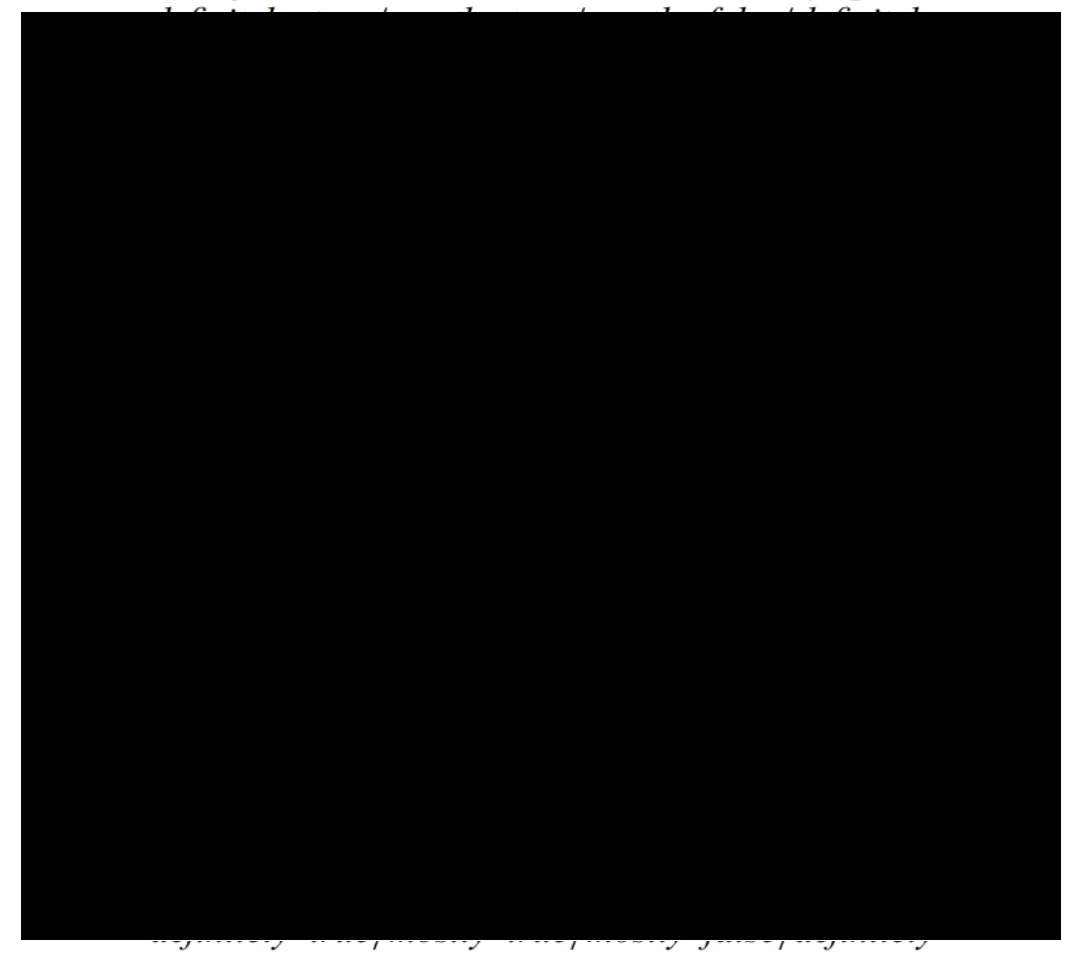

false

3. When I feel lonely, I console myself by eating. definitely true/mostly true/mostly false/definitely false 


\section{Appendix J: Correlations between alexithymia, negative affect, impulsivity, emotional eating and BMI for Study One}

\section{Correlations between alexithymia, emotional eating and BMI}

Pearson correlation analysis revealed a small, significant positive correlation between EES and TAS-20 total scores $(r=.176, p=.049)$. DIF was also significantly correlated with total EES $(r=.203, p=.024)$ and anger/frustration subscale scores $(r$ $=.197, p=.028)$. The correlation between DIF and EES depression subscale scores approached significance $(r=.171, p=.056)$, but no other significant correlations were found (see Table 1). No correlations remained significant after controlling for multiple comparisons (Bonferroni adjusted alpha level; $p=.002$ ). There was no significant correlation between BMI and emotional eating (EES: $r=.150, p=.094$; ang/frus: $r=$ $.103, p=.253$; anx: $r=.159, p=.076$; dep: $r=.164, p=.068$; TFEQ-EE: $r=.138, p=$ $.126)$.

Table 1. Pearson correlations between alexithymia, emotional eating and BMI.

\begin{tabular}{lcccccc}
\hline & Ang/Frus & Anx & Dep & EES & TFEQ-EE & BMI \\
\hline DIF & $.197^{*}$ & .167 & .171 & $.203^{*}$ & .074 & .151 \\
DDF & .050 & .094 & .104 & .085 & .018 & .078 \\
EOT & .089 & .137 & .088 & .115 & .090 & -.067 \\
TAS-20 & .150 & .170 & .159 & $.176^{*}$ & .076 & .083 \\
\hline Ang/Frus = anger/frustration EES subscale, Anx = anxiety EES subscale, BMI = body \\
mass index, DDF = difficulty describing feelings, Dep = depression EES subscale, DIF \\
$=$ difficulty identifying feelings, EES = total EES, EOT = externally oriented thinking, \\
TAS-20 = total Toronto alexithymia scale, TFEQ-EE = emotional eating TFEQ \\
subscale. $*=p<.05$.
\end{tabular}




\section{Correlations between impulsivity, emotional eating and BMI}

Significant positive correlations were found between UPPS-P and EES total ( $r$ $=.183, p=.041)$ and depression subscale scores $(r=.208, p=.020)$. Significant moderate correlations were also found between total NU and emotional eating scores as measured by EES $(r=.385, p<.001)$ and TFEQ $(r=.328, p<.001)$. In addition, total NU scores were also significantly positively correlated with EES anger and frustration $(r=.284, p=.001)$, anxiety $(r=.382, p<.001)$ and depression $(r=.414, p$ <.001) subscale scores. PU was significantly correlated with EES total $(r=.202, p=$ $.024)$ and EES anxiety subscale scores $(r=.210, p=.019)$. The lack of perseverance (Pers) subscale was significantly correlated with TFEQ-EE $(r=.188, p=.036)$ and EES depression subscale scores $(r=.180, p=.044)$. No other significant correlations were found (see Table 2). Only correlations with NU remained significant after a Bonferroni adjusted alpha level was adopted $(p \leq .001)$.

Table 2. Pearson correlations between impulsivity, emotional eating and BMI.

\begin{tabular}{|c|c|c|c|c|c|c|}
\hline & Ang/Frus & Anx & $\overline{\text { Dep }}$ & $\overline{\mathrm{EES}}$ & TFEQ-EE & BMI \\
\hline $\mathrm{NU}$ & $.284 * * *$ & $.382 * * *$ & $.414 * * *$ & $.385 * * *$ & $.328 * * *$ & .077 \\
\hline PU & .174 & $.210^{*}$ & .158 & $.202 *$ & .087 & .051 \\
\hline Prem & -.042 & -.042 & .037 & -.026 & -.003 & -.051 \\
\hline Pers & .158 & .083 & $.180^{*}$ & .156 & $.188^{*}$ & -.081 \\
\hline SS & -.082 & -.100 & -.050 & -.089 & -.067 & -.058 \\
\hline UPPS-P & .143 & .163 & $.208^{*}$ & $.183^{*}$ & .143 & -.006 \\
\hline
\end{tabular}




\section{Correlations between negative affect, emotional eating and BMI}

Significant positive correlations were found between total EES, BDI $(r=.265$, $p=.003)$ and BAI $(r=.271, p=.002)$ scores. Significant correlations were also found between BDI and EES anger/frustration $(r=.273, p=.002)$ and anxiety $(r=.255, p$ $=.004$ ) subscale scores. No significant correlation was found between BDI and EES depression subscale scores $(r=.148, p=.099)$, but significant positive correlations were found with the anger/frustration $(r=.273, p=.002)$ and anxiety $(r=.255, p$ $=.004)$ subscales. Similarly, BAI scores were significantly correlated with EES anger/frustration $(r=.286, p=.001)$ and anxiety $(r=.293, p=.001)$ subscales scores, but not depression $(r=.096, p=.287)$. No other significant correlations were found (see Table 3). With the exception of the correlation between BDI and EES total scores, all correlations remained significant after controlling for multiple comparisons (Bonferroni adjusted alpha level; $p<.004$ ).

Table 3. Pearson correlations between negative affect, emotional eating and BMI.

\begin{tabular}{ccccccc}
\hline & Ang/Frus & Anx & Dep & EES & TFEQ-EE & BMI \\
\hline BDI & $.273^{* *}$ & $.255^{* *}$ & .148 & $.265^{* *}$ & .069 & .065 \\
BAI & $.286^{* * *}$ & $.293^{* * *}$ & .096 & $.271^{* *}$ & -.037 & .144 \\
\hline
\end{tabular}

Ang/Frus = anger/frustration EES subscale, $\mathrm{Anx}=$ anxiety EES subscale, $\mathrm{BAI}=\mathrm{Beck}$ anxiety inventory, $\mathrm{BDI}=$ Beck depression inventory, $\mathrm{BMI}=$ body mass index, $\mathrm{DIF}=$ difficulty identifying feelings, Dep = depression EES subscale, EES = total EES, TFEQ-EE $=$ emotional eating TFEQ subscale. $* *=p<.01, * * *=p \leq .001$.

\section{Correlations between alexithymia and impulsivity}

Significant positive correlations were found between total TAS-20 and UPPS$\mathrm{P}(r=.344, p<.001)$ scores, as well as between TAS-20 total, NU $(r=.341, p<.001)$, PU $(r=.345, p<.001)$ and lack of perseverance (pers; $r=404, p<.001)$ subscale scores. Total UPPS-P scores were most strongly correlated with the DIF subscale of 
the TAS-20 $(r=.365, p<.001)$, but was also weakly correlated with DDF $(r=.196, p$ $=.028)$ and EOT $(r=.233, p=.009)$. There were a number of other significant correlations between the TAS-20 and UPPS-P subscales (see Table 4). Correlations with $p<.002$ remained significant after applying a Bonferroni adjustment.

Table 4. Pearson correlations between alexithymia and impulsivity.

\begin{tabular}{|c|c|c|c|c|c|c|}
\hline & $\mathrm{NU}$ & $\mathrm{PU}$ & Prem & Pers & $\mathrm{SS}$ & UPPS-P \\
\hline DIF & $.422 * * *$ & $.407 * * *$ & .094 & $.390 * * *$ & -.032 & $.365 * * *$ \\
\hline DDF & $.234 * *$ & $.196 *$ & .048 & $.251 * *$ & -.020 & $.196 *$ \\
\hline EOT & .102 & $.176^{*}$ & $.284 * * *$ & $.300 * * *$ & .036 & $.233 * *$ \\
\hline TAS-20 & $.341 * * *$ & $.345 * * *$ & .168 & $.404 * * *$ & -.011 & $.344 * * *$ \\
\hline
\end{tabular}


Appendix K. Study Two: Ethics Memo

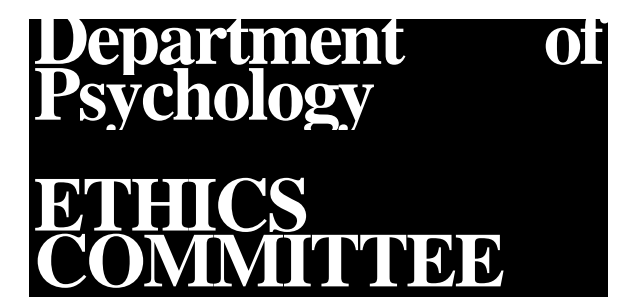

\section{Memo}

To:

Aimee Pink

From: Dr. Jeremy Tree

for Departmental Ethics Committee

Copy: $\quad$ Dr. Claire Williams

Date: $\quad 1^{\text {th }}$ September, 2015

Re: Investigating the relationships between alexithymia, impulsivity and emotional eating

Your proposed study "Investigating the relationships between alexithymia, impulsivity and emotional eating", has been reviewed and is approved. Provided that the information obtained is kept absolutely confidential and that no personally identifiable information is entered on computer, you may proceed with your studies.

Please ensure that the signed copy of this Ethical Approval, together with any other paperwork associated with your research, is included in your final write up.

In order for your study to be displayed on the Experiment Management System (Participant Pool):

Forward this approval via email to Dr. Irene Reppa (

$\underline{\text { AND }}$

Send a request for your study to be made visible, via the link on the EMS website (see Researcher Documentation for details). 
Appendix L: Correlations between alexithymia, negative affect, impulsivity, emotional eating and BMI for Study Two

\section{Correlations between alexithymia, emotional eating and BMI.}

TAS-20, DIF and DDF were significantly and positively correlated with emotional eating as measured by the EES (TAS-20: $r=.217, p<.001$; DIF: $r=.265$, $p<.001$; and DDF: $r=.174, p=.001$ ) and TFEQ (TAS-20: $r=.135, p=.012$; DIF: $r$ $=.180, p=.001$; DDF: $r=.128, p=.018)$. Significant correlations were also found between TAS-20 and the EES subscales scores (see Table 1). Self-reported BMI was significantly correlated with all aspects of emotional eating (ang/frus: $r=213, p<$ .001 , anx: $r=.164, p=.002$, dep: $r=.206, p<.001$, EES: $r=212, p<.001$, and TFEQ-EE: $r=.2, p<.001)$. All significant correlations at $p \leq .001$ remained significant after applying a Bonferroni adjusted alpha level to control for inflated type 1 error $(p$ $=.002$, see Table 11).

Table 1. Pearson correlations between alexithymia, emotional eating and BMI.

\begin{tabular}{lcccccc}
\hline & Ang/Frus & Anx & Dep & EES & TFEQ-EE & BMI \\
\hline DIF & $.254 * * *$ & $.262 * * *$ & $.201 * * *$ & $.265^{* * *}$ & $.180^{* * *}$ & .050 \\
DDF & $.157 * *$ & $.196 * * *$ & $.119^{*}$ & $.174 * * *$ & $.128^{*}$ & -.025 \\
EOT & .066 & .066 & .010 & .058 & -.004 & .002 \\
TAS-20 & $.208 * * *$ & $.227 * * *$ & $.147 * *$ & $.217 * * *$ & $.135 *$ & .016 \\
\hline Ang/Frus = anger/frustration EES subscale, Anx = anxiety EES subscale, BMI = body \\
mass index, DDF = difficulty describing feelings, Dep = depression EES subscale, DIF \\
$=$ difficulty identifying feelings, EES = total EES, EOT = externally oriented thinking, \\
TAS-20 = total Toronto alexithymia scale, TFEQ-EE = emotional eating TFEQ \\
subscale. $*=p \leq .05, * *=p \leq .01, * * *=p \leq .001$
\end{tabular}




\section{Correlations between impulsivity, emotional eating and BMI}

Significant positive correlations were found between total UPPS-P and emotional eating scores (EES: $r=.224, p<.001$ : TFEQ: $r=.150, p<.001$ ). Total UPPS-P scores were significantly correlated with all subscales of the EES (ang/frus: $r$ $=.215, p<.001$; anx: $r=.217, p<.001$; dep: $r=.175, p=.001)$, and significant correlations were also found between each of the UPPS-P and EES subscales (see Table 12). EES total scores were also significantly correlated with NU ( $r=.350, p$ $<.001)$, Pers $(r=.237, p<.001)$ and PU $(r=.159, p=.003)$, and with the exception of PU, a similar pattern of results was found for the TFEQ-EE (see Table 2). BMI was significantly correlated with NU $(r=.152, p=.005)$, Pers $(r=.141, p=.009)$ and SS $(r=-.138, p=.010)$. After applying Bonferroni corrected alpha $(p=.001)$, all correlations that were significant to $p \leq .001$ remained significant.

Table 2. Pearson correlations between impulsivity and emotional eating.

\begin{tabular}{lcccccc}
\hline & Ang/Frus & Anx & Dep & EES & TFEQ-EE & BMI \\
\hline NU & $.321 * * *$ & $.321 * * *$ & $.333^{* * *}$ & $.350^{* * *}$ & $.324 * * *$ & $.152^{* *}$ \\
PU & $.146^{* *}$ & $.163 * *$ & $.124 *$ & $.159 * *$ & .082 & .003 \\
Prem & .102 & .077 & .026 & .083 & .017 & .040 \\
Pers & $.242^{* * *}$ & $.216^{* * *}$ & $.179 * * *$ & $.237 * * *$ & $.160 * *$ & $.141 * *$ \\
SS & -.027 & -.001 & -.035 & -.022 & -.040 & $-.138^{*}$ \\
UPPS-P & $.215^{* * *}$ & $.217 * * *$ & $.175^{* *}$ & $.224 * * *$ & $.150^{* *}$ & .044 \\
\hline Ang/Frus & & & & &
\end{tabular}

Ang/Frus = anger/frustration EES subscale, Anx = anxiety EES subscale, BMI = body mass index, Dep = depression EES subscale, EES = total EES, NU = negative urgency, Pers $=($ lack of $)$ perseverance, Prem $=($ lack of $)$ premeditation, $P U=$ positive urgency, $\mathrm{SS}=$ sensation seeking, TFEQ-EE = emotional eating TFEQ subscale, UPPS-P Total $=$ total UPPS-P. $*=p \leq .05, * *=p \leq .01, * * *=p \leq .001$. 


\section{Correlations between negative affect, emotional eating and BMI}

Significant positive correlations were found between BDI and emotional eating scores (EES: $r=.371, p<.001$ : TFEQ: $r=.219, p<.001$ ). BAI was significantly correlated with EES $(r=.241, p<.001)$, but not TFEQ-EE $(r=.093, p=.085)$ scores. BDI and BAI scores were also significantly correlated with the subscales of the EES (see Table 3). A weak significant correlation was found between BDI and self-reported BMI $(r=.130, p=.016)$. After applying Bonferroni corrected alpha $(p=.004)$, all correlations that were significant to $p \leq .001$ remained significant.

Table 3. Associations between negative affect and emotional eating.

\begin{tabular}{ccccccc}
\hline & Ang/Frus & Anx & Dep & EES & TFEQ-EE & BMI \\
\hline BDI & $.307 * * *$ & $.321 * * *$ & $.227 * * *$ & $.317 * * *$ & $.219 * * *$ & $.130 *$ \\
BAI & $.248 * * *$ & $.227 * * *$ & $.169 * *$ & $.241 * * *$ & .093 & -.016 \\
\hline
\end{tabular}

Ang/Frus = anger/frustration EES subscale, Anx = anxiety EES subscale, BAI = Beck anxiety inventory, BDI = Beck depression inventory, $\mathrm{BMI}=$ body mass index, Dep = depression EES subscale, EES = total EES, TFEQ-EE = emotional eating TFEQ subscale. $*=p \leq .05, * *=p \leq .01, * * *=p \leq .001$.

\section{Correlations between alexithymia and impulsivity}

Negative urgency was significantly correlated with alexithymia total and subscale scores (DIF: $r=.524, p<.001$, DDF: $r=.396, p<.001$, EOT: $r=.214, p<$ .001 , TAS-20 total: $r=.485, p<.001)$. Total UPPS-P, PU, Prem and Pers scores were also significantly correlated alexithymia total and subscale scores (see Table 4). All correlations remained significant after applying a Bonferroni correction $(p=.002)$. 
Table 4. Pearson correlations between alexithymia and impulsivity.

\begin{tabular}{|c|c|c|c|c|c|c|}
\hline & $\mathrm{NU}$ & $\mathrm{PU}$ & Prem & Pers & $\mathrm{SS}$ & UPPS-P \\
\hline$\overline{\mathrm{DIF}}$ & $.524 * * *$ & $.321 * * *$ & .041 & $.33 * * *$ & .02 & $.352 * * *$ \\
\hline DDF & $.396 * * *$ & $.294 * * *$ & .02 & $.229 * * *$ & .091 & $.294 * * *$ \\
\hline EOT & $.214 * * *$ & $.246^{* * * *}$ & $.177 * * *$ & $.175^{* * * *}$ & .075 & $.247 * * *$ \\
\hline TAS-20 & $.485 * * *$ & $.352 * * *$ & .092 & $.312 * * *$ & .071 & $.376^{* * * *}$ \\
\hline
\end{tabular}


Appendix M. Study Three: Ethics Memo

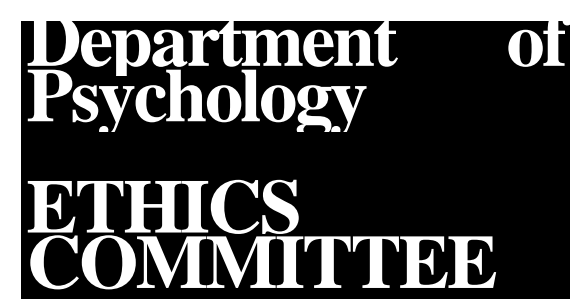

\section{Memo}

To:

Aimee Pink

From: Dr. Jeremy Tree

for Departmental Ethics Committee

Copy: $\quad$ Dr. Claire Williams

Date: $\quad 1^{\text {th }}$ March, 2016

Re: $\quad$ Pilot study of autobiographical recall as a method of mood induction

Your proposed study "Pilot study of autobiographical recall as a method of mood induction", has been reviewed and is approved. Provided that the information obtained is kept absolutely confidential and that no personally identifiable information is entered on computer, you may proceed with your studies.

Please ensure that the signed copy of this Ethical Approval, together with any other paperwork associated with your research, is included in your final write up.

In order for your study to be displayed on the Experiment Management System (Participant Pool):

Forward this approval via email to Dr. Phil Tucker

\section{$\underline{\text { AND }}$}

Send a request for your study to be made visible, via the link on the EMS website (see Researcher Documentation for details). 


\section{Appendix N. Profile of Moods Scale}

McNair, Lorr \& Droppleman (1971)

Below are words that describe feelings and moods people have. Please read EVERY word carefully. Then select the answer which best describes how you have been feeling TODAY, at this moment.

Suppose the word is "happy", mark the one answer which is closest to how you have been feeling TODAY.

* 9. Please read carefully.

Lively

Composed

Angry

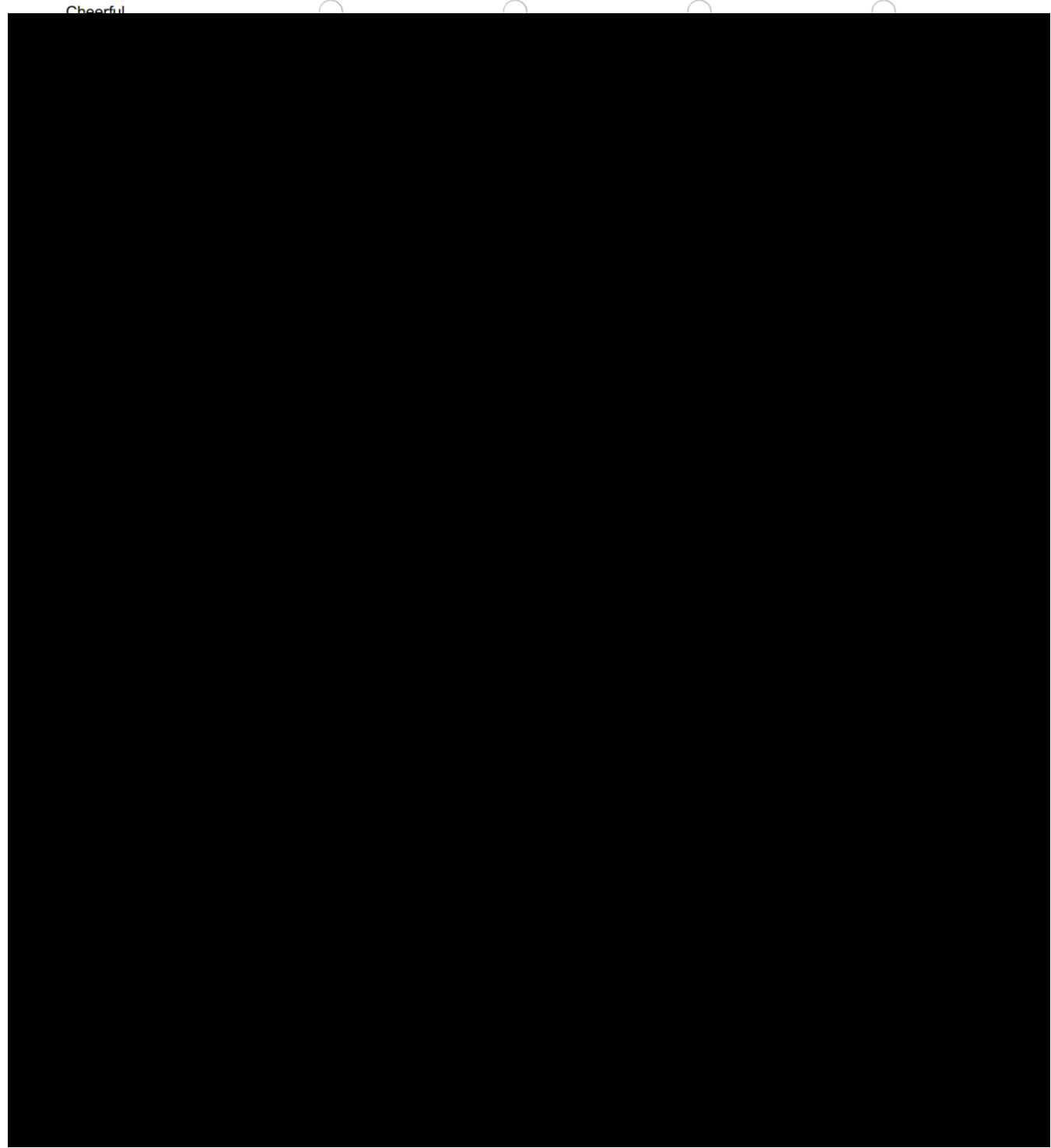

Energetic

Lonely

Sympathetic

Exhausted 


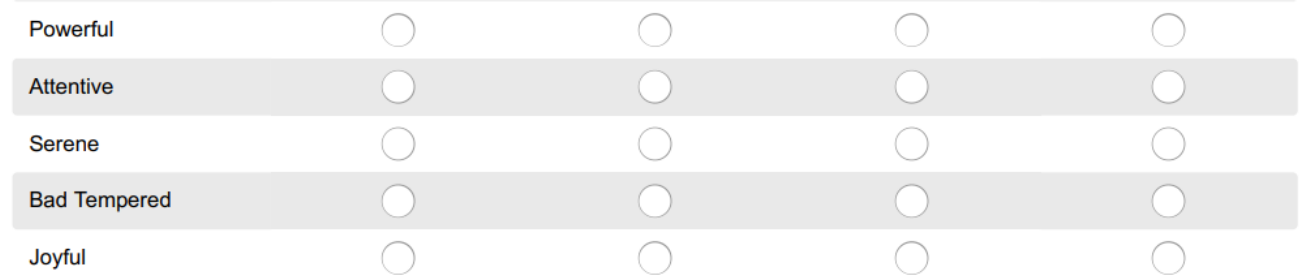

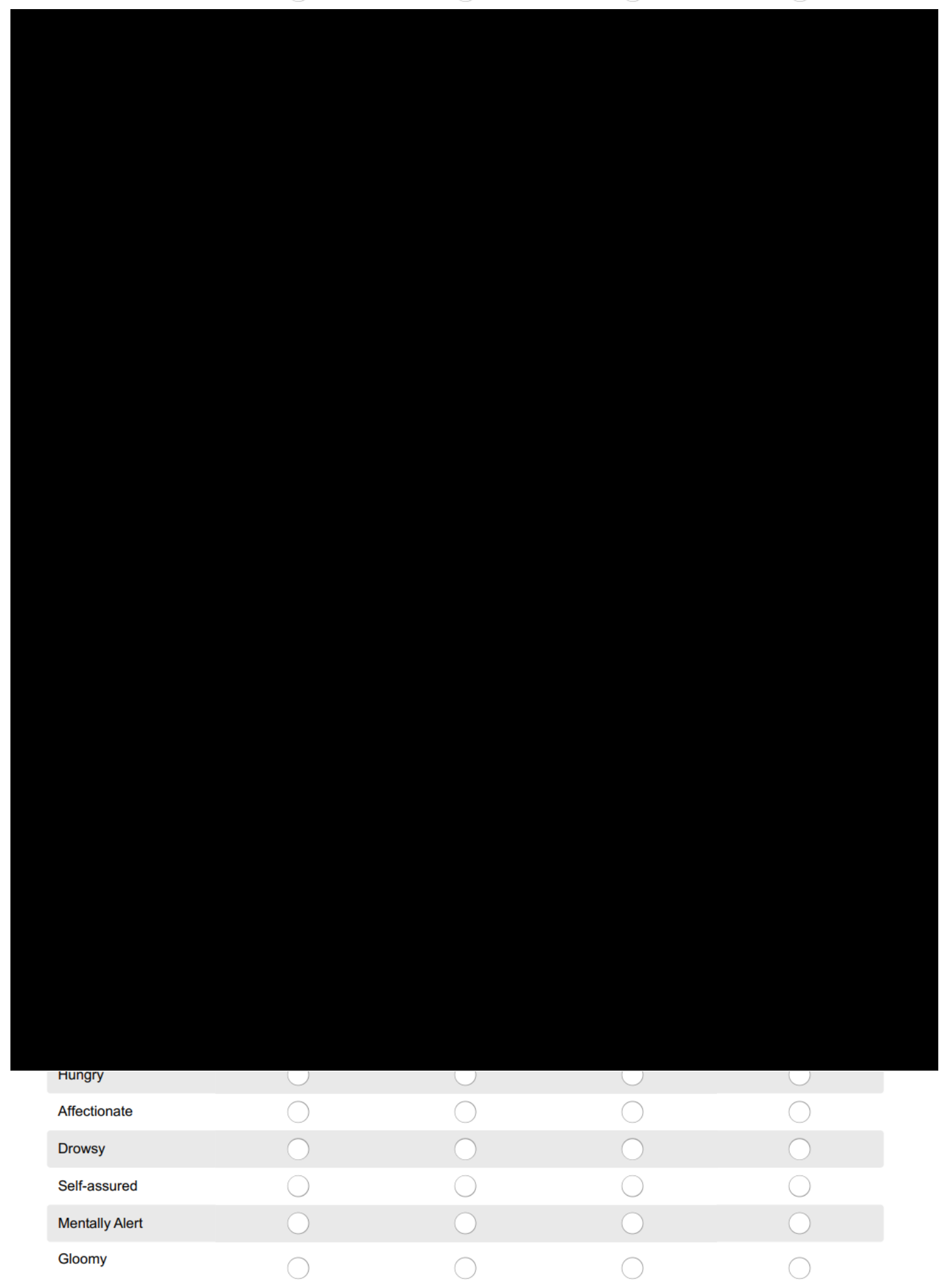

404 | P a g e 


\section{Appendix O. Subjective measure of Mixed Emotion}

Berrios, Totterfell, \& Kellett (2015b)

* 10. Please read carefully and answer with the response that best describes how you are feeling TODAY, at this moment.

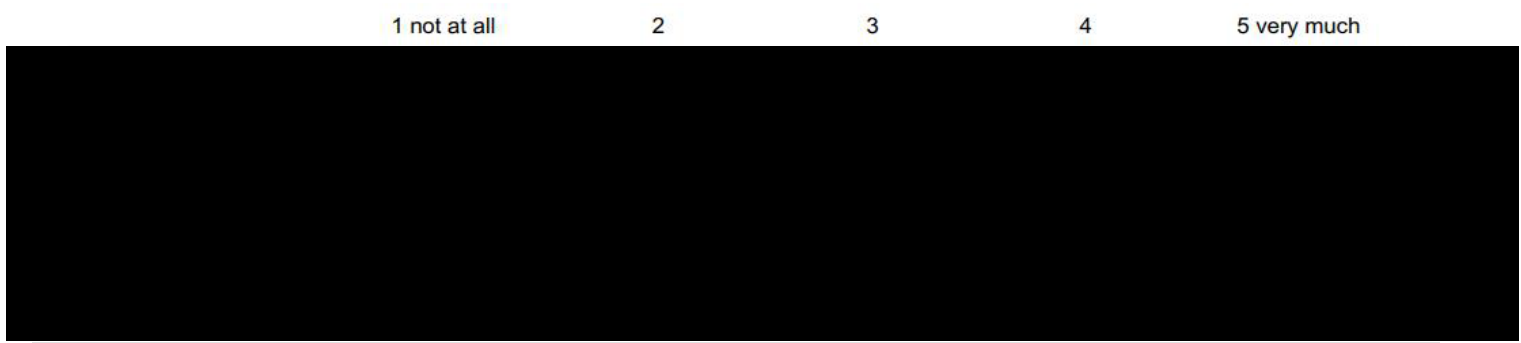

I'm feeling a combination

of different emotions at

the same time 


\section{Appendix P. Memory Characteristics Questionnaire}

Johnson, Foley, Suengas, \& Raye (1988)

* 36. My memory for this event is. .

$1 \mathrm{dim}$ 2 
* 45. Relative spatial arrangement of objects in my memory for the event is. .

\begin{tabular}{lllllll}
1 vague & 2 & 3 & 4 & 5 & 6 & 7 clear/distinct \\
\hdashline & 0 & & & & &
\end{tabular}

* 46. Relative spatial arrangement of people in my memory for this event is. .
1 vague
3
7 clear/distinct

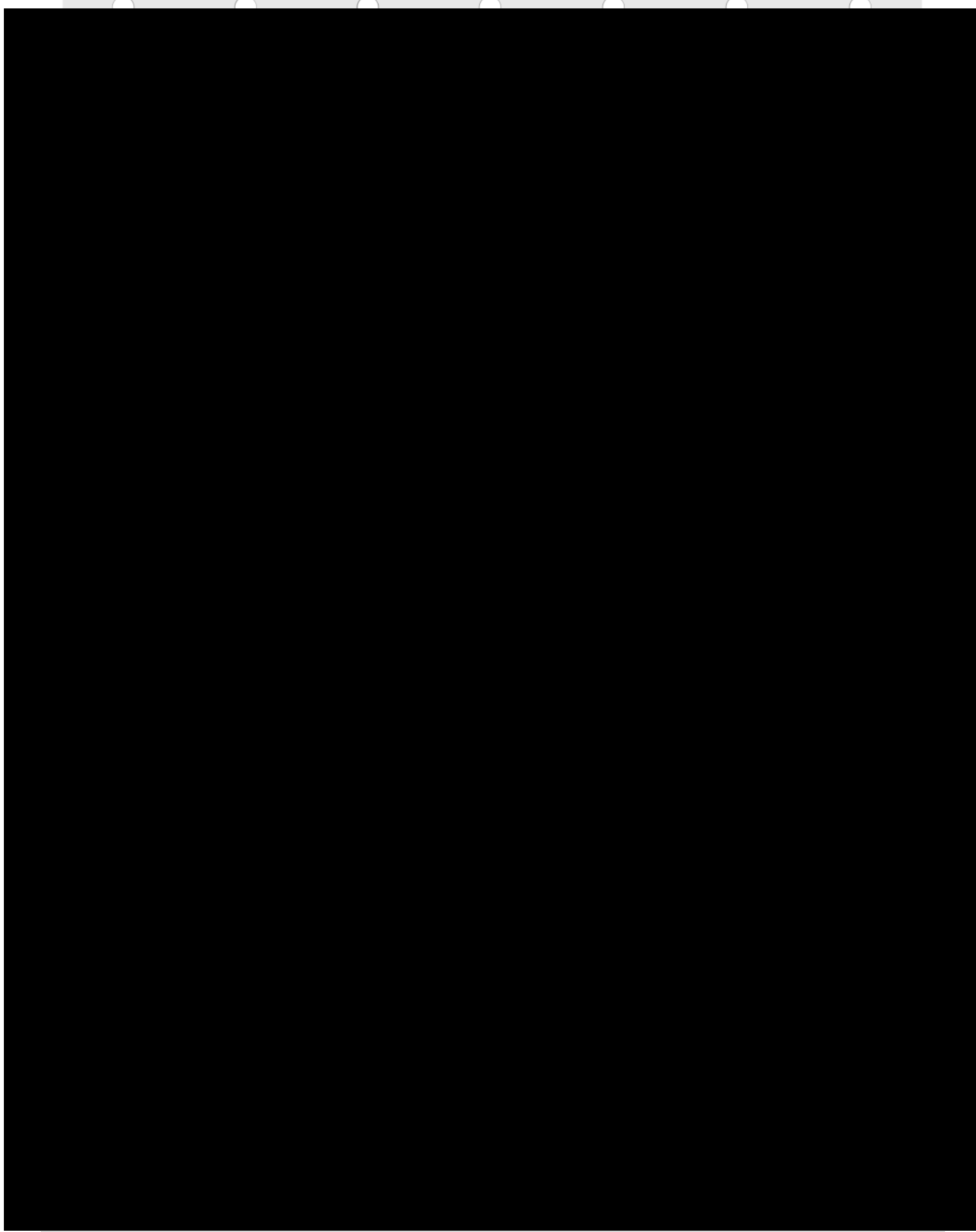

* 54 . Feelings at the time were. .

1 negative

3 
* 55. Feelings at the time were. . .

1 not intense

2

3

7 very intense

* 56. As I am remembering now, my feelings are. .

* 62. Since it all happened, I have thought about this event.

1 not at all 
* 63. About when did this event happen?

Just today

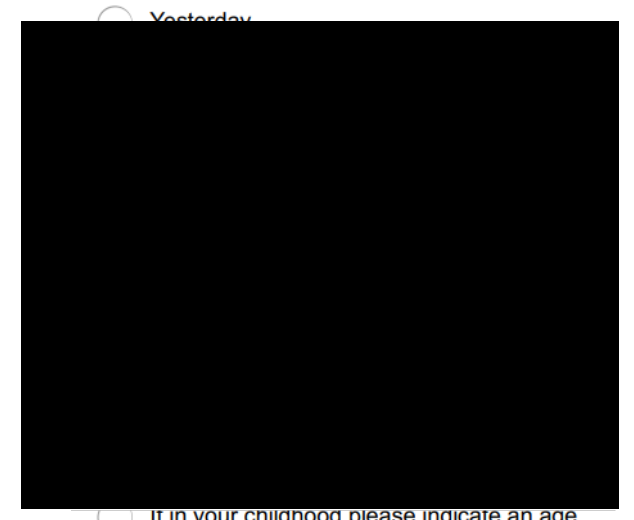

Other (please specify) Age

409 |P a g e 


\section{Appendix Q. Study Three: Mood Manipulation Instructions}

Positive: During the next 7 minutes, I would like you to think about an event in which you have personally felt happy. I would like you to recall and write about such an event as vividly and in as much detail as possible.

I would like you to picture the event happening again to you and immerse yourself in the thoughts you had at the time. Also, think about what led up to that particular situation and how you felt about it and the outcome. Anything you write down will remain anonymous and confidential, so feel free to write what you like to help you fully re-experience how you felt at the time.

Negative: During the next 7 minutes, I would like you to think about an event in which you have personally felt sad. I would like you to recall and write about such an event as vividly and in as much detail as possible.

I would like you to picture the event happening again to you and immerse yourself in the thoughts you had at the time. Also, think about what led up to that particular situation and how you felt about it and the outcome. Anything you write down will remain anonymous and confidential, so feel free to write what you like to help you fully re-experience how you felt at the time.

Emotionally Confusing: Being in touch with our emotions is essential. During the next 7 minutes, I would like you to recall an event in which you have personally experienced a state of emotional confusion. By this, I mean an occasion where you have had difficulty identifying how you were feeling and have had difficulty in expressing your feelings to others in response to that event. This can include not being able to find the right words for how you are feeling or experiencing very mixed emotions where you have had difficulty explaining the complexity of such an emotional state. I would like you to recall and write about such an event as vividly and in as much detail as possible.

I would like you to picture the event happening again to you and immerse yourself in the thoughts you had at the time. Also, think about what led up to that particular situation and how you felt about it and the outcome. Anything you write down will 
remain anonymous and, so feel free to write what you like to help you fully reexperience how you felt at the time.

Control: During the next 7 minutes, I would like you to recall and write about your usual journey to either university or work. This should include the usual timeline and route you take. I would like you to picture yourself making this journey and write about it as if describing it to someone else.

Anything you write down will remain anonymous and confidential, so feel free to write what you like to help you describe your journey. 


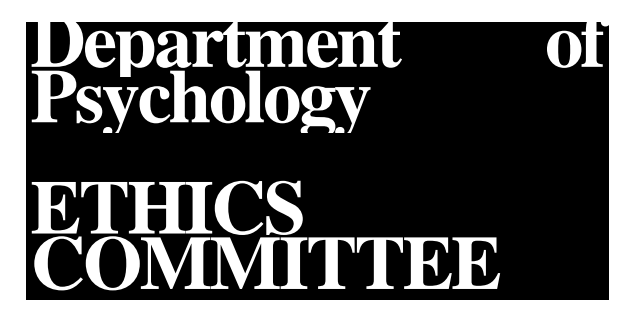

\section{Memo}

To:

Aimee Pink

From: Dr. Jeremy Tree

for Departmental Ethics Committee

Copy: Dr. Claire Williams

Date: $\quad 24^{\text {th }}$ June, 2016

Re: $\quad$ Food intake following a mood induction

Your proposed study "Food intake following a mood induction", has been reviewed and is approved. Provided that the information obtained is kept absolutely confidential and that no personally identifiable information is entered on computer, you may proceed with your studies.

Please ensure that the signed copy of this Ethical Approval, together with any other paperwork associated with your research, is included in your final write up.

In order for your study to be displayed on the Experiment Management System (Participant Pool):

Forward this approval via email to Dr. Phil Tucker

\section{AND}

Send a request for your study to be made visible, via the link on the EMS website (see Researcher Documentation for details). 


\section{Appendix S. Study Four: Mood Manipulation Instructions}

Positive: I would like you to think about an event in which you have personally felt happy. By this I mean an occasion where you have felt particularly elated. I would like you to recall and write about such an event as vividly and in as much detail as possible.

I would like you to picture yourself in your memory an you experiencing it again. Please immerse yourself in the thoughts and feelings of this event. Think about the people involved, what sounds there were and the thoughts you had at the time. Also, think about what led up to that particular situation and how you felt about it and the outcome. Anything you write down will remain anonymous and confidential and will be destroyed after the study is complete, so feel free to write what you like to help you fully re-experience how you felt at the time. Please take your time to check your memory and think about it, you will be given 10 minutes but if you feel you have finished beforehand please let me know.

Negative: During the next 7 minutes, I would like you to think about an event in which you have personally felt sad. By this I mean an occasion where you have felt particularly upset. I would like you to recall and write about such an event as vividly and in as much detail as possible.

I would like you to picture yourself in your memory an you experiencing it again. Please immerse yourself in the thoughts and feelings of this event. Think about the people involved, what sounds there were and the thoughts you had at the time. Also, think about what led up to that particular situation and how you felt about it and the outcome. Anything you write down will remain anonymous and confidential and will be destroyed after the study is complete, so feel free to write what you like to help you fully re-experience how you felt at the time. Please take your time to check your memory and think about it, you will be given 10 minutes but if you feel you have finished beforehand please let me know. 
Emotionally Confusing: Being in touch with our emotions is essential. I would like you to recall an event in which you have personally experienced a state of emotional confusion. By this, I mean an occasion where you have had difficulty identifying how you were feeling and have had difficulty in expressing your feelings to others in response to that event. This can include not being able to find the right words for how you are feeling or experiencing very mixed emotions where you have had difficulty explaining the complexity of such an emotional state. I would like you to recall and write about such an event as vividly and in as much detail as possible.

I would like you to picture yourself in your memory an you experiencing it again. Please immerse yourself in the thoughts and feelings of this event. Think about the people involved, what sounds there were and the thoughts you had at the time. Also, think about what led up to that particular situation and how you felt about it and the outcome. Anything you write down will remain anonymous and confidential and will be destroyed after the study is complete, so feel free to write what you like to help you fully re-experience how you felt at the time. Please take your time to check your memory and think about it, you will be given 10 minutes but if you feel you have finished beforehand please let me know.

Control: I would like you to recall and write about your usual journey to either university or work. This should include the usual timeline and route you take. I would like you to picture yourself making this journey and write about it as if describing it to someone else.

Anything you write down will remain anonymous and confidential and will be destroyed after the study is complete, so feel free to write what you like to help you describe your journey. 


\section{Appendix T. Study Four: Bogus Taste Test Snacks}

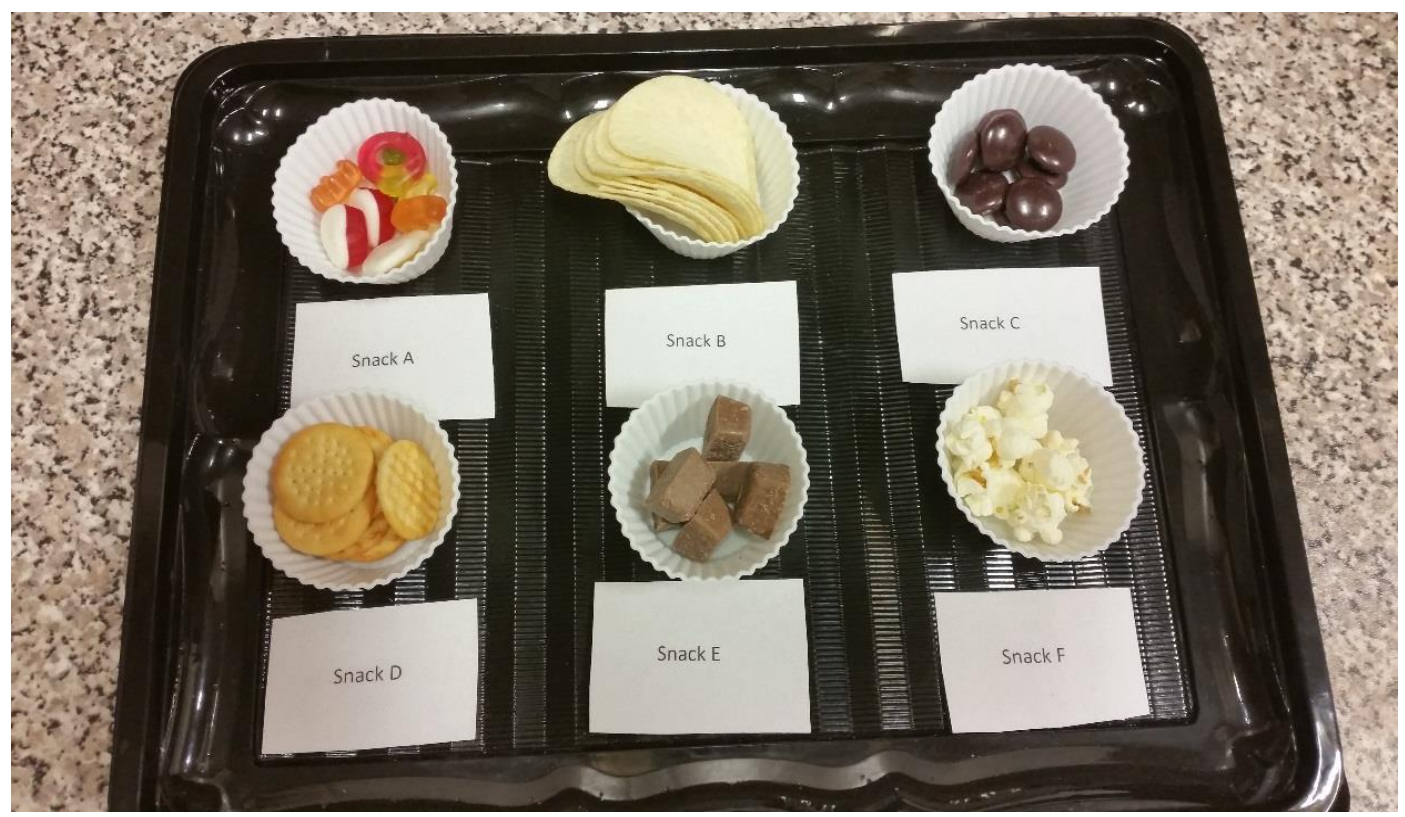

Snack A: Haribo® Starmix ${ }^{\circledR}$ (Haribo, Bonn, Germany): Roughly 8 sweets

Snack B: Pringles ${ }^{\circledR}$ Original (Kellogg's, Michigan US): 8 Pringles

Snack C: Galaxy® Minstrels ${ }^{\circledR}$ (Mars Incorporated, Virginia, US): 8 Minstrels

Snack D: Jacob's ${ }^{\circledR}$ Mini Cheddars ${ }^{\circledR}$ (McVitie’s, Edinburgh, UK): 8 Mini Cheddars

Snack E: Bitsa Wispa ${ }^{\circledR}$ (Cadbury (Mondelez, International) London, UK): 6 Bitsa Wipsa

Snack F: Tesco® Salted Popcorn (Tesco, Hertfordshire, UK): Fill the case 


\section{Appendix U. Taste Perception Task}

Example Taste Perception Task from Snack A.

Please read the following carefully.

* 38. Please complete with SNACK A in mind

Not very

much

Extremely

How much do you like

this snack?

* 39. Please complete with SNACK A in mind

Not very

Very

appealing

appealing

How appealing is the

overall taste of this

snack?

* 40. Please complete with SNACK A in mind

Not very

pleasant

pleasant

How pleasant is the

overall taste of this

snack?

* 41. Please complete with SNACK A in mind

Not very

Very

strong

Strong

How strong is the flavour

of this snack?

* 42. Please complete in SNACK A in mind

Not very

Very

healthy

healthy

How healthy do you

perceive this snack to

be?

* 43. Please answer with SNACK A in mind

Never

Very

do you eat this

snack? 
Appendix V. Study Four: Procedure Flow Diagram

Time 1

1. Demographics, POMS1, SME1, BDI, BAI

2. Mood Induction 1

3. POMS2, SME2

4. Taste Perception Task

5. POMS3, SME3, MCQ1

Time 2

6. POMS4, SME4

7. Mood Induction 2

8. POMS5, SME5

9. Taste Perception Task

10. POMS6, SME6, MCQ2, TAS-20, UPPS-P, EES, TFEQ 


\section{Appendix W. Study Four Manipulation Check}

Graphs to representing the changes in mood at baseline, post-mood and post-food for the total POMS, POMS subscales and the SME.
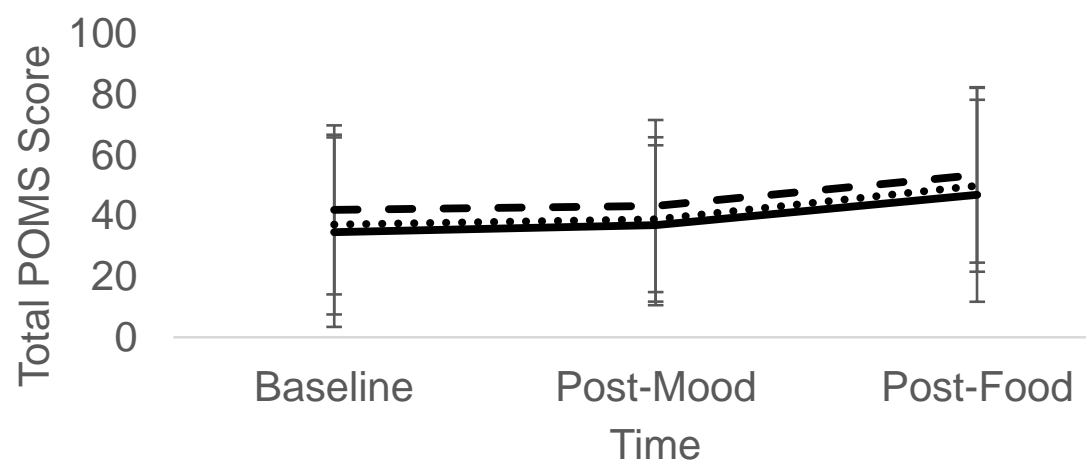

-Pos. $\quad-$ Neg. $\cdots . .$. Emo.Con.

Figure 1. Total POMS scores at baseline, post-mood and post-food for each condition during the control manipulation.

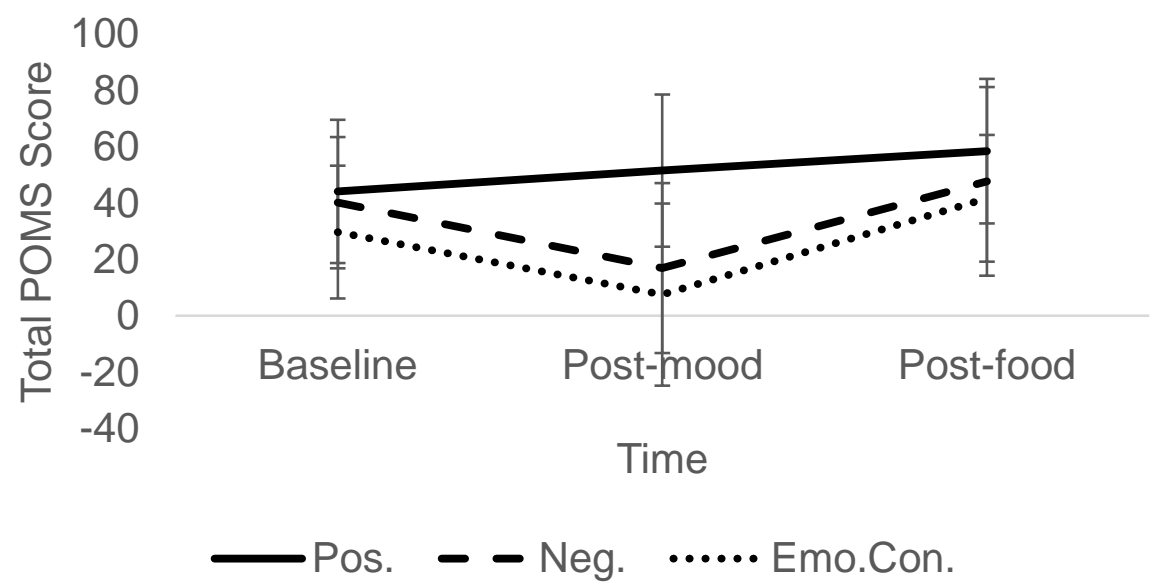

Figure 2. Total POMS scores at baseline, post-mood and post-food for each condition during the experimental mood manipulation. 


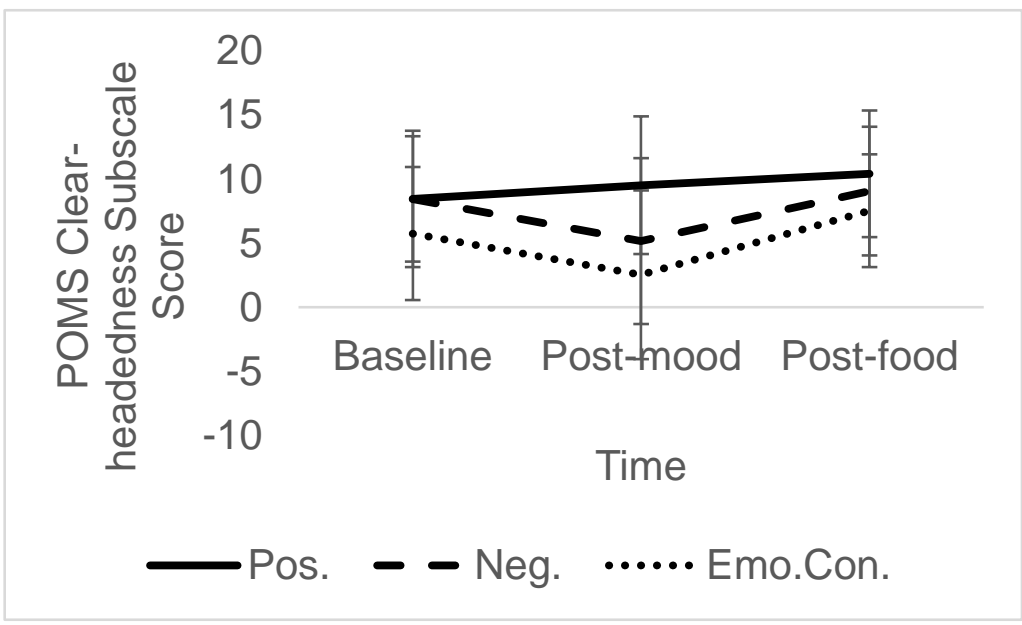

Figure 3. POMS clear-headedness scores at baseline, post-mood and post-food for each condition during the experimental mood manipulation.

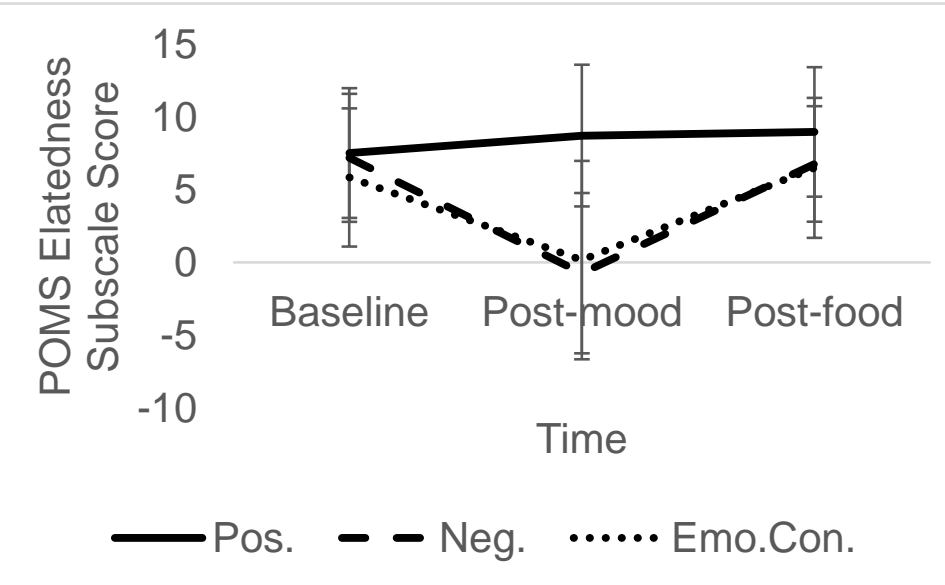

Figure 4. POMS elatedness scores at baseline, post-mood and post-food for each condition during the experimental mood manipulation. 


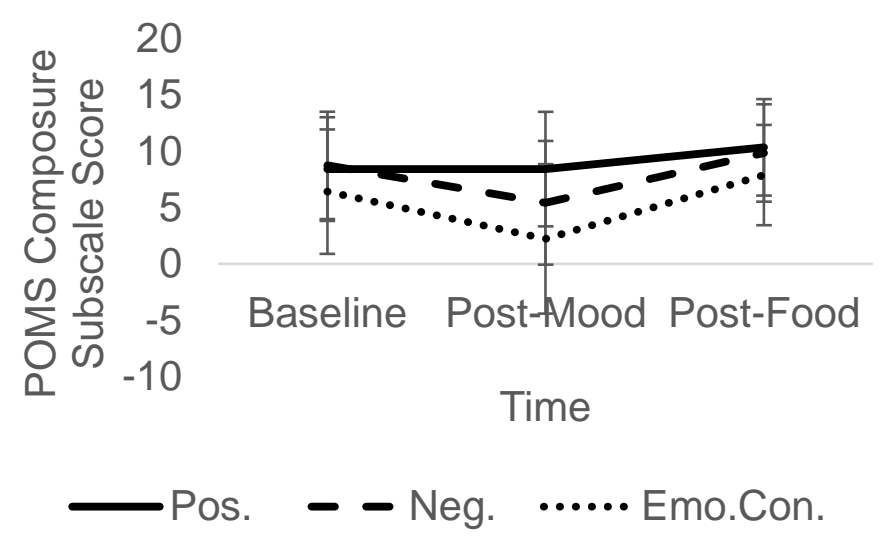

Figure 5. POMS composure scores at baseline, post-mood and post-food for each condition during the experimental mood manipulation.

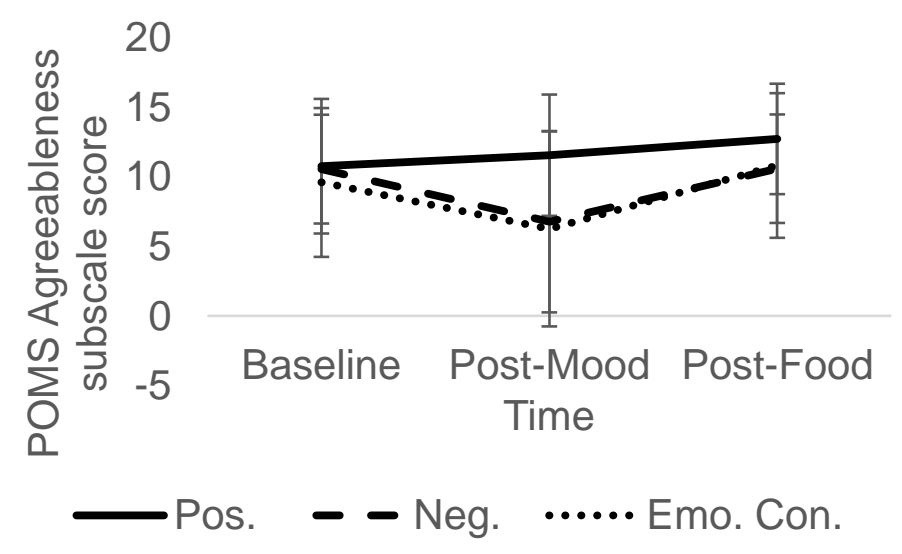

Figure 6. POMS agreeableness scores at baseline, post-mood and post-food for each condition during the experimental mood manipulation. 


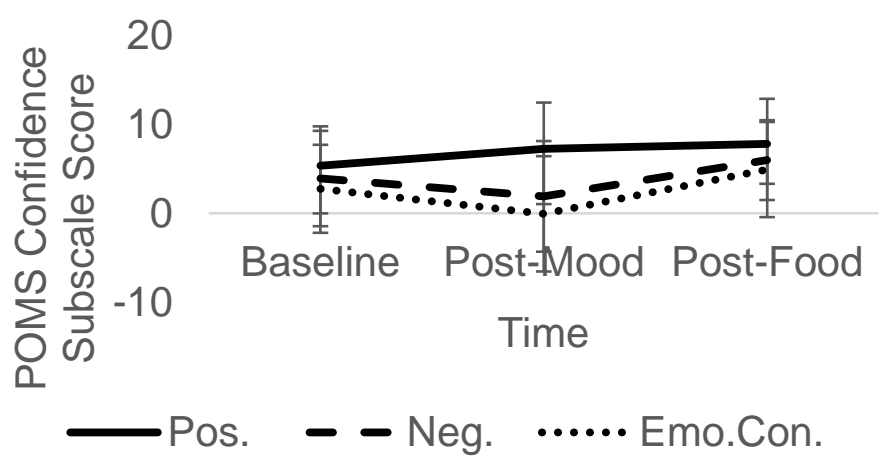

Figure 7. POMS confidence scores at baseline, post-mood and post-food for each condition during the experimental mood manipulation.

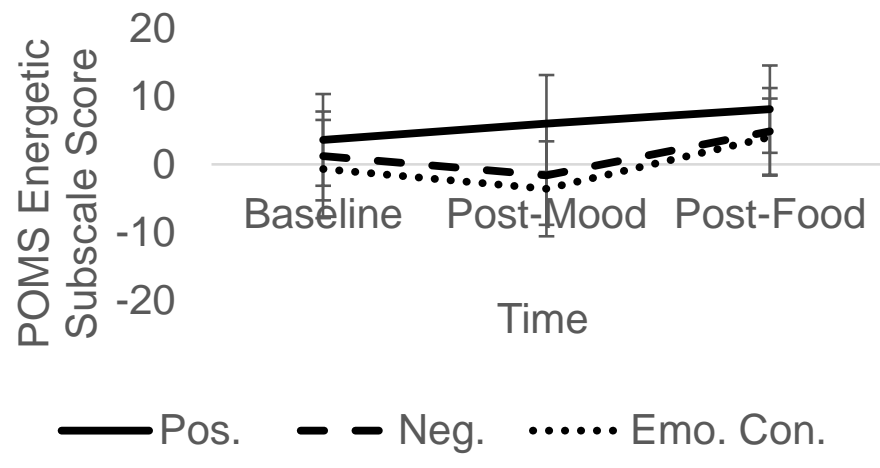

Figure 8. POMS energetic scores at baseline, post-mood and post-food for each condition during the experimental mood manipulation. 


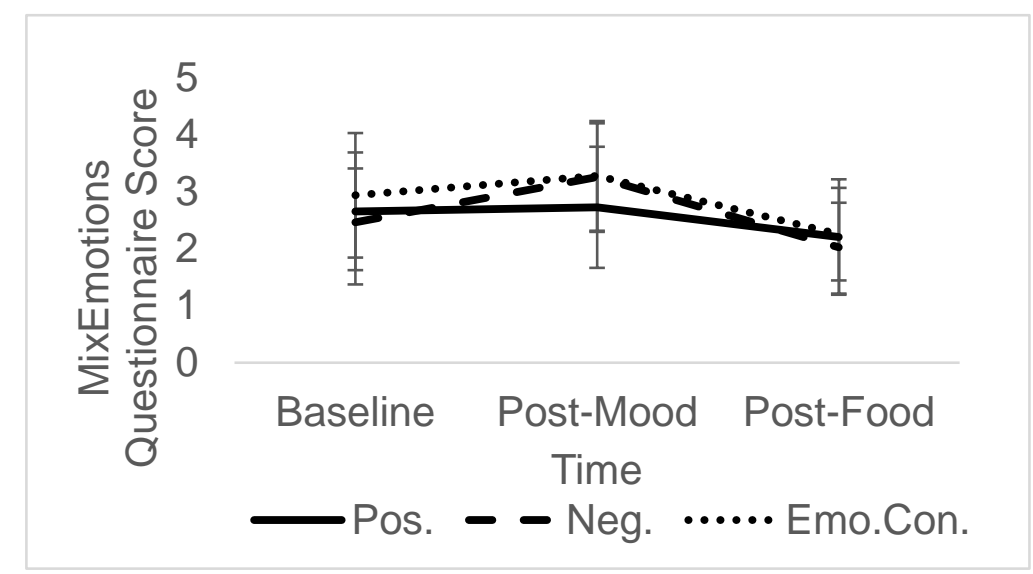

Figure 9. SME scores at baseline, post-mood and post-food for each condition during the experimental mood manipulation. 


\section{Appendix X. Dual Pathway Models}

Dual pathway model of overeating by Stice $(1994 ; 2001)$. Taken from van Strien et al. (2005).

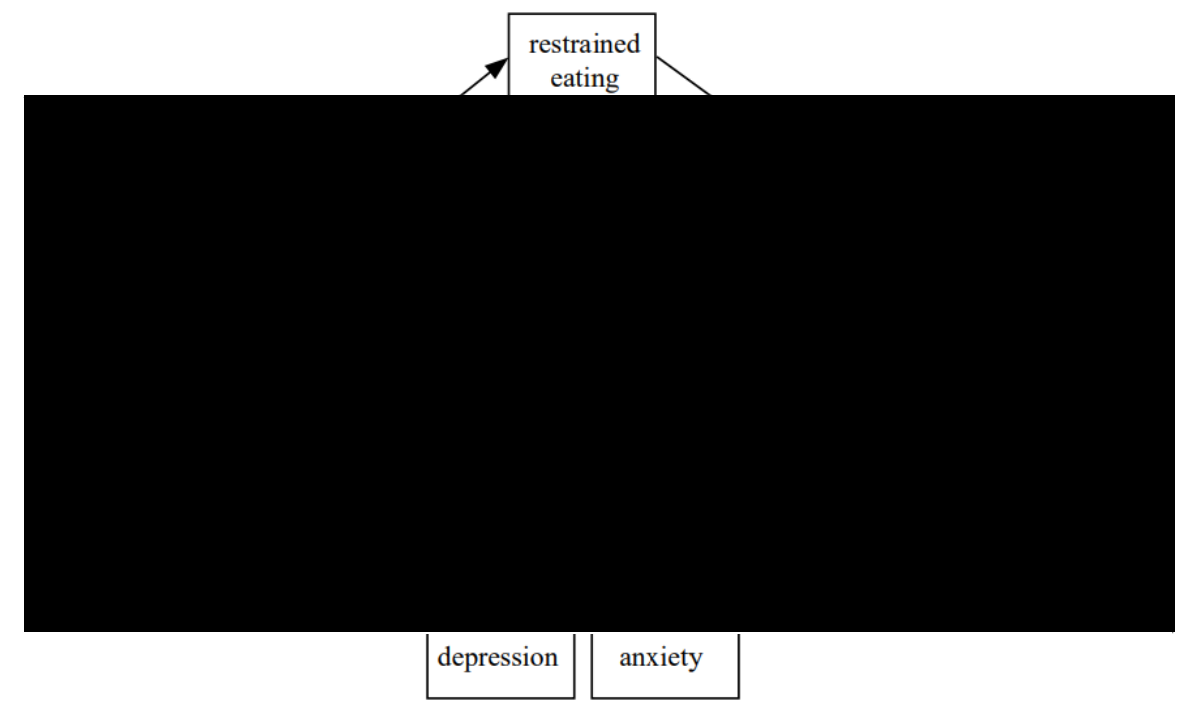

Extended dual pathway model of overeating by van Strien et al. (2005). Taken from van Strien et al. (2005).

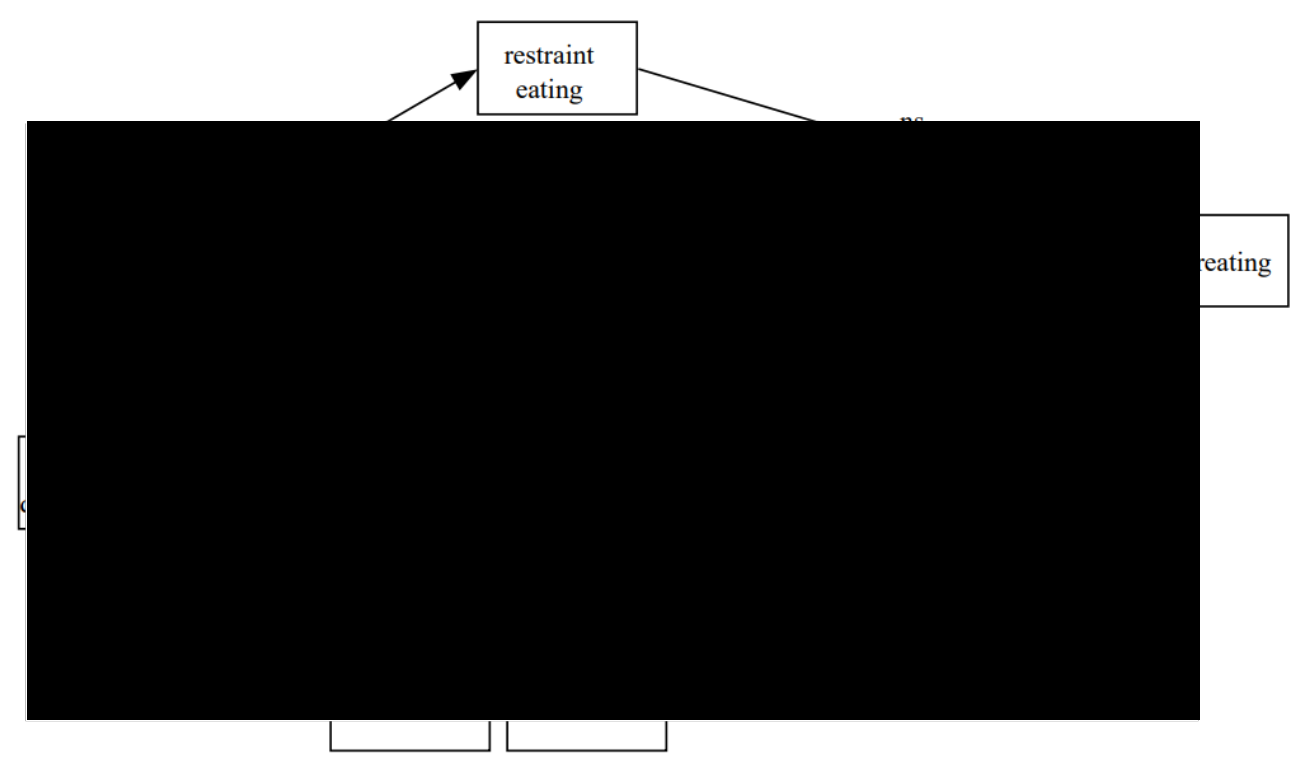


Appendix Y. Study Five: Ethics Memo

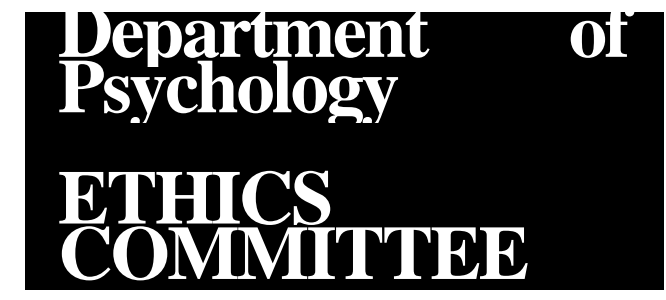

\section{Memo}

To:

Dr. Menna Price

Ms. Sophie Harrison

(using previously approved application submitted by

Amy Davies, Aimee Pink, Menna Price, Michelle Lee)

From: Dr. Jeremy Tree

Departmental Ethics Committee

Date: $\quad 6^{\text {th }}$ November 2017

Re: Investigation into the relationship between Alexithymia and perfectionism and body image

Your proposed study, "Investigation into the relationship between Alexithymia and perfectionism and body image", has been reviewed and is approved. Provided that the information obtained is kept absolutely confidential and that no personally identifiable information is entered on computer, you may proceed with your studies.

In order for your study to be displayed on the Experiment Management System (Participant Pool):

Forward this approval via email to Dr. Phil Tucker

\section{$\underline{\text { AND }}$}

Send a request for your study to be made visible, via the link on the EMS website (see Researcher Documentation for details). 


\section{Appendix Z. The Multidimensional Assessment of Interoceptive}

\section{Awareness (MAIA)}

Mehling et al. (2012)

\section{Noticing}

1. When I am tense I notice where the tension is located in my body.

2. I notice when I am uncomfortable in my body.

3. I notice where in my body I am comfortable.

4. I notice changes in my breathing such as whether it slows down or speeds up

28. When I am upset, I take time to explore how my body feels.

29. I listen to my body to inform me about what to do.

\section{Trusting}

30. I am at home in my body.

31. I feel my body is a safe place.

32. I trust my body sensations. 


\title{
Appendix AA. The Dutch Eating Behaviour Questionnaire
}

\author{
van Strien et al. (1986)
}

R 1. If you have put on weight, do you eat less than you usually do?*

2. Do you try to eat less at mealtimes than you would like to eat?

3. How often do you refuse food or drink offered because you are concerned about your weight?
27. If you have something delicious to eat, do you eat it straight away?

28. If you walk past the baker do you have the desire to buy something delicious?

29. If you walk past a snackbar or a cafe, do you have the desire to buy something delicious?

30. If you see others eating, do you also have

23. Do you have a desire to eat when you are bored or restless?*

Ext. 24. If food tastes good to you, do you eat more than usual?

25. If food smells and looks good, do you eat more than usual?

26. If you see or smell something delicious, do you have a desire to eat it? 


\title{
Appendix AB. The Body Attitudes Questionnaire
}

\author{
Ben-Tovim \& Walker (1991)
}

The following statements relate to attitudes and perceptions of your body image. Please read each one and select the response that shows how much you agree or disagree with the statement.

Strongly

Strongly

I feel when I can no longer get into clothes that used to fit me.

I try to avoid clothes which make me especially aware of my shape. 
Appendix AC. Study Five: Visual Analogue Scales

Please use the slider to indicate how attractive you feel right now:

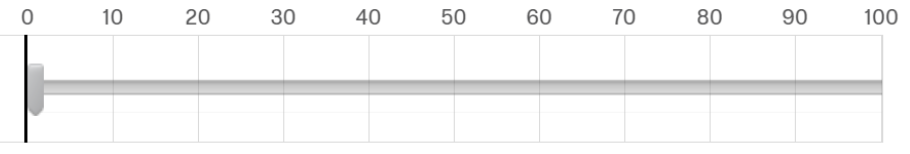




\section{Appendix AD. Study Five: Feeling Fat Manipulation Instructions}

The next part of this study relates to reactions to social situations. You will be presented with a short vignette. Please take a few minutes to read the scenario and try to imagine yourself as experiencing the scenario. Write in the text box a few sentences on how you might feel or what you might think in this situation.

Negative Feeling Fat: You are eating out with a group of close friends. You decide to order a burger and fries. You are very hungry and looking forward to a nice meal. When the food arrives your friend makes a comment, "that's really unhealthy, should you really be eating such fatty foods?" You look around and feel everyone's eyes on you. You notice they have ordered salads. Looking around the table you realise you are the biggest person there and your clothes look much tighter. You go bright red and feel embarrassed.

Neutral: You are eating out with a group of close friends. You decide to order a burger and fries. You are very hungry and looking forward to a nice meal. When the food arrives your friend makes a comment, "that looks so tasty, I can't wait for mine!" You look around and you notice your friends all ordered similar meals. The restaurant was quite pleasant and warm, and the décor was colourful. The staff members were polite and very helpful. You all dig in and you feel satisfied with the meal.

Positive Feeling Fat: You are eating out with a group of close friends. You decide to order a burger and fries. You are very hungry and looking forward to a nice meal. When the food arrives your friend makes a comment, "you are so lucky you can eat fatty foods but still look amazing!" You look around and everyone is nodding in agreement with her. You feel confident in yourself and happily enjoy the meal with your friends. You can't help but notice how well your clothes fit compared to some of your other friends. 
Negative General: You go to meet your friends at the pub. You greet them but no one acknowledges you. You say hello again and two friends turn to look at you, and give you an unfriendly look. They go to turn their back on you, whispering something to each other and laughing. You approach them and ask them why they won't speak to you. They whisper something to each other. They get the attention of the rest of the group and tell them it's time to leave. They leave you without explaining why they won't speak to you. 